\title{
The kinase MK2 in DNA replication upon genotoxic stress and chemotherapy
}

\author{
PhD Thesis \\ in partial fulfilment of the requirements \\ for the degree "Doctor of Natural Sciences (Dr. rer. nat.)" \\ in the Molecular Biology Program \\ at the Georg August University Göttingen, \\ Faculty of Biology
}

submitted by

Frederik Köpper

born in

Ostercappeln, Germany 
PhD Thesis committee:

Prof. Dr. Matthias Dobbelstein, Faculty of Medicine, University of Göttingen Prof. Dr. Jürgen Wienands, Faculty of Medicine, University of Göttingen Prof. Dr. Michael Kessel, Max Planck Institute for Biophysical Chemistry, Göttingen

Date of oral exam: October 17, 2012 


\section{AFFIDAVIT}

Herewith I declare, that I prepared the PhD Thesis: "The kinase MK2 in DNA replication upon genotoxic stress and chemotherapy" on my own and with no other sources and aids than quoted.

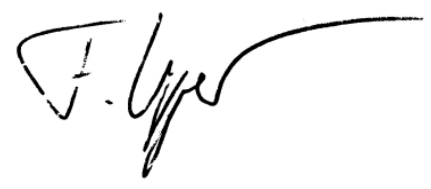

Göttingen, 11.09.2012 


\section{ACKNOWLEDGMENTS}

I would not have been able to carry out this project on my own. I am indebted...

First of all, to my supervisor, Prof. Matthias Dobbelstein. For giving me the opportunity to do my PhD in his lab. For introducing me to the fascinating field of cancer research, for his guidance, advice and support, and for never running out of ideas.

To my thesis committee, Prof. Michael Kessel and Prof. Jürgen Wienands, for fruitful discussions, constructive criticism and input.

To Kerstin Grüniger, Dr. Steffen Burkhardt and Ivana Jurik from the Molecular Biology Program coordination office for all their organization and help, especially during the first weeks and months of my PhD.

To those who provided financial support: The Molecular Biology Program, the Göttingen Graduate School for Neurosciences, Biophysics, and Molecular Biosciences (GGNB), the Studienstiftung des deutschen Volkes, and the European Cooperation in Science and Technology (COST).

To those who contributed to this work: Prof. Matthias Gaestel for providing the mice, Dr. Margarete Schön and Prof. Michael Schön for irradiating them, Ingegerd Elvers for introducing me to the art of DNA fiber spreads, Priyanka Saini for sharing data.

To Cathrin Bierwirth. I thank you for also sharing data, but much more for sharing the highs and lows of the project with me, for the great collaboration and technical support. I enjoyed working with you!

To all members, present and former, of the Department of Molecular Oncology. Four years of PhD can be tough, but it would have been much harder if it had not been for your endless help and readiness to share experience, thoughts, and to provide help whenever I needed it. I am very, very grateful that I could spend this time with you guys!

To Veena Jagannathan. For discussing results, for hypothesizing and for help in wrestling the fibers.

To Broder Schmidt, for sharing many a beer and many a thought during these years.

To Anna. I cannot thank you enough for all your help, your patience and energy.

Last, not least, to my parents. Your support, encouragement and faith mean everything to me. 


\section{TABLE OF CONTENTS}

TABLE OF CONTENTS

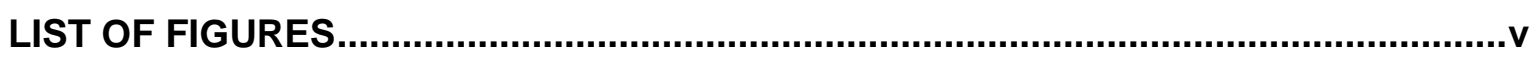

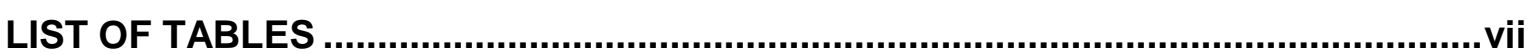

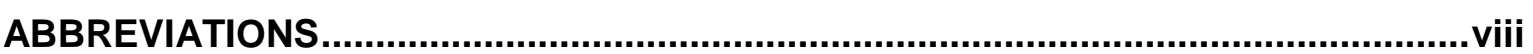

I ABSTRACT

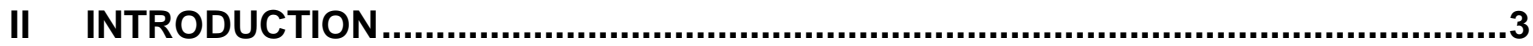

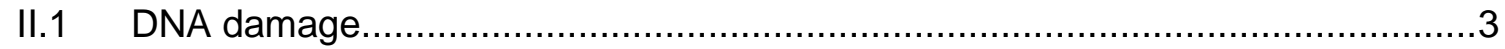

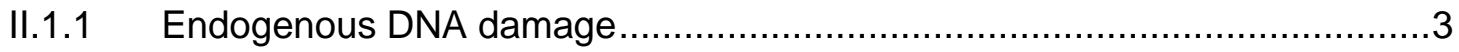

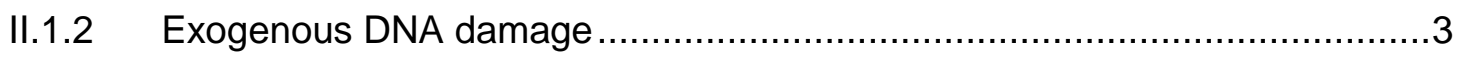

II.1.2.1 Ultraviolet and ionizing irradiation.................................................

II.1.2.2 DNA modifiers and inhibitors of topoisomerases .................................

II.1.2.3 Antimetabolites and nucleoside analogs .........................................

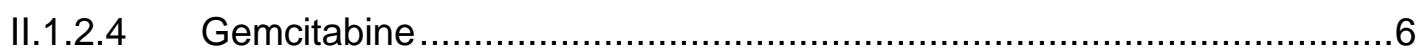

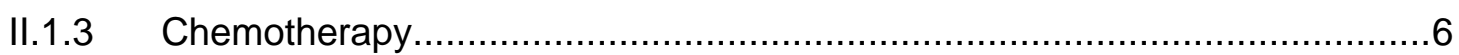

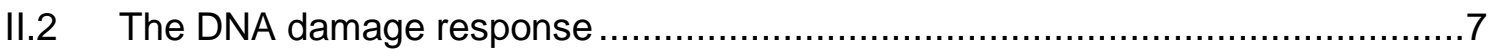

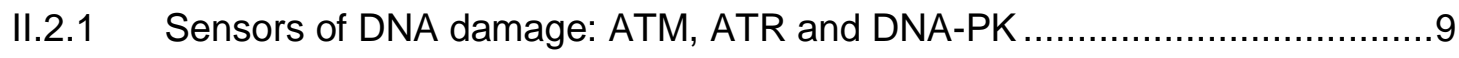

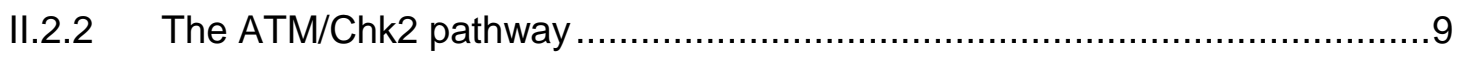

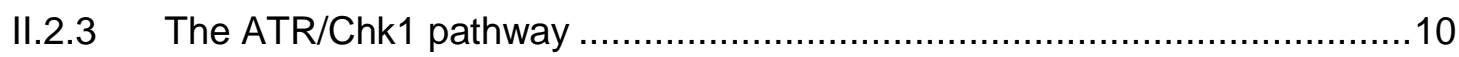

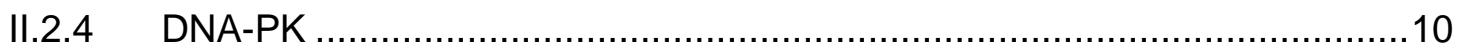

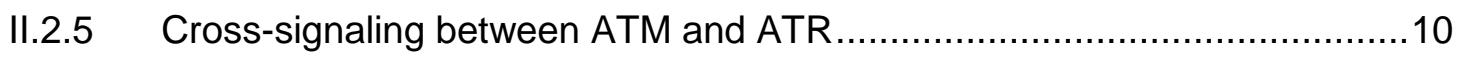

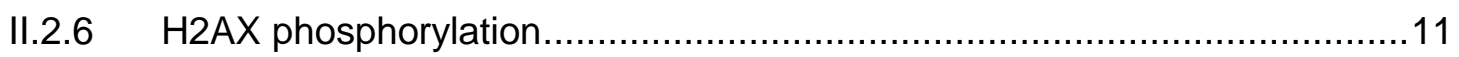

II.2.7 The cellular response to DNA damage ..................................................

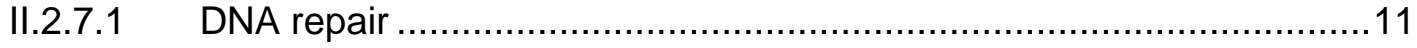

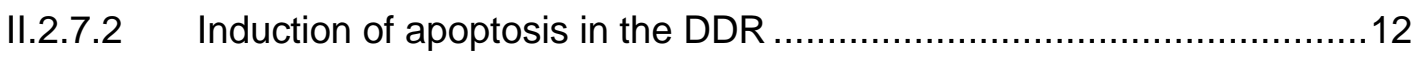

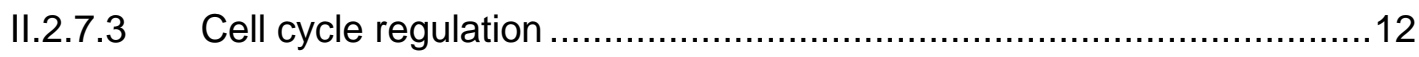

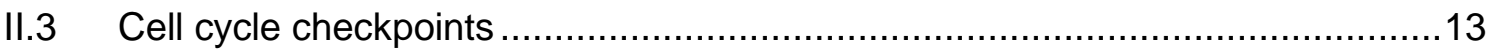

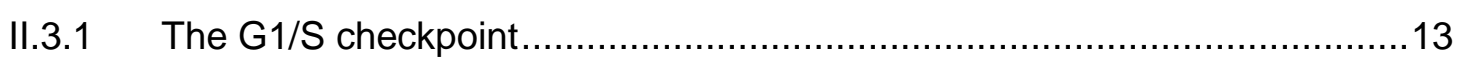

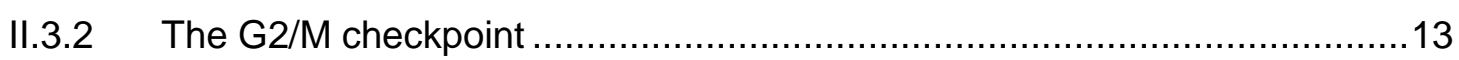

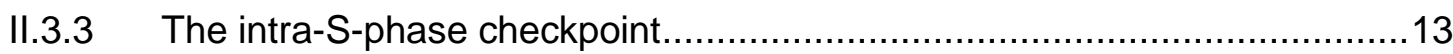

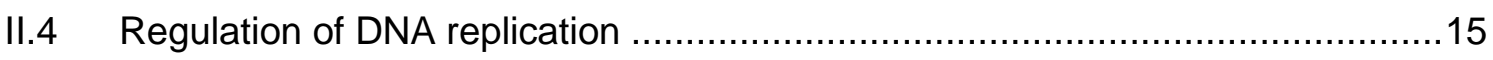

II.4.1 Origin firing during unperturbed S-phase.............................................15

II.4.2 Fork stabilization and origin firing under replicative stress.........................15 


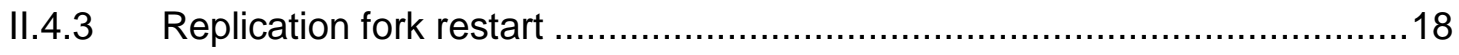

II.4.4 Translesion synthesis ............................................................... 18

II.4.4.1 Regulation of translesion synthesis ..............................................19

II.4.4.2 Global models for translesion synthesis ......................................20

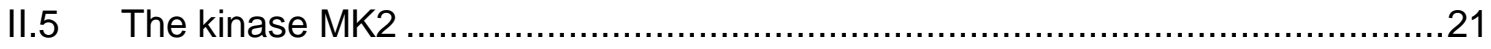

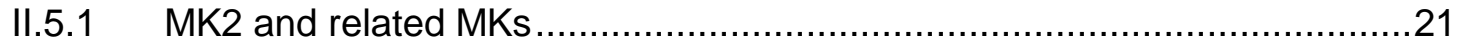

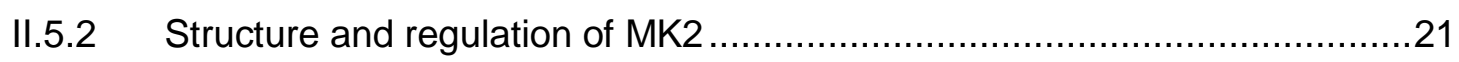

II.5.3 Substrates of MK2 and regulated processes .........................................22

II.5.4 MK2 in the DNA damage response ...............................................23

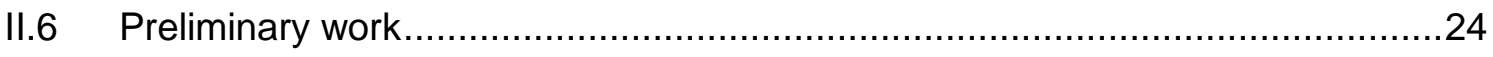

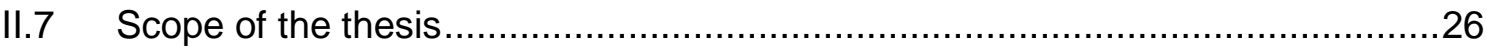

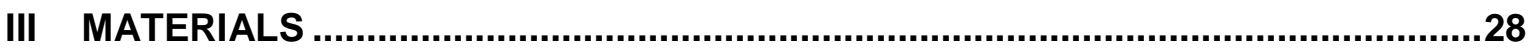

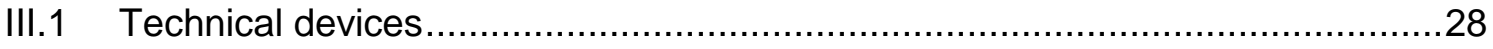

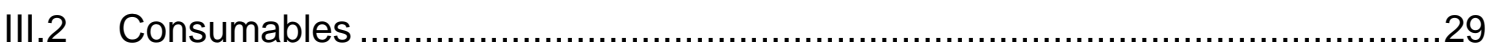

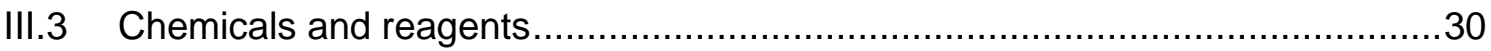

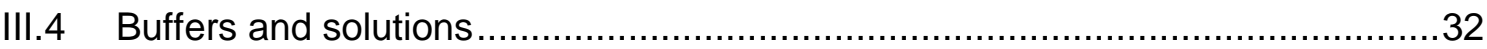

III.5 Chemotherapeutics and pharmacological inhibitors.........................................33

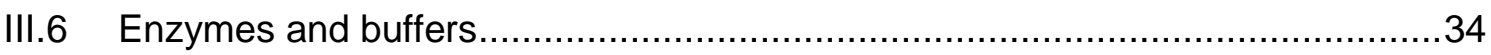

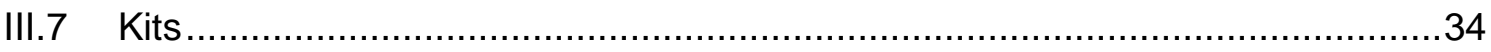

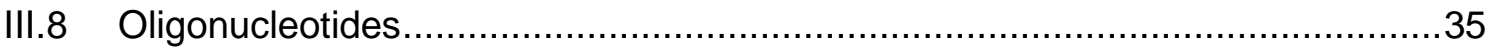

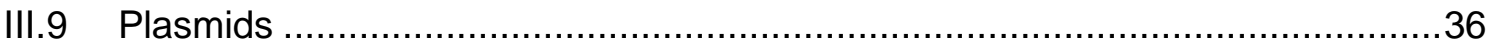

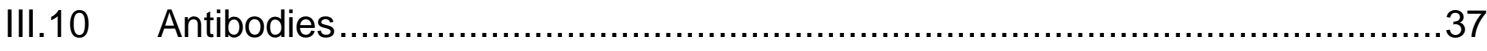

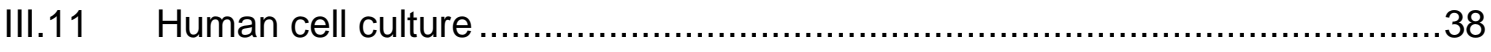

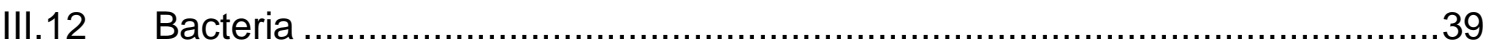

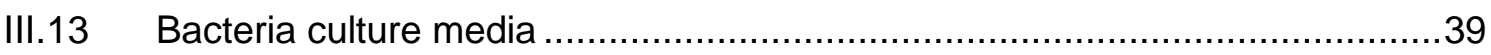

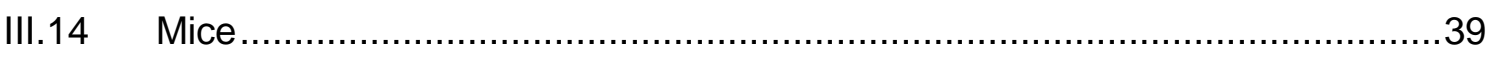

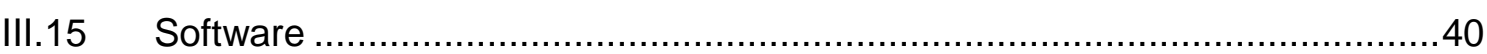

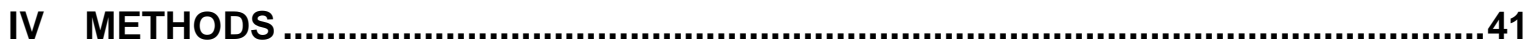

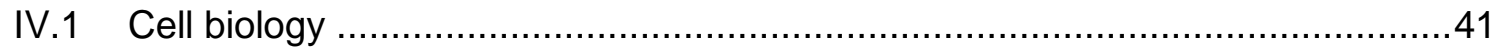

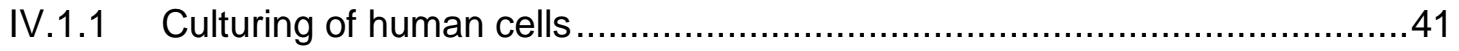

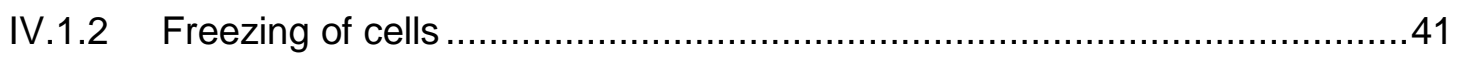

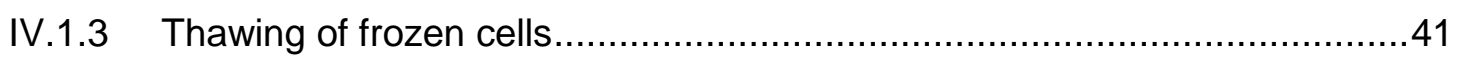

IV.1.4 Transfection of human cells................................................................ 42

IV.1.4.1 Transient transfection with siRNAs ............................................ 42

IV.1.4.2 Transient transfection with expression vectors ...............................42

IV.1.4.3 Generation of stably transfected U2OS cells ..................................43 


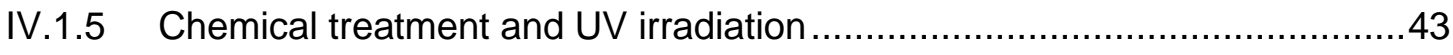

IV.1.6 Cell synchronization by thymidine block ..............................................4

IV.1.7 Generation of cell lysates for SDS-PAGE analysis ................................44

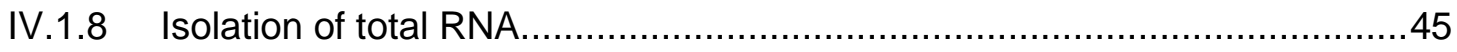

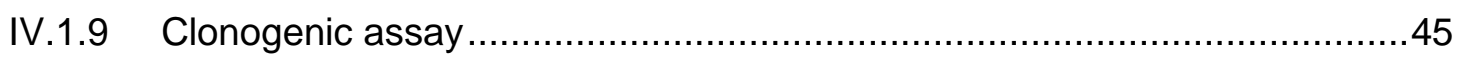

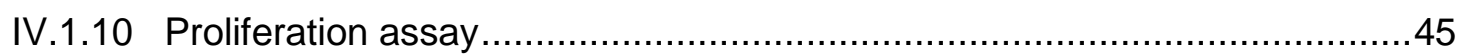

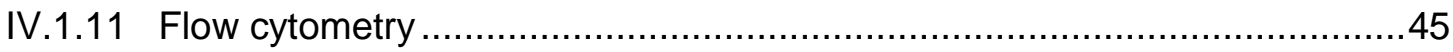

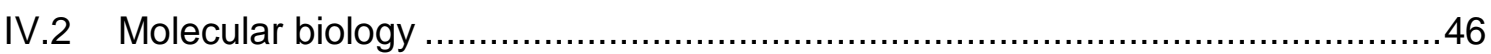

IV.2.1 Transformation of electro-competent bacteria .....................................46

IV.2.2 Isolation of plasmid DNA ....................................................................

IV.2.3 Determination of nucleic acid concentrations …...................................47

IV.2.4 Polymerase chain reaction ................................................................ 47

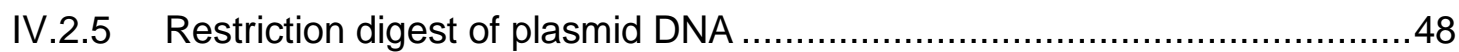

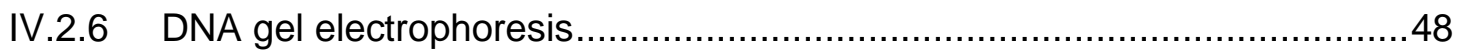

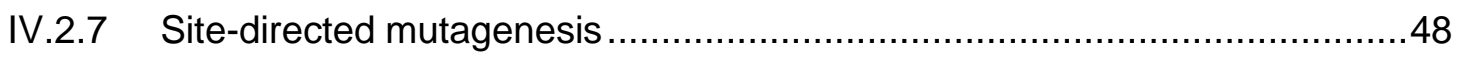

IV.2.8 Cloning of Myc-MK2 WT into pIRESneo ................................................ 49

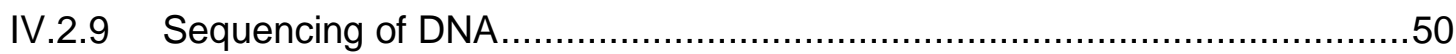

IV.2.10 Quantification of messenger RNA by PCR ........................................51

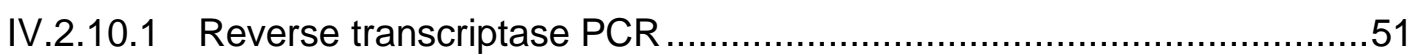

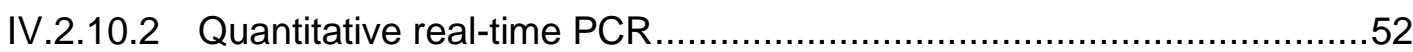

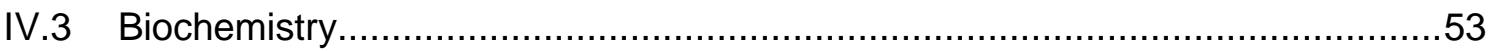

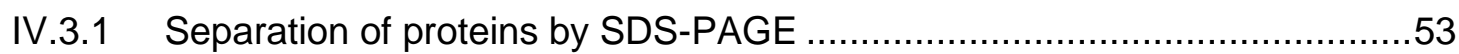

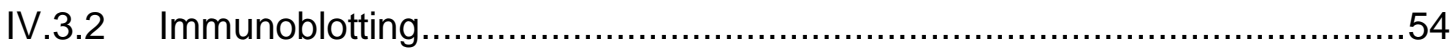

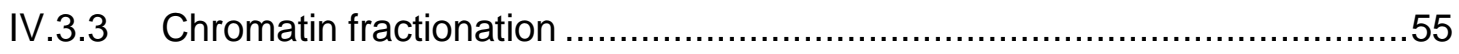

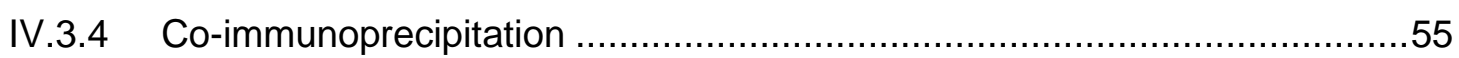

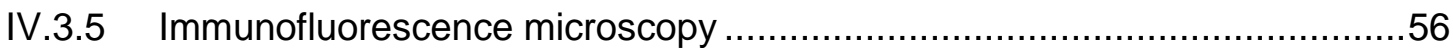

IV.4 High-content immunofluorescence microscopy ........................................57

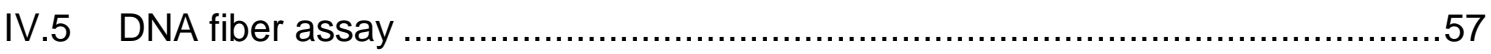

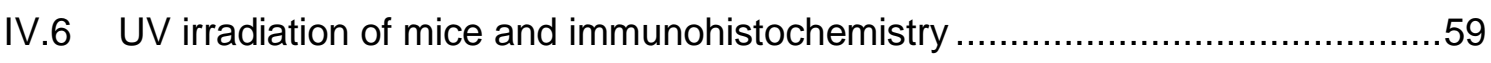

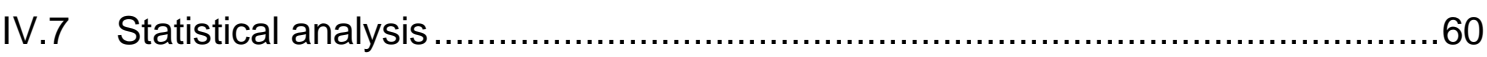

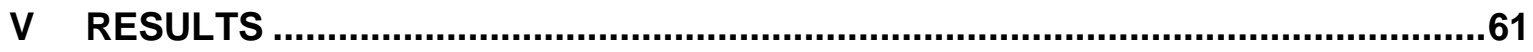

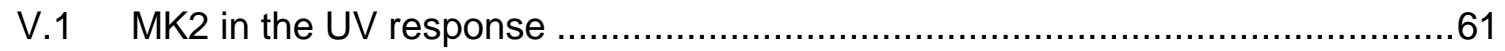

V.1.1 MK2 knockdown or pharmacological inhibition attenuates the DNA damage response upon UV irradiation in U2OS cells

V.1.2 UV-induced H2AX accumulation in keratinocytes depends on MK2 activity62

V.1.3 MK2 impairs cell survival upon UV irradiation in vitro and in vivo ...............63

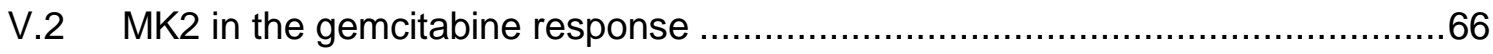

V.2.1 MK2 is a determinant of gemcitabine sensitivity .....................................66 
V.2.2 MK2 is required for the DDR in gemcitabine-sensitive pancreatic carcinoma cell lines.

V.2.3 MK2 acts during DNA replication................................................. 70

V.2.4 MK2 activity slows down DNA replication in response to gemcitabine while enhancing origin firing

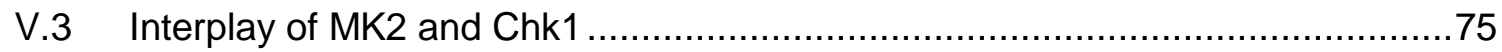

V.3.1 Reduction of Chk1 levels leads to an enhanced DNA damage response and cell cycle arrest, but these effects depend on MK2 …....................................... 75

V.3.2 Depletion of Wee1 induces genotoxic stress that is independent of MK2 ...77

V.3.3 Depletion of MK2 alleviates the replication block induced by Chk1 depletion and promotes cell survival

V.3.4 Chk1 inhibition attenuates replication fork progression while enhancing origin firing, and both effects depend on MK2 ................................................. 80

V.3.5 Several known regulators of origin firing are unaffected by MK2 ...............80

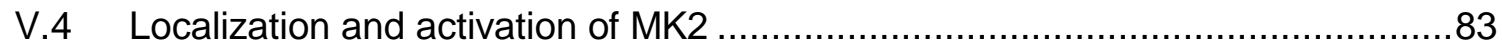

V.4.1 Upon genotoxic stress MK2 is not completely exported from the nucleus...83

V.4.2 p38 is required for Hsp27 phosphorylation but not for MK2-dependent DNA

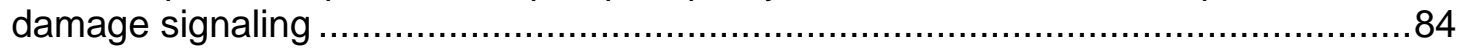

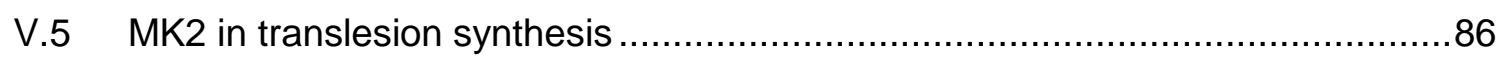

V.5.1 PCNA mono-ubiquitination induced by Chk1 depletion depends on MK2 ...86

V.5.2 Translesion synthesis is required for the rescue of gemcitabine-induced replication impairment by MK2 inhibition

VI DISCUSSION

VI.1 Tipping the balance: MK2 in the response to replicative stress ......................91

VI.2 Exploiting MK2 activity in chemotherapy ...............................................93

VI.3 MK2 in checkpoint signaling and apoptosis .............................................95

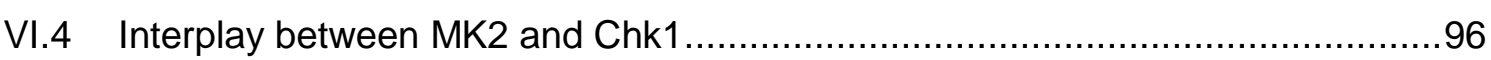

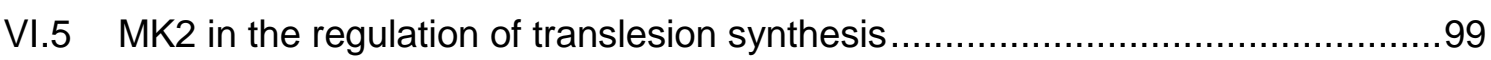

VI.6 Conclusions and future perspectives .................................................... 102

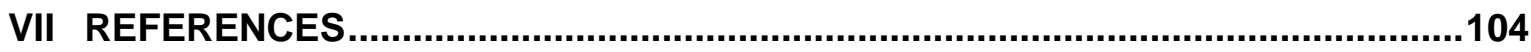

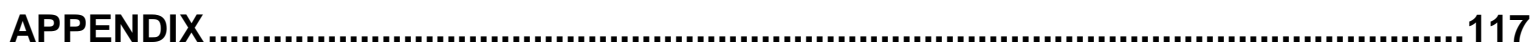

CURRICULUM VITAE..................................................... Fehler! Textmarke nicht definiert. 


\section{LIST OF FIGURES}

Figure II.1. DNA lesions induced by UV irradiation. .............................................. 4

Figure II.2. Structure of the nucleoside analog gemcitabine..........................................

Figure II.3. Kinase signaling in the DNA damage response. ..................................... 8

Figure II.4. Regulation of origin firing and fork stabilization upon replicative stress..........14

Figure II.5. A model of how origin clusters are regulated upon replicative stress. ............16

Figure II.6. Induction of translesion synthesis upon replication fork stalling. ...................19

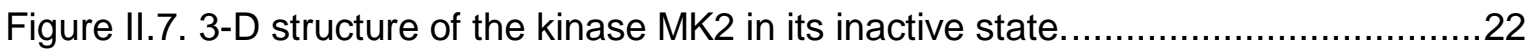

Figure II.8. RNAi kinase screening identifies kinases influencing H2AX phosphorylation

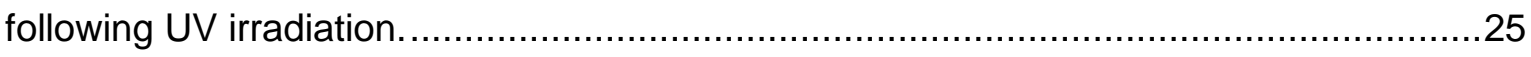

Figure IV.1. Representative images of DNA fibers obtained by DNA fiber assay and schematic representation of structures observed.

Figure V.1. Cells irradiated with UV display reduced levels of $\mathrm{YH} 2 \mathrm{AX}$ upon MK2 inhibition and depletion

Figure V.2. Accumulation and removal of CPD adducts upon UV irradiation is independent of MK2.

Figure V.3. MK2 inhibition and overexpression of a kinase-dead MK2 impair UV-induced H2AX phosphorylation in keratinocytes.

Figure V.4. MK2 depletion reduces UV-induced cell death.

Figure V.5. MK2/MK3 knockout-mice display reduced apoptosis in skin after UV

exposure.

Figure V.6. Gemcitabine-induced H2AX phosphorylation and stress signaling depends on MK2.

Figure V.7. Gemcitabine-induced cell death depends on MK2.

Figure V.8. Gemcitabine-induced H2AX phosphorylation is reduced by MK2 inhibition in gemcitabine-sensitive pancreatic carcinoma cell lines.

Figure V.9. Gemcitabine-induced cell death is reduced by MK2 inhibition in gemcitabinesensitive pancreatic carcinoma cell lines.

Figure V.10. MK2 inhibition does not affect cell cycle progression but acts during DNA replication upon DNA damage.

Figure V.11. Reduction of replication fork speed caused by gemcitabine is rescued by MK2 inhibition. 
Figure V.12. Increase of origin firing caused by gemcitabine is rescued by MK2 inhibition.

Figure V.13. H2AX phosphorylation upon Chk1 depletion or inhibition depends on MK2.76 Figure V.14. H2AX phosphorylation upon Wee1 depletion does not depend on MK2 .....77

Figure V.15. The replication block induced by Chk1 depletion depends on MK2 ............78

Figure V.16. Cell proliferation after Chk1 depletion is improved by co-depletion of MK2..79

Figure V.17. MK2 does not physically interact with Chk1. .79

Figure V.18. Reduced replication fork speed and increased origin firing upon Chk1 inhibition depend on MK2

Figure V.19. Depletion of MK2 and Chk1 does not affect several regulators of origin firing.

Figure V.20. Upon genotoxic stress, MK2 is not completely exported from the nucleus...84 Figure V.21. Inhibition of MK2 but not of p38 impairs gemcitabine-induced H2AX and JNK phosphorylation. .85

Figure V.22. MK2 mutated at a putative ATM/ATR phosphorylation site impairs gemcitabine-induced $\mathrm{H} 2 \mathrm{AX}$ phosphorylation. .85

Figure V.23. PCNA mono-ubiquitination induced by Chk1 depletion depends on MK2 ...87 Figure V.24. Rescue of gemcitabine-induced slow replication fork speed by MK2 inhibition depends on translesion synthesis. .89

Figure VII.1. A model of how MK2 functions in replicative stress. 92 


\section{LIST OF TABLES}

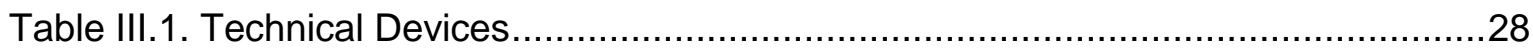

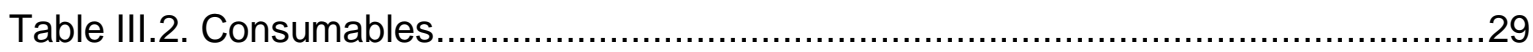

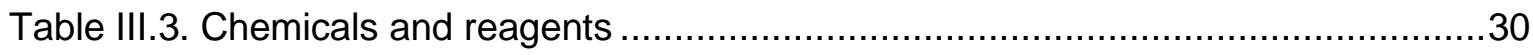

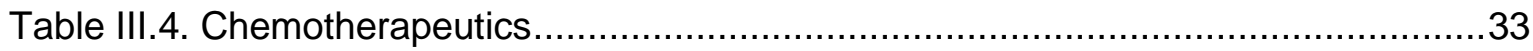

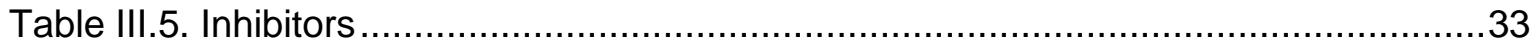

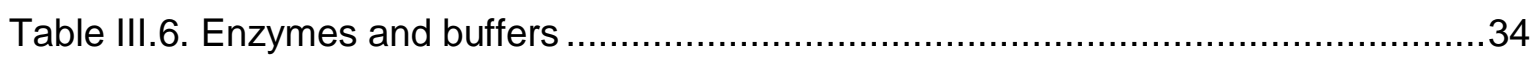

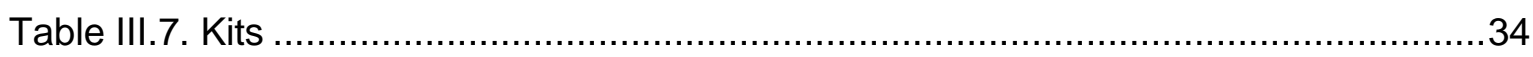

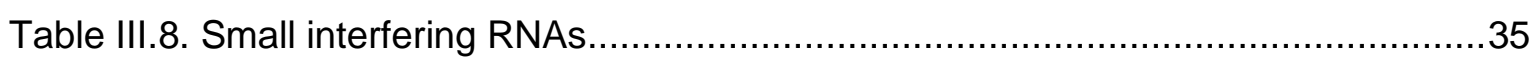

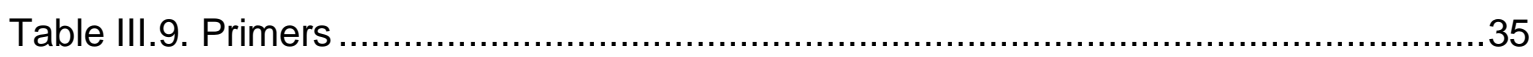

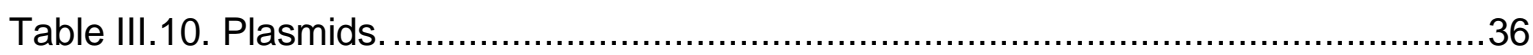

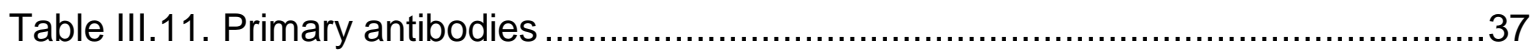

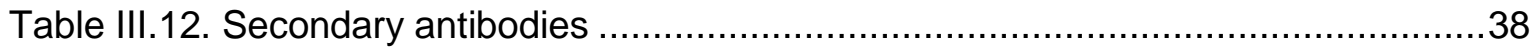

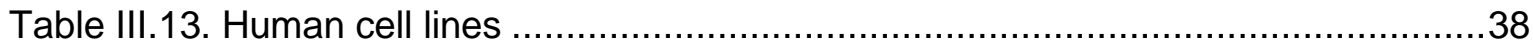

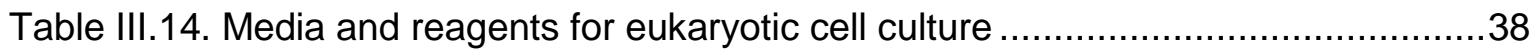

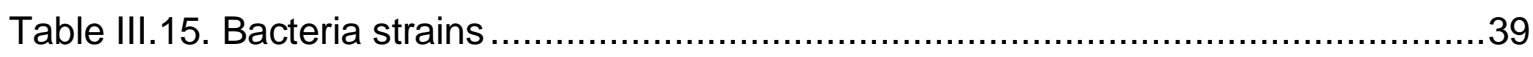

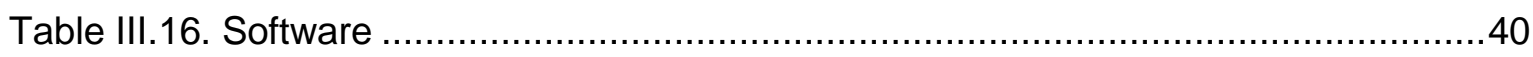

Table IV.1. Culture media for human cell lines..........................................................41

Table IV.2. Concentrations of chemicals used for cell treatment.....................................43

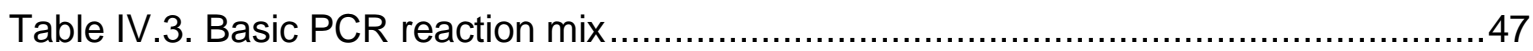

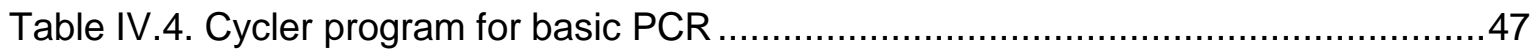

Table IV.5. PCR reaction mix for site-directed mutagenesis ........................................49

Table IV.6. Cycler program for site-directed mutagenesis ............................................49

Table IV.7. Ligation of Myc-MK2 WT into pIRESneo .................................................50

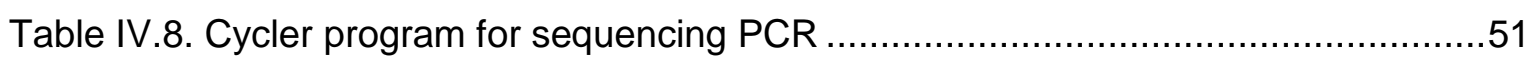

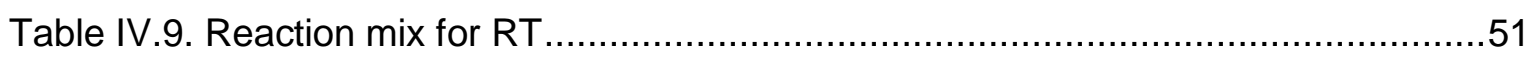

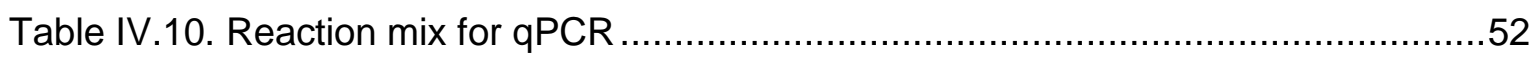

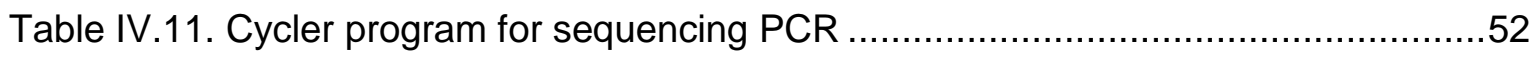

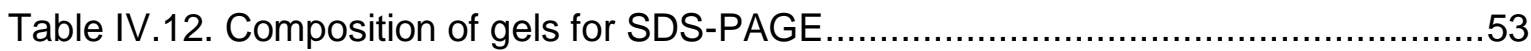




\section{ABBREVIATIONS}

\begin{tabular}{|c|c|}
\hline${ }^{\circ} \mathrm{C}$ & Degree Celcius \\
\hline$\mu g$ & Microgram \\
\hline$\mu L$ & Microliter \\
\hline$\mu \mathrm{M}$ & Micromolar \\
\hline 5-FU & 5-Fluorouracil \\
\hline $9-1-1$ & Rad9-Hus1-Rad1 \\
\hline$A$ & Adenine \\
\hline APS & Ammonium persulfate \\
\hline ATM & Ataxia telangiectasia mutated \\
\hline ATP & Adenosine triphosphoate \\
\hline ATR & ATM- and Rad3-related \\
\hline ATRIP & ATR interacting protein \\
\hline $\mathrm{bp}$ & Base pair \\
\hline BrdU & Bromodeoxyuridine \\
\hline BSA & Bovine serum albumine \\
\hline CDK & Cyclin-dependent kinase \\
\hline cDNA & Complementary DNA \\
\hline Chk1 & Checkpoint kinase 1 \\
\hline Chk2 & Checkpoint kinase 2 \\
\hline CldU & Chlorodeoxyuridine \\
\hline $\mathrm{cm}$ & Centimeter \\
\hline ColP & Co-immunoprecipitation \\
\hline CPD & Cyclobutane pyrimidine dimer \\
\hline C-terminus & Carboxy terminus \\
\hline ddNTP & Dideoxynucleotide triphosphate \\
\hline DDR & DNA damage response \\
\hline DMSO & Dimethylsulfoxide \\
\hline DNA & Deoxyribonucleic acid \\
\hline DNA-PK & DNA-dependent protein kinase \\
\hline dNTP & Deoxynucleotide triphosphate \\
\hline DSB & Double strand break \\
\hline DTT & Dithiotreitol \\
\hline dUTP & Deoxyuridine triphosphate \\
\hline EDTA & Ethylene diamine tetraacetic acid \\
\hline EdU & 5-Ethynyl-2'-deoxyuridine \\
\hline EGTA & Ethylene glycol tetraacetic acid \\
\hline ERK & Extracellular signal-regulated kinase \\
\hline $\mathrm{EtBr}$ & Ethidium bromide \\
\hline $\mathrm{EtOH}$ & Ethanol \\
\hline FCS & Fetal calf serum \\
\hline G & Gauge \\
\hline g & Gravitational force \\
\hline $\mathrm{h}$ & Hour \\
\hline $\mathrm{H} 2 \mathrm{AX}$ & Histone variant $2 \mathrm{AX}$ \\
\hline $\mathrm{HR}$ & Homologous recombination \\
\hline HRP & Horseradish peroxidase \\
\hline Hsp27 & Heat shock protein of $27 \mathrm{kDa}$ \\
\hline & Immunofluorescence \\
\hline
\end{tabular}




\begin{tabular}{|c|c|}
\hline Inh & Inhibitor \\
\hline IRES & Internal ribosomal entry site \\
\hline JNK & C-Jun N-terminal kinase \\
\hline KD & Kinase dead \\
\hline $\mathrm{kDa}$ & Kilodalton \\
\hline KIU & Kallikrein inactivator unit \\
\hline M & Molar \\
\hline MAPK & Mitogen activated protein kinase \\
\hline MCM & Minichromosome maintenance \\
\hline MCS & Multiple cloning site \\
\hline MetOH & Methanol \\
\hline $\mathrm{mg}$ & Milligram \\
\hline $\min$ & Minute \\
\hline MK / MAPKAPK & MAPK activated protein kinase \\
\hline MK2/MK3 DKO & MK2/MK3 double knock-out \\
\hline $\mathrm{mL}$ & Milliliter \\
\hline $\mathrm{mM}$ & Millimolar \\
\hline mRNA & Messenger RNA \\
\hline $\mathrm{n}$ & Sample size \\
\hline NES & Nuclear export signal \\
\hline $\mathrm{ng}$ & Nanogram \\
\hline NHEJ & Non-homologous end joining \\
\hline $\mathrm{nM}$ & Nanomolar \\
\hline $\mathrm{nm}$ & Nanometer \\
\hline NP-40 & Nonidet $\mathrm{P}-40$ substitute \\
\hline n.s. & Not significant \\
\hline $0 / n$ & Over night \\
\hline $\mathrm{p}$ & Phospho \\
\hline PAS & Protein A sepharose \\
\hline PBS & Phosphate buffered saline \\
\hline PCNA & Proliferating cell nuclear antigen \\
\hline PCNAub1 & PCNA mono-ubiquitinated at K164 \\
\hline PCR & Polymerase chain reaction \\
\hline PI & Propidium iodide \\
\hline PIKK & Phosphatidylinositol 3-kinase related kinase \\
\hline PLK1 & Polo-like kinase 1 \\
\hline PRC1 & Polycomb repressive complex 1 \\
\hline pre-RC & Pre-replication complex \\
\hline qPCR & Quantitative real-time PCR \\
\hline RNA & Ribonucleic acid \\
\hline RNAi & RNA interference \\
\hline RNR & Ribonucleotide reductase \\
\hline ROS & Reactive oxygen species \\
\hline RPA & Replication protein A \\
\hline rpm & Rounds per minute \\
\hline RT & Room temperature; Reverse transcriptase \\
\hline SDS & Sodium dodecyl sulfate \\
\hline SDS-PAGE & SDS-polyacrylamide gel electrophoresis \\
\hline sec & Second \\
\hline siRNA & Small interfering ribonucleic acid \\
\hline ssDNA & Single-stranded DNA \\
\hline T & Thymine \\
\hline TAO & Thousand-and-one amino acid \\
\hline TBST & Tris buffered saline + Tween 20 \\
\hline TdT & Terminal deoxynucleotidyltransferase \\
\hline TEMED & Tetramethylethylenediamine \\
\hline
\end{tabular}




$\begin{array}{ll}\text { TLS } & \text { Translesion synthesis } \\ \text { Tris } & \text { Trisamine } \\ \text { TUNEL } & \text { TdT UTP nick end labeling } \\ \text { U } & \text { Unit } \\ \text { UV } & \text { Ultraviolet } \\ \text { UV-B } & \text { Ultraviolet light, wavelength } 302 \mathrm{~nm} \\ \text { UV-C } & \text { Ultraviolet light, wavelength } 254 \mathrm{~nm} \\ \text { V } & \text { Volt } \\ \text { WT } & \text { Wild type } \\ \text { YH2AX } & \text { H2AX phosphorylated on S319 } \\ \Phi & \text { Hydrophobic amino acid }\end{array}$

For amino acids, the single-letter code is used. 


\section{ABSTRACT}

DNA damage constitutes a constant threat to genomic integrity. Cells evolved programs controlled by a complex signaling network to cope with these lesions in order to avoid tumorigenesis. These cellular processes and the signaling cascades that regulate them form the DNA damage response (DDR). Whereas many aspects of the DDR have been investigated in great detail, comparably little is known about how cells respond to genotoxic stress during DNA replication.

Here, we identify a hitherto unknown function for the kinase MK2 in the control of replication upon genotoxic stress in S-phase. Originally described as a mediator of general stress signaling in the p38/MK2 pathway, recent studies reported a role of MK2 in checkpoint signaling. In our lab MK2 was previously found to be required for efficient phosphorylation of the histone variant $\mathrm{H} 2 \mathrm{AX}$ (yielding $\mathrm{YH} 2 \mathrm{AX}$ ), a hallmark of the DDR, upon DNA damage induced by ultraviolet (UV) irradiation. This suggests a more general function of the kinase in the DDR than anticipated.

We now report that depletion or inhibition of MK2 protects cells from the consequences of UV-induced DNA damage, and mice with genetic ablation of MK2 and its relative MK3 display strongly reduced apoptosis in the skin after UV irradiation. As UV-induced DNA damage mainly affects cells during replication but also in other phases of the cell cycle, we tested whether DNA damage induced by the S-phase-specific drug gemcitabine elicited an MK2-dependent DDR, as well. We found that inhibition or depletion of MK2 indeed reduces the accumulation of $\mathrm{yH} 2 \mathrm{AX}$ and increases cell viability following gemcitabine treatment, and this effect cannot be attributed to cell cycle modulation by MK2. MK2 inhibition also rescues slow replication fork progression and increased origin firing caused by gemcitabine, demonstrating that the kinase affects replication in response to DNA damage in S-phase.

We furthermore observed that MK2 is required for the genotoxic effects caused by inhibition or depletion of the essential checkpoint kinase Chk1 and that MK2 inhibition also alleviates deregulated replication caused by inhibition of Chk1. Such antagonistic activity between the two kinases comes as a surprise as both share the same target phosphorylation motif. We speculate that MK2 and Chk1 target different proteins, mediated by specific interaction partners not shared between the two.

In search for the mechanism underlying the effect of MK2 on replication, we did not find any influence on regulators of origin firing, arguing that MK2 acts directly at the replication fork. The rescue of gemcitabine-induced slow fork speed by MK2 inhibition suggests that 
the kinase controls lesion bypass mechanisms. Accordingly, we observed that the rescue of deregulated replication by MK2 inhibition depends on translesion synthesis (TLS). We speculate that MK2 directly targets components of the TLS machinery, thereby repressing TLS.

In conclusion, our data for the first time reveal an activity of MK2 in replication upon DNA damage. MK2 is required for slow fork speed and increased origin firing upon replicative stress, and this activity depends on TLS. We propose that MK2 balances the DDR by repressing TLS to limit the mutagenic effects of this lesion bypass mechanism, promoting DNA repair or cell death. These findings also identify the p38/MK2 pathway as a potential drug target as enhanced MK2 activation might sensitize cells to chemotherapy. 


\section{INTRODUCTION}

\section{II.1 DNA damage}

Every cell of the human body is constantly exposed to various kinds of genotoxic stress that challenges its genomic integrity. It has been estimated that no less than $10^{5}$ DNA lesions, most of them spontaneous, occur in each cell every day (Hoeijmakers, 2009; Lindahl, 2004).

However, cells rely on correct genetic information to ensure the functionality of all biological processes. Furthermore, the exact duplication of their genome is essential to pass on genetic information to the next generation. Any kind of damage to the DNA can result in cell death or, if mutations accumulate, enhanced proliferation and eventually to the development of cancer. Hence, genomic integrity is of vital importance to all cells and organisms.

There are several types of genotoxic stress that challenge genomic stability, and based on the source, one can distinguish between endogenous and exogenous DNA damage.

\section{II.1.1 Endogenous DNA damage}

Endogenous DNA damage is defined as any genotoxic stress that arises from biochemical processes from within the cell or organism. The most important sources of endogenous DNA damage are toxic metabolic byproducts such as reactive oxygen species (ROS). ROS oxidize components of the DNA. Also, misincorporated nucleotides during replication that are not always corrected for constitute a major source of endogenous DNA damage (De Bont and van Larebeke, 2004; Lindahl, 2004; Lindahl and Barnes, 2000). In cancer cells, levels of endogenous DNA damage are generally increased, mostly due to early mutations during tumorigenesis that affect the cell's capacity to cope with oxidative stress or DNA lesions (Jackson and Loeb, 2001).

\section{II.1.2 Exogenous DNA damage}

Additionally, cells are exposed to many different kinds of exogenous DNA damage, i.e. damage that results from sources outside the cell or organism. An overview of the most relevant kinds of exogenous DNA damage is given below. 


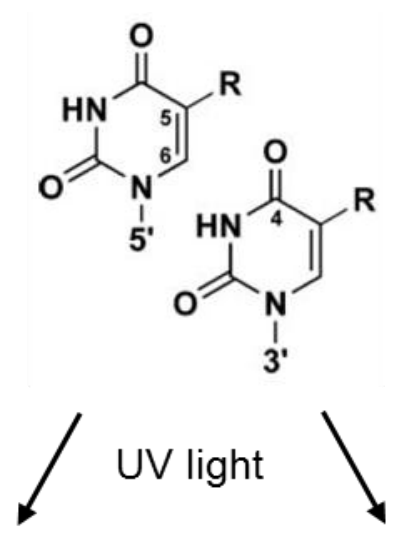

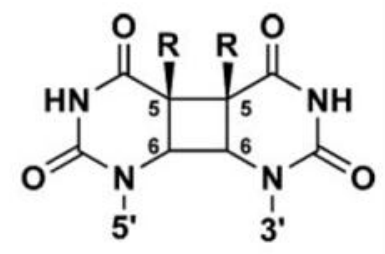

CPD

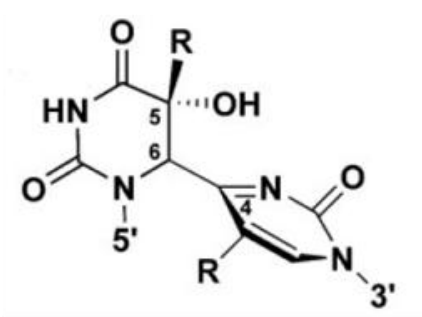

6-4 photoproduct

Figure II.1. DNA lesions induced by UV irradiation.

Absorption of energy from light of short wavelength $(240-400 \mathrm{~nm})$ by DNA results in the formation of 6-4 photoproducts and - more frequently - cyclobutane pyrimidine dimers. Figure from Li et al., 2006.

\section{II.1.2.1 Ultraviolet and ionizing irradiation}

The most frequent source of exogenous DNA damage is ultraviolet (UV) light originating from the sun. This radiation can be subdivided according to its wavelength to UV-A (320$400 \mathrm{~nm})$, UV-B (290-320 nm) and UV-C (240-290 nm). UV-C light does not usually penetrate the atmosphere so that life on earth is only naturally exposed to UV-A and UV$B$ light. The energy from UV irradiation is absorbed by DNA bases, inducing 6-4 photoproducts and cyclobutane pyrimidine dimers (CPDs) (see Figure II.1). These UVinduced lesions constitute an unbridgeable block to replicative DNA polymerases but are also toxic in other phases of the cell cycle. Importantly, UV irradiation also produces ROS and thus has a second mechanism of action to induce DNA damage and cellular stress (Herrlich et al., 2008).

lonizing radiation, on the other hand, is a comparably rare but potent inducer of DNA damage. Its high energy is absorbed by the DNA, resulting in double-strand breaks (DSBs) with often deleterious effects. 


\section{II.1.2.2 DNA modifiers and inhibitors of topoisomerases}

Another source of exogenous DNA damage is formed by chemicals that interact with or modify DNA. Many of the chemicals found in tobacco smoke potently induce aromatic DNA adducts. Alkylating agents like cyclophosphamide or the alkylating-like agent cisplatin as well as some antibiotics cause DNA inter- or intrastrand crosslinks. Furthermore, other antibiotics such as doxorubicin interfere with the function of topoisomerases, inducing DSBs. Topoisomerases are also blocked by toxins like irinotecan (Espinosa et al., 2003).

\section{II.1.2.3 Antimetabolites and nucleoside analogs}

Above-mentioned drugs are mostly non-selective in that they induce DNA damage irrespective of the cell cycle phase. Some DNA-damaging agents, however, only act on cells replicating their DNA in the S-phase of the cell cycle. This process is extremely sensitive to disturbance and any occurring DNA damage might result in mutations or a complete failure to replicate the DNA, leading to cell death. There are two kinds of agents that interfere with DNA replication which can be separated based on their mechanism of action: antimetabolites and nucleoside analogs (Espinosa et al., 2003).

Antimetabolites inhibit enzymes that are essential for the synthesis of deoxyribonucleotides (dNTPs), resulting in an imbalance of the cellular dNTP pools. This imbalance interferes with proper DNA replication. Hydroxyurea, for instance, inhibits the enzyme ribonucleotide reductase (RNR) while 5-fluorouracil (5-FU) blocks thymidilate synthase.

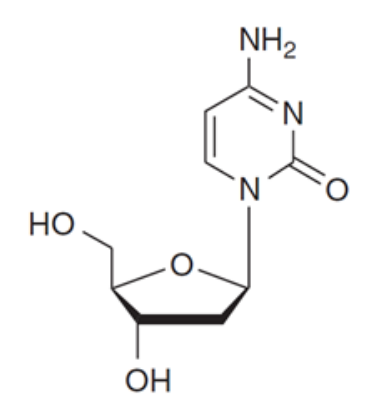

deoxycytidine

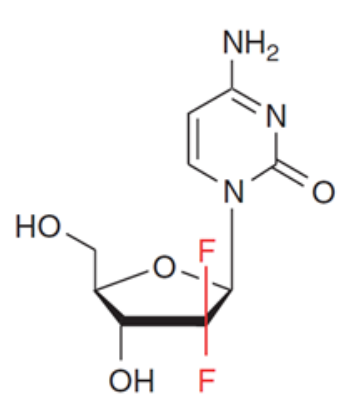

gemcitabine

Figure II.2. Structure of the nucleoside analog gemcitabine.

Gemcitabine is a deoxycitidine analog with two fluor atoms at the 2' carbon of the ribose. Figure from Ewald et al., 2008. 
Nucleoside analogs, on the other hand, are incorporated into DNA instead of the correct dNTPs. In most cases, DNA polymerases then fail to extend the synthesized strand beyond the incorporated analog and replication forks stall. If not removed by DNA repair mechanisms, mutations can arise from the misincorporated compounds (Espinosa et al., 2003; Galmarini et al., 2002). In this context, a special case is formed by the drug gemcitabine as it acts both as a nucleoside analog and an antimetabolite.

\section{II.1.2.4 Gemcitabine}

Gemcitabine (2',2'-difluorodeoxycytidine, also called dFdC) is a deoxycytidine analog with two fluor atoms at the 2' carbon of the ribose moiety (Figure II.2). Upon phosphorylation to its triphosphate, it is incorporated into DNA instead of deoxycytidine triphosphate. The replicative polymerase then attaches another nucleotide to the incorporated gemcitabine but fails to extend the strand further (Huang et al., 1991). It has been shown that this is due to a topological distortion of the DNA helix induced by gemcitabine; replicative polymerases cannot accommodate such a distorted helix in their active site (Konerding et al., 2002). The mechanism by which gemcitabine interferes with strand elongation is termed "masked termination" since elongation is not terminated immediately behind the incorporated gemcitabine (Plunkett, Anti-Cancer Drugs 1995). For this reason, gemcitabine cannot be removed by the polymerase's 3'-5' exonuclease activity (Huang et al., 1991). The DNA repair machinery also fails to identify and consequently to excise incorporated gemcitabine (Crul et al., 2003).

Gemcitabine furthermore acts as an antimetabolite inhibiting RNR. The resulting dNTP pool imbalance increases the probability of gemcitabine to be incorporated into DNA, enhancing its genotoxic potential, a mechanism known as self-potentiation (Ewald et al., 2008). However, its effect on strand elongation constitutes the major source of genotoxicity (Huang et al., 1991), especially when administered for short periods of time since inhibition of RNR only results in DNA damage after about $24 \mathrm{~h}$ (Petermann et al., 2010b).

\section{II.1.3 Chemotherapy}

Besides surgery and irradiation, chemotherapy is central to the treatment of cancer. Chemotherapy exploits a great variety of sources of DNA damage: Cancer cells are highly proliferative and therefore extremely sensitive to genomic insults, especially as cells from advanced cancers usually suffer from genomic instability due to mutations that affect their ability to respond to DNA damage (Bartek and Lukas, 2003). Many of above-mentioned DNA-damaging agents are used as anti-cancer drugs (Espinosa et al., 2003). Especially 
antimetabolites and nucleoside analogs are employed to specifically target cells in Sphase for two reasons: Firstly, cells in the process of replicating their DNA are highly sensitive to genotoxic stress. Secondly, due to their high proliferation rate, targeting Sphase is a potent means to kill cancer cells while reducing site effects on healthy, less proliferative tissue.

Importantly, each chemotherapeutic drug is usually only effective in a small range of tumor types. The reason for such narrow ranges of efficacy is still not completely understood (Ewald et al., 2008). Gemcitabine is used for the first-line treatment of pancreatic adenocarcinomas and in combination with other drugs in the treatment of e.g. advanced mammary and urothelial carcinoma as well as non-small cell lung cancer (Mini et al., 2006).

Although many chemotherapeutics have been in use for decades, little is known about the precise cellular response mechanism they trigger and the reasons underlying the often narrow spectrum of efficacy. Recent years saw increasing efforts to close this gap of knowledge as a better understanding of how chemotherapeutics act forms the basis for future improvements in cancer treatment.

\section{II.2 The DNA damage response}

Owing to the significance of genomic integrity, evolution has not left cells unprotected from genotoxic insults but equipped them with a range of mechanisms to cope with DNA damage. Genetic lesions are recognized by the cell and elicit signaling cascades that form a complex network and induce as well as regulate the cellular response. Depending on the kind and extent of DNA damage, cells can react very differently and activate a response that ranges from transcriptional changes over cell cycle arrest and DNA repair to the induction of apoptosis. The complete cellular program activated upon genomic insults, from the recognition of the damage to the regulation of cellular mechanisms to cope with it, is termed DNA damage response (DDR) (Zhou and Elledge, 2000).

Despite the constant discovery of new factors and pathways that contribute to the DDR over the last decades, we are still far from obtaining a complete picture of how cells protect their genome. Given the complexity of the DDR network, a brief overview will be provided here and only the proteins and pathways immediately relevant to the work presented will be introduced in detail.

Phosphorylation cascades constitute the core of the DDR. An overview is provided in Figure II.3. Whereas the regulation of the kinases catalyzing these phosphorylations in 
the DDR has been studied in great detail, only little is known about the contribution of phosphatases to DNA damage signaling (Medema and Macurek, 2012), but recent findings indicate that this contribution must not be underestimated (Freeman and Monteiro, 2010). One way to structure the proteins participating in the response is to dis-

\section{$X \times 1 \times$}

DSBs

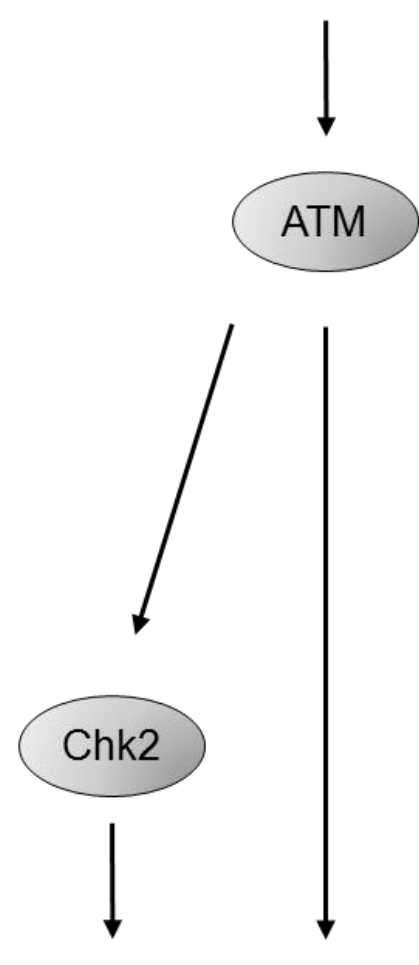

\section{س}

ssDNA

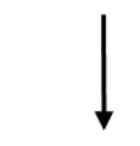

\section{ATR}
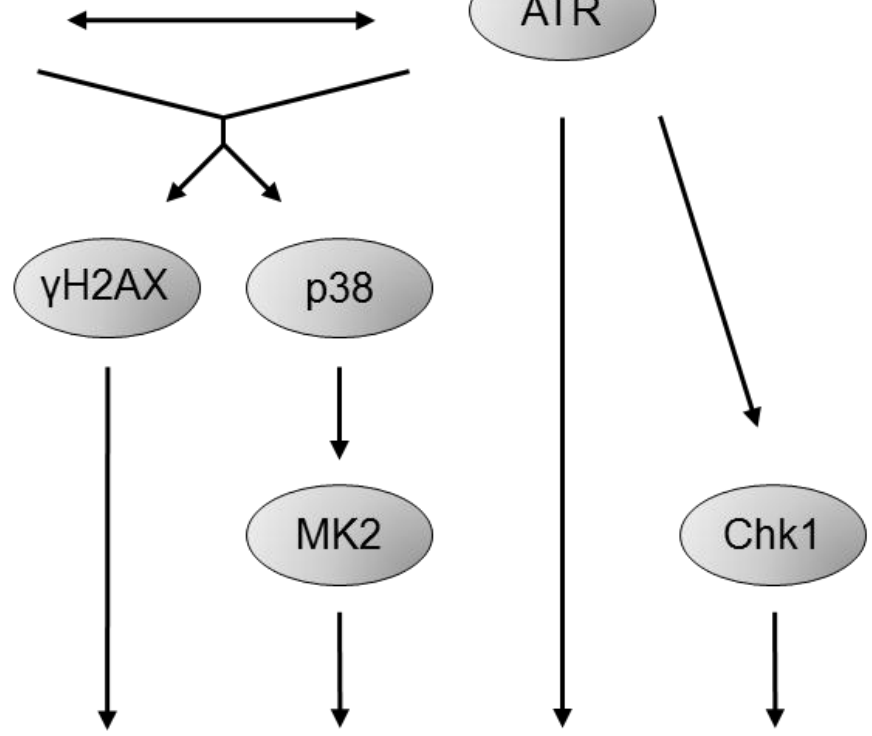

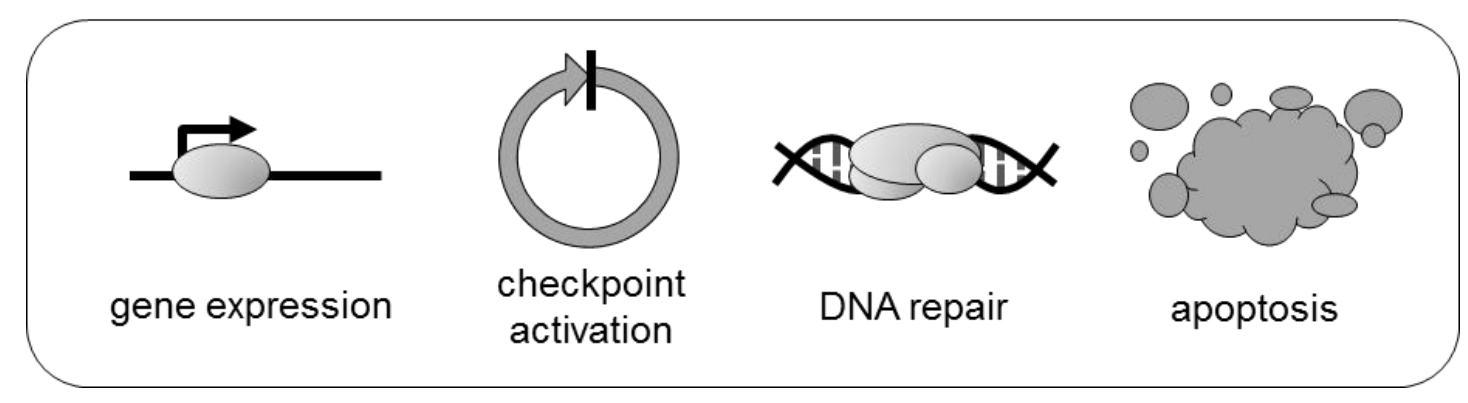

Figure II.3. Kinase signaling in the DNA damage response.

The sensor kinases ATM and ATR are activated by DSBs and ssDNA formed at stalled replication forks, respectively, and phosphorylate hundreds of target proteins, among them the mediators Chk1 and Chk2. Also, both ATM and ATR phosphorylate H2AX and induce the p38/MK2 pathway that contributes to checkpoint regulation. Depending on the kind and extent of the damage, DDR signaling results in regulation of gene expression, activation of cell cycle checkpoints, repair of DNA lesions or apoptosis. Both the ATM/Chk2 and the ATR/Chk1 pathway converge at several points and activate each other. 
tinguish between sensors, mediators and effectors (Zhou and Elledge, 2000). Sensors are involved in the immediate recognition of the damage and become activated at the site of the lesion. They pass on the damage signal to the mediators that in turn activate effector proteins that serve as regulators of the different response programs. While this differentiation is helpful, it is important to note that several proteins serve more than one function and could thus be classified as e.g. both sensor and mediator.

\section{II.2.1 Sensors of DNA damage: ATM, ATR and DNA-PK}

The most important sensors of DNA damage are members of the phosphatidylinositol 3kinase-related kinase (PIKK) family, namely ataxia-telangiectasia mutated (ATM), ATMand Rad3-related (ATR) and DNA-dependent protein kinase (DNA-PK). ATM, ATR and DNA-PK are serine/threonine (S/T) kinases that share the same minimal substrate phosphorylation motif, which is phospho-serine/threonine with glutamine in the +1 position (pS/T-Q). Upon DNA damage, these kinases phosphorylate hundreds of proteins (Matsuoka et al., 2007). Due to the shared substrate specificity, the majority of substrates can be phosphorylated by all three kinases. A subset of targets, however, is unique to each kinase. For this reason, ATM, ATR and - to a lesser extent - DNA-PK are capable of specifically activating separate response pathways. Generally, DNA damage results in the activation of all response pathways, but depending on the kind of damage, only one or few pathways are fully activated while the others serve back-up functions. Correspondingly, the DDR elicited critically depends on the kind of lesion, but all pathways result in the same pattern of cellular response programs (Sancar et al., 2004). Most kinds of genotoxic stress will ultimately lead to the formation of either DSBs or single-stranded DNA (ssDNA). ssDNA mainly arises in S-phase upon replicative stress: When encountering a lesion, the replicative polymerase stalls while the associated helicase continues to unwind the DNA, exposing long stretches of ssDNA (Feng et al., 2006).

\section{II.2.2 The ATM/Chk2 pathway}

DSBs robustly activate ATM. ATM's most important substrates are the transcription factor p53 and the checkpoint kinase Chk2. It also activates signaling via the mitogen activated protein kinase (MAPK) 14 alias p38 and its substrate MAPK activated protein kinase 2 (MAPKAPK2 alias MK2) (Raman et al., 2007; Reinhardt et al., 2007). Chk2 is a central mediator of the DDR. It has various substrates implicated in cell cycle control and apoptosis, among them p53 and the Cdc25 phosphatases (Ciccia and Elledge, 2010). Furthermore, ATM controls the repair pathways of homologous recombination $(\mathrm{HR})$ and 
non-homologous end joining (NHEJ) that are employed to repair DSBs (Ciccia and Elledge, 2010).

\section{II.2.3 The ATR/Chk1 pathway}

ATR is mainly activated upon the formation of single-stranded DNA. sSDNA is recognized by replication protein A (RPA), which recruits ATR via its cofactor ATRIP (ATR-interacting protein), facilitating activation of ATR (Guo et al., 2000). Among the many substrates of ATR is the checkpoint kinase Chk1 (Cortez et al., 2001; Zhao and Piwnica-Worms, 2001). Interaction between ATR and Chk1, which is required for activation of the latter, is mediated by the adaptor protein Claspin (Kumagai et al., 2004). Like Chk2, Chk1 also serves as an important mediator of the DDR, affecting cell cycle progression, apoptosis as well as DNA repair. Like ATM, ATR also induces the p38/MK2 pathway (Raman et al., 2007; Reinhardt et al., 2007). Furthermore, p53 and Cdc25 phosphatases are subject to regulation by the ATR/Chk1 pathway, as well (Bartek and Lukas, 2003).

While mammalian cells can compensate for the loss of ATM and Chk2, ablation of ATR or of Chk1 results in embryonic lethality (Brown and Baltimore, 2003; Cimprich and Cortez, 2008; Liu et al., 2000). This striking difference has been explained by the very special functions these proteins serve within the DDR: While ATM and Chk2 are important to cope with the less frequent DSBs, the ATR/Chk1 pathway is part of a general surveillance system for DNA replication and is thus constantly activated at least at low level in S and G2 (Cimprich and Cortez, 2008). Consistently, inhibition or depletion of either ATR or Chk1 in cultured cells results in replicative stress, DNA damage and reduced cell survival (Beck et al., 2010; Forment et al., 2011; Murga et al., 2009; Syljuasen et al., 2005).

\section{II.2.4 DNA-PK}

Like ATM, DNA-PK is also activated by DSBs. Although closely related to ATM and ATR, DNA-PK phosphorylates far less proteins than the two and therefore does not play a similarly important role in the DDR. Most notably, its core function appears to consist in the stabilization of DNA ends at DSBs during repair by NHEJ (Ciccia and Elledge, 2010; Meek et al., 2008).

\section{II.2.5 Cross-signaling between ATM and ATR}

As detailed above, ATM and ATR are activated by distinct DNA lesions. However, activation of one of the two generally entails activation of the other. This is due to crosssignaling: ATR activated by ssDNA phosphorylates the histone variant $2 A X(H 2 A X)$, which induces phosphorylation of ATM (see II.2.6). Also, if stalled replication forks cannot 
be restarted or stabilized, they collapse into DSBs, again activating ATM signaling. On the other hand, when ATM becomes activated as a result of DSBs, it induces repair of the lesion by either HR or NHEJ. Opposed to NHEJ, HR involves end resection of DNA at the site of the break. This resection results in SsDNA, which activates ATR (Shiotani and Zou, 2009). Additionally, cross-signaling occurs downstream of ATR and ATM as the signaling pathways induced by both intersect at various points (Cimprich and Cortez, 2008).

\section{II.2.6 H2AX phosphorylation}

One common substrate of ATM, ATR and DNA-PK is H2AX (Burma et al., 2001; Stiff et al., 2004; Wang et al., 2005; Ward et al., 2004). H2AX differs from H2A in that it has a longer carboxy-terminal (C-terminal) tail that protrudes from the nucleosome. When activated upon DNA damage, ATM, ATR and DNA-PK phosphorylate H2AX on serine 319 , which is located proximal to the protein's C-terminus. The phosphorylated protein is called $\mathrm{yH} 2 \mathrm{AX}$. $\mathrm{H} 2 \mathrm{AX}$ phosphorylation is a very early event in the DDR, occurring within 1 min post damage induced by ionizing radiation (Rogakou et al., 1999; Rogakou et al., 1998). It is thus considered a hallmark of the DDR (Stucki and Jackson, 2006). Originally considered to be induced only by DSBs, it has recently been demonstrated that H2AX phosphorylation also occurs as a consequence of signaling originating from ssDNA (Kinner et al., 2008).

One important function of $\mathrm{H} 2 \mathrm{AX}$ is the amplification of damage signaling: $\mathrm{H} 2 \mathrm{AX}$ phosphorylation results in enhanced activation of ATM, which also spreads the $\mathrm{YH} 2 \mathrm{AX}$ mark from the site of the lesion along the DNA, forming $\mathrm{YH} 2 \mathrm{AX}$ foci (Cimprich and Cortez, 2008; Kinner et al., 2008). Furthermore, phosphorylated H2AX serves as a scaffold to recruit and assemble parts of the DNA repair machinery for HR and NHEJ (Hartlerode and Scully, 2009). Finally, H2AX appears to be essential for cell cycle regulation as cells deprived of the protein fail to induce a G2-arrest following irradiation (Fernandez-Capetillo et al., 2004).

\section{II.2.7 The cellular response to DNA damage}

\section{II.2.7.1 DNA repair}

Damage to DNA can be repaired by the cell via various pathways. DSBs are repaired by $\mathrm{HR}$ in $\mathrm{S}$ and $\mathrm{G} 2$ when sister chromatids are available. If this is not the case, repair is attempted by the more error-prone NHEJ. Both pathways are induced by ATM signaling, while NHEJ is additionally regulated by DNA-PK (Ciccia and Elledge, 2010; Sancar et al., 2004). Interestingly, it has recently been shown that Chk1 participates in the regulation of 
repair by HR, as well (Sorensen et al., 2005). Interstrand crosslinks, in contrast, induce ATR which in turn leads to the activation of the Fanconi Anemia pathway that makes use of different repair processes to remove the lesion (Branzei and Foiani, 2010; Ciccia and Elledge, 2010). For the removal of nucleoside analogs incorporated into DNA, however, the responsible DNA repair mechanism remains to be found (Ewald et al., 2008).

Stalled replication forks are initially stabilized but will eventually collapse into DSBs if replication cannot be resumed. Regulation of repair and replication at stalled replication forks will be discussed in detail below (see II.4).

Furthermore, cells evolved several mechanisms to repair lesions before it comes to replication blocks or the formation of DSBs. Such repair generally consists of the excision of one or more bases or nucleotides from the site of damage, followed by re-synthesis of the affected strand segment and is independent of ATM and ATR (Sancar et al., 2004).

\section{II.2.7.2 Induction of apoptosis in the DDR}

If the damage to the DNA is beyond repair or genotoxic stress persists for longer periods of time, the cell will eventually undergo cell death by apoptosis. Apoptosis can be triggered by strong p53 activation, leading to the upregulation of pro- and the downregulation of anti-apoptotic factors, or by p53-independent mechanisms, e.g. via E2F or c-Jun N-terminal kinases (JNKs) that are also involved in general stress signaling (Davis, 2000). Whereas for many lesions the pathway by which programmed cell death is triggered has been identified, for apoptosis induced by checkpoint failure or replicative stress the responsible pathways are still largely unknown (Ewald et al., 2008). It is important to note that tumor cells dying as a result of DNA damage do not necessarily undergo apoptosis. Rather, it has been demonstrated that a failure to elicit a proper DDR following replicative stress can entail genome fragmentation and consequently uncontrolled cell death (Durkin et al., 2006) or, alternatively, result in senescence (Bartkova et al., 2006; d'Adda di Fagagna, 2008).

\section{II.2.7.3 Cell cycle regulation}

One important function of DDR signaling is the activation of cell cycle checkpoints. While a complete and permanent arrest of the cell is only possible in $\mathrm{G} 1$, it is essential for the cell to transiently delay progression through the cell cycle when DNA damage occurs in order to allow for sufficient time for repair (Bartek et al., 2004; Massague, 2004). Generally, there are three checkpoints that can be activated by DNA damage: The G1/S, the intra-S and the G2/M checkpoint. As already mentioned, induction of either the ATM/Chk2 or the ATR/Chk1 pathway results in the activation of checkpoint signaling. This 
signaling mainly targets the cyclin dependent kinases (CDKs) that, when active, drive cell cycle progression (Ciccia and Elledge, 2010) and will be discussed in the following section.

\section{II.3 Cell cycle checkpoints}

\section{II.3.1 The G1/S checkpoint}

The master regulator of the G1/S checkpoint is the effector protein p53. p53 is a transcription factor that can be directly and indirectly activated by ATM, ATR, Chk1 and Chk2. On the one hand, phosphorylation by these kinases promotes its transcriptional activity (Meek, 2002). On the other hand, p53 is also stabilized by inhibition of the ubiquitin ligase Mdm2 that otherwise targets it for degradation (Meek and Knippschild, 2003). It then induces expression of various genes, among them p21, which potently inhibits CDK2 and CDK4, the CDKs most important for the induction of S-phase. p53 activation also impacts the activity of the Rb/E2F complex, which is essential for the maintenance of the checkpoint. Due to its central importance to the checkpoint, cancer cells harboring p53 whose transcriptional activity is inactivated by mutation fail to induce a G1/S arrest (Kastan and Bartek, 2004; Massague, 2004; Sancar et al., 2004).

\section{II.3.2 The G2/M checkpoint}

Regulation of cell cycle progression at the G2/M transition ensures that cells do not enter mitosis until replication is complete and any DNA damage is repaired. The G2/M checkpoint is also under the control of both the ATM/Chk2 and ATR/Chk1 pathway. Moreover, recent publications reveal an increasingly important role of the kinase MK2 in maintenance of the G2/M checkpoint (Manke et al., 2005; Reinhardt et al., 2007; Reinhardt et al., 2010) (see II.5.4). Opposed to G1/S, it is CDK1 alias Cdc2 that regulates progression into G2 and mitosis (Kastan and Bartek, 2004; Sancar et al., 2004).

\section{II.3.3 The intra-S-phase checkpoint}

Unlike the G1/S checkpoint, functionality of the intra-S-phase checkpoint is essential to cell viability due to its importance in the regulation of DNA replication (Segurado and Tercero, 2009). In fact, it is believed that most chromosomal rearrangements found in cancer cells originate from replication errors (Myung and Kolodner, 2002). Induction of ATR/Chk1 and ATM/Chk2 signaling results in inhibition of CDK1 and CDK2. Originally, it 
was believed that only CDK2 is involved in the regulation of S-phase progression (Kastan and Bartek, 2004). However, recent studies revealed that CDK1 is also active in S-phase and that CDK1 and CDK2 serve redundant functions during replication (Hochegger et al., 2007; Katsuno et al., 2009). The intra-S-phase checkpoint is closely connected to the regulation of DNA replication via origin firing and will be discussed in this context in more detail below (see II.4.2).

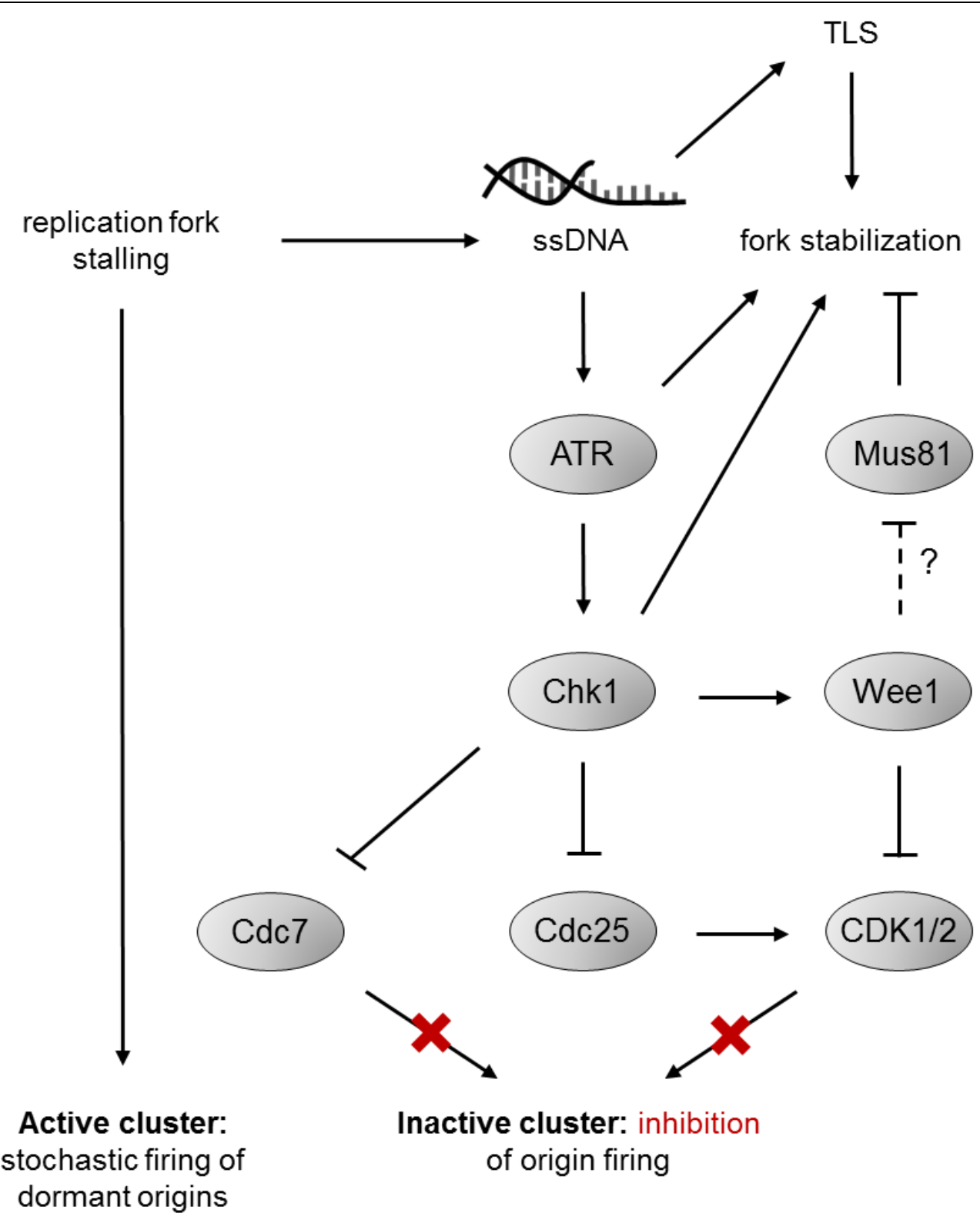

Figure II.4. Regulation of origin firing and fork stabilization upon replicative stress.

Stalling of the replication fork exposes ssDNA that activates the ATR/Chk1 pathway. ATR and Chk1 stabilize the replication fork by mechanisms not completely understood. One way is presumably the induction of chromatin-dissociation of the nuclease Mus81. That way, Mus81 is prevented from resecting the stalled fork, which would result in fork collapse and DSB formation. TLS is stimulated by SSDNA and also stabilizes the stalled fork. Chk1 inhibits origin firing in inactive replication clusters by indirectly blocking CDK1/2 activity via Wee1 and Cdc25 and by impairing Cdc7 activity. At the same time, fork stalling increases the probability of dormant origin firing in active clusters, promoting replication despite replicative stress. 


\section{II.4 Regulation of DNA replication}

\section{II.4.1 Origin firing during unperturbed S-phase}

Due to the importance of exact and reliable duplication of the genome prior to cell division and the susceptibility of this process to disturbances, DNA replication is tightly regulated. Replication is separated into three phases: Origin licensing, origin firing and strand elongation.

The licensing of replication origins takes place in $\mathrm{G} 1$. The replicative minichromosome maintenance (MCM) helicase complex, consisting of a hetero-hexameric ring of the proteins MCM2-7 and associated factors, is loaded onto DNA and forms the prereplication complex (pre-RC). The pre-RC marks origins as licensed. Only when loading is completed cells enter S-phase. This is ensured by the origin licensing checkpoint that is largely identical to the G1/S checkpoint described above (see II.3.1) and also regulated by p53 and Rb/E2F (Blow et al., 2011).

In S-phase, licensed origins of replication are fired, i.e. replication is initiated from these origins. Interestingly, origins are organized into clusters that are controlled by a timing program. This program coordinates the timing of origin firing by sequentially activating the clusters, but the details of this process are poorly understood (Blow et al., 2011).

Firing of individual origins within the clusters is mainly controlled by CDK1 and CDK2 as well as the $\mathrm{Cdc7}$ kinase. Several components of the pre-RC are phosphorylated by the CDKs and Cdc7. This phosphorylation is required for the recruitment of the replication factor Cdc45 to the pre-RC, converting it into an active helicase that starts unwinding the DNA (Masai et al., 2006). Subsequently, replication is initiated by association of the primase/DNA polymerase a complex with the pre-RC (Forsburg, 2008; Walter and Newport, 2000). Thus, replication is commenced in both directions from each origin, creating a replication bubble with a replication fork progressing along the DNA on either end. The transition to elongation then occurs by switching to the replicative polymerases $\delta$ and $\varepsilon$.

\section{II.4.2 Fork stabilization and origin firing under replicative stress}

As mentioned, the ATR/Chk1 pathway serves as a general surveillance system of replication and is therefore constantly activated to low level even during unperturbed Sphase (Shechter et al., 2004). However, replicative stress that causes replication fork stalling fully induces ATR and consequently Chk1, which activates the replication checkpoint (Branzei and Foiani, 2010; Feijoo et al., 2001). An overview of the regulation of replication under stress is provided in Figure II.4. 
One result of ATR and Chk1 induction is the stabilization of stalled forks (Durkin et al., 2006). This is essential to the cell for two reasons: Firstly, stabilized forks can rapidly resume replication once replicative stress is overcome (Branzei and Foiani, 2007). Secondly, un-stabilized forks sooner or later collapse and form DSBs, promoting genomic instability (Sorensen and Syljuasen, 2012). Many of the details as to how cells stabilize stalled forks remain elusive (Petermann and Helleday, 2010), but one way appears to be the regulation of nucleases (Sorensen and Syljuasen, 2012). One such nuclease is Mus81. It recognizes and dissects the specific DNA structure formed by stalled replication forks, inducing DSBs. When properly regulated, DSBs induced by Mus81 are essential to re-initiate replication at stalled forks that cannot be restarted by a different mechanism (Kai et al., 2005; Regairaz et al., 2011). Unrestrained Mus81 activity, however, results in extensive fork collapse and genomic instability (Dominguez-Kelly et al., 2011; Forment et al., 2011). In yeast, the Chk2-homolog Cds1 destabilizes Mus81 from chromatin following replicative stress, thus preventing fork resection by Mus81 (Kai et al., 2005). In higher eukaryotes, it is not clear which proteins are responsible for Mus81 regulation, but one candidate is the kinase Wee1 that is also regulated by ATR and Chk1 (Dominguez-Kelly
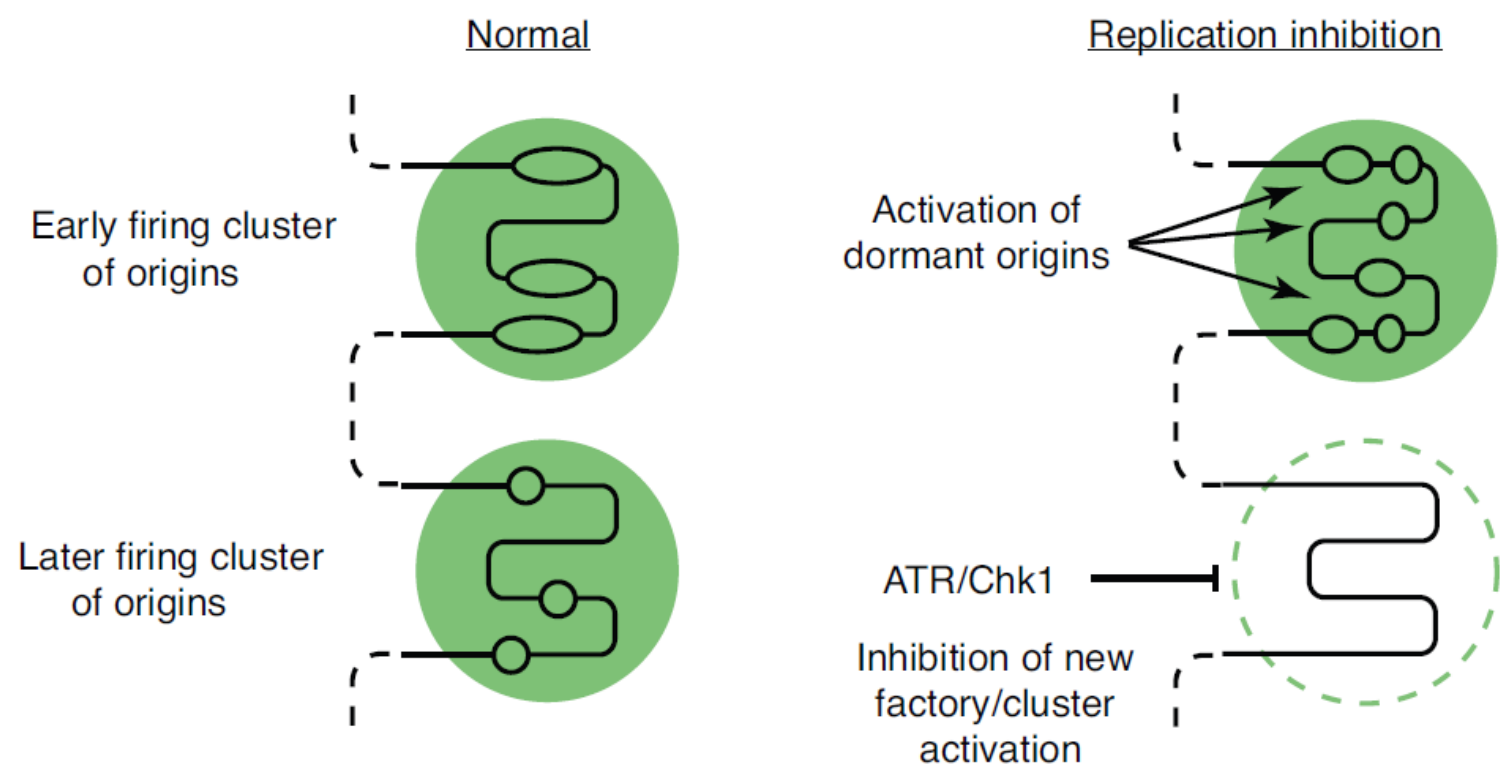

Figure II.5. A model of how origin clusters are regulated upon replicative stress.

During unperturbed replication (left), adjacent origin clusters are fired successively. DNA damage during S-phase (right) results in replication fork stalling in active origin clusters. This stalling leads to the stochastic activation of previously dormant origins that would otherwise be replicated passively. At the same time, activation of ATR and Chk1 represses the activation of new origin clusters. Illustration from Blow et al., 2011. 
et al., 2011). However, it is anticipated that the control of nucleases at stalled replication forks is more complex and involves various proteins (Sorensen and Syljuasen, 2012). A second mechanism that contributes to fork stability by promoting replication despite DNA damage is translesion synthesis, which is also influenced by the ATR/Chk1 pathway and will be discussed in detail below (see II.4.4).

Besides fork stabilization, ATR and Chk1 regulate origin firing and hence S-phase progression in response to replicative stress (Heffernan et al., 2002; Miao et al., 2003). Chk1 restrains origin firing by inhibiting CDK1 and CDK2 (Sorensen and Syljuasen, 2012). Their activity is impaired by phosphorylation on tyrosine 15 (Y15). Chk1 induces sustained CDK Y15 phosphorylation via several pathways: Firstly, Chk1 impairs pY15 removal by phosphorylating and thereby inhibiting the phosphatases Cdc25 A, B and C (Ishimi et al., 2003; Liu et al., 2006; Zhao et al., 2002). Secondly, Chk1 induces Y15 phosphorylation by the kinase Wee1 by phosphorylating and thus stabilizing Wee1 (O'Connell et al., 1997; Raleigh and O'Connell, 2000). Wee1 catalyzes the phosphorylation of Y15 on CDK1 and CDK2 (McGowan and Russell, 1993; Watanabe et al., 1995). The importance of Wee1 is emphasized by the finding that, as is the case for ATR and Chk1, loss of Wee1 is embryonically lethal (Tominaga et al., 2006) and depletion of Wee1 in cultured cells induces replicative stress (Beck et al., 2010). Thirdly, it has been demonstrated that activity of Cdc7 is also under control of the ATR/Chk1 pathway (Heffernan et al., 2007).

Given the negative regulation of origin firing by the ATR/Chk1 pathway, one should expect that replicative stress results in a reduced overall rate of origin firing. However, this is not always the case (Ge et al., 2007; Ibarra et al., 2008). This apparent contradiction can be explained by the existence of origins of replication that are not fired during replication under normal conditions. In higher eukaryotes, only 5 to $10 \%$ of the origins licensed in G1 are actually fired during replication in unperturbed cells (Blow and Ge, 2009). However, the non-fired - so-called dormant - origins are pivotal to ensure successful and complete genome replication under conditions of replicative stress and cells with reduced levels of licensed origins show increased sensitivity to DNA damage (Ge et al., 2007). It is thought that this phenomenon is due to a requirement for alternative origins of replication when replication forks stall: During unperturbed S-phase, dormant origins are replicated passively. Upon fork stalling, however, the block in replication can be rescued by the firing of a previously dormant origin at the other side of the lesion (Ge et al., 2007; Ibarra et al., 2008; Woodward et al., 2006). The inhibition of origin firing mediated by ATR and Chk1, in contrast, is only directed against origins of yet inactive replication clusters (Ge and Blow, 2010). According to the current model (Figure II.5), this 
mode of regulation promotes the rescue of stalled replication forks in active replication clusters by stochastic firing of dormant origins near the stalled fork that would otherwise be passively replicated, while initiation of replication in so far inactive clusters is prevented (Blow and Ge, 2009; Ge and Blow, 2010). It remains to be seen, however, whether other mechanisms actively contribute to the firing of dormant origins upon fork stalling, as has been suggested for ATR signaling via polo like kinase 1 (PLK1) (Cimprich and Cortez, 2008).

\section{II.4.3 Replication fork restart}

Different models exist of how replication is re-initiated at stalled forks. On the one hand, forks can restart by different ways of recombination (Petermann and Helleday, 2010). Alternatively, replication can be resumed behind the fork by re-priming (Branzei and Foiani, 2007). It has recently been demonstrated that re-priming is the method of choice after UV-induced replicative stress (Elvers et al., 2011). Finally, cells have specialized polymerases that are capable of replicating past lesions in the template strand. This process is called translesion synthesis (TLS).

\section{II.4.4 Translesion synthesis}

The replicative polymerases Pol $\delta$ and Pol $\varepsilon$ rely on an undamaged template. Therefore, they are unsuitable to synthesize DNA over lesions and stall when encountering one. In such a case, TLS polymerases can be recruited. These polymerases have a more open active site that allows them to accommodate also bulky DNA adducts (Ling et al., 2004; Prakash et al., 2005). However, they lack the proofreading 3'-5' exonuclease activity of replicative polymerases. Consequently, TLS polymerases have a much lower processivity and fidelity and their use thus goes along with a strongly increased mutation rate (Friedberg et al., 2002). TLS polymerases are Rev1, Pol I, Pol $\eta$ and Pol $\mathrm{k}$, which belong to the $Y$ family, and $\mathrm{Pol} \zeta$, a member of the $\mathrm{B}$ family that also comprises the replicative polymerases (Waters et al., 2009). According to current understanding, TLS polymerases have individual, so-called cognate lesions they are specialized for, which also explains their diversity (Waters et al., 2009). Pol $\eta$, for instance, is highly effective in bridging UVinduced pyrimidine dimers (Johnson et al., 1999) while Rev1 can bypass abasic sites (Nelson et al., 1996). Pol $\zeta$ is specialized to extend the DNA from distorted base pairs (Johnson et al., 2000). 


\section{II.4.4.1 Regulation of translesion synthesis}

TLS constitutes a DNA damage tolerance mechanism. Since it is highly error-prone, its use is tightly regulated and limited to replicative stress. An overview of the regulation of TLS is provided in Figure II.6. Immediate control of TLS is exhibited by the proliferating cell nuclear antigen (PCNA). PCNA is a homotrimeric DNA sliding clamp and cofactor of DNA polymerases. It serves as a platform for the recruitment of various factors associated with the replication fork, among them all TLS polymerases (Moldovan et al., 2007).

PCNA is subject to extensive post-translational modification. Most notably, PCNA monoubiquitinated at K164 (PCNAub1) (Hoege et al., 2002) enhances association and recruit-

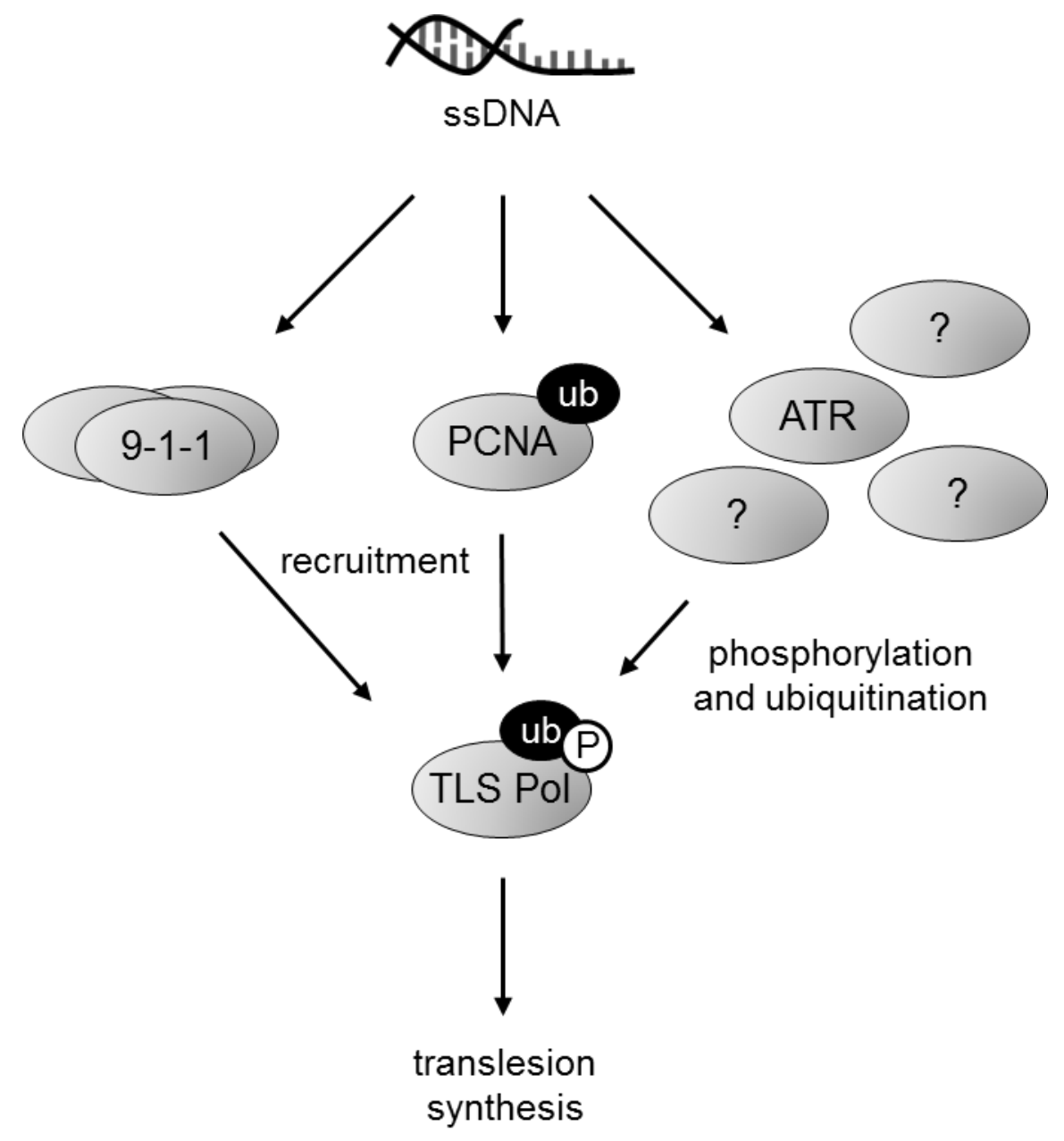

Figure II.6. Induction of translesion synthesis upon replication fork stalling.

Formation of ssDNA at stalled replication forks is the critical signal for TLS. SSDNA induces PCNA mono-ubiquitination at $\mathrm{K} 164$ and recruits the 9-1-1 complex to the replication fork. TLS polymerases localize to the fork by binding to PCNAub1 or the 9-1-1 complex. Activity of TLS polymerases is probably regulated by post-translational modification as exemplified by ATRdependent phosphorylation. 
ment of TLS polymerases to sites of DNA damage (Bienko et al., 2005; Guo et al., 2006; Kannouche et al., 2004; Stelter and Ulrich, 2003; Watanabe et al., 1995). K164 monoubiquitination of PCNA is catalyzed by the E3 ubiquitin ligase Rad18 and the E2 enzyme Rad6. The crucial stimulus appears to be SsDNA, as this is bound by Rad18, which also interacts with Rad6 and PCNA and recruits the ubiquitination machinery to the stalled fork (Moldovan et al., 2007).

ATR and Chk1 have been reported to be required for damage-induced PCNAub1 (Bi et al., 2006; Yang et al., 2008). This view, however, was recently questioned as it was argued that reduced PCNAub1 upon depletion or inhibition of ATR or Chk1 is more likely to result from increased fork collapse (Zou et al., 2003). Still, Chk1 interacts with PCNA and this interaction appears to be connected to checkpoint function, but the details remain elusive (Scorah et al., 2008; Yang and Zou, 2009).

Some TLS polymerases also show DNA damage-induced association with stalled forks that depends on the alternative sliding clamp Rad9/Hus1/Rad1 (9-1-1). The 9-1-1 complex is itself recruited to stalled forks upon ssDNA formation (Jansen et al., 2007).

Furthermore, it is known that TLS polymerases are ubiquitinated and phosphorylated on various sites and it is speculated that these modifications regulate polymerase activity (Sale et al., 2012). For Pol $\eta$, for instance, it has been demonstrated that ATR-dependent phosphorylation of S601 following UV-induced DNA damage facilitates TLS and enhances cell survival (Gohler et al., 2011). For many phosphorylations identified on TLS polymerases, however, the function is not known, which suggests that the enzymes are also regulated by yet unidentified kinases (Branzei and Foiani, 2007).

PCNA mono-ubiquitination, 9-1-1 activity and TLS polymerase modification are all induced by ssDNA at stalled replication forks (Sale et al., 2012). The fine-tuning of TLS, however, including the question how the polymerase appropriate to the lesion is chosen, requires further investigation.

\section{II.4.4.2 Global models for translesion synthesis}

There are two models as to how TLS polymerases are employed to bridge DNA lesions. According to the polymerase-switching model, the stalled replication complex directly recruits a TLS polymerase that bypasses the lesion and is then again replaced by the replicative polymerase. The gap-filling model proposes that a stalled fork is restarted by re-priming and that the resulting gap in the newly synthesized strand is post-replicatively filled by a TLS polymerase. These models are not mutually exclusive and both have found experimental support, but it remains elusive what stimuli govern the decision between the two tolerance pathways (Waters et al., 2009). 


\section{II.5 The kinase MK2}

The kinase MK2 was only mentioned briefly so far. However, it gained increasing attention in the context of the DDR in recent years and evidence is accumulating that the kinase is more central to the response than anticipated. Therefore, MK2 and the processes controlled by it will be introduced in detail in this section.

\section{II.5.1 MK2 and related MKs}

MK2 alias MAPKAPK2 is a $\mathrm{S} / \mathrm{T}$ kinase that was originally found as a factor in general stress signaling (Stokoe et al., 1992a; Stokoe et al., 1993). It is encoded by the gene MAPKAPK2. Together with the structurally related enzymes MK3 and MK5 it forms the subfamily of MAPK activated protein kinases (MAPKAPKs or MKs) within the calcium/Calmodulin-dependent protein kinase family that comprises all protein kinases downstream of MAPK signaling. MKs were found to be involved in the regulation of an unexpectedly diverse range of cellular processes (Gaestel, 2006). Unlike MK5, which is quite distinct, MK2 and MK3 are structurally similar and have almost identical substrate specificity (Clifton et al., 1996). MK2 is found from C.elegans to mammals with high structural conservation, except for its $\mathrm{N}$-terminal proline-rich region, which is only present in vertebrate MK2 (Gaestel, 2006). Most notably, MK2 is highly conserved between mouse and human with $98 \%$ sequence identity of the kinase domain on protein level (see Appendix).

\section{II.5.2 Structure and regulation of MK2}

When inactive, MK2 forms a stable complex with the MAP kinase p38 that is localized to the nucleus. MK2 harbors a nuclear localization signal in its C-terminal domain (Engel et al., 1993). Upon stress stimuli like lipopolysaccharides or anisomycin it is phosphorylated by p38 on T334, resulting in enzymatic activation. p38 also phosphorylates MK2 on T222 which is thought to further promote enzymatic activity (Engel et al., 1995; Stokoe et al., 1992a). In response to stress, the pT334-mediated activation of MK2 coincides with its nuclear export (Ben-Levy et al., 1998; Engel et al., 1998), which is facilitated by the Cterminal domain that acts as a bi-functional switch (Meng et al., 2002): T334 is located in the hinge region that connects the $\mathrm{C}$-terminal domain to the catalytic core of the enzyme (Figure II.7). When T334 is unphosphorylated, the domain is folded over the enzyme's active site and blocks it, resulting in MK2 autoinhibition. Moreover, the enzyme's nuclear export signal (NES), located in the C-terminal domain, is also concealed in this state. 


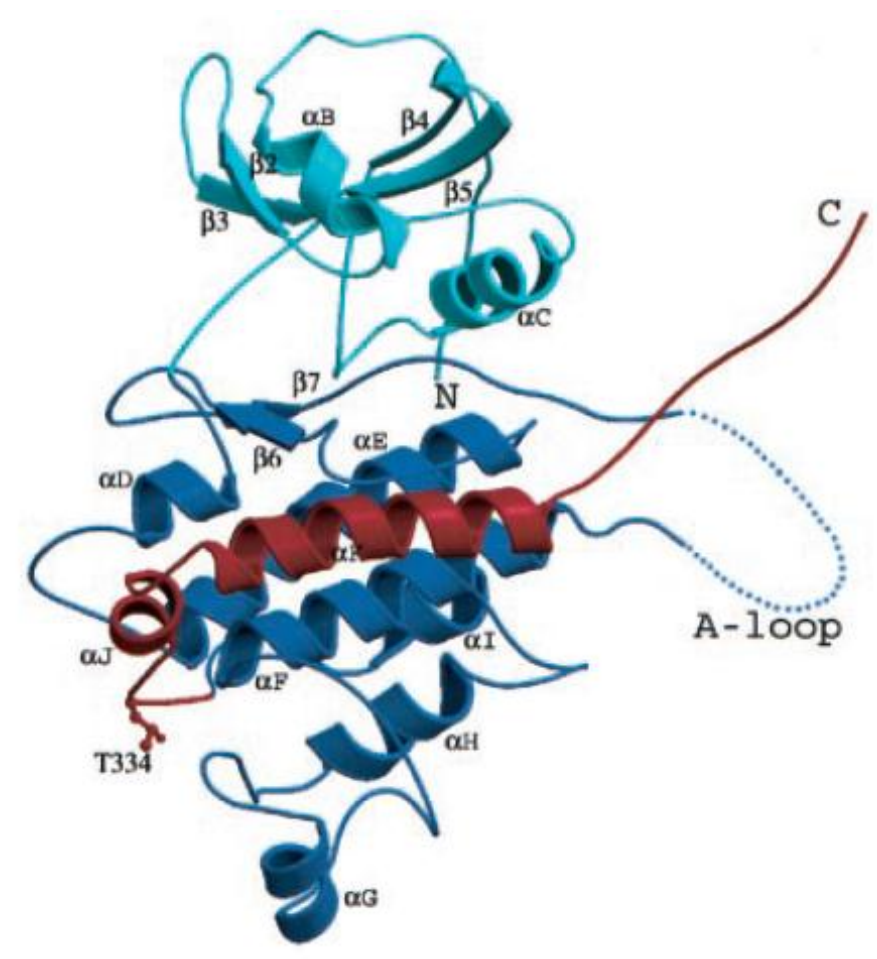

Figure II.7. 3-D structure of the kinase MK2 in its inactive state.

The C-terminal domain (red) is a bi-functional switch that is folded over the enzyme's active site and conceals the NES in the inactive state. Stress-induced phosphorylation of T334, located in the adjacent hinge region, induces a conformational change that exposes both the active site as well as the NES, promoting enzymatic activity and nuclear export. Figure from Meng et al., 2002.

Phosphorylation of T334 then induces a conformational change that exposes both the active site and the NES. In consequence, MK2 is exported to the cytoplasm by exportin-1 (Engel et al., 1998; Meng et al., 2002). It should be noted that, in addition to p38, also ERKs (extracellular signal-regulated kinases) are capable of phosphorylating and activating MK2 (Ben-Levy et al., 1995; Coxon et al., 2003).

\section{II.5.3 Substrates of MK2 and regulated processes}

Various substrates have been described for MK2, although for many of them there is only in vitro evidence. Most importantly, MK2 phosphorylates the heat shock protein of $27 \mathrm{kDa}$ (Hsp27) on S82 (Stokoe et al., 1992b), modifying its chaperone activity and presumably also influencing actin cytoskeleton dynamics (Guay et al., 1997; Rogalla et al., 1999). Furthermore, it is becoming increasingly clear that MK2 regulates gene expression by at least two mechanisms: Firstly, MK2 influences the stability of certain mRNAs by targeting various proteins involved in mRNA processing and translation. It is thus required for LPSinduced upregulation of cytokine biosynthesis, for instance (Kotlyarov et al., 1999; Winzen 
et al., 1999). Secondly, it was found that MK2 interacts with human polyhomeotic protein$2(\mathrm{HPH} 2)$ which is part of the polycomb repressive complex 1 (PRC1), and possibly contributes to PRC1-mediated gene silencing (Gaestel, 2006). This latter function of MK2 might appear contradictory to the protein's activation-coupled nuclear export. However, this export is not to be regarded as qualitatively complete. Rather, MK2 T334phosphorylation is thought to change the steady state of the nuclear-cytoplasmic distribution of the kinase, allowing for significant nuclear kinase activity (Gaestel, 2006). In support of this view, for MK3 it was shown that activating phosphorylation does not always result in immediate and complete export (Zakowski et al., 2004).

In agreement with the role of MK2 in inflammation-induced cytokine induction, MK2 knockout mice display an impaired inflammatory response and are more susceptible to bacterial infection than wild type (WT) animals (Kotlyarov et al., 1999; Lehner et al., 2002). MK2 is therefore subject to pharmacological studies as a target for the treatment of chronic inflammation (Gaestel, 2006).

\section{II.5.4 MK2 in the DNA damage response}

A first connection between the p38/MK2 pathway and the DDR was established when it was discovered that p38 activity is required for the UV-induced G2/M checkpoint by targeting Cdc25 proteins (Bulavin et al., 2001). A subsequent study identified MK2 as essential for UV-induced Cdc25 phosphorylation and demonstrated Cdc25 phosphorylation by MK2 in vitro. It extended the role of MK2 in the UV response to being required for both functional $\mathrm{G} 2 / \mathrm{M}$ - and S-phase checkpoints. The same study determined the ideal MK2 target phosphorylation to be $L / F / I-X-R-Q / S / T-L-p S / p T-\Phi$, with $\Phi$ being any hydrophobic residue (Manke et al., 2005). Within this motif, L/F/l at the -5 position (relative to the phosphorylated residue) and $R$ at the -3 position are critical for phosphorylation. Intriguingly, MK2 shares this minimal phosphorylation motif with Chk1 and Chk2. The authors therefore speculate that MK2 could be a third checkpoint kinase with similar substrates as Chk1 and Chk2 (like Cdc25s) but activated by a distinct pathway. This view gained support by over-expression studies suggesting that MK2 is essential for Cdc25A protein stability (Xiao et al., 2006).

Furthermore, it has been shown that after DNA damage Chk1 and MK2 are activated independently from each other. The activation of MK2 relies on either ATM or ATR, depending on the source of damage (Reinhardt et al., 2007). In the DDR, activation of the p38/MK2 pathway is probably mediated by thousand-and-one amino acid (TAO) kinases that were found to be direct substrates of ATM (Raman et al., 2007). Previously published results, however, failed to show any role of MK2 in IR-induced cell cycle arrest while the requirement of MK2 in the UV response is undisputed (Manke et al., 2005). This argues 
that DNA damage-induced activation of MK2 might occur downstream of ATR rather than ATM. Finally, a recent report suggests that the involvement of MK2 in the G2/M checkpoint is limited to its maintenance: While Chk1 is required for G2/M checkpoint induction, p38 and MK2 promote checkpoint maintenance by on the one hand phosphorylating Cdc25s, impairing their nuclear import, and, on the other hand, by stabilizing the mRNA of Gadd45a. Gadd45a protein binds to p38 and enhances activation of the p38/MK2 pathway, establishing a positive feedback loop. The authors also found that with regard to the G2/M checkpoint, MK2 and Chk1 can completely compensate for each other's loss when their sub-cellular localization is changed from nucleus to cytoplasm and vice versa (Reinhardt et al., 2010).

In summary, it has been established that MK2 acts as a regulator of the DDR by controlling the G2/M- and S-phase checkpoints in response to UV-induced DNA damage. Other kinds of DNA damage also induce p38/MK2 signaling and ATM and ATR appear to be involved, but the precise mode of activation is not completely understood. A number of studies suggest, however, that ATR- rather than ATM-mediated signaling results in checkpoint control by MK2. MK2 probably acts in concert with Chk1 to regulate Cdc25s and moreover is involved in checkpoint maintenance by modifying mRNA stability. It is to be expected that the activity of the p38/MK2 pathway in the DDR reaches beyond checkpoint control (Medema and Macurek, 2012), possibly influencing processes like DNA replication or DNA repair.

As is apparent from the regulation of the DDR detailed above, many factors participate in more than one response pathway, some being virtually omnipresent. While it is conceivable that the cell has to coordinate the many aspects of the DDR in order to maximize the odds of successful repair and survival, we are only beginning to understand how the different pathways integrate and how key proteins of the response are controlled. One particularly intriguing, yet unanswered question is which factors participate in the stabilization of stalled replication forks and how this process is coordinated with origin firing and DNA repair (Branzei and Foiani, 2007). A more detailed knowledge of the DDR will also promote the development of new strategies in cancer therapy.

\section{II.6 Preliminary work}

In order to obtain a comprehensive overview of kinases that are involved in the DDR to UV, a systematic robot-automated small interfering RNA (siRNA)-based high content screen using the Silencer Human Kinase siRNA Library V3 (Ambion, Life Technologies) 


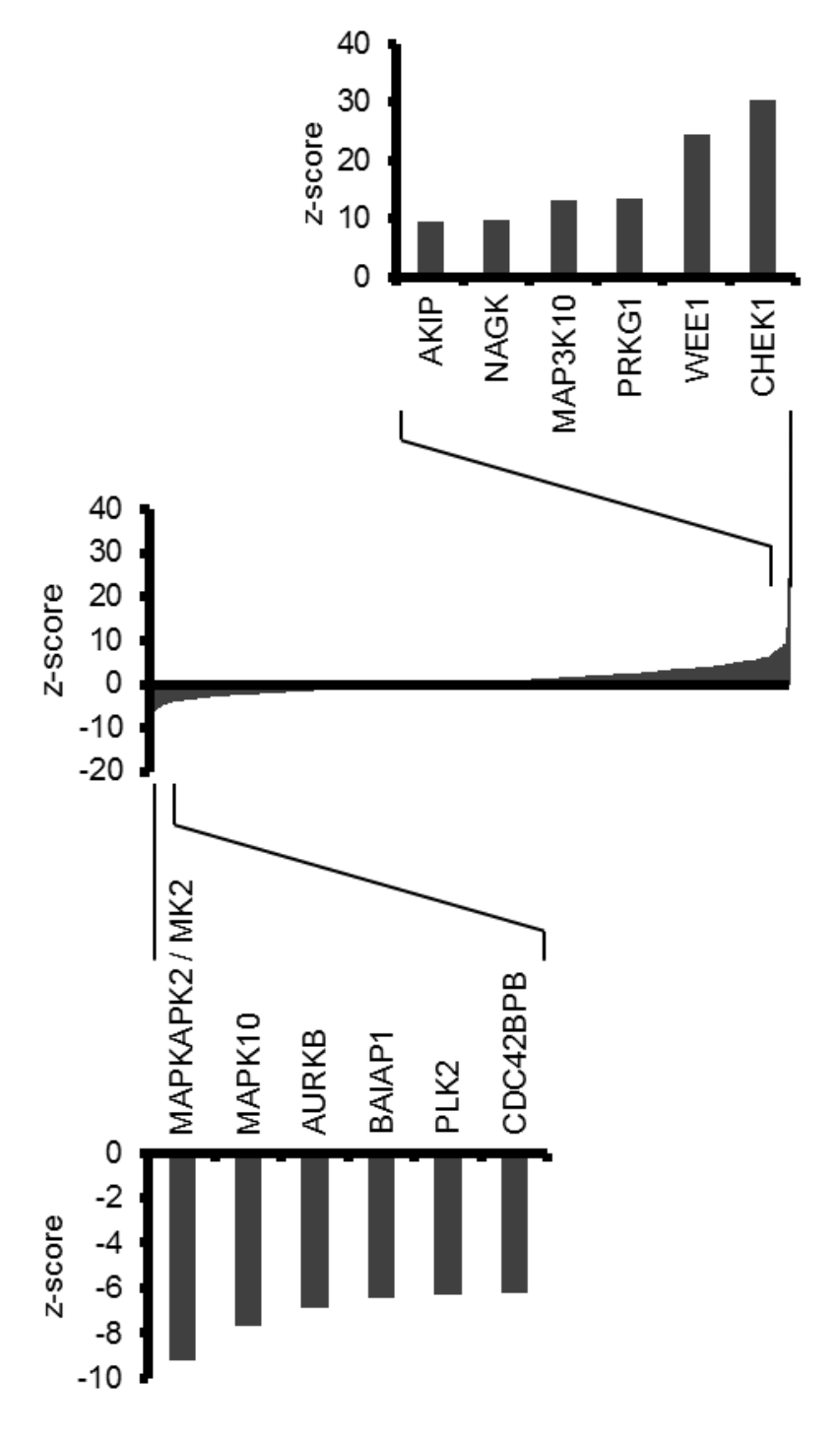

Figure II.8. RNAi kinase screening identifies kinases influencing H2AX phosphorylation following UV irradiation.

U2OS cells transfected with siRNAs were irradiated with UV-C light, fixed and stained for $\mathrm{YH} 2 \mathrm{AX}$. Target genes were ranked according to the cumulative z-score resulting from three separate siRNAs each, reflecting their impact on UV-induced H2AX phosphorylation. The six target genes with the highest and lowest cumulative z-scores are shown in magnification. MK2 was identified as the kinase whose knockdown most strongly impaired UV-induced H2AX phosphorylation. Conducted by Cathrin Bierwirth.

targeting all known kinases and their components was performed in the osteosarcoma cell line U2OS in our lab.

U2OS cells were reverse transfected with the siRNAs from the library. Cells were treated with $20 \mathrm{~J} / \mathrm{m}^{2}$ UV-C light and immunofluorescent staining was performed to detect $\mathrm{YH} 2 \mathrm{AX}$. Microscopic images were acquired and quantified automatically. The effect of each siRNA on the UV-induced H2AX phosphorylation was determined by statistical analysis using the robust z-score. All target genes were ranked according to the sum of the z-scores of all three corresponding siRNAs to identify genes that strongly influence $H 2 A X$ 
phosphorylation in response to UV-induced DNA damage. Figure II.8 presents an overview of the screen result, highlighting the top hits.

$70 \%$ of all targeted genes had a z-score between -3 and +3 , with the individual siRNAs either yielding very low individual or both positive and negative z-scores. Thus, the knockdown of those genes hardly impacted UV-induced H2AX phosphorylation. The genes coding for the kinases Wee1 and Chk1 were found to have the highest positive zscores of all targets, meaning that their knockdown strongly sensitized cells towards UVinduced DNA damage, which is in line with previous reports (see II.2.3 and II.4.2). At the other end of the scale, the screen identified the kinase MK2 to have the strongest negative effect on UV-induced $\mathrm{H} 2 \mathrm{AX}$ phosphorylation. Its knockdown resulted in strongly reduced levels of $\mathrm{yH} 2 \mathrm{AX}$.

This finding for the first time implied a function of MK2 in the phosphorylation of H2AX in the context of replicative stress, suggesting that the operating range of this kinase in the DDR goes far beyond the control of the G2/M transition.

\section{II.7 Scope of the thesis}

In spite of the in depth introduction to the DDR to replicative stress it should be noted that many details remain to be understood with regard to how cells regulate replication in response to DNA damage in S-phase. In particular, the coordination of origin firing, the stabilization of stalled forks and DNA repair is enigmatic (Blow et al., 2011). Also, while cell cycle regulation and repair pathways to cope with DSBs have been investigated in detail, the cellular response to DNA damage induced by UV irradiation and many chemotherapeutic drugs is still incompletely understood (Espinosa et al., 2003; Herrlich et al., 2008): It remains to be clarified whether repair mechanisms specialized for the removal of nucleoside analogs from the DNA exist and, if yes, how they are controlled. How is apoptosis triggered upon such damage? Which factors govern the decision by which pathway replication is re-initiated at stalled forks? A more detailed knowledge of the pathways utilized by the cell to cope with chemotherapeutics might furthermore contribute to a more effective treatment of cancer.

The kinase MK2 gained increasing attention in the context of the DDR in recent years. Based on the finding that MK2 is required for efficient phosphorylation of H2AX after UVinduced DNA damage in the screen presented above, the present work aimed to further characterize the function of MK2 in the UV response to determine whether the effect on H2AX phosphorylation also impacts cell viability and which cellular processes are responsible for this effect. The requirement of MK2 for the UV response in general and for 
cell survival after UV exposure in particular was studied both in vitro and in vivo. Furthermore, it was investigated whether MK2 plays a similar role in the response to nucleoside analogs, exemplified by the drug gemcitabine. Given that MK2 and Chk1 share the same substrate specificity but their knockdown resulted in opposite effects on UV-induced H2AX phosphorylation in the screen, a third line of experiments paralleled above studies but focused on the interplay between MK2 and Chk1.

Following up on the results obtained, it was analyzed whether MK2 participates in the regulation of origin firing and replication fork progression in response to DNA damage and whether the role of MK2 depends on TLS in this context. 


\title{
III MATERIALS
}

\author{
III.1 Technical devices
}

Table III.1. Technical Devices

Device

Company

Blotting chamber

Biozym, Hessisch Oldendorf, Germany

Cell counting chamber Neubauer improved

Centrifuge $5415 R$

Centrifuge 5810R

Centrifuge Megafuge 1.0R

Brand, Wertheim, Germany

Eppendorf, Hamburg, Germany

Eppendorf

Heraeus, Thermo Scientific, Waltham, MA, United States

Chemiluminescence imager Chemocam HR 163200

Cytometer Celigo

Intas Science Imaging Instruments,

Göttingen, Germany

DNA gel chamber

Cyntellect, San Diego, CA, United States

Biotech Service Blu, Schauenburg, Germany

Electrophoresis system, for SDS-PAGE

Electroporator GenePulser II

FACS machine Guava PCA-96 Base

System

Foil swelding machine Vacupack plus

Freezer $-20^{\circ} \mathrm{C}$

Freezer $-80^{\circ} \mathrm{C}$

Heating Block

Heating Block HLC

Ice-machine B100

Incubator for bacteria

Incubator for bacteria Minitron

Incubator for cell culture Hera Cell 150

Laminar flow cabinet Hera Safe

Liquid nitrogen tank $L S 4800$

Magnetic stirrer MR Hei-Standard

Magnetic stirrer MR3001

Microscope Axovert 40C

Microscope, automated Pathway 855

Amersham Biosciences, GE Healthcare, Little Chalfont, United Kingdom

Bio-Rad Laboratories, Hercules, CA, United

States

Millipore, Merck, Darmstadt, Germany

Krups, Groupe SEB, Lyon, France

Liebherr, Bulle, Switzerland

Heraeus, Thermo Scientific

Grant Instruments, Hillsborough, NJ, United

States

HLC Biotech, Ditabis, Pforzheim, Germany

Ziegra, Isernhagen, Germany

Memmert, Schwabach, Germany

Infors HT, Basel, Switzerland

Heraeus, Thermo Scientific, Waltham, MA,

United States

Heraeus, Thermo Scientific

Taylor-Wharton, Theodore, AL, United

States

Heidolph, Schwabach, Germany

Heidolph

Zeiss, Oberkochen, Germany

Becton Dickinson, Franklin Lakes, NJ, United States

Microscope, Axioscope 2 Plus

Zeiss

Microscope, confocal Zeiss Confocal LSM Zeiss

510 meta

Microwave

Mini Centrifuge MCF-2360

Cinex, Lippstadt, Germany

LMS, Tokyo, Japan 
PCR machine for qPCR CFX96, C1000

PCR machine Thermocycler T personal

Personal computer

pH-meter WTW-720

Pipet Multipette

Pipet, electric Portable-XP

Pipets Eppendorf Research Series 2100

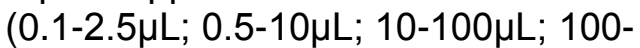

$1000 \mu \mathrm{L})$

Pipette, multichannel Research Plus

Power supply unit Powerpack P25T

Refrigerator $4^{\circ} \mathrm{C}$

Roller RM5 V-30

Rotator PTR 300

Scales Acculab ALC-6100.1

Scales LE623S

Scanner CanoScan 8600F

Sequencer, automated $A B I 3100$

Shaker PROMAX 2020

Sonication device Bioruptor

Spectrophotometer NanoDrop ND-1000

Thermomixer comfort

Timer

UV-transilluminator Intas UV system Gel Jet Imager

Vacuum pump

Vortex Genie 2

Water bath $T W 20$
Bio-Rad Laboratories

Biometra, Göttingen, Germany

Dell, Round Rock, TX, United States

WTW, Weilheim, Germany

Eppendorf

Drummond, Broomal, PA, United States

Eppendorf

Eppendorf

Biometra

Liebherr

CAT, Staufen, Germany

Grant Instruments

Sartorius, Göttingen, Germany

Sartorius

Canon, Tokyo, Japan

Applied Biosystems, Life Technologies

Heidolph

Diagenode, Liège, Belgium

PeqLab, Erlangen, Germany

Eppendorf

Oregon Scientific, Portland, OR, United

States

Intas Science Imaging Instruments

IBS Integra Biosciences, Fernwald, Germany

Scientific Industries, Bohemia, NY, United

States

Julabo Labortechnik, Seelbach, Germany

\section{III.2 Consumables}

\section{Table III.2. Consumables}

Product

8-well microscopy chamber slide

96-well plates for microscopy, clear bottom

96-well plates for microscopy, clear bottom

96-well plates for qPCR

Bacteria culture dishes

Bacteria culture vials $(14 \mathrm{~cm})$

Cell culture dishes $(10 \mathrm{~cm}, 15 \mathrm{~cm})$

Cell culture plates (6-well, 12-well)

Cell scraper $(16 \mathrm{~cm}, 25 \mathrm{~cm})$

Cover slips

Cryo tubes Cryoline

Electroporation cuvette Gene Pulser

Filter tips $(10 \mu \mathrm{L})$

Filter tips ( $20 \mu \mathrm{L}, 200 \mu \mathrm{L}, 1,000 \mu \mathrm{L})$

Glass Slides Superfrost

Parafilm

Company

Nunc, Thermo Scientific

Becton Dickinson

Corning, Corning, NY, United States

4titude, Wotton, United Kingdom

Sarstedt, Nümbrecht, Germany

Becton Dickinson

Greiner, Frickenhausen, Germany

Greiner

Sarstedt

Menzel, Thermo Scientific

Nunc, Thermo Scientific

Bio-Rad Laboratories

Starlab, Hamburg, Germany

Sarstedt

Menzel, Thermo Scientific

Brand 
Pipet tips (10 $\mu \mathrm{L}, 20-200 \mu \mathrm{L}, 1,000 \mu \mathrm{L})$

Protran nitrocellulose transfer membrane

Reaction tube $(0.2 \mathrm{~mL})$

Reaction tube $(0.5 \mathrm{~mL}, 1.5 \mathrm{~mL}, 2.0 \mathrm{~mL})$

Reaction tube $(15 \mathrm{~mL}, 50 \mathrm{~mL})$

Safe-lock reaction tube $(1.5 \mathrm{~mL})$

Sealing foil for 96 -well plate

Sterile filter

Syringe

Syringe canula (different sizes)

Transparent sealing foil for 96-well plate

Whatman paper
Greiner

Whatman, Dassel, Germany

Sarstedt

Eppendorf

Greiner

Eppendorf

Becton Dickinson

Millipore, Merck

Henke-Sass, Wolf, Tuttlingen, Germany

B.Braun, Melsungen, Germany

4titude

Whatman

\section{III.3 Chemicals and reagents}

Table III.3. Chemicals and reagents

Substance

Acetic acid

Adenosin triphosphate (ATP)

Agar

Agarose

Albumin Fraction $\mathrm{V}$ (Bovine Serum Albumine,

BSA)

Ammonium persulfate (APS)

Ammonium sulfate $\left(\left(\mathrm{NH}_{4}\right)_{2} \mathrm{SO}_{4}\right)$

Ampicillin

Bromodeoxyuridine (BrdU)

Bromophenol blue

Calcium chloride dihydrate $\left(\mathrm{CaCl}_{2} \times 2 \mathrm{H}_{2} \mathrm{O}\right)$

Chlorodeoxyuridine (CldU)

Chloroform

Complete Mini Protease Inhibitor

Crystal violet

Dimethyl sulfoxide (DMSO)

Dithiotreitol (DTT)

DNA ladder

deoxynucleotide triphosphates (dNTPs)

Ethanol $99.8 \%$

Ethanol $99.9 \%$ p.a. $(\mathrm{EtOH})$

Ethidium bromide (EtBr)

Ethylene diamine tetraacetatic acid (EDTA)

Ethylene glycol tetraacetic acid (EGTA)

Formaldehyde, $37 \%$ solution

Fugene HD

Glycerol

Glycine

Glycogen

Glycogen blue

Guava ICF Cleaning Solution

Guava Nexin
Company

Roth, Karlsruhe, Germany

Fermentas, Thermo Scientific

Sigma-Aldrich, St. Louis, MO, United

States

Roth

Roth

Roth

Roth

AppliChem, Darmstadt, Germany

Becton Dickinson

Sigma-Aldrich

Roth

Sigma-Aldrich

Roth

Roche, Basel, Schweiz

Sigma-Aldrich

AppliChem

Sigma-Aldrich

Fermentas, Thermo Scientific

Bio-Budget, Krefeld, Germany

Roth

Merck

Sigma-Aldrich

Roth

Roth

Roth

Promega, Madison, WI, United States

Roth

Roth

Fermentas, Thermo Scientific

Ambion, Life Technologies

Millipore, Merck

Millipore, Merck 


\begin{tabular}{|c|c|}
\hline Guava Viacount reagent & Millipore, Merck \\
\hline HEPES & Roth \\
\hline Hi-Di Formamide & Applied Biosystems, Life Technologies \\
\hline Hoechst 33342 (Hoechst) & Invitrogen, Life Technologies \\
\hline Hydrogen chloride $(\mathrm{HCl})$ & Roth \\
\hline Immersion oil & Zeiss \\
\hline lododeoxyuridine (IdU) & Sigma-Aldrich \\
\hline Isoamyl alcohol & Roth \\
\hline Isopropanol & Th. Geyer, Renningen, Germany \\
\hline Lipofectamine 2000 & Invitrogen, Life Technologies \\
\hline Magnesium chloride $\left(\mathrm{MgCl}_{2}\right)$ for PCR & Fermentas, Thermo Scientific \\
\hline $\begin{array}{l}\text { Magnesium chloride hexahydrate }\left(\mathrm{MgCl}_{2} \mathrm{x}\right. \\
\left.6 \mathrm{H}_{2} \mathrm{O}\right)\end{array}$ & Roth \\
\hline Methanol >99\% (MetOH) & Roth \\
\hline Nailpolish & \\
\hline Nonidet P-40 substitute (NP-40) & Sigma Aldrich \\
\hline Nuclease free water & Ambion, Life Technologies \\
\hline Peptone & Roth \\
\hline Ponceau S & Roth \\
\hline Potassium chloride (KCl) & Roth \\
\hline Potassium glutamate (KGlu) & Roth \\
\hline Potassium hydrogenphosphate $\left(\mathrm{KH}_{2} \mathrm{PO}_{4}\right)$ & Roth \\
\hline Prestained Protein Ladder & Fermentas, Thermo Scientific \\
\hline Propidium iodide (PI) & Sigma-Aldrich \\
\hline Protein-A-Sepharose (PAS) & Invitrogen, Life Technologies \\
\hline RNase inhibitor & Fermentas, Thermo Scientific \\
\hline Roti-Phenol & Roth \\
\hline Rotiphorese Gel 30 & Roth \\
\hline Sodium acetate ( $\mathrm{NaAc})$ & Roth \\
\hline Sodium bicarbonate $\left(\mathrm{NaHCO}_{3}\right)$ & Roth \\
\hline Sodium chloride $(\mathrm{NaCl})$ & Roth \\
\hline Sodium deoxycholate & Applichem \\
\hline Sodium dodecyl sulfate (SDS) & Roth \\
\hline $\begin{array}{l}\text { Sodium hydrogenphosphate heptahydrate } \\
\left(\mathrm{Na}_{2} \mathrm{HPO}_{4} \times 7 \mathrm{H}_{2} \mathrm{O}\right)\end{array}$ & Roth \\
\hline Sodium hydroxide $(\mathrm{NaOH})$ & Sigma-Aldrich \\
\hline Sodium orthovanadate & Sigma-Aldrich \\
\hline Sorbitol & Roth \\
\hline Sucrose & Sigma-Aldrich \\
\hline SYBR green & Invitrogen, Life Technologies \\
\hline Tetracycline & Sigma-Aldrich \\
\hline Tetramethylethylenediamine (TEMED) & Roth \\
\hline Thymidine & Sigma-Aldrich \\
\hline Trasylol & Bayer, Leverkusen, Germany \\
\hline Trehalose & Sigma-Aldrich \\
\hline Trisamine (Tris) & Roth \\
\hline Triton $\mathrm{X}-100$ & Applichem \\
\hline Trizol & Invitrogen, Life Technologies \\
\hline Tween 20 & Applichem \\
\hline Vectashield mounting medium & $\begin{array}{l}\text { Vector Laboratories, Burlingame, CA, } \\
\text { United States }\end{array}$ \\
\hline Yeast extract & Sigma-Aldrich \\
\hline$\beta$-Mercaptoethanol & Roth \\
\hline
\end{tabular}




\section{III.4 Buffers and solutions}

Cell lysis buffer

\begin{tabular}{lc}
\hline Urea & $2.5 \mathrm{M}$ \\
RIPA lysis buffer & $100 \%$ \\
$\Rightarrow$ for SDS PAGE, diluted with & $6 x$ \\
$1: 5$ & Laemmli
\end{tabular}

\section{ColP buffer}

\begin{tabular}{lr}
\hline Tris, $\mathrm{pH} 7.5$ & $50 \mathrm{mM}$ \\
$\mathrm{NaCl}$ & $150 \mathrm{mM}$ \\
$\mathrm{NP}-40$ & $0.20 \%$ \\
$\mathrm{Na}$ deoxycholate & $0.25 \%$ \\
Protease inhibitors & \\
dissolved in $\mathrm{H}_{2} \mathrm{O}$ &
\end{tabular}

\section{CSK buffer, pH 7.6}

\begin{tabular}{lr}
\hline HEPES & $20 \mathrm{mM}$ \\
K glutamate & $40 \mathrm{mM}$ \\
Sucrose & $300 \mathrm{mM}$ \\
$\mathrm{MgCl}_{2} \times 6 \mathrm{H}_{2} \mathrm{O}$ & $1 \mathrm{mM}$ \\
EGTA & $1 \mathrm{mM}$ \\
$\mathrm{Na}$ orthovanadate & $1 \mathrm{mM}$ \\
DTT & $1 \mathrm{mM}$ \\
Triton X-100 & $0.1 \%$ \\
Protease inhibitors & \\
dissolved in $\mathrm{H}_{2} \mathrm{O}$ &
\end{tabular}

\section{Crystal violet solution}

\begin{tabular}{lr}
\hline Crystal violet & $2.45 \mathrm{mM}$ \\
Formaldehyde & $10 \%$ \\
dissolved in $\mathrm{H}_{2} \mathrm{O}$ &
\end{tabular}

DNA gel loading buffer, $6 x$

\begin{tabular}{lr}
\hline Sucrose & $40.00 \%$ \\
Glycerin & $10.00 \%$ \\
Bromophenol blue & $0.25 \%$ \\
dissolved in $\mathrm{H}_{2} \mathrm{O}$ &
\end{tabular}

Fiber assay blocking solution

\begin{tabular}{ll}
\hline BSA & $1.0 \%$ \\
Tween 20 & $0.1 \%$ \\
dissolved in PBS &
\end{tabular}

Fiber assay fixative

\begin{tabular}{ll}
\hline MetOH & $75 \%$ \\
Acetic Acid & $25 \%$
\end{tabular}

Fiber assay spreading buffer

\begin{tabular}{lr}
\hline Tris, $\mathrm{pH} 7.4$ & $200 \mathrm{mM}$ \\
EDTA & $50 \mathrm{mM}$ \\
SDS & $0.5 \%$ \\
dissolved in $\mathrm{H}_{2} \mathrm{O}$ & \\
IF blocking solution & \\
\hline FCS & $10 \%$ \\
dissolved in PBS &
\end{tabular}

Laemmli buffer, $6 \mathbf{x}$

\begin{tabular}{lr}
\hline Tris pH 6.8 & $0.35 \mathrm{M}$ \\
Glycerin & $30.00 \%$ \\
SDS & $10.00 \%$ \\
Dithiotreitol & $9.30 \%$ \\
Bromophenol blue & $0.02 \%$ \\
dissolved in $\mathrm{H}_{2} \mathrm{O}$ & \\
\multicolumn{2}{l}{ Phophate buffered saline (PBS), $\mathbf{p H} 7.5$} \\
\hline $\mathrm{NaCl}$ & $24.00 \mathrm{mM}$ \\
$\mathrm{KCl}$ & $0.27 \mathrm{mM}$ \\
$\mathrm{Na}_{2} \mathrm{HPO}_{4} \times 7 \mathrm{H}_{2} \mathrm{O}$ & $0.81 \mathrm{mM}$ \\
$\mathrm{KH}_{2} \mathrm{PO}_{4}$ & $0.15 \mathrm{mM}$ \\
dissolved in $\mathrm{H}_{2} \mathrm{O}$ &
\end{tabular}

PBS $^{++}$

\begin{tabular}{lr}
\hline $\mathrm{NaCl}$ & $24.00 \mathrm{mM}$ \\
$\mathrm{KCl}$ & $0.27 \mathrm{mM}$ \\
$\mathrm{Na}_{2} \mathrm{HPO}_{4} \times 7 \mathrm{H}_{2} \mathrm{O}$ & $0.81 \mathrm{mM}$ \\
$\mathrm{KH}_{2} \mathrm{PO}_{4}$ & $0.15 \mathrm{mM}$ \\
$\mathrm{CaCl}_{2} \times 2 \mathrm{H}_{2} \mathrm{O}$ & $1.00 \mathrm{mM}$ \\
$\mathrm{MgCl}_{2} \times 6 \mathrm{H}_{2} \mathrm{O}$ & $0.50 \mathrm{mM}$ \\
dissolved in $\mathrm{H}_{2} \mathrm{O}$ &
\end{tabular}

\section{Ponceau S solution}

\begin{tabular}{ll}
\hline Ponceau $\mathrm{S}$ & $0.5 \%$ \\
Acetic acid & $1.0 \%$ \\
dissolved in $\mathrm{H}_{2} \mathrm{O}$ &
\end{tabular}


qPCR reaction buffer, $10 x$

Tris, $\mathrm{pH} \mathrm{8,8}$

$\left(\mathrm{NH}_{4}\right)_{2} \mathrm{SO}_{4}$

Tween 20

dissolved in $\mathrm{H}_{2} \mathrm{O}$

qPCR reaction mix, 25x

\begin{tabular}{lr}
\hline $10 x$ qPCR reaction & $1 x$ \\
buffer & \\
SybrGreen & $1: 80,000$ \\
$\mathrm{MgCl}_{2}$ & $3.0 \mathrm{mM}$ \\
Trehalose in $10 \mathrm{mM}$ & $300.0 \mathrm{mM}$ \\
Tris, pH 8,5 & \\
dNTPs & $0.2 \mathrm{mM}$ \\
Triton X-100 & $0.25 \%$ \\
Taq polymerase & $20 \mathrm{U} / \mathrm{mL}$ \\
dissolved in $\mathrm{H}_{2} \mathrm{O}$ & \\
& \\
RIPA lysis buffer, pH 7.5 & \\
\hline Triton X-100 & $1.0 \%$ \\
Na deoxycholate & $1.0 \%$ \\
SDS & $0.1 \%$ \\
NaCl & $150 \mathrm{mM}$ \\
EDTA & $10 \mathrm{mM}$ \\
Tris, pH 7.5 & $20 \mathrm{mM}$ \\
Trasylol & \\
dissolved in $\mathrm{H}_{2} \mathrm{O}$ & \\
&
\end{tabular}

SDS running buffer

\begin{tabular}{lr}
\hline Tris & $25.0 \mathrm{mM}$ \\
Glycin & $86.1 \mathrm{mM}$ \\
SDS & $3.5 \mathrm{mM}$
\end{tabular}

dissolved in $\mathrm{H}_{2} \mathrm{O}$

TAE buffer

\begin{tabular}{lr}
\hline Tris & $40 \mathrm{mM}$ \\
Acetic acid & $20 \mathrm{mM}$ \\
EDTA & $2 \mathrm{mM}$ \\
dissolved in $\mathrm{H}_{2} \mathrm{O}$ &
\end{tabular}

Tris buffered saline + Tween 20

(TBST), pH 7.6

\begin{tabular}{lr}
\hline Tris & $50 \mathrm{mM}$ \\
$\mathrm{NaCl}$ & $150 \mathrm{mM}$ \\
Tween 20 & $0.1 \%$ \\
dissolved in $\mathrm{H}_{2} \mathrm{O}$ &
\end{tabular}

Western blot blocking solution

BSA $5 \%$
dissolved in TBST

Western blot buffer, $\mathrm{pH} 8.3$

\begin{tabular}{lr}
\hline Tris & $25 \mathrm{mM}$ \\
Glycin & $192 \mathrm{mM}$ \\
MetOH & $20 \%$
\end{tabular}

\section{III.5 Chemotherapeutics and pharmacological inhibitors}

Table III.4. Chemotherapeutics

\begin{tabular}{lll} 
Name & Systematic name & Company \\
\hline Gemcitabine & $2^{\prime}, 2^{\prime}$-difluorodeoxycytidine $(\mathrm{dFdC})$ & Eli Lilly, Indianapolis, IN, \\
& & United States
\end{tabular}

Table III.5. Inhibitors

\begin{tabular}{llll} 
Name & Commercial name & Target & Company \\
\hline Chk1 Inh & SB218078 & Chk1 & Calbiochem, Merck \\
MK2 Inh & MK2 III & MK2 & Calbiochem, Merck \\
Nutlin-3 & Nutlin-3 & Mdm2 & Sigma-Aldrich \\
p38 Inh & SB203580 & p38 & Calbiochem, Merck
\end{tabular}




\section{III.6 Enzymes and buffers}

Table III.6. Enzymes and buffers

Reagent

BamHI

Buffer for M-MuLV RT, 10x

Buffer for Taq with $\mathrm{KCl}, 10 \mathrm{x}$

Buffer R

Calf Intestine Alkaline Phosphatase

Dpnl

EcoRI

EcoRV

Klenow buffer

Company

Fermentas, Thermo Scientific

New England Biolabs, Ipswich, MA, United

States

Fermentas, Thermo Scientific

Fermentas, Thermo Scientific

Fermentas, Thermo Scientific

Fermentas, Thermo Scientific

Fermentas, Thermo Scientific

Fermentas, Thermo Scientific

Klenow fragment of DNA Polymerase I

Kpnl

M-MuLV Reverse transcriptase (RT)

Pfu reaction buffer, 10x

PfuUltra DNA polymerase (PfuUltra)

RNase A

Smal

T4 ligase

T4 ligase buffer

Fermentas, Thermo Scientific

Fermentas, Thermo Scientific

Fermentas, Thermo Scientific

New England Biolabs

Stratagene, Santa Clara, CA, United States

Stratagene

Qiagen, Venlo, Netherlands

Fermentas, Thermo Scientific

Fermentas, Thermo Scientific

Fermentas, Thermo Scientific

Tango buffer

Fermentas, Thermo Scientific

Taq DNA polymerase (Taq)

Fermentas, Thermo Scientific

\section{III.7 Kits}

Table III.7. Kits

Name

Company

BigDye Terminator v3.1 Cycle Sequencing kit Click-iT EdU Alexa Fluor High-throughput

Invitrogen, Life Technologies

Imaging Assay

DermaTACS In Situ Apoptosis Detection Kit

Invitrogen, Life Technologies

Guava Check Kit

Trevigen, Gaithersburg, MD, United

States

Immobilon Western HRP Substrate Peroxide

Millipore, Merck

Solution

Invisorb Spion Plasmid Mini Kit Two

Millipore, Merck

PureYield Plasmid Midiprep System

Invitec, Stratec, Berlin, Germany

Qiaquick Gel Extraction Kit

Promega

SuperSignal West Femto Maximum

Qiagen

Sensitivity Substrate

Thermo Scientific 


\section{III.8 Oligonucleotides}

Table III.8. Small interfering RNAs

Name (identifies target) Sequence

Negative Control No. 1 undisclosed

Negative Control No. 2 CHEK1 undisclosed sense: 5'-GCAACAGUAUUUCGGUAUAtt-3' antisense: 5'-UAUACCGAAAUACUGUUGCca-3'

MAPKAPK2 \#1 (MK2\#1)

MAPKAPK2 \#2 (MK2\#2) sense: 5'-GGAUCAUGCAAUCAACAAAtt antisense: 5'-UUUGUUGAUUGCAUGAUCCaa-3' sense: 5'-CAGUAUCUGCAUUCAAUCAtt-3' antisense: 5'-UGAUUGAAUGCAGAUACUGga-3'

MDM2 sense: GCCAUUGCUUUUGAAGUUAtt-3' : antisense: 5'-UAACUUCAAAAGCAAUGGCtt-3'

POLH sense: 5'-GCACUUACAUUGAAGGGUUtt-3' antisense: 5' - AACCCUUCAAUGUAAGUGCtt-3'

Rev3L sense: 5'-GGUCUAACUCAAUAAUGGAtt-3' antisense: 5'-UCCAUUAUUGAGUUAGACCga-3'

All siRNAs are Silencer Select from Ambion, Life Technologies.

Table III.9. Primers

\begin{tabular}{|c|c|c|}
\hline Name & Sequence & Application \\
\hline CMV promoter forward & 5'-CGCAAATGGGCGGTAGGCGTG-3' & sequencing \\
\hline Sp6 promoter forward & 5'-GATTTAGGTGACACTATAG-3' & sequencing \\
\hline $\begin{array}{l}\text { MK2 T294A BamHI } \\
\text { forward }\end{array}$ & 5'-CTAAAAACGGATCCCGCCCAGAGAATGAC-3' & site-directed \\
\hline $\begin{array}{l}\text { MK2 T294A BamHI } \\
\text { reverse }\end{array}$ & 5'-TCTGGGCGGGATCCGTTTTTTAGCAGATTC-3' & $\begin{array}{l}\text { mutagenesis } \\
\text { PCR }\end{array}$ \\
\hline anchored oligo-dT & $\mathrm{dT}_{23} \mathrm{VN}$ & RT-PCR \\
\hline random nonamer & 5'-NNNNNNNNN-3' & RT-PCR \\
\hline PolH forward & 5'-CCAGTAGGCACCGAACCCAGC-3' & \multirow{2}{*}{ qPCR } \\
\hline PolH reverse & 5'-СААТTАТTССАССАСССТTССАТG-3' & \\
\hline Rev3L forward & 5'-CGCATATTCCСTACСTCCTACAGC-3' & \multirow{2}{*}{ qPCR } \\
\hline Rev3L reverse & 5'-TGGTATTTCATCTTGTTCCCACCG-3' & \\
\hline GAPDH forward & 5'-TGAAGGTCGGAGTCAACGGATTTGGT-3' & \multirow{2}{*}{ qPCR } \\
\hline GAPDH reverse & 5'-GCAGAGATGATGACCCTTTTGGCTC-3' & \\
\hline
\end{tabular}




\section{III.9 Plasmids}

Table III.10. Plasmids.

\begin{tabular}{|c|c|c|}
\hline \multirow{3}{*}{$\begin{array}{l}\text { Name } \\
\text { pcDNA3 }\end{array}$} & Source & Description \\
\hline & Invitrogen, Life & vector for \\
\hline & Technologies & $\begin{array}{l}\text { exogenous expression of proteins } \\
\text { under the control of a CMV } \\
\text { promoter in eukaryotic cells; } \\
\text { ampicillin resistance. }\end{array}$ \\
\hline pcDNA3-Myc-MK2 WT & $\begin{array}{l}\text { from Matthias Gaestel, } \\
\text { Hannover Medical } \\
\text { School }\end{array}$ & $\begin{array}{l}\text { pcDNA3 vector with open reading } \\
\text { frame coding for murine MK2 WT } \\
\text { inserted in the MCS. }\end{array}$ \\
\hline pcDNA3-Myc-MK2 K79R & $\begin{array}{l}\text { from Matthias Gaestel, } \\
\text { Hannover Medical } \\
\text { School }\end{array}$ & $\begin{array}{l}\text { Vector derived from pcDNA3-Myc- } \\
\text { MK2 WT with the exception of an } \\
\text { amino acid substitution K79 to R } \\
\text { that renders the encoded MK2 } \\
\text { protein kinase dead (MK2 KD) }\end{array}$ \\
\hline pcDNA3-Myc-MK2 T294A & $\begin{array}{l}\text { generated from } \\
\text { pcDNA3-Myc-MK2 WT } \\
\text { by site-directed } \\
\text { mutagenesis }\end{array}$ & $\begin{array}{l}\text { pcDNA3-Myc-MK2 WT engineered } \\
\text { to code for murine MK2 with the } \\
\text { amino acid substitution T294 to A } \\
\text { (MK2 T294A) }\end{array}$ \\
\hline pIRESneo & $\begin{array}{l}\text { Clontech, Takara Bio, } \\
\text { Otsu, Japan }\end{array}$ & $\begin{array}{l}\text { Expression vector for the } \\
\text { exogenous expression of proteins } \\
\text { under the control of a CMV } \\
\text { promoter in eukaryotic cells; } \\
\text { internal ribosomal entry site (IRES) } \\
\text { facilitates bicistronic transcript with } \\
\text { sequence of interest and open } \\
\text { reading frame coding for neomycin } \\
\text { resistance for selection in } \\
\text { eukaryotic cells. }\end{array}$ \\
\hline
\end{tabular}

See appendix for vector maps and MSC sequence of pIRESneo. 


\section{III.10 Antibodies}

Table III.11. Primary antibodies

\begin{tabular}{|c|c|c|c|c|}
\hline Target & Clone & $\begin{array}{l}\text { Source } \\
\text { organism }\end{array}$ & $\begin{array}{l}\text { Dilution for } \\
\text { immunoblotting }\end{array}$ & Company \\
\hline BrdU & $\mathrm{MoBu}-1$ & mouse & & Abcam, Cambridge, UK \\
\hline BrdU/CldU & $\begin{array}{l}\text { BU1/75 } \\
\text { (ICR1) }\end{array}$ & rat & & $\begin{array}{l}\text { AbDSerotec, MorphoSys, } \\
\text { Martinsried, Germany }\end{array}$ \\
\hline BrdU/ldU & B44 & mouse & & Becton Dickinson \\
\hline Cdc2 & $\mathrm{POH}-1$ & mouse & $1: 2,000$ & $\begin{array}{l}\text { Cell Signaling Technology, } \\
\text { Beverly, MA, United States }\end{array}$ \\
\hline Cdc2 pT161 & & rabbit & $1: 1,000$ & Cell Signaling Technology \\
\hline Cdc2 pY15 & & rabbit & $1: 1,000$ & Abcam \\
\hline Cdc25A & & rabbit & $1: 1,000$ & Cell Signaling Technology \\
\hline Cdc25B & & rabbit & $1: 1,000$ & Cell Signaling Technology \\
\hline Cdc25C & $5 \mathrm{H} 9$ & rabbit & $1: 1,000$ & Cell Signaling Technology \\
\hline Chk1 & 2G1D5 & mouse & $1: 1,000$ & Cell Signaling Technology \\
\hline CPDs & TDM-2 & mouse & & Cosmo Bio, Tokyo, Japan \\
\hline H2AX pS319 & JBW301 & mouse & $1: 4,000$ & Millipore, Merck \\
\hline H2AX pS319 & & rabbit & & Cell Signaling Technology \\
\hline Hsc70 & B-6 & mouse & $1: 15,000$ & $\begin{array}{l}\text { Santa Cruz Biotechnology, } \\
\text { Santa Cruz, CA, United States }\end{array}$ \\
\hline Hsp27 pS82 & E118 & rabbit & $1: 2,500$ & Abcam \\
\hline JNK pT183Y185 & & rabbit & $1: 1,000$ & Cell Signaling Technology \\
\hline Lamin B1 & $L-5$ & mouse & $1: 500$ & Zymed, Life Technologies \\
\hline MK2 & & rabbit & $1: 1,000$ & Cell Signaling Technology \\
\hline MK2 pT334 & 27B7 & rabbit & $1: 1,000$ & Cell Signaling Technology \\
\hline Mus81 & $\begin{array}{l}\text { MTA30 } \\
2 \mathrm{G} 10 / 3\end{array}$ & mouse & $1: 500$ & Santa Cruz Biotechnology \\
\hline Myc-tag & $4 \mathrm{~A} 6$ & mouse & $1: 1,000$ & Millipore, Merck \\
\hline p38 & & rabbit & $1: 1,000$ & Cell Signaling Technology \\
\hline PCNA & PC10 & mouse & $1: 500$ & Santa Cruz Biotechnology \\
\hline Wee1 & & rabbit & $1: 1,000$ & Cell Signaling Technology \\
\hline a Tubulin & B-7 & mouse & $1: 500$ & Santa Cruz Biotechnology \\
\hline$\beta$-Actin & AC-15 & mouse & $1: 20,000$ & Abcam \\
\hline$\beta$-Galactosidase & & mouse & & Promega \\
\hline
\end{tabular}


Table III.12. Secondary antibodies

Antibody

Cat. Number Company

Alexa-Fluor-488 goat anti mouse

A-11017

Invitrogen, Life Technologies

Alexa-Fluor-488 goat anti rabbit

A-11034

Invitrogen, Life Technologies

Alexa-Fluor-546 goat anti mouse

A-11003

Invitrogen, Life Technologies

Alexa-Fluor-555 goat anti rat

A-21434

Invitrogen, Life Technologies

HRP-coupled AffiniPure $F(a b ') 2$ fragment, anti mouse $\lg G(\mathrm{H}+\mathrm{L})$

Jackson Immunoresearch, HRP-coupled AffiniPure F(ab')2 fragment, anti rabbit $\lg G(\mathrm{H}+\mathrm{L})$

715-036-150 Jackson, Immunoresearch

\section{III.11 Human cell culture}

Table III.13. Human cell lines

\begin{tabular}{ll} 
Cell line & Origin \\
\hline BXPC-3 & Pancreatic adenocarcinoma \\
MIA PaCa-2 & Pancreatic adenocarcinoma \\
NHEK & Normal human epidermal \\
PaTu 8902 & Keratinocytes \\
U2OS & Pancreatic adenocarcinoma \\
\end{tabular}

Table III.14. Media and reagents for eukaryotic cell culture

Reagent

Ciprofloxacin

Dulbecco's Modified Eagle Medium (DMEM), powder

Fetal Calf Serum (FCS)

Geneticin

Keratinocyte Growth Medium

L-Glutamine

McCoy's Medium

PBS (tablets)

Penicillin/Streptomycin

RPMI Medium

Tetracyclin

Trypsin/EDTA
Company

Bayer

Gibco, Life Technologies

Gibco, Life Technologies

Gibco, Life Technologies

Promocell, Heidelberg, Germany

Gibco, Life Technologies

Gibco, Life Technologies

Gibco, Life Technologies

Gibco, Life Technologies

Gibco, Life Technologies

Gibco, Life Technologies

Gibco, Life Technologies

\section{DMEM}

$\begin{array}{lr}\text { DMEM, powder } & 10.0 \mathrm{~g} \\ \mathrm{NaHCO}_{3} & 3.7 \mathrm{~g} \\ \mathrm{HEPES} & 5.96 \mathrm{~g} \\ \text { dissolved in } \mathrm{H}_{2} \mathrm{O} & \end{array}$




\section{III.12 Bacteria}

Table III.15. Bacteria strains

Strain

Description

Company

DH10B ElectroMAX electro-competent E.coli Invitrogen, Life Technologies

\section{III.13 Bacteria culture media}

\section{YT medium}

$\begin{array}{ll}\text { Tryptone } & 1.6 \% \\ \text { Yeast extract } & 1.0 \% \\ \mathrm{NaCl} & 0.5 \%\end{array}$

2YT agar

$\begin{array}{lr}\text { YT agar } & 15 \% \\ \text { 2YT medium } & 100 \%\end{array}$

\section{III.14 Mice}

MK2/MK3 double knockout mice (Ronkina et al., 2007) were kindly provided by Matthias Gaestel, Hannover Medical School. These mice have a Black6/J background and corresponding WT animals were used as controls. All mice were bred at the animal facility of the European Neuroscience Institute in Göttingen. Mice were held in accordance with the restrictions of the German law regarding animal experiments. 


\section{III.15 Software}

Table III.16. Software

\begin{tabular}{ll} 
Name & Company \\
\hline BD Pathway Software & Becton Dickinson \\
Celigo Software & Cyntellect \\
CFX Manager Software for & Bio-Rad \\
qPCR cycler & Microsoft, Redmond, WA, United States \\
Excel & $\begin{array}{l}\text { Millipore, Merck } \\
\text { Guava Express Software }\end{array}$ \\
INTAS lab ID & Verity Software House, Topsham, ME, United States \\
ModFit LT & Peqlab \\
NanoDrop Software & Intas Science Imaging Instruments \\
UV imager software & General Public License \\
Fiji & Adobe Systems, San Jose, CA, United States \\
Adobe Photoshop CS5 & Zeiss \\
AxioVision 3.0 & Tom Hall, Ibis Therapeutics, Isis Pharmaceuticals, \\
BioEdit v7.0.5 & Carlsbad, CA, United States
\end{tabular}




\section{METHODS}

\section{IV.1 Cell biology}

\section{IV.1.1 Culturing of human cells}

Adherent human cells were cultured in coated petri dishes at $37^{\circ} \mathrm{C}, 5 \% \mathrm{CO}_{2}$ and humidified atmosphere in culture media as listed in Table III.1. The media were supplemented with 10\% FCS, $50 \mathrm{U} / \mathrm{mL}$ penicillin and streptomycin, $200 \mu \mathrm{M} \mathrm{L}$-glutamine, 2 $\mu \mathrm{g} / \mathrm{mL}$ tetracycline and $10 \mu \mathrm{g} / \mathrm{ml}$ ciprofloxacin and pre-warmed to $37^{\circ} \mathrm{C}$ prior to use. The culture media for BxPC-3 and MIA PaCa-2 cells were without ciprofloxacin. For subculture, cells were washed with PBS and detached from the dish by incubation with $0.1 \%$ trypsin/EDTA. The enzymatic activity of trypsin was stopped by addition of culture medium containing FCS. Cells were reseeded at dilutions of 1:4 to 1:20 two to three times per week, depending on the cell line. For experiments, cells were counted and seeded at the desired density. All cell culture work was performed under sterile conditions.

Table IV.1. Culture media for human cell lines

\begin{tabular}{ll} 
Cell line & Culture medium \\
\hline BxPC-3 & RPMI \\
MIA PaCa-2 & DMEM \\
NHEK & Keratinocyte growth medium \\
PaTu 8902 & DMEM \\
U2OS & DMEM
\end{tabular}

\section{IV.1.2 Freezing of cells}

For long-term storage, cells with a low passage number were used. Near-confluent cells from a $15 \mathrm{~cm}$ petri dish were detached by trypsinization as described above and centrifuged for $7 \mathrm{~min}$ at $900 \mathrm{~g}$. Cells were resuspended in pre-cooled FCS with $10 \%$ DMSO. The suspension was aliquoted into 5 cryo vials and slowly cooled down to $-80^{\circ} \mathrm{C}$. After $24 \mathrm{~h}$, cells were moved to liquid nitrogen.

\section{IV.1.3 Thawing of frozen cells}

Cell aliquots stored in liquid nitrogen were rapidly thawed and added to pre-warmed culture medium. The cells were centrifuged for $7 \mathrm{~min}$ at $900 \mathrm{~g}$, resuspended in fresh 
culture medium and seeded in a petri dish. After $24 \mathrm{~h}$, the medium was replaced by fresh medium to remove dead cells.

\section{IV.1.4 Transfection of human cells}

\section{IV.1.4.1 Transient transfection with siRNAs}

For the selective knockdown of target proteins cells were transiently reverse transfected with siRNAs 36 to $72 \mathrm{~h}$ before treatment, depending on the experimental setup. For the knockdown of Pol $\eta$ and Rev3L, a final concentration of $10 \mathrm{nM}$ per siRNA was used. For all other siRNAs, the final concentration was $5 \mathrm{nM}$. The transfection mix, consisting of siRNAs and the transfection reagent Lipofectamine 2000, was prepared in culture medium without FCS and antibiotics.

For the transfection in 12-well plates (well-surface: $9.6 \mathrm{~cm}^{2}$ ) with a final siRNA concentration of $5 \mathrm{nM}$, per well $6 \mu \mathrm{L}$ of $500 \mathrm{nM}$ siRNA and $2 \mu \mathrm{L}$ Lipofectamine 2000 were separately incubated in $100 \mu \mathrm{L}$ medium each for $5 \mathrm{~min}$. Thereupon, siRNAs and Lipofectamine 2000 were mixed and incubated for another 20 to $45 \mathrm{~min}$. The cells were prepared in $400 \mu \mathrm{L}$ culture medium with FCS and antibiotics at the desired density (which differs depending on the cell line and the experimental setup) and cells and transfection mix were added to the well at the same time. The medium was changed after $24 \mathrm{~h}$.

For transfection in 6-well plates (well-surface: $3.6 \mathrm{~cm}^{2}$ ), $12 \mu \mathrm{L}$ of $500 \mathrm{nM}$ siRNA and $4 \mu \mathrm{L}$ Lipofectamine 2000 in $200 \mu \mathrm{L}$ medium each were used per well. Cells were prepared in 1 $\mathrm{mL}$ culture medium. For transfection in 96-well plates (well-surface: $34 \mathrm{~mm}^{2}$ ), $1.5 \mu \mathrm{L}$ of $500 \mathrm{nM}$ siRNA and $0.25 \mu \mathrm{L}$ Lipofectamine 2000 in $25 \mu \mathrm{L}$ medium each were used per well. Cells were prepared in $100 \mu \mathrm{L}$ culture medium.

\section{IV.1.4.2 Transient transfection with expression vectors}

U2OS cells were transiently forward transfected with expression vectors to over-express proteins. For this, cells were seeded in 12-well plates with 80,000 cells per well $24 \mathrm{~h}$ before transfection. $1.2 \mu \mathrm{g}$ of plasmid DNA and $4 \mu \mathrm{L}$ Lipofectamine 2000 were separately incubated in $100 \mu \mathrm{L}$ medium without FCS and antibiotics each for $5 \mathrm{~min}$, then mixed, incubated for 20 to $45 \mathrm{~min}$ and added to the cells, together with $400 \mu \mathrm{L}$ complete culture medium. For transfection in 6-well plates, the double amount of plasmid DNA and Lipofectamine 2000 was used in $200 \mu \mathrm{L}$ medium each, with 180,000 cells seeded per well. The medium was changed after $4 \mathrm{~h}$ and experiments were performed 24 to $48 \mathrm{~h}$ after transfection. 
NHEK cells were also transiently forward transfected with expression vectors to overexpress proteins. To this end, cells were seeded in 12-well plates with 80,000 cells per well $24 \mathrm{~h}$ before transfection. $650 \mathrm{ng}$ of plasmid DNA and $2.5 \mu \mathrm{L}$ Fugene HD were separately incubated in $50 \mu \mathrm{L}$ medium without FCS and antibiotics each for $15 \mathrm{~min}$, then mixed, incubated for another $15 \mathrm{~min}$ and added to the cells, together with $400 \mu \mathrm{L}$ complete culture medium. The medium was changed after $4 \mathrm{~h}$ and experiments were performed $24 \mathrm{~h}$ after transfection.

\section{IV.1.4.3 Generation of stably transfected U2OS cells}

A polyclonal U2OS cell line stably expressing Myc-MK2 WT was generated by transfection with the vector pIRESneo Myc-MK2 WT. pIRESneo contains a neomycinresistance cassette that also provides resistance to aminoglycoside antibiotics. Cells that integrated the construct into their genome were thus selected with geneticin. To generate a control cell line, at the same time U2OS cells were transfected with the pIRESneo empty vector and also selected with geneticin.

U2OS cells of low passage number were used and transfection was performed as described above. $48 \mathrm{~h}$ after transfection, cells were reseeded in two $10 \mathrm{~cm}$ petri dishes with $90 \%$ and $10 \%$ of cells, respectively. $24 \mathrm{~h}$ later, selection was started by addition of $500 \mu \mathrm{g} / \mathrm{mL}$ geneticin. During the selection process, medium was changed regularly to remove dead cells. Selection was monitored daily to observe colony formation of resistant cells. Once resistant cells reached confluency, cells were passaged normally again under constant presence of geneticin.

\section{IV.1.5 Chemical treatment and UV irradiation}

Drugs for the treatment of cells were pre-dissolved in DMSO or $\mathrm{H}_{2} \mathrm{O}$. For treatment, drugs were added to pre-warmed culture medium at the concentrations detailed in Table IV.2. Controls were treated with an equivalent amount of the respective solvent.

Table IV.2. Concentrations of chemicals used for cell treatment

\begin{tabular}{lrrr} 
Chemical & Final concentration & Stock & Solvent \\
\hline Chk1 Inh & $2.5 \mu \mathrm{M}$ & $2.5 \mathrm{mM}$ & DMSO \\
Gemcitabine & different & $100 \mu \mathrm{M}$ & $\mathrm{H}_{2} \mathrm{O}$ \\
MK2 Inh & $10 \mu \mathrm{M}$ & $10 \mathrm{mM}$ & DMSO \\
Nutlin-3 & $10 \mu \mathrm{M}$ & $20 \mathrm{mM}$ & DMSO \\
p38 Inh & $10 \mu \mathrm{M}$ & $10 \mathrm{mM}$ & DMSO \\
Thymidine & $2 \mathrm{mM}$ & $200 \mathrm{mM}$ & $\mathrm{H}_{2} \mathrm{O}$
\end{tabular}

Cells were irradiated with UV light with a wavelength of either 302 (UV-B) or $254 \mathrm{~nm}$ (UVC). To this end, the medium was aspirated and cells were placed into a UV crosslinking 
chamber. From wells that were to be left untreated medium was aspirated, as well, and the wells were covered with light-proof foil during irradiation. After irradiation, the medium was replaced.

\section{IV.1.6 Cell synchronization by thymidine block}

To synchronize U2OS cells in the G1 phase of the cell cycle, thymidine was added to the culture medium to a final concentration of $2 \mathrm{mM}$ for $16 \mathrm{~h}$. An excess of thymidine causes an imbalance in the cellular nucleotide pools, which in turn inhibits nucleotide synthesis. Due to nucleotide shortage, cells cannot replicate their DNA and thus arrest at the G1/S transition. Classically, a double thymidine block (two times $16 \mathrm{~h}$ treatment with thymidine, separated by a thymidine-free period of $9 \mathrm{~h}$ ) is performed to collect all cells at the G1/S transition. This is usually necessary as cells that are in S-phase at the beginning of the first thymidine block were observed to directly arrest, thus not making it to G1. The result is a heterogeneous population with some cells arrested at G1/S, some within S-phase. To overcome this situation, cells are released and subjected to a second block.

For U2OS cells, however, we found that a single thymidine block already synchronizes the population to about $90 \%$ at the G1/S transition. A double block did not yield a better synchronization. Therefore, only a single thymidine block was used for synchronization. Cells were released from the block by washing with pre-warmed culture medium 5 times for $5 \mathrm{~min}$. Synchronization of cells and cell cycle progression after release were monitored by flow cytometry.

When combined with siRNA-mediated protein knockdown, cells were first treated with thymidine for $12 \mathrm{~h}$, then transfected with siRNA as described for $24 \mathrm{~h}$, but in the presence of thymidine. $36 \mathrm{~h}$ after starting the block, cells were released.

\section{IV.1.7 Generation of cell lysates for SDS-PAGE analysis}

For the analysis of proteins and protein modifications by SDS polyacrylamide gel electrophoresis (SDS-PAGE) (see IV.3.2), crude cell lysates were prepared. To this end, cells grown in petri dishes or well-plates were detached by scraping. The scraping and all following steps were performed on ice or at $4^{\circ} \mathrm{C}$ to minimize protein degradation. After scraping, cells were collected and centrifuged for $4 \mathrm{~min}$ at $1,400 \mathrm{~g}$, washed once with PBS and then lysed by resuspension in cell lysis buffer. Lysates with a high amount of DNA were sonicated $5 \mathrm{~min}$ to shear the DNA to ease loading. 6x Laemmli buffer was added and proteins were boiled for $5 \mathrm{~min}$ at $95^{\circ} \mathrm{C}$ and $1,400 \mathrm{rpm}$ to reduce disulfide bonds and denature proteins. 


\section{IV.1.8 Isolation of total RNA}

Total RNA from human cells was isolated for subsequent reverse transcription and quantification by real-time PCR (see IV.2.10). Cells were washed with PBS and then incubated in Trizol reagent for $5 \mathrm{~min}$ to lyse cells and dissolve nucleoprotein complexes. To separate RNA, $200 \mu \mathrm{L}$ chloroform per $1 \mathrm{~mL}$ Trizol were added and the samples were shaken. After 3 min incubation at RT, phases were separated by 15 min centrifugation at $12,000 \mathrm{~g}$ and $4^{\circ} \mathrm{C}$. RNA from the aqueous upper phase was then purified by precipitation with $500 \mu \mathrm{L}$ isopropanol per $1 \mathrm{~mL}$ Trizol. Samples were shaken, incubated for $10 \mathrm{~min}$ at $\mathrm{RT}$ and centrifuged for $10 \mathrm{~min}$ at $12,000 \mathrm{~g}$. The pellet was washed with $75 \% \mathrm{EtOH}$. To remove any residual protein contamination, the RNA was resuspended in $\mathrm{H}_{2} \mathrm{O}$ and once more precipitated in the presence of $300 \mathrm{mM} \mathrm{NaAc}$ and $50 \% \mathrm{EtOH}$. The pelleted RNA was washed with $75 \% \mathrm{EtOH}$, air-dried, resuspended in $\mathrm{H}_{2} \mathrm{O}$ and stored at $-80^{\circ} \mathrm{C}$.

\section{IV.1.9 Clonogenic assay}

Survival of cells following irradiation with UV light was assessed with a colony formation assay. Cells were reverse transfected with siRNA as described above. 48 later, cells were exposed to UV light or left untreated and subsequently reseeded in 6-well plates with 500 cells per well for untreated cells and 20,000 cells per well for irradiated cells. 15 days later, cells were rinsed with PBS and fixed in $70 \% \mathrm{MetOH}$ at $-20^{\circ} \mathrm{C}$. After fixation, cells were stained with crystal violet solution to visualize colonies.

\section{IV.1.10 Proliferation assay}

Proliferation of cells was analyzed using a Celigo cell cytometer, which measures cell confluence on the basis of contrast in bright-field light microscopy. Cells were seeded in 96-well plates at sub-confluent density in triplicates per treatment. $24 \mathrm{~h}$ after seeding, cells were subjected to treatment. Cell confluence was measured once a day, after one week usually only once every two days. For some experiments, cells were transfected with siRNA $24 \mathrm{~h}$ prior to seeding for the proliferation assay.

\section{IV.1.11 Flow cytometry}

To analyze the cell cycle profile of a cell population, cells were subjected to flow cytometry. The cells are assigned to different phases of the cell cycle based on their DNA content. To measure the DNA content, cells are stained with propidium iodide (PI), which intercalates into DNA and RNA. Thus, samples need to be treated with RNase before measurement. PI does not penetrate the membrane of living cells, therefore cells have to be fixed before staining. PI is a fluorophore that, when excited, emits light in the red 
spectrum. This emission is enhanced 20- top 30-fold upon binding to nucleic acids. For each cell, the size and fluorescence intensity is measured and the cell count is accordingly plotted against the fluorescence intensity as a measure of the DNA content.

For analysis by flow cytometry, cells were harvested by trypsinization. The growth medium was collected, as well, in order not to lose cells not attached to the bottom of the well. Cells were immediately placed on ice and all subsequent steps were performed on ice or at $4^{\circ} \mathrm{C}$.

After the harvest, cells were centrifuged for $7 \mathrm{~min}$ at $400 \mathrm{~g}$ and resuspended in $500 \mu \mathrm{L}$ $\mathrm{PBS}^{++}$. Fixation of cells was done by drop-wise addition of $1,500 \mu \mathrm{L} 100 \% \mathrm{EtOH}$ while vortexing slowly and subsequent incubation at $-20^{\circ} \mathrm{C} \mathrm{o} / \mathrm{n}$. Then, cells were centrifuged at $400 \mathrm{~g}$ for $10 \mathrm{~min}$ and rehydrated in $\mathrm{PBS}^{++}$for $10 \mathrm{~min}$. Cells were centrifuged again, resuspended in $100 \mu \mathrm{L}$ RNase $A(1 \mathrm{mg} / \mathrm{mL})$ and incubated at $37^{\circ} \mathrm{C}$ for 20 min to digest the RNA. Samples were then diluted with $400 \mu \mathrm{L}$ PBS, stained with PI (final concentration: $30 \mu \mathrm{g} / \mathrm{mL}$ ) and measured with a Guava EasyCyte flow cytometer; 10,000 events were counted per sample.

Where required, the raw data was further analyzed with the ModFit software to determine the percentage of the cell population with DNA contents corresponding to G1-, S- and G2/M-phase.

\section{IV.2 Molecular biology}

\section{IV.2.1 Transformation of electro-competent bacteria}

Bacteria are used for the rapid amplification of plasmid DNA. The electro-competent E.coli strain DH10B ElectroMAX was used for the transformation and amplification of plasmid DNA. $7 \mu \mathrm{L}$ of bacteria were transformed with $300 \mathrm{ng}$ of plasmid DNA in an electroporation cuvette using a GenePulser // electroporator. Bacteria were thereafter plated on agar plates containing ampicillin to select for transformed cells.

\section{IV.2.2 Isolation of plasmid DNA}

Prior to isolation of plasmid DNA, a single bacterial colony harboring the plasmid of interest was inoculated in $5 \mathrm{~mL}$ of $2 Y T$ medium containing $200 \mu \mathrm{g} / \mathrm{mL}$ ampicillin and incubated shaking at $37^{\circ} \mathrm{C} \mathrm{o} / \mathrm{n}$. Plasmid DNA was isolated using the Invisorb Spin Plasmid Mini Kit Two according to the manufacturer's instructions. The isolation is based on an alkaline lysis by which DNA is denatured. Upon neutralization, plasmid DNA 
renatures, but genomic DNA does not and can be precipitated by centrifugation. The plasmid DNA remains in the supernatant and is purified with silica columns.

\section{IV.2.3 Determination of nucleic acid concentrations}

DNA and RNA concentration was measured with a NanoDrop Spectrophotometer. For 1.5 $\mu \mathrm{L}$ per sample the absorption at a wavelength of $260 \mathrm{~nm}$ was determined. The resulting absorption coefficient was used to calculate the corresponding DNA or RNA concentration in the sample.

\section{IV.2.4 Polymerase chain reaction}

The polymerase chain reaction (PCR) is the method of choice for the rapid and specific amplification of DNA. In its basic form it consists of three central steps: First, the template double-stranded DNA is denatured at high temperature to form single-stranded DNA. Second, the temperature is lowered to facilitate annealing of sequence-specific primers to the template strand. Third, the annealed primers are extended and the target DNA sequence is amplified using a thermostable DNA polymerase. These steps are repeated up to 40 times, resulting in a near-exponential amplification of the target sequence.

The basic PCR reaction mix used for the amplification of DNA fragments e.g. for cloning and the corresponding PCR cycler program are shown in Table IV.3 and Table IV.4. The amount of template used depends on the kind and source of DNA. Annealing temperatures were adjusted to lie below the melting temperatures of the primers used. Elongation time was adjusted depending on the product length.

Table IV.3. Basic PCR reaction mix

\begin{tabular}{lrr} 
& Final conc. & Per reaction $[\mu \mathrm{L}]$ \\
\hline $10 x$ Taq buffer $\mathrm{KCl}^{+}$ & $1 \mathrm{x}$ & 5.00 \\
dNTPs $(20 \mathrm{mM})$ & $0.2 \mathrm{mM}$ & 0.50 \\
Primer forward $(100 \mu \mathrm{M})$ & $5 \mu \mathrm{M}$ & 2.50 \\
Primer reverse $(100 \mu \mathrm{M})$ & $5 \mu \mathrm{M}$ & 2.50 \\
$\mathrm{MgCl}_{2}(25 \mathrm{mM})$ & $3 \mathrm{mM}$ & 6.00 \\
Template DNA & customize & 1.00 \\
Taq polymerase & $1.25 \mathrm{U} /$ reaction & 0.25 \\
$\mathrm{H}_{2} \mathrm{O}$ & & ad 50.00
\end{tabular}

Table IV.4. Cycler program for basic PCR

\begin{tabular}{lr|r} 
Temperature & \multicolumn{1}{c}{ Time } \\
\hline $95^{\circ} \mathrm{C}$ & $2 \mathrm{~min}$ \\
$95^{\circ} \mathrm{C}$ & $30 \mathrm{sec}$ & \\
customize & $30 \mathrm{sec}$ & $30 \mathrm{x}$ \\
$72^{\circ} \mathrm{C}$ & customize & \\
$12^{\circ} \mathrm{C}$ & pause
\end{tabular}




\section{IV.2.5 Restriction digest of plasmid DNA}

DNA restriction with sequence-specific endonucleases was employed to analyze plasmids after site-directed mutagenesis and for cloning. As a control after site-directed mutagenesis, the restriction digest was performed in $10 \mu \mathrm{L}$ total volume, buffered with 1 $\mu \mathrm{L}$ of enzyme-specific 10x buffer and containing about $1 \mu \mathrm{g}$ plasmid DNA and $1 \mathrm{U}$ per enzyme. The reaction mix was incubated for $1 \mathrm{~h}$ at $37^{\circ} \mathrm{C}$ followed by $15 \mathrm{~min}$ at $65^{\circ} \mathrm{C}$ to inactivate the enzymes. The resulting fragments were separated by gel electrophoresis. When used for cloning, the digest was done on a larger scale to obtain sufficient material and $1 \mu \mathrm{g}$ of the product was checked for restriction efficiency by gel electrophoresis.

\section{IV.2.6 DNA gel electrophoresis}

DNA fragments were separated by electrophoresis in an agarose gel. The speed with which the DNA fragments move through the gel is inverse proportional to their size, resulting in separation.

$1 \%$ agarose gels were used. Samples were loaded onto the gel after mixing with the appropriate amount of 6x DNA loading buffer. A DNA ladder yielding bands of defined size was also loaded to facilitate size determination of the sample fragments. The DNA was stained with ethidium bromide, which was added to the gel before casting, and visualized with UV light on a Gel Jet Imager.

\section{IV.2.7 Site-directed mutagenesis}

To introduce a mutation that results in the amino acid substitution T294 to A in murine Myc-MK2 encoded by the expression vector pcDNA3 Myc-MK2 WT, site-directed mutagenesis was used. This technique employs a PCR with primers that harbor the required mutations to result in the desired amino acid substitution. Additionally, the primers are designed to introduce a new restriction site for an endonuclease into the plasmid, facilitating the identification of bacterial clones containing the desired mutated plasmid by restriction digest later on. The primers bind to one of the two plasmid strands each, the mismatching nucleotides looping out. Specific binding despite mismatches is ensured by locating the mismatches in the middle of the primer, flanked by 10 to 15 nucleotides of complementary sequence on each site. The Pfu Ultra polymerase is used for the PCR as it has a very high fidelity, reducing the probability of undesired mutations introduced during amplification.

The primers used for the PCR to construct pcDNA3 Myc-MK2 T294A are listed in Table III.9, pcDNA3 Myc-MK2 WT served as PCR template. Table IV.5 and Table IV.6 summarize the PCR reaction mix and the cycler program used. 
Table IV.5. PCR reaction mix for site-directed mutagenesis

\begin{tabular}{lr} 
Reagent & Amount \\
\hline 10x Ultra buffer & $5.0 \mu \mathrm{L}$ \\
dNTPs & $0.5 \mu \mathrm{L}$ \\
Primer forward $(10 \mu \mathrm{M})$ & $1.25 \mu \mathrm{L}$ \\
Primer reverse $(10 \mu \mathrm{M})$ & $1.25 \mu \mathrm{L}$ \\
Template DNA & $75 \mathrm{ng}$ \\
Pfu Ultra & $1.0 \mu \mathrm{L}$
\end{tabular}

Table IV.6. Cycler program for site-directed mutagenesis

\begin{tabular}{|c|c|}
\hline Temperature & Time \\
\hline $95^{\circ} \mathrm{C}$ & $2 \min$ \\
\hline $95^{\circ} \mathrm{C}$ & $30 \mathrm{sec}$ \\
\hline $55^{\circ} \mathrm{C}$ & $30 \mathrm{sec}$ \\
\hline $68^{\circ} \mathrm{C}$ & $14 \min$ \\
\hline $12^{\circ} \mathrm{C}$ & pause \\
\hline
\end{tabular}

Afterwards, samples were digested with $10 \mathrm{U} D p n l$ for $2 \mathrm{~h}$ at $37^{\circ} \mathrm{C}$. Dpnl specifically digests methylated DNA. DNA is methylated by methylation-competent bacterial strains. Therefore, only the template used for the PCR is fully methylated and will be digested. Thus, the samples are enriched for un- or hemi-methylated PCR product.

After digestion the DNA was purified by ethanol precipitation and transformed into bacteria by electroporation.

To identify clones containing the mutated plasmid, some colonies were picked, plasmid DNA isolated as described, the plasmids were digested with BamHI and the resulting fragments were separated by gel electrophoresis. Clones showing the expected fragment sizes were selected for sequencing.

\section{IV.2.8 Cloning of Myc-MK2 WT into pIRESneo}

As a preparation for the establishment of a U2OS cell line stably expressing Myc-tagged MK2 (see IV.1.4.3), the murine Myc-MK2 WT construct from the plasmid pcDNA3 MycMK2 WT was cloned into the multiple cloning site (MCS) of the vector pIRESneo.

The following cloning strategy was applied: Myc-MK2 WT was cut from the donor vector by restriction digest with Kpnl (5') and, after re-buffering, with EcoRV (3') as described (see IV.2.5). The 3' overhang resulting from restriction with Kpnl was removed by incubation with the $25 \mathrm{U}$ of Klenow fragment of DNA polymerase I in Klenow reaction buffer in the presence of $2 \mathrm{mM}$ dNTPs to produce a blunt-ended insert. The insert was purified by gel purification using the QIAquick Gel Extraction Kit according to the manufacturer's instructions.

pIRESneo was linearized with EcoRV and dephosphorylated with $4 \mathrm{U}$ calf intestine alkaline phosphatase to prevent self-ligation. The linearized vector was purified by 
phenol-chloroform extraction: An equal volume of phenol was added to the DNA, the mixture was vortexed and centrifuged for $1 \mathrm{~min}$ at 12,000 g. The upper phase was kept and mixed with an equal volume of a 24:1-chloroform/isoamylalcohol solution, vortexed, and centrifuged as before. DNA from the resulting upper phase was precipitated with $\mathrm{EtOH}$ in the presence of $300 \mathrm{mM} \mathrm{NaAc}$ at $-20^{\circ} \mathrm{C} \mathrm{o} / \mathrm{n}$, then centrifuged at $12,000 \mathrm{~g}$ at $4^{\circ} \mathrm{C}$, washed with $75 \%$ EtOH and finally resuspended in $\mathrm{H}_{2} \mathrm{O}$.

The insert was ligated into the vector with T4 DNA ligase at $8^{\circ} \mathrm{C} \mathrm{o} / \mathrm{n}$ as detailed in Table IV.7. A control ligation was performed were the insert was omitted.

Table IV.7. Ligation of Myc-MK2 WT into pIRESneo

\begin{tabular}{lr} 
& Volume $[\mu \mathrm{L}]$ \\
\hline Insert & 6.9 \\
Vector $(525 \mathrm{ng} / \mu \mathrm{L})$ & 0.1 \\
10x T4 buffer & 1.0 \\
ATP $(100 \mathrm{mM})$ & 1.0 \\
T4 ligase & 1.0
\end{tabular}

The ligation product was transformed into electro-competent bacteria and plasmid DNA was purified from resulting colonies. The plasmid DNA was control-digested with EcoRV and Smal and samples showing the expected fragment pattern were selected for sequencing.

\section{IV.2.9 Sequencing of DNA}

Plasmid DNA obtained by site-directed mutagenesis, cloning or from outside sources was sequenced using the BigDye ${ }^{\circledR}$ Terminator v3.1 Cycle Sequencing Kit. It employs a sequencing method originally established by Sanger and colleagues (Sanger et al., 1977): For the sequencing PCR, fluorescent dideoxynucleotides (ddNTPs) are added to the reaction. As these ddNTPs lack the $3^{\prime}-\mathrm{OH}$ group, elongation is terminated upon incorporation. In the sequencer, the resulting PCR products are separated in a gel matrix and the sequence is analyzed based on the fluorescence of the terminal ddNTP of each fragment size.

For the sequencing PCR, $300 \mathrm{ng}$ of plasmid DNA were mixed with the 2x sequencing mix containing the polymerase, dNTPs and fluorescently-labeled ddNTPs, and with sequencing buffer and the appropriate primer according to the manufacturer's protocol. The PCR cycler program is listed in Table IV.8. 
Table IV.8. Cycler program for sequencing PCR

\begin{tabular}{|c|c|}
\hline Temperature & Time \\
\hline $96^{\circ} \mathrm{C}$ & $2 \min$ \\
\hline $96^{\circ} \mathrm{C}$ & $10 \mathrm{sec}$ \\
\hline $55^{\circ} \mathrm{C}$ & $15 \mathrm{sec}$ \\
\hline $60^{\circ} \mathrm{C}$ & $4 \min$ \\
\hline $12^{\circ} \mathrm{C}$ & pause \\
\hline
\end{tabular}

The PCR product was purified by ethanol precipitation and resuspended in Hi-Di Formamide for sequencing using an ABI 3100 Automated Capillary Sequencer.

\section{IV.2.10 Quantification of messenger RNA by PCR}

Quantitative real-time RT-PCR was used to verify knockdown efficiencies on messenger RNA (mRNA) level. For this, RNA was purified from treated cells as described and transcribed into complementary DNA (cDNA). The levels of mRNAs from target genes were then quantified from the cDNA by quantitative real-time PCR ( $q P C R$ ) using sequence-specific primers.

\section{IV.2.10.1 Reverse transcriptase PCR}

Isolated RNA was reverse transcribed with the viral M-MuLV reverse transcriptase and the use of a mixture of anchored oligo-dT primers and random nonamers. The oligo-dT primers hybridize to the poly-A tail of mRNAs while random nonamers are used to ensure reverse transcription of tail-less RNAs.

$1 \mu \mathrm{g}$ of RNA was used per reverse transcriptase (RT) reaction and incubated with $2 \mu \mathrm{L}$ of $100 \mu \mathrm{M}$ combined primers and $4 \mu \mathrm{L}$ of dNTPs (2.5 mM each) in a total volume of $16 \mu \mathrm{L}$ for $5 \mathrm{~min}$ at $70^{\circ} \mathrm{C}$ to resolve secondary RNA structures. Then, the RT reaction mix was prepared as detailed in Table IV.9 and added to the sample. For each sample, a second RT reaction mix was prepared without reverse transcriptase to control for DNA contamination.

Table IV.9. Reaction mix for RT

\begin{tabular}{lr} 
& Volume $[\mu \mathrm{L}]$ \\
\hline 10x RT buffer & 2.0 \\
RNase inhibitor & 0.25 \\
Reverse transcriptase & 0.125 \\
$\mathrm{H}_{2} \mathrm{O}$ & 1.625
\end{tabular}

For reverse transcription, the samples were incubated for $1 \mathrm{~h}$ at $42^{\circ} \mathrm{C}$, then heated to $95^{\circ} \mathrm{C}$ for 5 min to inactivate the enzyme and $30 \mu \mathrm{L} \mathrm{H}_{2} \mathrm{O}$ were added. 


\section{IV.2.10.2 Quantitative real-time PCR}

QPCR is the method of choice for the reliable quantification of any specific DNA or (after reverse transcription) RNA template. Here, qPCR was used to quantify mRNA levels. Instead of only measuring the end-point concentration of a specific product, this method allows for monitoring the amplification of a specific DNA sequence in real time. A fluorescent dye that intercalates into double-stranded DNA, in this case SybrGreen, is used to measure the product quantity after every replication cycle. When comparing the product amounts of any gene of interest with those of a control gene (usually any gene whose expression level is considered to be stable under the treatment conditions), the relative abundance of the original template can be calculated. To specifically amplify the cDNA of an mRNA of interest, sequence-specific primers are designed in a way that a short fragment (usually 100 to $400 \mathrm{bp}$ ) of the cDNA template is amplified and that they either span exon-junctions or are located in different exons. Thus, amplification of introncontaining genomic DNA can be excluded.

For the quantification of PolH and Rev3L mRNA, cDNA template levels were normalized to GAPDH mRNA. cDNA resulting from $R T$ reactions without reverse transcriptase and qPCR samples without cDNA template served as controls. All samples were analyzed in triplicates. The qPCR reaction mix and the qPCR cycler program are detailed in Table IV.10 and Table IV.11.

Table IV.10. Reaction mix for qPCR

\begin{tabular}{lr} 
& Volume $[\mu \mathrm{L}]$ \\
\hline 25x qPCR reaction mix & 14.0 \\
Forward primer $(10 \mathrm{pmol} / \mu \mathrm{L})$ & 0.75 \\
Reverse primer $(10 \mathrm{pmol} / \mu \mathrm{L})$ & 0.75 \\
cDNA & 1.0 \\
$\mathrm{H}_{2} \mathrm{O}$ & 8.5
\end{tabular}

Table IV.11. Cycler program for sequencing PCR

\begin{tabular}{|c|c|}
\hline Temperature & Time \\
\hline $95^{\circ} \mathrm{C}$ & $2 \min$ \\
\hline $\begin{array}{l}95^{\circ} \mathrm{C} \\
60^{\circ} \mathrm{C}\end{array}$ & $\begin{array}{r}15 \mathrm{sec} \\
1 \mathrm{~min}-\mathrm{read}\end{array}$ \\
\hline
\end{tabular}

Melting curve

The fluorescence of each sample was measured once per cycle at the end of elongation ("read"). Purity of the qPCR product was controlled with a melting curve that should yield a single melting point for a specific product.

The resulting $C_{t}$ values (amplification cycle at which the fluorescence reaches the determined threshold) were used for the calculation of the relative amount of template 
using the $\Delta \Delta \mathrm{C}_{\mathrm{t}}$ method, assuming $100 \%$ amplification efficiency (i.e. a product doubling with each cycle):

relative mRNA expression

$=2^{(\Delta \mathrm{Ct} \text { ref. gene treated/target gene treated })-(\Delta \mathrm{Ct} \text { ref. gene untreated/target gene untreated })}$

\section{IV.3 Biochemistry}

\section{IV.3.1 Separation of proteins by SDS-PAGE}

Before immunoblotting to visualize proteins with specific antibodies, proteins were separated by SDS-PAGE under denaturing conditions (originally described by Laemmli (Laemmli, 1970). The separation relies on the electrophoretic mobility of the proteins. The proteins are denatured prior to electrophoresis by boiling in the presence of SDS. SDS binds to proteins, resulting in a negative overall charge that is proportional to the protein's molecular weight. Thus, when applying an electric field to the gel, the proteins travel towards the anode, with their electrophoretic mobility being a function of their molecular weight - proteins with lower weight travel faster through the pores of the gel while the progress of large proteins is slower. The vertical gels were composed of two layers, with the stacking gel the upper one and the resolving gel the lower. The stacking gel had an acrylamide/bisacrylamide concentration of $5 \%$ and a $\mathrm{pH}$ of 6.8 , facilitating the focusing of the loaded protein sample. In the resolving gel with an acrylamide/bisacrylamide concentration of 8 to $12 \%$ (depending on the desired resolution) and a $\mathrm{pH}$ of 8.8 the proteins were separated according to their molecular weight. Table IV.12 summarizes the composition of the gels. The prepared cell lysates (see IV.1.7) were loaded into the gel pockets. Additionally, pre-stained protein ladder was loaded into an adjacent pocket to monitor protein separation and to estimate their size. The gels were run at constant 80 to $130 \mathrm{~V}$ until the desired separation was reached.

Table IV.12. Composition of gels for SDS-PAGE

\begin{tabular}{lrr} 
& Stacking gel & Resolving gel \\
\hline Acrylamide-bisacrylamide & $5 \%$ & $8-12 \%$ \\
Tris, pH 6.8 $(1 \mathrm{M})$ & $126 \mathrm{mM}$ & - \\
Tris, pH $8.8(1.5 \mathrm{M})$ & - & $375 \mathrm{mM}$ \\
SDS $(10 \%)$ & $0.1 \%$ & $0.1 \%$ \\
APS (10\%) & $0.1 \%$ & $0.1 \%$ \\
TEMED & $0.3 \%$ & $0.4 \%$
\end{tabular}




\section{IV.3.2 Immunoblotting}

Proteins separated by SDS-PAGE were visualized by immunoblotting (also: western blotting) to assess either protein levels, post-translational modifications or protein interactions after co-immunoprecipitation (ColP). This method was originally published by Renart and colleagues (Renart et al., 1979) and further developed by Towbin and colleagues (Towbin et al., 1979). With this method, proteins of interest are detected with a combination of two antibodies after transfer of the proteins from the gel onto an appropriate membrane. The primary antibody is directed against an epitope of the protein of interest. Then, the membrane is incubated with a second antibody that specifically recognizes the constant region of the first antibody (thus, the secondary antibody is directed against antibodies produced by the host animal of the primary antibody, e.g. rabbit or mouse). This second antibody is coupled to horseradish peroxidase (HRP). Application of the substrate luminol that is oxidized by HRP then results in luminescence. The emitted light is recorded by a camera and thus protein bands are visualized. With this method, protein modifications can be detected, as well. To this end, primary antibodies directed against epitopes harboring the modification of interest are employed.

For wet blotting, the gel containing the proteins separated by SDS-PAGE was placed in a stack with the nitrocellulose membrane onto which the proteins were to be transferred. The stack was constructed as follows: On the anode side, two sponges followed by three pieces of Whatman paper were placed. Onto this, the nitrocellulose membrane was placed, followed by the gel. The stack was completed with three more pieces of Whatman paper and another sponge. The stack was inserted into a blotting chamber filled with western blot buffer and blotting was performed by application of an electric field with a constant voltage of $100 \mathrm{~V}$ for $90 \mathrm{~min}$ at $4^{\circ} \mathrm{C}$. After blotting, successful and equal transfer onto the membrane was controlled by staining with ponceau $\mathrm{S}$ solution.

Then, the membrane was blocked in blocking solution for $45 \mathrm{~min}$ and incubated with primary antibody in blocking solution o/n at $4^{\circ} \mathrm{C}$ or for $3 \mathrm{~h}$ at $\mathrm{RT}$. For dilutions of primary antibodies, see Table III.11. After washing three times with TBST for $5 \mathrm{~min}$ each, the membrane was incubated with secondary antibody diluted 1:10,000 in blocking solution for $1 \mathrm{~h}$ at RT. Afterwards, the membrane was again washed as before. To detect protein bands, the membrane was covered with substrate solution (Immobilon Western HRP Substrate Peroxide Solution) and luminescence was detected using a Chemocam HR 16 3200 imager. Weak signals were detected with the more sensitive substrate solution SuperSignal West Femto Maximum Sensitivity Substrate. 


\section{IV.3.3 Chromatin fractionation}

Changes of protein association to chromatin were assessed by chromatin fractionation followed by protein detection with immunoblotting. Chromatin was separated from soluble cellular protein using CSK buffer with a protocol modified from Yoshizawa-Sugata and colleagues (Yoshizawa-Sugata et al., 2005). When applied for a limited time, this buffer lyses the cell without disrupting chromatin.

Cells were seeded in 2 wells of a 6 -well plate per sample. After treatment, cells were harvested by trypsinization and put on ice. All following steps were done on ice or at $4^{\circ} \mathrm{C}$. Cells were centrifuged at $1,500 \mathrm{~g}$ for $5 \mathrm{~min}$, washed with PBS and pelleted again. Then, cells were resuspended in $40 \mu \mathrm{L}$ CSK buffer per sample and incubated on ice for $20 \mathrm{~min}$ to lyse the cells. Lysis was controlled microscopically. After centrifugation for $5 \mathrm{~min}$ at $1,500 \mathrm{~g}$, the supernatant containing the soluble proteins was collected. The pellet composed of insoluble chromatin was washed once with CSK buffer, pelleted again and resuspended in $40 \mu \mathrm{L}$ CSK buffer. Samples were sonicated to shear the DNA, $15 \mu \mathrm{L}$ of $6 \mathrm{x}$ Laemmli were added per sample and samples were boiled for $5 \mathrm{~min}$ at $95^{\circ} \mathrm{C}$ and 1,400 rpm. Protein levels were detected by immunoblotting as described (see IV.3.2).

\section{IV.3.4 Co-immunoprecipitation}

Physical interaction of MK2 with other proteins was assessed by ColP. This method utilizes the fact that proteins can be specifically precipitated using e.g. antibodies: Cell lysates are first incubated with an antibody directed against the protein whose interaction partners are to be analyzed. Then, this antibody is coupled to protein A or protein $G$ immobilized on sepharose beads. Protein $A$ and protein $G$ are of bacterial origin and recognize and bind to the constant region of certain types of antibodies. The protein of interest together with its interaction partners can then be precipitated by centrifugation. Extensive washing purifies the complex. Variation of the buffer stringency (by changing salt and detergent concentration) allows to focus the analysis on only very stable interactions or to broaden it to also include proteins that only weakly interact with the precipitated protein. Precipitated proteins can then be analyzed by e.g. immunoblotting (see IV.3.2).

To check for interaction partners of MK2, U2OS cells stably overexpressing Myc-tagged MK2 were used (see IV.1.4.3). For each CoIP, cells from a $90 \%$ confluent $15 \mathrm{~cm}$ petri dish were harvested in ColP buffer. All following steps were done on ice or at $4^{\circ} \mathrm{C}$ if not stated otherwise. The harvested cells were pressed five times through a $26 \mathrm{G}$ canula to facilitate lysis and sonicated for $10 \mathrm{~min}$. After centrifugation for $15 \mathrm{~min}$ at $12,000 \mathrm{~g}$ to remove cell debris, the lysate was incubated with $100 \mu \mathrm{L} 50 \%$ protein A sepharose (PAS) for $1 \mathrm{~h}$. This pre-clearing step is required to remove any protein that unspecifically binds 
to the sepharose beads. PAS was previously prepared by $1 \mathrm{~h}$ incubation at RT in CoIP buffer to hydrate the beads, and subsequent repetitive washing. To finish the pre-clearing, samples were centrifuged for $4 \mathrm{~min}$ at $800 \mathrm{~g}$ and the supernatant was transferred to new reaction tubes. From each sample, $50 \mu \mathrm{L}$ lysate mixed with $15 \mu \mathrm{L} 6 \mathrm{x}$ Laemmli buffer were taken as input control. The samples were split to yield one part for incubation with $2 \mu \mathrm{g}$ of mouse anti Myc-tag antibody and one part for incubation with mouse anti $\beta$-galactosidase antibody as control o/n on a rotating wheel. Then, $25 \mu \mathrm{L} 50 \%$ PAS was added per sample and samples were incubated for $2 \mathrm{~h}$. Beads with coupled antibodies were pelleted by centrifugation for $2 \mathrm{~min}$ at 3,000 rpm and washed 5 times with $500 \mu \mathrm{L}$ ColP buffer. Finally, pelleted beads were resuspended in $30 \mu \mathrm{L} 6 \mathrm{x}$ Laemmli buffer and, together with input controls, boiled for $5 \mathrm{~min}$ at $95^{\circ} \mathrm{C}$ and $1,400 \mathrm{rpm}$. Samples were analyzed by immunoblotting.

\section{IV.3.5 Immunofluorescence microscopy}

In immunofluorescence microscopy, proteins are visualized using fluorescent dyes that are coupled to antibodies: Samples are first incubated with a primary antibody specific for the protein of interest. Then, these antibodies are coupled to secondary antibodies that carry the fluorescent dye. Upon excitation, proteins can thus be visualized and identified according to the emitted fluorescence using a fluorescence microscope. As different dyes with varying fluorophores can be used, it is possible to stain and visualize more than one protein at a time.

Immunofluorescence microscopy was used to assess subcellular MK2 localization or to quantify H2AX phosphorylation.

For MK2 localization studies, U2OS cells stably expressing Myc-MK2 (see IV.1.4.3) were grown in 8-well chamber slides. All steps were done at RT. After treatment, cells were fixed in 4\% formaldehyde in PBS for 20 min, rinsed twice with PBS, then permeabilzed with $0.5 \%$ Triton X-100 in PBS for 10 min, rinsed 4 times with PBS and blocked with IF blocking solution for $10 \mathrm{~min}$. Then, cells were incubated with primary antibodies (mouse anti Myc-tag diluted 1:200 and rabbit anti YH2AX 1:50 in IF blocking solution) for $1 \mathrm{~h}$, rinsed twice with PBS, washed with PBS for $5 \mathrm{~min}$ and incubated with secondary antibodies (Alexa-Fluor-488 goat anti rabbit and Alexa-Fluor-546 goat anti mouse diluted 1:1,000 each in IF blocking solution) for $45 \mathrm{~min}$. Hoechst was added to the IF blocking solution to a final concentration of $0.5 \mu \mathrm{g} / \mathrm{mL}$ at this step to stain nuclei. Cells were again rinsed twice with PBS, washed with PBS for $5 \mathrm{~min}$ and mounted for confocal microscopy using a Zeiss Confocal LSM 510 meta microscope.

For the quantification of $\mathrm{H} 2 \mathrm{AX}$ phosphorylation, cells were grown and treated in 96-well microscopy plates in triplicates. Cells were fixed, permeabilized and blocked as above. 
For staining, mouse anti $\mathrm{yH} 2 \mathrm{AX}$ antibody was used at a dilution of $1: 1,500$ and the secondary antibody was Alexa-Fluor-546 goat anti mouse. Cells were again co-stained with Hoechst as above. After the final washing step, PBS was added to the wells and the plate was sealed with light-proof foil. Quantification of $\mathrm{yH} 2 \mathrm{AX}$ accumulation was done by high-content immunofluorescence microscopy.

\section{IV.4 High-content immunofluorescence microscopy}

H2AX phosphorylation was quantified by high-content immunofluorescence microscopy using a $B D$ Pathway 855 system. Cells grown, fixed and stained in a 96-well plate as detailed above were automatically imaged with two different channels: One detecting Hoechst, the other H2AX phosphorylation. The images were then processed using the $B D$ Pathway software. For each image, cell nuclei were identified based on the Hoechst signal. This mask was then applied to the image generated from the channel registering H2AX phosphorylation. Thus, for each nucleus, the average H2AX phosphorylation signal intensity was determined and the average intensity of all nuclei in one well was calculated. This data was further processed using Microsoft Excel to subtract background signal and to calculate the average $\mathrm{yH} 2 \mathrm{AX}$ intensity of the triplicate wells for each sample.

\section{IV.5 DNA fiber assay}

The DNA fiber assay was employed to analyze replication fork speed and origin firing in U2OS cells based on the protocol published by Jackson and Pombo (Jackson and Pombo, 1998). For this assay, cells are consecutively pulse-labeled with the nucleoside analogs 5-Chloro-2'-deoxyuridine (CldU) and 5-lodo-2'-deoxyuridine (IdU). CldU and IdU are incorporated into DNA during replication, thus marking progressing replication forks. After treatment, cells are harvested, lysed and the DNA is spread on glass slides. The samples are then fixed, blocked and stained for immunofluorescence microscopy. CldU and IdU are recognized by specific antibodies and secondary antibodies coupled to fluorescent dyes allow detection of labeled DNA tracks using a confocal microscope. An overview over resulting fork structures is given in Figure IV.1.

When a treatment is applied during e.g. the IdU label, resulting changes in replication fork speed can be assessed by measuring of IdU track length in ongoing forks. In this setting, CldU track length serves as a control. Thus, the relative replication fork speed can be calculated from the ratio of IdU over CldU track length. 


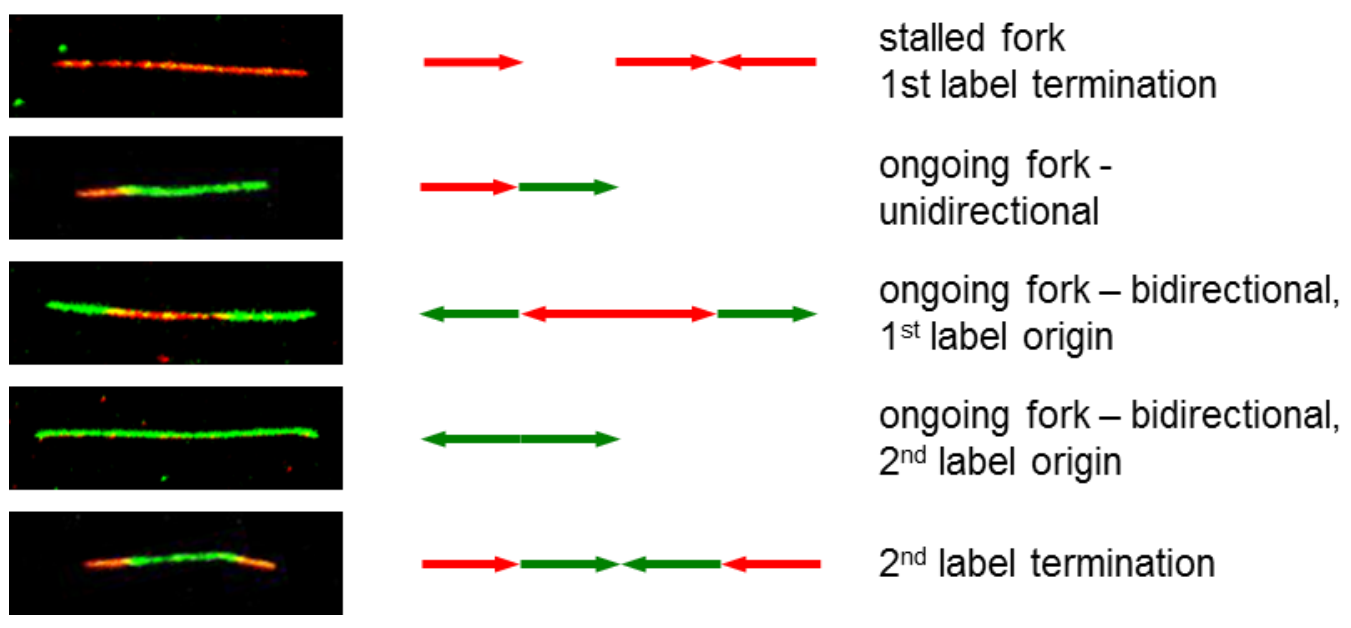

Figure IV.1. Representative images of DNA fibers obtained by DNA fiber assay and schematic representation of structures observed.

First label with CldU was detected with a red fluorescent secondary antibody, second label with IdU was detected with a green fluorescent secondary antibody. For analysis of replication fork speed, only ongoing unidirectional forks were measured. For quantification of origin firing, $1^{\text {st }}$ label origins were counted as percentage of all red-labeled fibers. Design based on: Petermann et al., 2010b.

To assess origin firing, treatment is applied throughout the labeling with both CldU and IdU. An origin that was fired during the CldU label yields a specific replication structure, which is a CldU-labeled track continued on both ends by IdU-labeled tracks ("ongoing fork, bidirectional" in Figure IV.1). The rate of origin firing during the CldU label is calculated as the percentage of such structures from all CldU-labeled structures.

For DNA fiber analysis, exponentially growing cells in 6-well plates with about $60 \%$ confluence were used with one well per treatment. All media for labeling and treatment were equilibrated to $37^{\circ} \mathrm{C}$ and appropriate $\mathrm{pH}$ for $1 \mathrm{~h}$ in a cell incubator. When inhibitors were applied, cells were pre-treated for $1 \mathrm{~h}$. Cells were then pulse-labeled with $25 \mu \mathrm{M}$ $\mathrm{CldU}$ for $20 \mathrm{~min}$. Afterwards, cells were washed once with medium and then pulse-labeled with $250 \mu \mathrm{M}$ IdU for 1 or $2 \mathrm{~h}$. IdU was used at ten times higher concentration than CldU to outcompete residual CldU and ensure that CldU is not incorporated any more during the second label. Inhibitors and gemcitabine were added at different time points depending on the experimental setting. After the IdU label, cells were harvested by scraping in PBS and placed on ice. Then, cells were pelleted by centrifugation for $7 \mathrm{~min}$ at $1,800 \mathrm{~g}$ at $4^{\circ} \mathrm{C}$ and resuspended in PBS to a final density of $5 \times 10^{5}$ cells per $\mathrm{mL}$. For each sample, a $2 \mu \mathrm{L}$ drop of suspended cells was placed at the top end of a glass slide and incubated until sticky but not dry. The drop was then mixed with $7 \mu \mathrm{L}$ fiber assay spreading buffer and incubated for $2 \mathrm{~min}$. The glass slide was tilted slightly to the effect that the drop started 
running down the slide with a speed of approximately $3 \mathrm{~cm} / \mathrm{min}$, spreading the DNA over the slide. The samples were air-dried, then fixed in fiber assay fixative for $10 \mathrm{~min}$ and stored at $4^{\circ} \mathrm{C}$ for up to one month.

For immunostaining, samples were first rehydrated by incubation in $\mathrm{H}_{2} \mathrm{O}$ twice for 5 min. Samples were rinsed with $2.5 \mathrm{M} \mathrm{HCl}$ and DNA strands were separated by treatment with $2.5 \mathrm{M} \mathrm{HCl}$ for $75 \mathrm{~min}$. Samples were rinsed twice with PBS and washed with fiber assay blocking solution two times for $5 \mathrm{~min}$. Then, samples were blocked with blocking solution for $45 \mathrm{~min}$ and thereafter incubated with the primary antibodies rat anti CldU and mouse anti IdU at a 1:1,000 dilution in blocking solution for $1 \mathrm{~h}$ at $37^{\circ} \mathrm{C}$. Samples were rinsed with PBS three times, then fixed in 4\% formaldehyde in PBS for 10 min, again rinsed with PBS three times and washed with blocking solution three times for $5 \mathrm{~min}$. Secondary antibodies Alexa-Fluor-555 goat anti rat and Alexa-Fluor-488 goat anti mouse in blocking solution at a dilution of 1:500 were applied for $2 \mathrm{~h}$ at RT. Samples were rinsed twice with PBS, washed three times for 5 min with blocking solution, rinsed twice with PBS again and mounted for confocal microscopy.

For each sample, at least ten microscopic images were taken and 100 or more structures counted and analyzed. Measurement of ongoing forks was done with Fiji, and for the counting of replicating structures the Cell Counter Plug-in for Fiji (Kurt De Vos, University of Sheffield, UK) was used. The resulting data was further processed with Microsoft Excel.

\section{IV.6 UV irradiation of mice and immunohistochemistry}

To assess the effect of MK2 depletion on apoptosis as a consequence of DNA damage in vivo, skin patches of MK2/MK3 double knock-out (DKO) mice and wt mice were irradiated with UV, the skin samples were processed for immunohistochemistry and stained for apoptotic keratinocytes.

Mice were depilated on the back and either irradiated with 2,500 J/m $\mathrm{m}^{2} \mathrm{UV}-\mathrm{B}$ light or left untreated. $24 \mathrm{~h}$ post irradiation mice were sacrificed, skin samples cut, fixed in $4 \%$ formaldehyde, embedded in paraffin and $4 \mu \mathrm{m}$ sections were cut.

To visualize apoptotic cells, terminal deoxynucleotidyl transferase UTP nick end labeling (TUNEL) was performed. This method utilizes the activity of the enzyme terminal deoxynucleotidyl transferase (TdT). In apoptosis, genomic DNA is fragmented by caspase activated DNase, producing a high number of double-stranded DNA ends. This fragmentation is a hallmark of apoptosis and can thus be used to identify apoptotic cells. In the TUNEL assay, DNA nick ends are recognized by TdT, which attaches a labeled dUTP to them. Subsequent staining allows visualization. 
For the detection of apoptotic keratinocytes in mouse skin sections, a modified TUNEL assay was employed using the DermaTACS In Situ Apoptosis Detection Kit according to the manufacturer's instructions.

As a positive control, one sample per experiment was treated with TACS nuclease which causes DNA fragmentation. A sample where TdT was omitted served as negative control. TUNEL-positive cells were quantified microscopically with an Axioscope 2 Plus and the AxioVision 3.0 image analysis software. Entire tissue sections were quantified.

\section{IV.7 Statistical analysis}

Statistical calculations were done with Microsoft Excel. Statistical significance was determined using the unpaired, two-tailed student's t-test. Significance was assumed for $p$-values below 0.05 . In figures, asterisks indicate resulting $p$-values as follows: ${ }^{*} p<0.05$, ${ }^{* *} p<0.01,{ }^{* * *} p<0.001$. n.s. $=$ not significant. $n$ in figure legends indicates the number of independent experiments. 


\section{RESULTS}

\section{V.1 MK2 in the UV response}

\section{V.1.1 MK2 knockdown or pharmacological inhibition attenuates the DNA damage response upon UV irradiation in U2OS cells}

The siRNA screen described above identified the kinase MK2 to be required for efficient H2AX phosphorylation following exposure to UV light. We used U2OS cells to validate the effect of MK2 knockdown on UV-induced H2AX phosphorylation. The human osteosarcoma cell line U2OS was chosen for subsequent experiments as it has a low level of spontaneous DNA damage and has been extensively used for studies of the DDR to UV before (Beck et al., 2010; Manke et al., 2005; Petermann et al., 2010b; Reinhardt et al., 2007; Reinhardt et al., 2010).

We depleted U2OS cells of MK2 with siRNA, UV-irradiated the cells, harvested them after $2 \mathrm{~h}$ and identified the levels of $\mathrm{\gamma H} 2 \mathrm{AX}$ by quantitative immunofluorescence. siRNAs targeting MK2 efficiently depleted cells of the protein (Figure V.1a). As was the case in the screen, MK2 depletion impaired irradiation-induced H2AX phosphorylation (Figure V.1b). Importantly, both accumulation and removal of UV-induced cyclobutane pyrimidine dimers (CPDs) were independent of MK2 knockdown (Figure V.2a), demonstrating that MK2 depletion does not interfere with the induction or repair of these lesions by DNA repair mechanisms. These results support the notion of MK2 as a central regulator of the UV response, its depletion interfering with UV-induced H2AX phosphorylation.

As an alternative measure to block MK2 activity, we made use of a pharmacological MK2 inhibitor (MK2 III; designated MK2 Inh from here on) that interferes with MK2 kinase activity by competitively binding to its active site (Anderson et al., 2007). Cells treated with UV irradiation in the presence of MK2 Inh displayed reduced levels of $\mathrm{YH} 2 \mathrm{AX}$ when compared to cells treated with DMSO (Figure V.1c), confirming the results obtained with MK2 siRNA. As was the case for MK2 depletion, inhibition of MK2 did not influence formation or removal of UV-induced DNA lesions (Figure V.2b).

In conclusion, MK2 is required for the cellular response to UV irradiation. The results suggest that MK2, being required for DNA damage-induced H2AX phosphorylation, acts in the very upstream part of the DDR but is not involved in repair of UV-induced DNA lesions. 
a

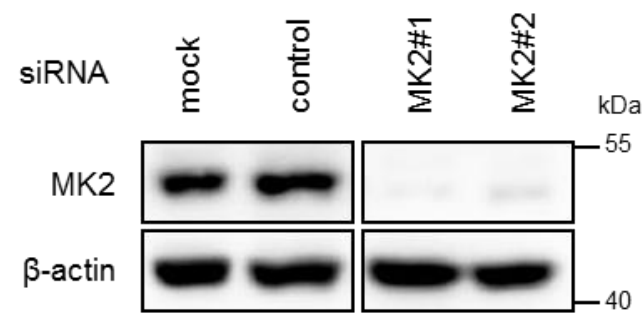

Figure V.1. Cells irradiated with UV display reduced levels of YH2AX upon MK2 inhibition and depletion.

(a) Depletion efficiencies of MK2 by siRNAmediated knockdown. Cells were harvested 48 $\mathrm{h}$ after knockdown and protein lysates were analyzed by immunoblotting. (b) MK2 depletion reduces H2AX phosphorylation after UVinduced DNA damage. U2OS cells were depleted of MK2 by siRNA-mediated knockdown, exposed to $30 \mathrm{~J} / \mathrm{m}^{2} \mathrm{UV}-\mathrm{C}$, fixed $2 \mathrm{~h}$ later, stained for immunofluorescence analysis and $\mathrm{YH} 2 \mathrm{AX}$ fluorescence intensity was quantified. Results were normalized to the control transfection and corrected for background fluorescence. (c) MK2 inhibition reduces H2AX phosphorylation after UVinduced DNA damage. U2OS cells were preincubated with MK2 Inh or DMSO for $4 \mathrm{~h}$, then irradiated with $600 \mathrm{~J} / \mathrm{m}^{2}$ UV-B. $2 \mathrm{~h}$ later, cells were fixed, processed and $\mathrm{yH} 2 \mathrm{AX}$ fluorescence intensity was quantified as in $\mathbf{b}$, conducted with Cathrin Bierwirth. b

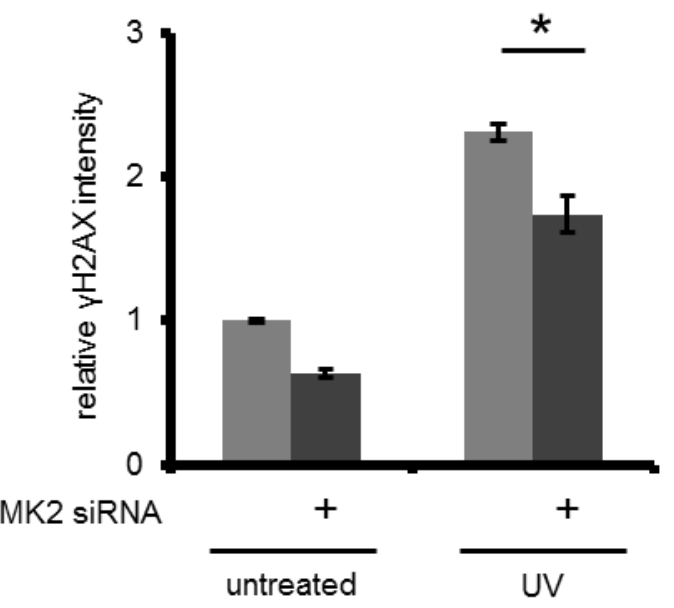

C

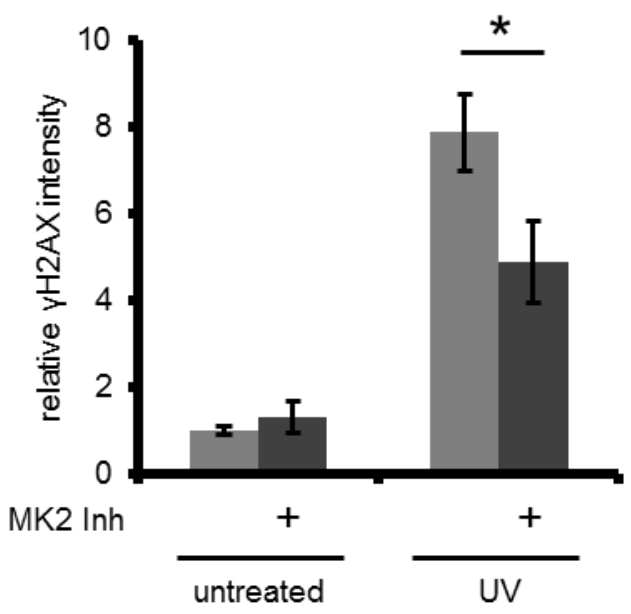

\section{V.1.2 UV-induced H2AX accumulation in keratinocytes depends on MK2 activity}

As U2OS cells are derived from mesenchymal tissue which is not naturally exposed to UV light, we additionally performed experiments with the keratinocyte cell line NHEK to determine whether UV-induced H2AX phosphorylation shows a dependence on MK2. NHEK cells irradiated with UV in the presence of MK2 Inh also accumulated less $\mathrm{YH} 2 \mathrm{AX}$ compared to DMSO-treated cells (Figure V.3a). Phosphorylation of MK2 on T334 marked MK2 activation. Reduced phosphorylation of the MK2 substrate Hsp27 at Ser 82 demonstrated that the inhibitor efficiently blocks MK2 kinase activity. As a second line of evidence, in the same cell line we compared the effect of ectopic over-expression of MK2 WT with an MK2 kinase dead mutant (MK2 KD) that is unable to bind ATP due to an amino acid substitution in the ATP binding pocket (Winzen et al., 1999). While overexpression of MK2 WT did not affect the phosphorylation of H2AX upon exposure to UV, 
over-expression of MK2 KD had a dominant negative effect and impaired $\mathrm{yH} 2 \mathrm{AX}$ accumulation, even without UV irradiation (Figure V.3b). Staining for MK2 pT334 demonstrates that both protein constructs were equally activated. These results confirm the data obtained with U2OS cells in a naturally UV-exposed cell type.

\section{V.1.3 MK2 impairs cell survival upon UV irradiation in vitro and in vivo}

The observed reduction in $\mathrm{H} 2 \mathrm{AX}$ phosphorylation upon MK2 depletion raised the question whether MK2 might also influence cell survival following UV-induced DNA damage since a modulated DDR is likely to result in changes in death signaling. To test this, we performed clonogenic assays after UV irradiation with cells depleted of MK2 or of Mdm2 as control (Figure V.4). Mdm2 knockdown reduced colony formation in untreated cells and improved cell survival after exposure to UV light. This has been observed before and can be attributed to an accumulation of p53 and subsequent cell cycle arrest, protecting the cells from UV-induced DNA damage (Kranz et al., 2008). In contrast, MK2 depletion did not affect colony formation in untreated cells but led to a marked increase in colonies after UV irradiation, compared to cells transfected with control siRNA.

a

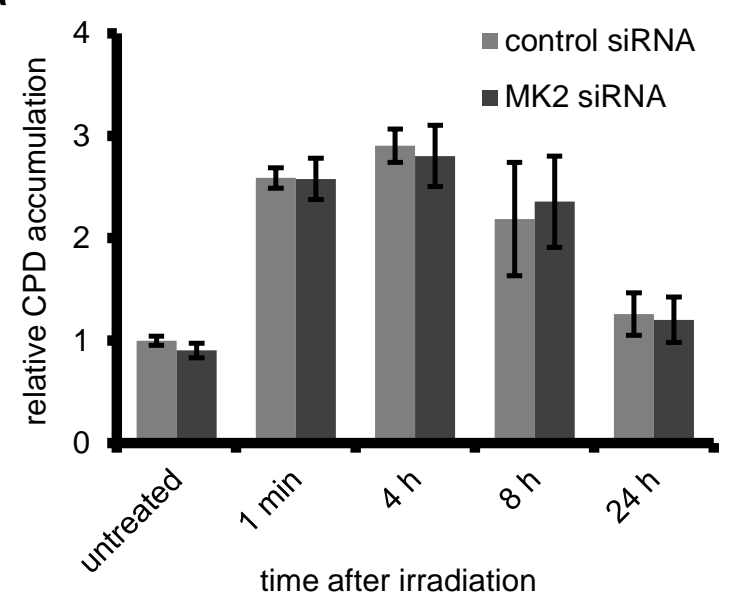

b

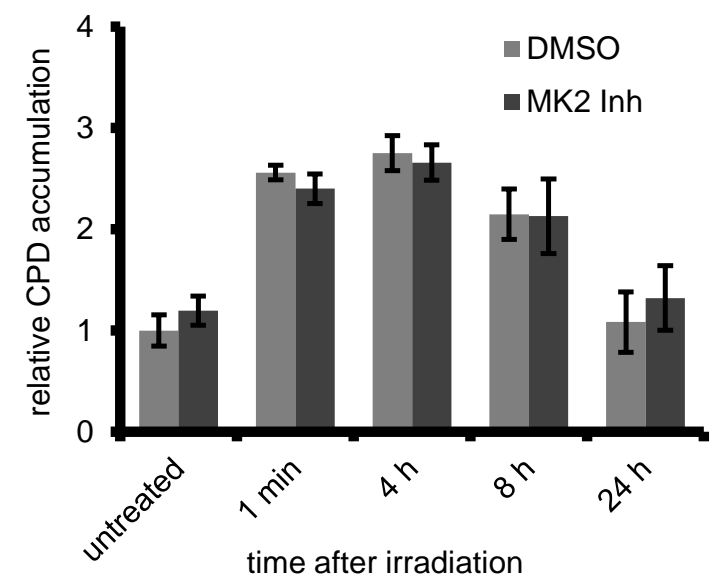

Figure V.2. Accumulation and removal of CPD adducts upon UV irradiation is independent of MK2.

(a) Cells were depleted of MK2 by siRNA-mediated knockdown. After $48 \mathrm{~h}$, cells were irradiated with $30 \mathrm{~J} / \mathrm{m}^{2} \mathrm{UV}-\mathrm{C}$, fixed at indicated time points post irradiation, stained for immunofluorescence analysis and CPD fluorescence intensity was quantified. Results were normalized to the untreated control transfection and corrected for background fluorescence. (b) Cells were treated with MK2 Inh or DMSO for $2 \mathrm{~h}$, then irradiated with $30 \mathrm{~J} / \mathrm{m}^{2} \mathrm{UV}-\mathrm{C}$, fixed at indicated time points post irradiation, stained and analyzed as in $\mathbf{a}$. Conducted by Cathrin Bierwirth. 
However, survival assays using cultured UV-irradiated cells do not necessarily reflect the in vivo situation. Also, knockdown efficiencies can be ambiguous. We therefore made use of mice with genetic ablation of MK2 and its relative MK3 as a genetically defined in vivo model. As detailed previously, MK2 is highly conserved from mouse to human. MK2 and MK3 are closely related and it has been proposed that MK3 can compensate for the loss of MK2 in MK2 knockout mice (Ronkina et al., 2007), making a double knockout of MK2 and MK3 preferable. Here, we assessed the consequences of DNA damage in the skin of mice as a naturally exposed tissue. The backs of MK2/MK3 double knockout (MK2/MK3 DKO) and wild type (WT) animals were irradiated with UV-B light or left untreated. $24 \mathrm{~h}$ post irradiation, the mice were sacrificed and skin sections were prepared and processed for microscopy. The skin sections were subjected to TUNEL staining to detect apoptotic keratinocytes. Figure V.5a shows representative images of skin sections from MK2/MK3 DKO and WT mice, both untreated and exposed to UV irradiation. While samples from UV-irradiated WT animals displayed strong TUNEL-staining, hardly any TUNEL-positive cells were detected in samples from UV-irradiated MK2/MK3 DKO animals. When calculating the number of apoptotic keratinocytes per $\mathrm{mm}$ epidermis for the entire skin samples from all mice, MK2/MK3 DKO mice showed highly significantly less apoptosis in skin after UV exposure compared to WT animals (Figure V.5), demonstrating that MK2 is required for UV-induced cell death in vivo.

a

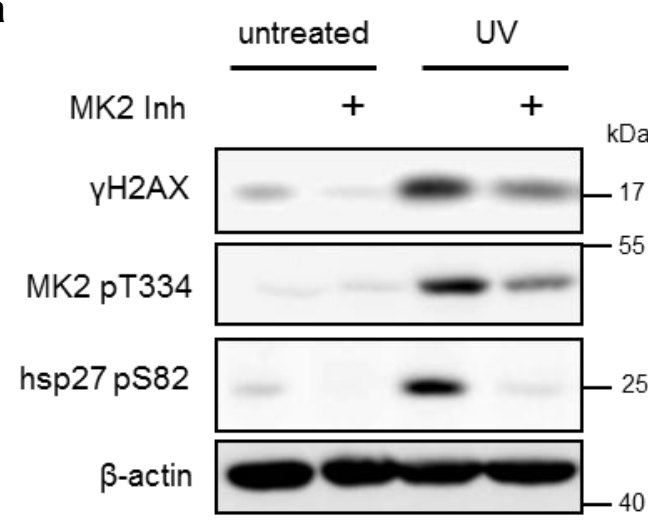

Figure V.3. MK2 inhibition and overexpression of a kinase-dead MK2 impair UV-induced H2AX phosphorylation in keratinocytes.

(a) NHEK cells were pre-incubated with MK2 Inh or DMSO for $4 \mathrm{~h}$, then irradiated with $1,900 \mathrm{~J} / \mathrm{m}^{2}$ UV-B and harvested after $45 \mathrm{~min}$. Cell lysates were analyzed by immunoblotting. Conducted with Cathrin Bierwirth. (b) NHEK cells were transfected with Myc-MK2 WT or a Myc-MK2 kinases dead (KD) construct, exposed to 2,000 $\mathrm{J} / \mathrm{m}^{2}$ UV-B and harvested $45 \mathrm{~min}$ post irradiation. Cell lysates were analyzed by immunoblotting.

b

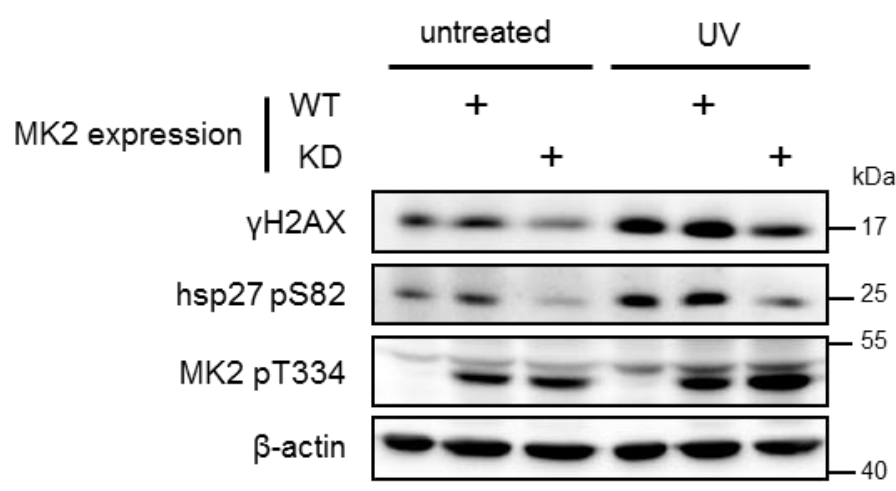




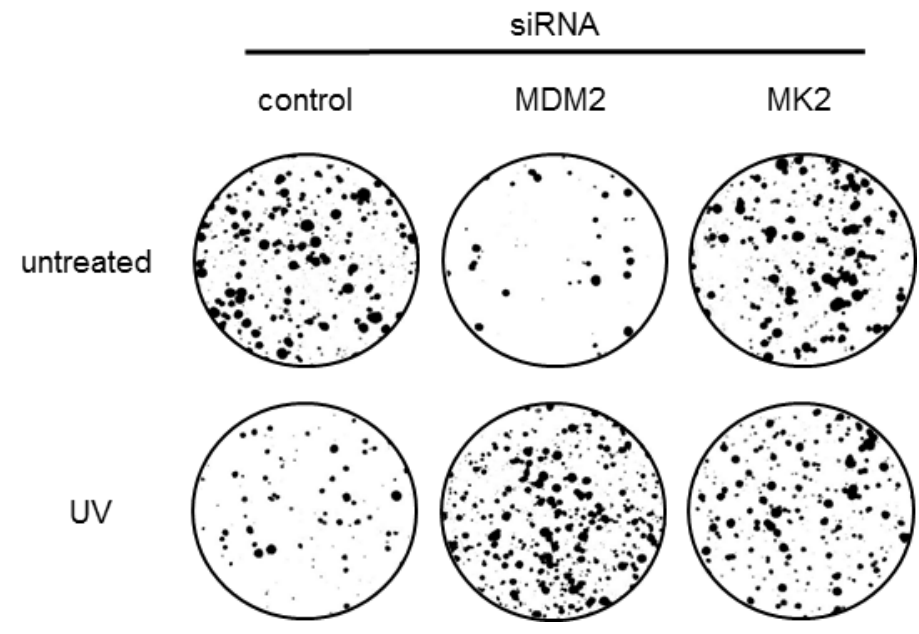

Figure V.4. MK2 depletion reduces UV-induced cell death.

MK2 depletion improves cell survival after UV irradiation. Cells were depleted of Mdm2 or MK2 by siRNA-mediated knockdown. $48 \mathrm{~h}$ later, cells were irradiated with $20 \mathrm{~J} / \mathrm{m}^{2}$ UV-C, reseeded and cell survival analyzed by clonogenic assay. Conducted by Cathrin Bierwirth.

a

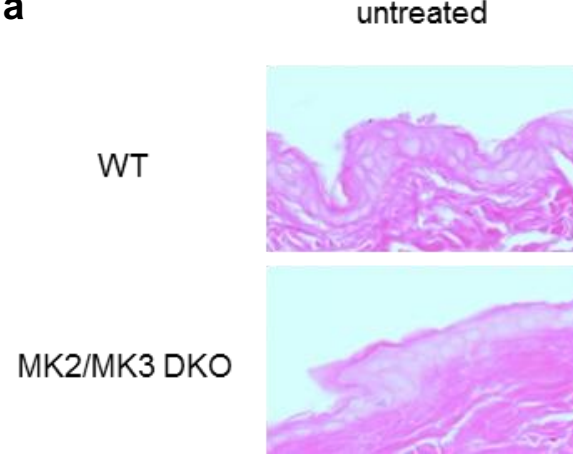

UV

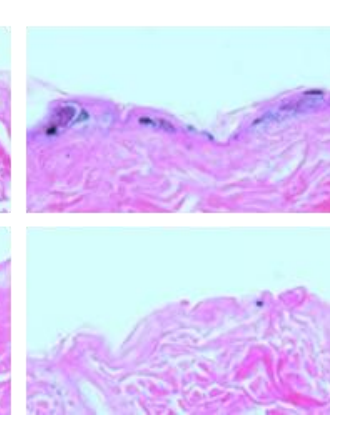

b

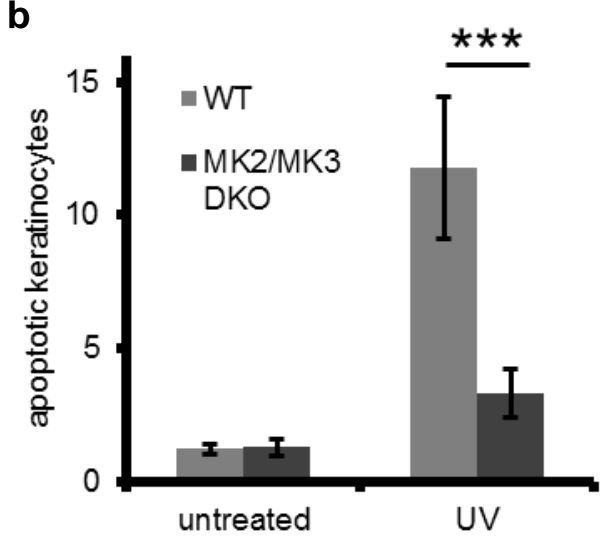

Figure V.5. MK2/MK3 knockout-mice display reduced apoptosis in skin after UV exposure.

(a) Backs of MK2/MK3 knockout-mice (MK2/MK3 DKO) $(n=6)$ and wild type (WT) animals $(n=5)$ were depilated and exposed to $250 \mathrm{~mJ} / \mathrm{m}^{2}$ UV-B irradiation. Areas of $2 \mathrm{~cm}^{2}$ each on the backs of the MK2/MK3 DKO mice were protected from irradiation using a lightproof aluminium cover. In addition, 5 WT mice were not irradiated. Mice were euthanized $24 \mathrm{~h}$ after UV-B irradiation, and both irradiated and non-irradiated dorsal skin samples were processed for immunohistochemistry. Samples were stained for eosin (red) and TUNEL-positive cells (blue). Representative images are shown. (b) Quantification of TUNEL-positive cells per $\mathrm{mm}$ epidermis from entire tissue sections from animals treated as in a. Conducted with Margarete Schön, Department of Dermatology, Venerology and Allergology, Faculty of Medicine, University of Göttingen. 


\section{V.2 MK2 in the gemcitabine response}

\section{V.2.1 MK2 is a determinant of gemcitabine sensitivity}

UV irradiation exhibits its cytotoxic potential by interfering with various cellular processes. The biggest challenge for cells, however, is arguably formed by the UV-induced DNA lesions that interfere with DNA replication in S-phase (Herrlich et al., 2008). We accordingly resolved to test whether the protective effect of MK2 depletion and inhibition is due to a function of MK2 in S-phase. Therefore, we substituted UV irradiation with the nucleoside analogue gemcitabine that causes severe replicative stress as it induces chain termination upon incorporation into DNA and depletes the cellular dNTP pool. Treatment of U2OS cells with gemcitabine induces a strong accumulation of $\mathrm{yH} 2 \mathrm{AX}$. To address the question whether this response also depends on MK2, we performed an RNA interference (RNAi) experiment analogous to the screen: Cells were depleted of MK2 by siRNAmediated knockdown and treated with gemcitabine. Then, H2AX phosphorylation was quantified by immunofluorescence (Figure V.6a). As an alternative to interfere with MK2 activity, we replaced the siRNA with MK2 Inh in a second experiment (Figure V.6b). Both MK2 knockdown and inhibition reduced the $\mathrm{yH} 2 \mathrm{AX}$ levels in gemcitabine-treated cells. We obtained the same result when analyzing $\mathrm{yH} 2 \mathrm{AX}$ accumulation by immunoblotting (Figure V.6c). Furthermore, we found that phosphorylation of Jun N-terminal kinases (JNK) 1 and 2 following gemcitabine treatment is strongly reduced in cells depleted of MK2 (Figure V.6d). These results support the notion of MK2 as a central regulator of the DDR, its depletion not only interfering with gemcitabine-induced H2AX phosphorylation, but also reducing cellular stress signaling.

We thereupon tested whether the changes in gemcitabine-induced $\mathrm{yH} 2 \mathrm{AX}$ levels were reflected by different cell viability and performed a cell proliferation assay. Cells were treated with gemcitabine or left untreated, in the presence or absence of MK2 Inh, for 24 h. Then, the drugs were washed away and changes in cell confluence were analyzed by brightfield microscopy every $24 \mathrm{~h}$ for 11 days (Figure V.7). While cells treated with gemcitabine alone already showed reduced proliferation after 2 days and finally died, cells treated with gemcitabine in the presence of MK2 Inh merely underwent an initial growth delay (days 2-4) but then displayed a proliferation rate comparable to the untreated controls.

Thus, $\mathrm{H} 2 \mathrm{AX}$ phosphorylation as well as the induction of cell death upon gemcitabine treatment appear to depend on MK2. These results are in agreement with our observations made with UV-induced DNA damage. Additionally, as gemcitabine specifically affects DNA replication, this data points to a role of MK2 in this process. 
a

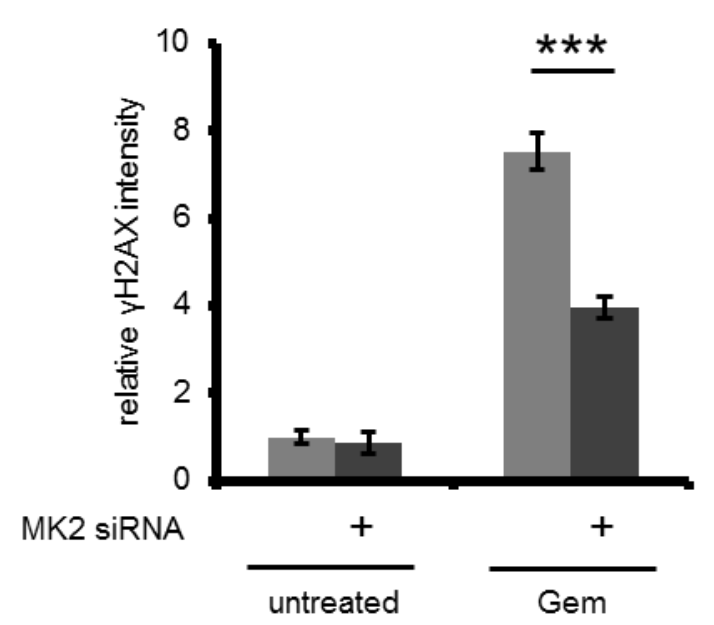

b

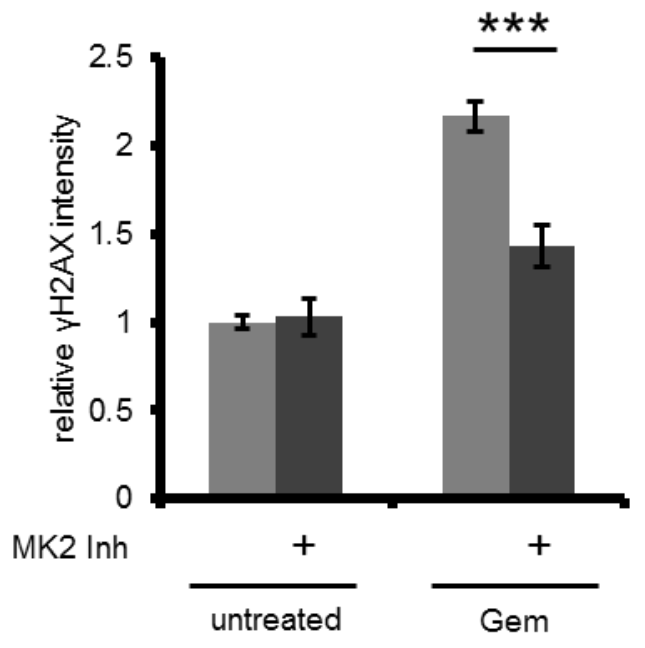

c

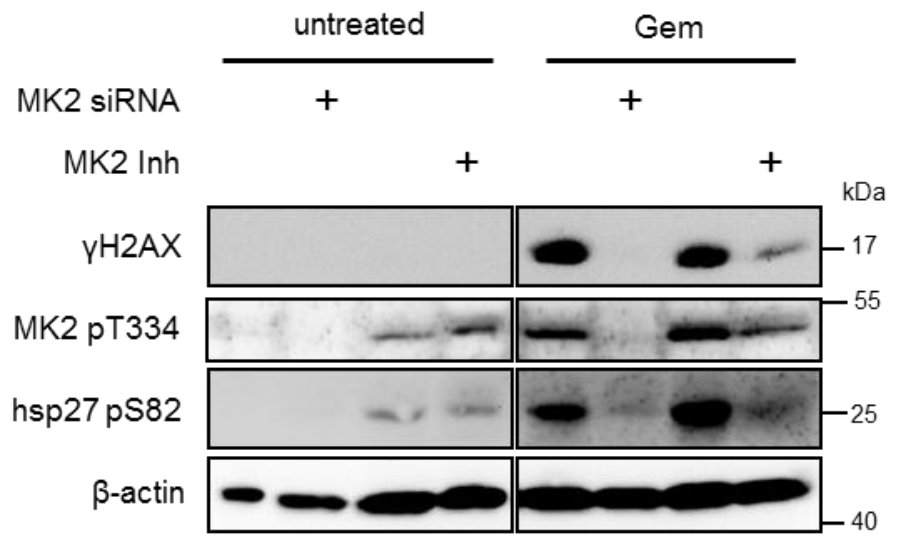

d

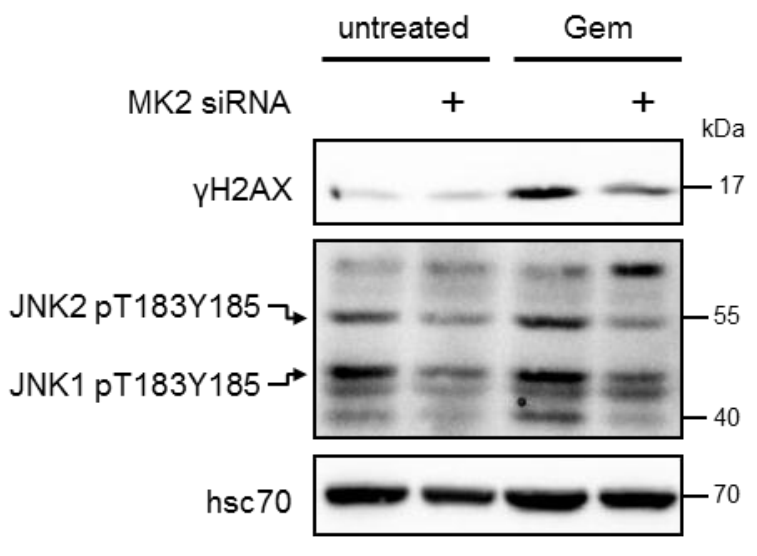

Figure V.6. Gemcitabine-induced H2AX phosphorylation and stress signaling depends on MK2.

(a) Cells were depleted of MK2 by siRNA-mediated knockdown. Thereafter, cells were treated with $100 \mathrm{nM}$ gemcitabine for $20 \mathrm{~h}$ or left untreated. Cells were fixed, stained for immunofluorescence analysis and $\mathrm{yH} 2 \mathrm{AX}$ fluorescence intensity was quantified. Results were normalized to the untreated control transfection and corrected for background fluorescence. (b) Cells were

treated with $200 \mathrm{nM}$ gemcitabine for $24 \mathrm{~h}$ or left untreated and simultaneously treated with MK2 Inh or DMSO. Cells were fixed, stained and analyzed for $\mathrm{yH} 2 \mathrm{AX}$ accumulation as detailed in a. (c) Cells were depleted of MK2 by siRNA-mediated knockdown or left untreated. Thereafter, cells previously transfected with siRNA were treated with $100 \mathrm{nM}$ gemcitabine for $24 \mathrm{~h}$. Previously untreated cells were treated with $100 \mathrm{nM}$ gemcitabine and MK2 Inh or DMSO for $24 \mathrm{~h}$. Cells were harvested and lysates were analyzed by immunoblotting. (d) Cells were depleted of MK2 by siRNA-mediated knockdown and treated as in a. Cells were harvested and lysates were analyzed by immunoblotting. 


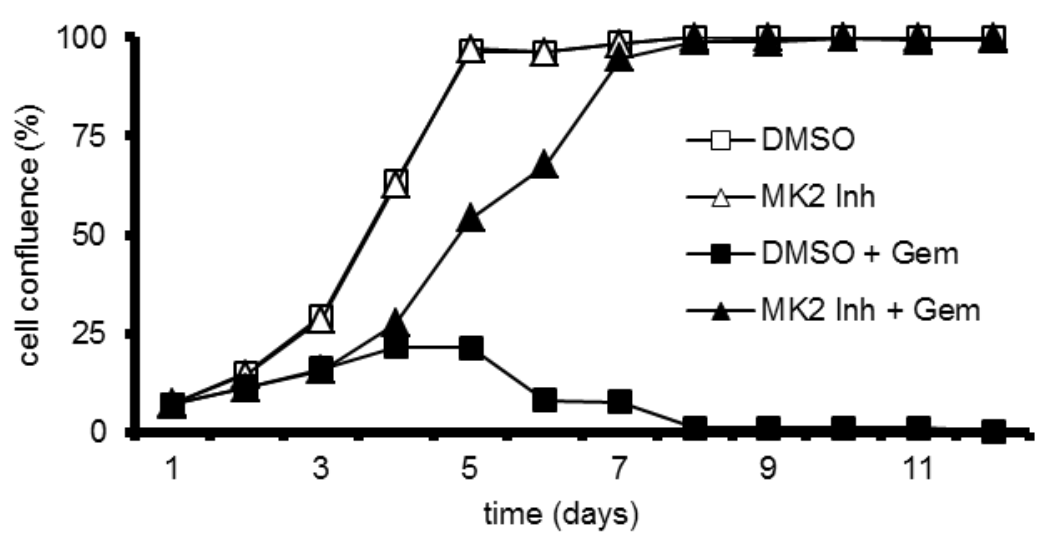

Figure V.7. Gemcitabine-induced cell death depends on MK2.

Cell survival after gemcitabine treatment is improved by MK2 inhibition. On day 1, cells were treated with $100 \mathrm{nM}$ gemcitabine and MK2 Inh or DMSO for $24 \mathrm{~h}$. Cell confluence was determined once a day for 12 days by light microscopy to assess proliferation. Data points represent average of three replicates. Conducted with Cathrin Bierwirth.

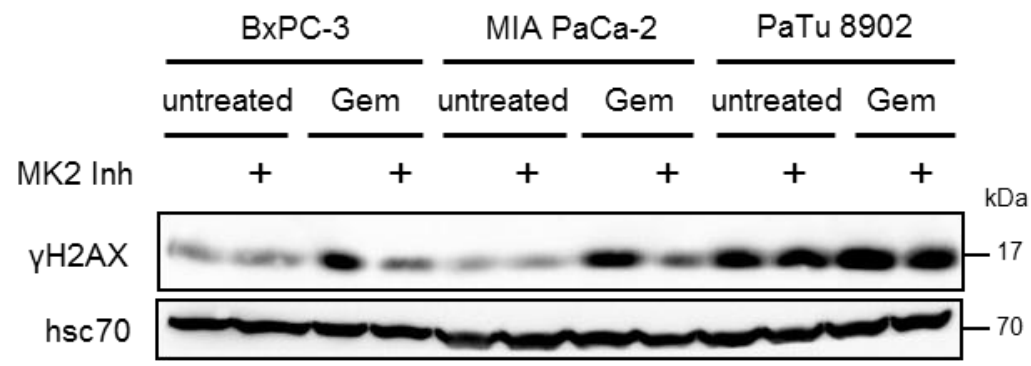

Figure V.8. Gemcitabine-induced H2AX phosphorylation is reduced by MK2 inhibition in gemcitabine-sensitive pancreatic carcinoma cell lines.

Cells were treated with $100 \mathrm{nM}$ gemcitabine or left untreated and simultaneously treated with MK2 Inh or DMSO for $24 \mathrm{~h}$. Thereafter, cells were harvested and lysates were analyzed by immunoblotting.

\section{V.2.2 MK2 is required for the DDR in gemcitabine-sensitive pancreatic carcinoma cell lines}

In the clinics, gemcitabine is applied in the first-line treatment of pancreatic adenocarcinomas. We were therefore interested to see whether H2AX phosphorylation and cell survival following gemcitabine treatment also depend on MK2 in cell lines derived from pancreatic carcinomas. To this end, the three cell lines BxPC-3, MIA PaCa-2 and PaTu 8902 were used. Upon treatment with gemcitabine, we found that BxPC-3 and MIA $\mathrm{PaCa}-2$, both known to be gemcitabine-sensitive (Fryer et al., 2011), displayed a strong 

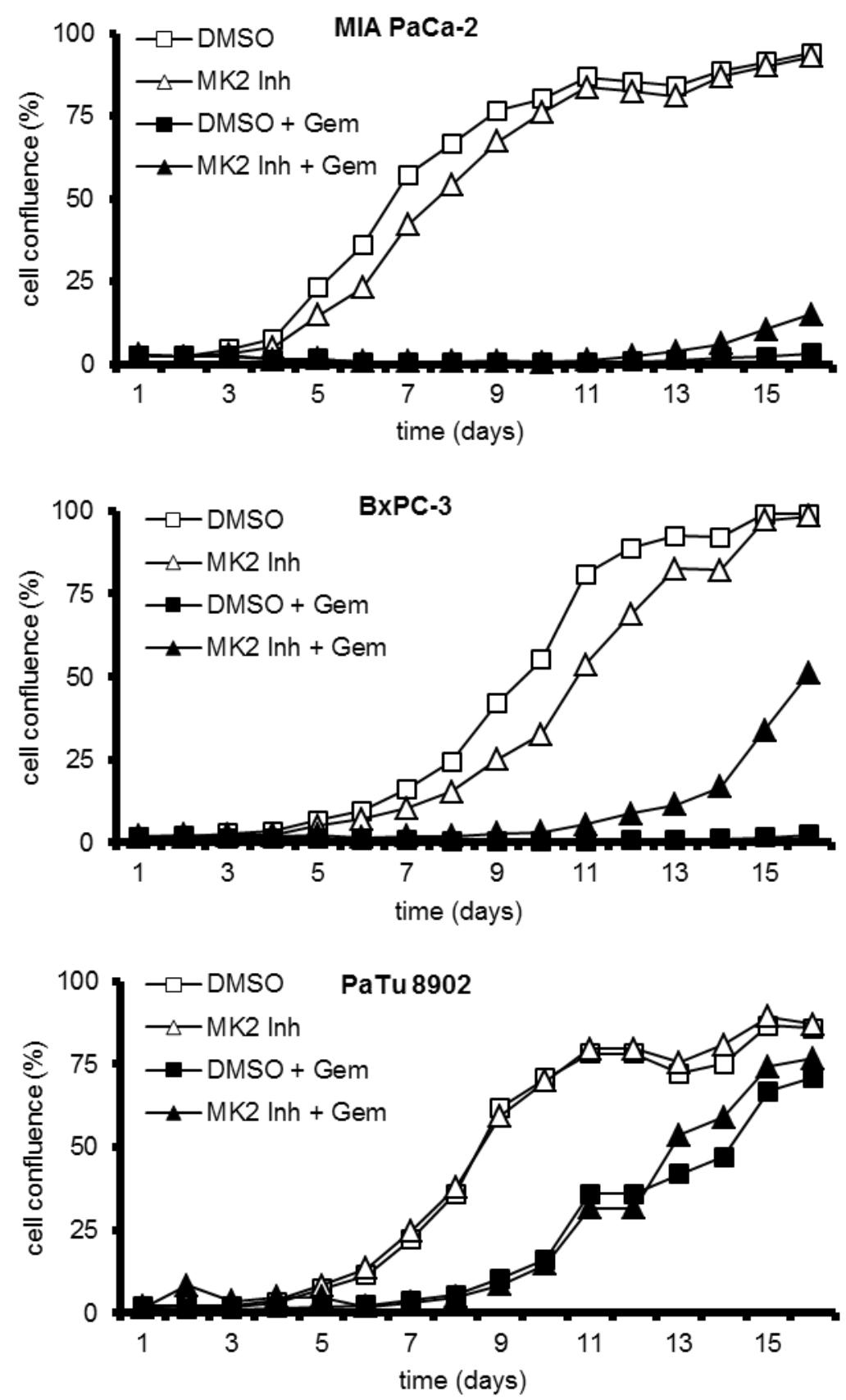

Figure V.9. Gemcitabine-induced cell death is reduced by MK2 inhibition in gemcitabinesensitive pancreatic carcinoma cell lines.

On day 1, cells were treated with $200 \mathrm{nM}$ gemcitabine and MK2 Inh or DMSO for $24 \mathrm{~h}$. Cell confluence was determined once a day for 12 days by light microscopy to assess proliferation. Data points represent average of three replicates.

dependence of $\mathrm{H} 2 \mathrm{AX}$ phosphorylation on MK2 activity: In the presence of MK2 Inh, the gemcitabine-induced increase in $\mathrm{yH} 2 \mathrm{AX}$ levels was strongly reduced (Figure V.8). In the gemcitabine-insensitive PaTu8902 cells yH2AX levels did not change upon exposure to gemcitabine and accordingly MK2 inhibition did not have any effect. We next tested whether the differential $\mathrm{H} 2 \mathrm{AX}$ phosphorylation in response to gemcitabine also translates into differences in their proliferative capacity upon exposure to gemcitabine and MK2 Inh 
(Figure V.9). None of the cell lines showed a significant change in proliferation upon treatment with MK2 Inh alone. Treatment with gemcitabine alone resulted in extensive death of cells in BxPC-3 and MIA PaCa-2 and the cells did not recover. PaTu-8902, on the other hand, displayed a moderate but significant growth delay following gemcitabine exposure, and this was unaffected by MK2 inhibition. Strikingly, BxPC-3 and MIA PaCa-2 cells co-treated with gemcitabine and MK2 Inh started to recover and again proliferate ten to twelve days post treatment.

These results are in line with our observations made with U2OS cells and demonstrate that in cell lines derived from pancreatic carcinomas the sensitivity towards gemcitabine depends on MK2, as well. In agreement with this finding, the gemcitabine-insensitive cell line PaTu-8902 did not display any dependence on MK2.

\section{V.2.3 MK2 acts during DNA replication}

It was previously reported that a reduced responsiveness of cells towards gemcitabine can be due to the induction of a G1 arrest by activation of p53 (Kranz and Dobbelstein, 2006). Furthermore, recent publications suggest that MK2 acts as a regulator of the cell cycle and that its activation is required for the maintenance of the G2/M checkpoint (Manke et al., 2005; Reinhardt et al., 2010). It therefore appeared possible that the protective effect of MK2 depletion and inhibition is due to changes in cell cycle regulation. Hence, we tested whether MK2 inhibition affects the cell cycle distribution of U2OS cells. As a comparison, we treated cells with the pharmacological Mdm2-antagonist Nutlin-3 (Vassilev et al., 2004). While treatment with Nutlin-3 induced a G1 arrest and led to a severe reduction of cells with a DNA content corresponding to S-phase, cells treated with MK2 Inh did not show differences in their cell cycle profile when compared to the DMSOtreated control (Figure V.10a), suggesting that MK2 does not affect cell cycle progression in unperturbed cells.

Considering the above results, it appeared plausible that MK2 exhibits its effects on the DDR to gemcitabine specifically during DNA replication. We therefore assessed the impact of MK2 inhibition on the DDR in cells that are already in S-phase at the time of treatment with gemcitabine. For this, we pulse-labeled cells with 5-ethynyl-2'-deoxyuridine (EdU) prior to gemcitabine treatment and evaluated H2AX phosphorylation by quantitative immunofluorescence. This allowed us to compare the effect of MK2 Inh on the whole cell population with its impact on cells that are in the process of replicating their DNA (i.e. EdU-positive cells) when treated with gemcitabine. Again, we used Nutlin-3 for comparison, as p53 accumulation should not protect cells that are already in S-phase at 
a

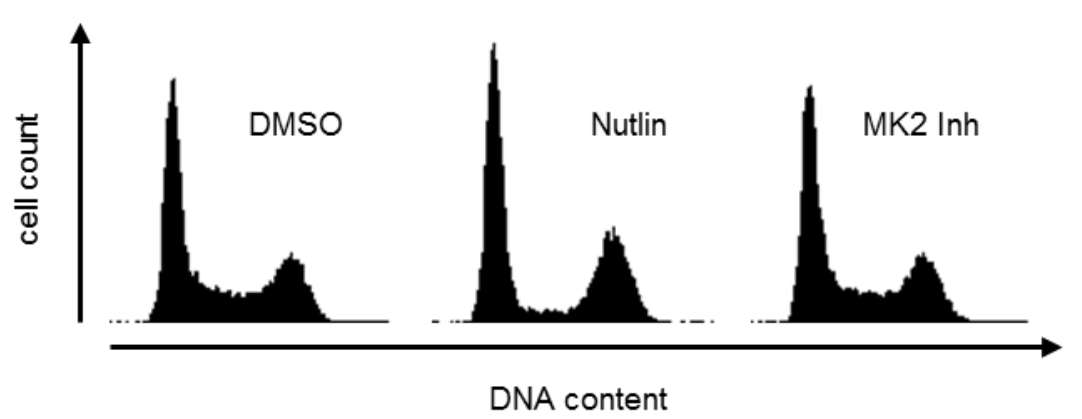

b

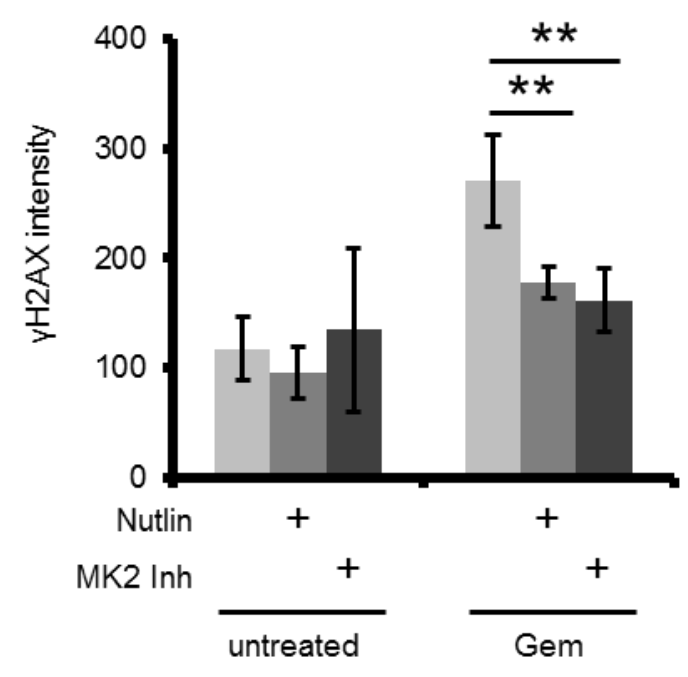

All cells

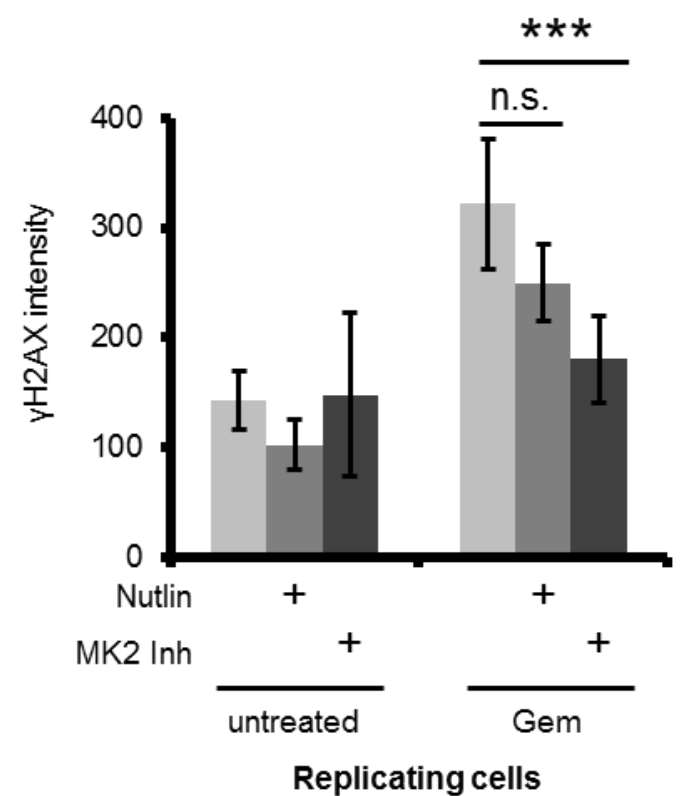

C

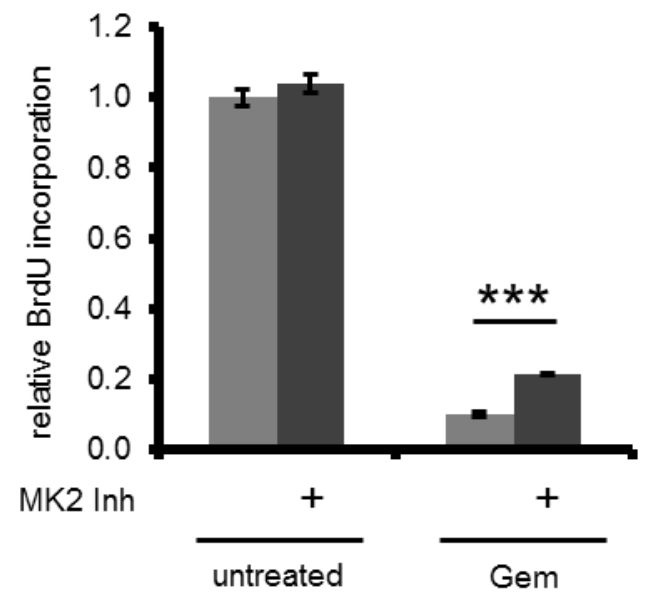

Figure V.10. MK2 inhibition does not affect cell cycle progression but acts during DNA replication upon DNA damage.

(a) MK2 inhibition does not influence cell cycle progression in U2OS. Cells were treated with MK2 Inh, Nutlin-3 or DMSO for $24 \mathrm{~h}$ and cell cycle analysis was performed by flow cytometry. Conducted by Cathrin Bierwirth. (b) MK2 acts in cells during DNA replication. Cells were treated with MK2 Inh, Nutlin-3 or DMSO for $8 \mathrm{~h}$. Following this, replicating cells were labeled by incubation with EdU for $2 \mathrm{~h}$. Afterwards, cells were treated with $300 \mathrm{nM}$ gemcitabine for $22 \mathrm{~h}$, fixed, stained for immunofluorescence analysis and $\mathrm{yH} 2 \mathrm{AX}$ fluorescence intensity was quantified.

The average fluorescence resulting from $\mathrm{YH} 2 \mathrm{AX}$ staining was determined per nucleus and averaged over all nuclei $(n=4)$. To identify replicating cells, nuclei were gated for EdU fluorescence. The average fluorescence resulting from $\mathrm{yH} 2 \mathrm{AX}$ staining was determined for these nuclei only $(n=4)$. Conducted with Priyanka Saini. (c) MK2 inhibition improves slow DNA replication upon gemcitabine treatment. Cells were treated with $100 \mathrm{nM}$ gemcitabine and MK2 Inh or DMSO for $16 \mathrm{~h}$. During the last two hours, cells were labeled with BrdU, then fixed, stained for immunofluorescence analysis and BrdU fluorescence intensity was quantified. Hoechst signal was used to identify cell nuclei. Results were normalized to the untreated control and corrected for background fluorescence. Conducted with Cathrin Bierwirth. 
the time of treatment. As expected, both MK2 Inh as well as Nutlin-3 reduced gemcitabine-induced $\mathrm{yH} 2 \mathrm{AX}$ accumulation when analyzing the whole cell population (Figure V.10b). When the population was gated for EdU-positive cells, however, Nutlin-3 failed to protect the cells to a significant degree but MK2 inhibition still strongly reduced $\mathrm{yH} 2 \mathrm{AX}$ accumulation. Hence, it can be concluded that, while p53 activation reduces the gemcitabine response by arresting cells outside S-phase, inhibition of MK2 impairs the response among those cells that are replicating their DNA.

We hypothesized that the protective effect of MK2 depletion and inhibition upon treatment with gemcitabine was likely to result from an effect of MK2 on DNA replication. To follow up on this idea, we assessed the rate of DNA replication after gemcitabine treatment in dependence of MK2 inhibition by immunofluorescent quantification of 5-bromo-2'deoxyuridine (BrdU) incorporation. Gemcitabine reduced the amount of incorporated BrdU to less than $10 \%$ when compared to untreated cells (Figure V.10c), reflecting a severe block of replication. MK2 inhibition induced a slight increase in the rate of DNA replication, bringing it to about $20 \%$ of the original level, showing that MK2 inhibition can indeed improve the rate of DNA replication upon genotoxic stress. These findings motivated us to search for specific functions of MK2 in DNA replication.

\section{V.2.4 MK2 activity slows down DNA replication in response to gemcitabine while enhancing origin firing}

Replication stress caused by either endogenous or exogenous factors manifests itself as a decrease in the speed of replication forks (Branzei and Foiani, 2010). At the same time, dormant origins that would otherwise be replicated passively are stochastically fired, resulting in an overall increase in the rate of origin firing (Ge et al., 2007; Ibarra et al., 2008).

We used DNA fiber assays (Jackson and Pombo, 1998) to assess the role of MK2 in the regulation of both replication fork speed and origin firing in response to gemcitabine treatment. For DNA fiber analysis, replication forks were labeled by sequential incorporation of the nucleoside analogues 5-Chloro-2'-deoxyuridine (CldU) and 5-lodo-2'deoxyuridine (IdU). The DNA was then spread on glass slides and the incorporated nucleosides were stained with fluorescent antibodies to visualize replicating structures. These were imaged by confocal microscopy and analyzed. Figure IV.1 provides an overview of the resulting structures. For the analysis of changes in replication fork speed, the lengths of the CldU- and IdU-labeled tracks of on-going, unidirectional forks were measured. Origin firing was assessed by quantification of newly fired origins during the 
a

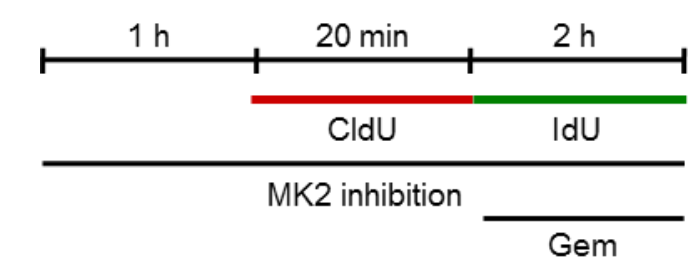

C
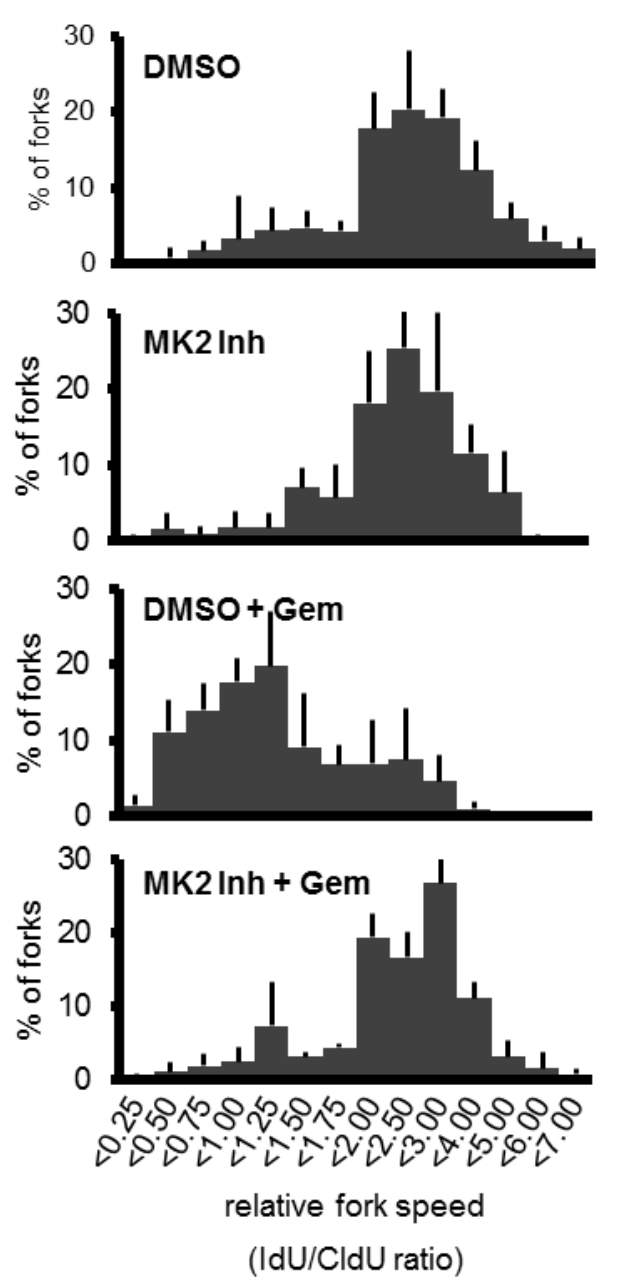

b

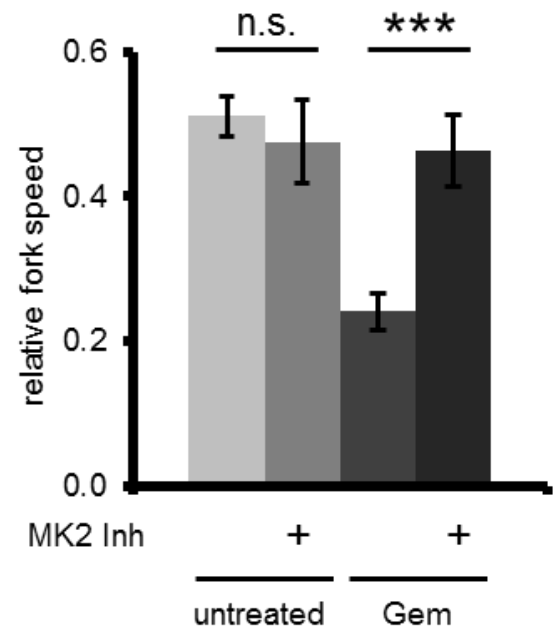

replication fork speed

Figure V.11. Reduction of replication fork speed caused by gemcitabine is rescued by MK2 inhibition.

(a) Labeling protocol for DNA fiber analysis of replication fork speed. U2OS cells were pretreated with MK2 Inh or DMSO for $1 \mathrm{~h}$. Treatment with MK2 Inh or DMSO was continued throughout the experiment. Cells were then pulse labeled with CldU for $20 \mathrm{~min}$. Afterwards, cells were pulse labeled with IdU for $2 \mathrm{~h}$ and simultaneously exposed to $400 \mathrm{nM}$ gemcitabine. CldU and IdU were detected using specific primary antibodies and secondary antibodies in red and green, respectively. (b,c) MK2 inhibition rescues reduced replication fork speed caused by gemcitabine. (b) Average relative replication fork speed (ratio of length of IdU-labeled tracks vs. length of CldU-labeled tracks) in cells treated as in a in dependence of gemcitabine and MK2 inhibition $(n=3)$. (c) Distribution of replication fork speeds in cells treated as in a.

red label (corresponding to on-going, bidirectional forks in Figure IV.1) as a percentage of all red-labeled structures.

Fiber assays are applied to monitor rapid changes in replication. For this reason, siRNAmediated knockdown of the kinases of interest was not convenient. Instead, kinase activity was blocked using pharmacological inhibitors in all fiber assays. It has previously been reported that the depletion of kinases by siRNA for fiber assays yields similar ten- 
a

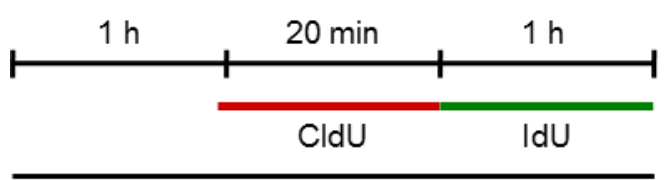

MK2 inhibition

Gem

C

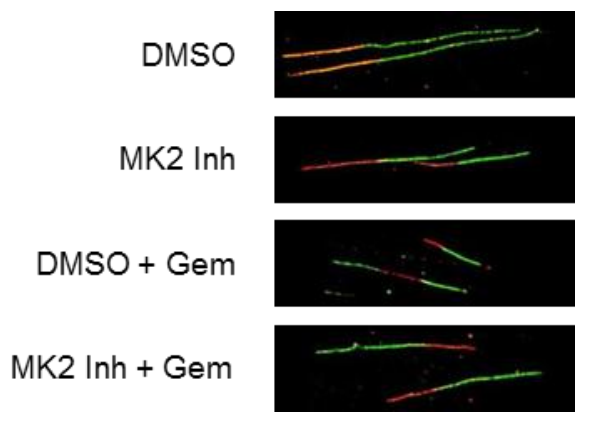

b

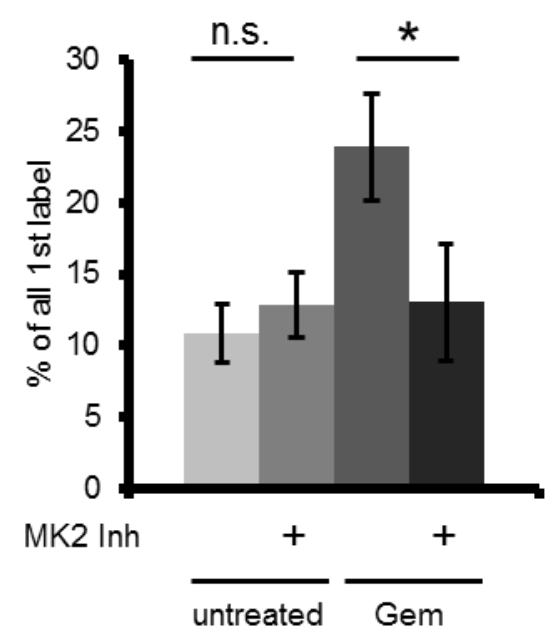

origin firing

d

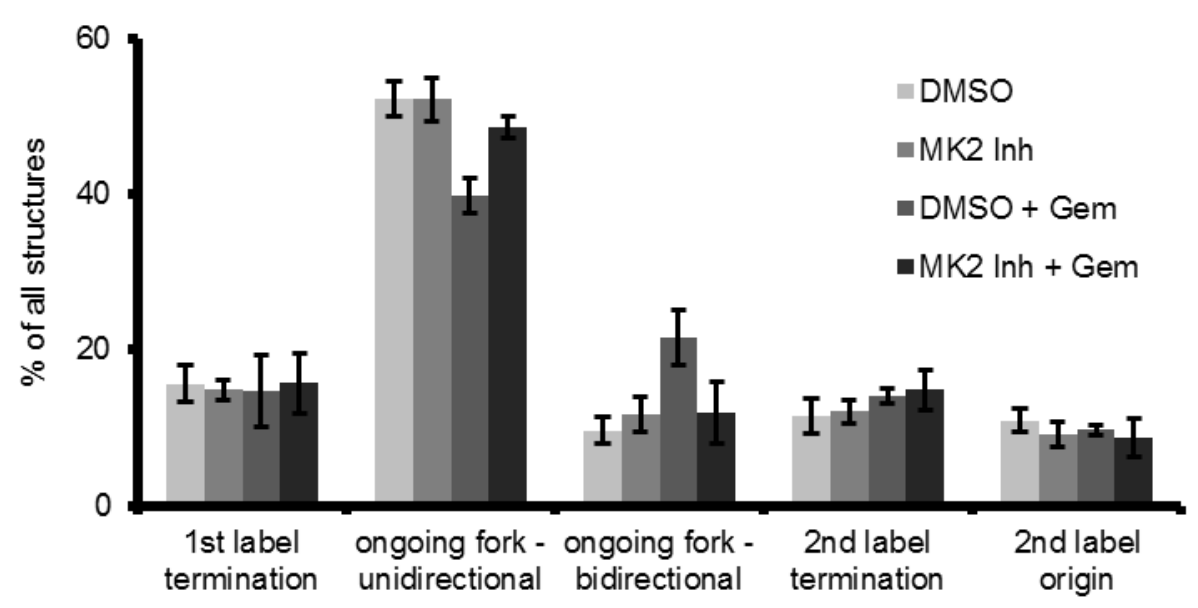

Figure V.12. Increase of origin firing caused by gemcitabine is rescued by MK2 inhibition.

(a) Labeling protocol for DNA fiber analysis of origin firing. U2OS cells were pre-treated with MK2 Inh or DMSO for $1 \mathrm{~h}$, then pulse labeled with CldU and IdU in the presence of $400 \mathrm{nM}$ gemcitabine for $20 \mathrm{~min}$ and $1 \mathrm{~h}$, respectively. CldU and IdU were detected as detailed in Figure V.11. (b) MK2 inhibition rescues increased origin firing caused by gemcitabine. Quantification of origin firing in cells in dependence of gemcitabine and MK2 inhibition. First label origins (green-red-green fibers) are shown as percentage of all red-labeled fibers $(n=3)$. (c) Representative images of fibers from cells treated as in a. (d) Quantification of all structures obtained by DNA fiber analysis as detailed in $\mathbf{a}$.

dencies as the application of corresponding inhibitors, but that these changes are - due to the reason detailed above - less significant (Petermann et al., 2010b).

To investigate the role of MK2 in DNA replication upon gemcitabine exposure, cells were treated with MK2 Inh, then labeled sequentially with CldU and IdU. Gemcitabine was added during the second label (Figure V.11a). As depicted in Figure V.11b, analysis of the resulting tracks revealed that replication fork speed is heavily reduced by gemcitabine 
treatment, which was already suggested by the strong reduction in BrdU incorporation described above. Strikingly, this effect was completely rescued in the presence of MK2 Inh. This change was not only evident in the average fork speed but also immediately visible when evaluating the distribution of fork speeds in histograms (Figure V.11c). Importantly, it should be noted that the effect of gemcitabine on the fork speed most likely results from its incorporation into DNA and not the depletion of dNTP pools since dNTP pools are only affected after prolonged inhibition of RNR (Petermann et al., 2010a).

We next asked whether MK2 inhibition also affects origin firing, which we expected to be increased by gemcitabine treatment due to the firing of dormant origins upon fork stalling. For this, gemcitabine was kept in the culture media during both the CldU as well as the IdU label (Figure V.12a). This was required as origins that are fired during the CldU labeling were analyzed. As assumed, cells exposed to gemcitabine reacted with markedly increased origin firing. Strikingly, this enhancement in origin firing also was rescued by inhibition of MK2 (Figure V.12b, Figure V.12c shows representative images of fibers). The frequency of other replication structures remained largely untouched by MK2 inhibition (Figure V.12d).

In summary, these results revealed that in the presence of replicative stress caused by gemcitabine, MK2 inhibition restores the replication fork speed while it decreases excess origin firing. Thus, the cell is capable of adapting its replication not only in response to the presence of a toxic nucleoside analogue, but also as a function of MK2 activity.

\section{V.3 Interplay of MK2 and Chk1}

\section{V.3.1 Reduction of Chk1 levels leads to an enhanced DNA damage response and cell cycle arrest, but these effects depend on MK2}

DNA replication, even in unperturbed cells, is subject to tight regulation. Upon randomly occurring replication errors, the cell activates an intra-S-phase checkpoint which is mainly mediated by ATR and the checkpoint kinase Chk1 (Feijoo et al., 2001; Heffernan et al., 2002; Miao et al., 2003; Zachos et al., 2003). Chk1 depletion or inhibition leads to the abrogation of the damage-induced S-phase checkpoint (Xiao et al., 2005) and has deleterious effects on cells, ranging from increased replication initiation and the induction of DNA damage signaling to DNA breakage and cell death (Gagou et al., 2010; Syljuasen et al., 2005). Thus, Chk1 inhibition and depletion induce replicative stress in a very similar way as UV lesions and gemcitabine. Our previous results demonstrate that MK2 depletion or inhibition has an opposite effect, protecting cells from the consequences of DNA 


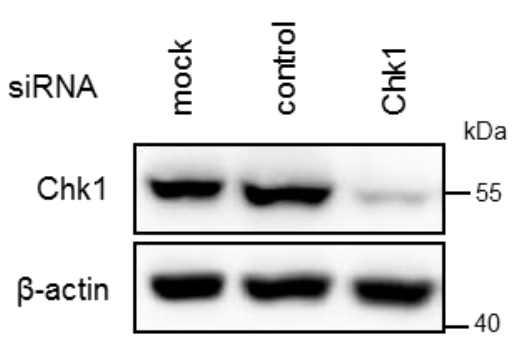

C

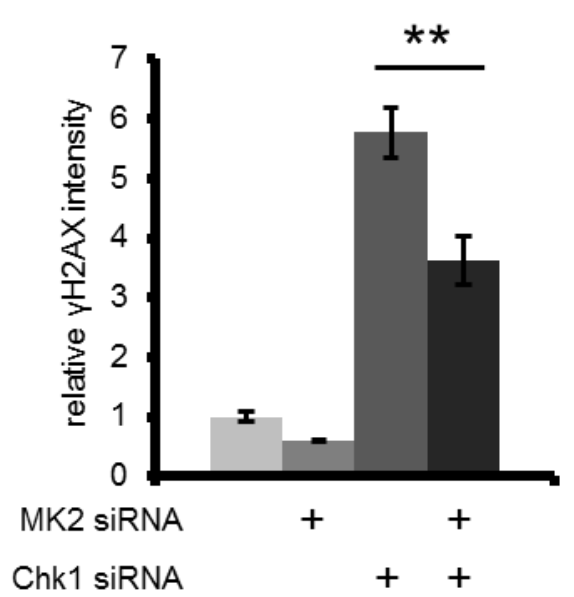

b

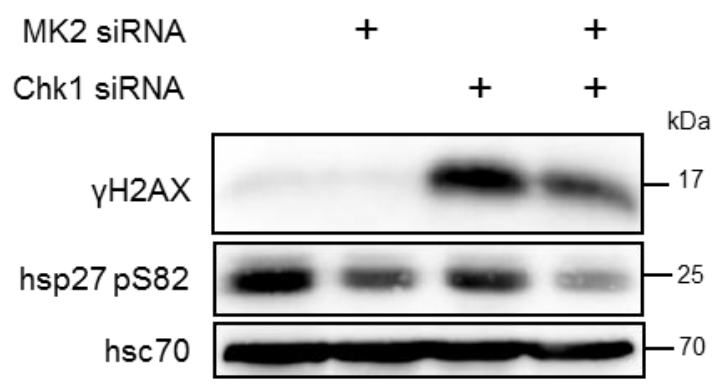

d

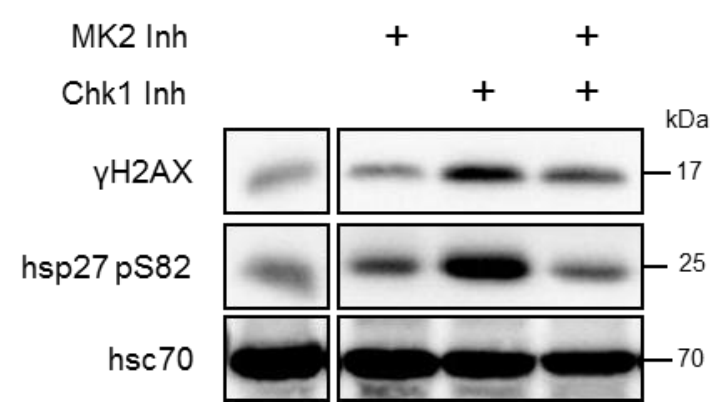

Figure V.13. H2AX phosphorylation upon Chk1 depletion or inhibition depends on MK2.

(a) Depletion efficiencies of MK2 by siRNA-mediated knockdown. Cells were harvested $48 \mathrm{~h}$ after knockdown and protein lysates were analyzed by immunoblotting. (b,c) H2AX phosphorylation upon Chk1 depletion is reduced by co-depletion of MK2. (b) Cells were depleted of MK2 by siRNAmediated knockdown, harvested $48 \mathrm{~h}$ later and cell lysates were analyzed by immunoblotting. (c) Cells were depleted of MK2 and Chk1 by siRNA-mediated knockdown, fixed, stained for immunofluorescence analysis after $48 \mathrm{~h}$ and $\mathrm{yH} 2 \mathrm{AX}$ fluorescence intensity was quantified. Results were normalized to the control transfection and corrected for background fluorescence. (d) MK2 inhibition reduces $\mathrm{H} 2 \mathrm{AX}$ phosphorylation induced by Chk1 inhibition. Cells were treated with MK2 Inh, Chk1 Inh or DMSO for $12 \mathrm{~h}$, then harvested and cell lysates were analyzed by immunoblotting.

damage in S-phase. On the other hand, MK2 and Chk1 have almost identical substrate specificity and it has been suggested that they serve similar functions in the DDR. To shed some light on the relationship between the two kinases, we were interested in testing whether the genotoxic effects of Chk1 inhibition and depletion also depend on MK2.

To this end, we used RNAi to deplete cells of Chk1. Transfection of Chk1 siRNA resulted in a near-complete knockdown of Chk1 on protein level (Figure V.13a). We depleted cells of MK2, Chk1 or both and analyzed the accumulation of $\mathrm{yH} 2 \mathrm{AX}$ by immunoblotting (Figure V.13b) as well as by quantitative immunofluorescence (Figure V.13c). Chk1 knockdown strongly enhanced $\mathrm{H} 2 \mathrm{AX}$ phosphorylation, which is in line with previous ob- 


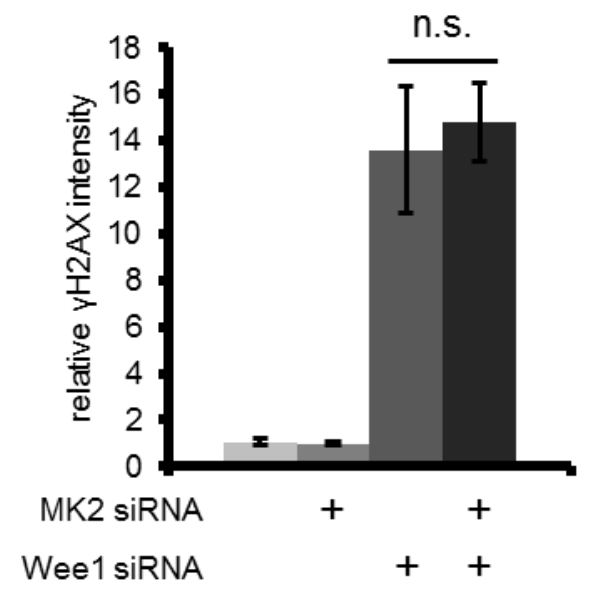

Figure V.14. H2AX phosphorylation upon Wee1 depletion does not depend on MK2. Cells were depleted of MK2 and Wee1 by siRNA-mediated knockdown, fixed, stained for immunofluorescence analysis after $48 \mathrm{~h}$ and YH2AX fluorescence intensity was quantified. Results were normalized to the control transfection and corrected for background fluorescence.

servations (Syljuasen et al., 2005; Xiao et al., 2005). As was the case upon treatment with UV or gemcitabine, MK2 depletion reduced this effect. To confirm the results obtained with knockdown of Chk1, we used a pharmacological inhibitor of Chk1, SB218078 ((Jackson et al., 2000); called Chk1 Inh from here on) and combined it with MK2 Inh. Analysis by immunoblotting revealed that inhibition of Chk1 induced strong H2AX phosphorylation and that MK2 inhibition rescued this effect (Figure V.13d).

\section{V.3.2 Depletion of Wee1 induces genotoxic stress that is independent of MK2}

Depletion of the kinase Wee1 has similar genotoxic effects as Chk1 depletion (Beck et al., 2010). This is due to the fact that both act as negative regulators CDK1 and CDK2. As we found that MK2 depletion alleviates the genotoxic effects caused by Chk1 depletion, a similar effect on the consequences of Wee1 depletion appeared possible. Simultaneous knockdown of Wee1 and MK2, however, did not lead to a reduction of H2AX phosphorylation when compared to Wee1 knockdown alone (Figure V.14). This finding suggests that depletion of Chk1 affects the DDR in a way that is distinct from that of Wee1 depletion and that MK2 is only required for the genotoxic effects of Chk1 depletion.

\section{V.3.3 Depletion of MK2 alleviates the replication block induced by Chk1 depletion and promotes cell survival}

As a consequence of replication fork stalling upon Chk1 depletion or inhibition, it has been observed that cells accumulate in the S-phase of the cell cycle (Forment et al., 2011; Syljuasen et al., 2005). Given the protective effect of MK2 depletion described above, we next used flow cytometry to investigate whether MK2 depletion can also rescue the trapping of Chk1-depleted cells in S-phase. Depletion of Chk1, as expected, resulted in 
an increase in the fraction of cells with a DNA content corresponding to S-phase (Figure V.15a), and this effect was accompanied by reduced EdU incorporation (Figure V.15c), indicating that cells depleted of Chk1 show decreased overall DNA replication. Again,

a

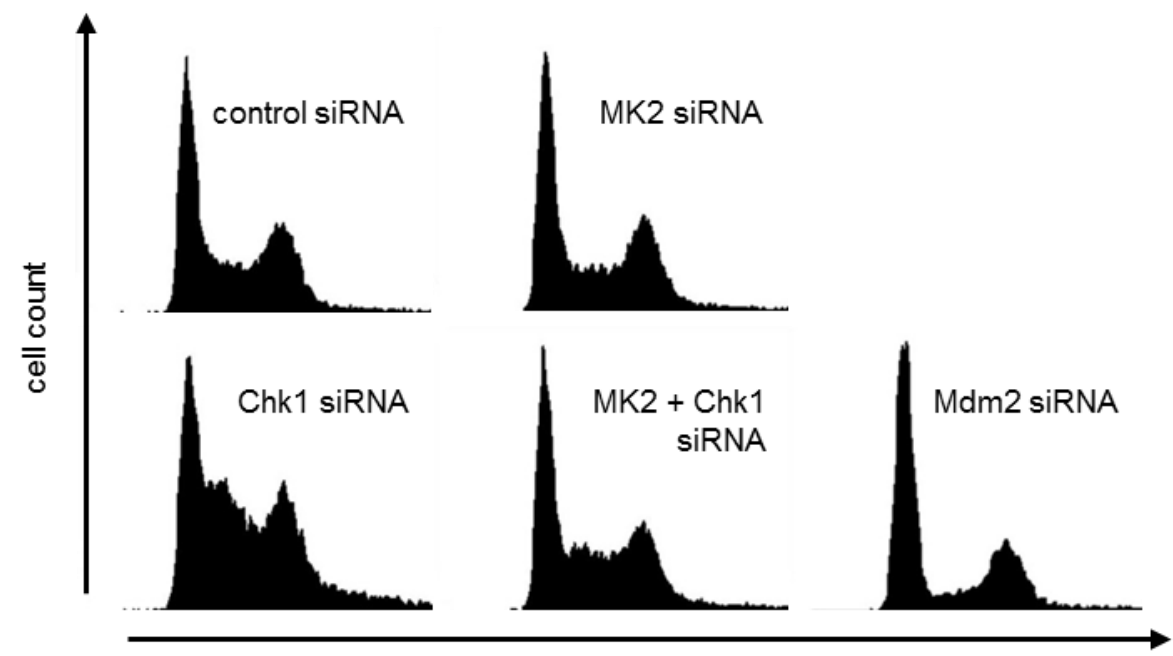

DNA content

b

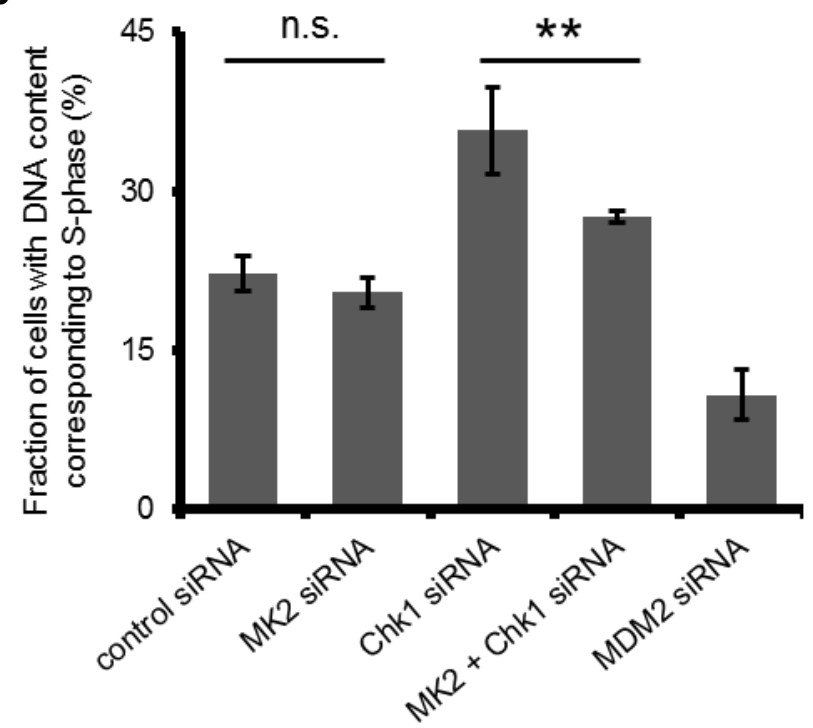

C

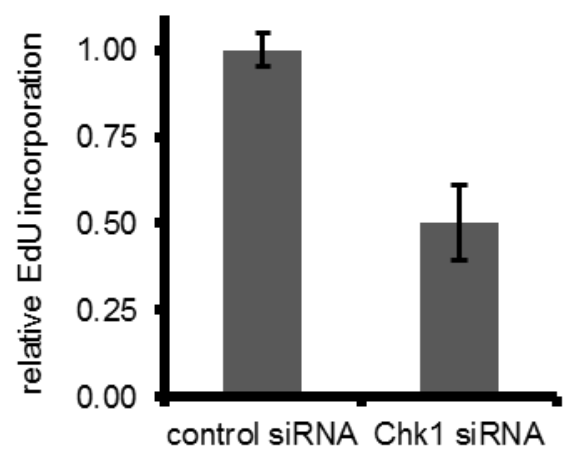

Figure V.15. The replication block induced by Chk1 depletion depends on MK2.

$(\mathbf{a}, \mathbf{b}) \mathrm{S}$-phase arrest of Chk1-depleted cells is rescued by co-depletion of MK2. (a) Cells were depleted of MK2, Chk1 and Mdm2 as a positive e control by siRNA-mediated knockdown and fixed $48 \mathrm{~h}$ later. Cell cycle analysis was performed by flow cytometry. Profiles are representative of four independent experiments. (b) The fraction of cells in different phases of the cell cycle was analyzed from the cell cycle profiles shown in $\mathbf{a}(n=4)$. (c) Chk1 knockdown induces a replication block arrest. Cells were depleted of Chk1 by siRNA-mediated knockdown. $42 \mathrm{~h}$ later, cells were labeled with EdU for $2 \mathrm{~h}$, then fixed, stained for immunofluorescence analysis and EdU fluorescence intensity was quantified as a measure for DNA replication. Results were normalized to the control transfection and corrected for background fluorescence. Conducted with Cathrin Bierwirth. 


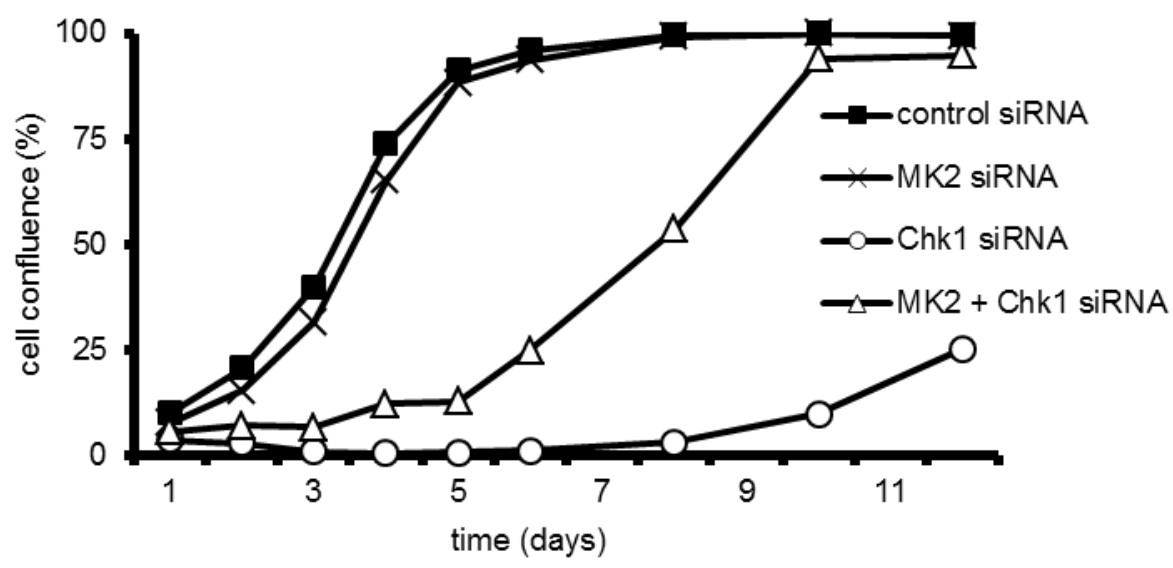

Figure V.16. Cell proliferation after Chk1 depletion is improved by co-depletion of MK2.

Cells were depleted of MK2 and Chk1 by siRNA-mediated knockdown and reseeded for proliferation analysis. $24 \mathrm{~h}$ later, measurement was started (day 1). Cell confluence was measured once a day (days $1-6$ ) or once every two days (days 7-12). Data points represent average of three replicates. Conducted with Cathrin Bierwirth.

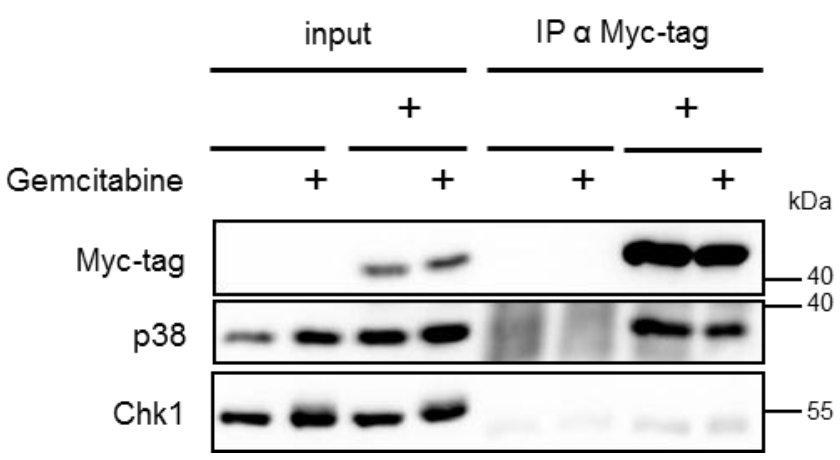

Figure V.17. MK2 does not physically interact with Chk1.

Myc-MK2 WT was ectopically expressed in U2OS cells. For controls, a corresponding empty vector was transfected. Cells were treated with $200 \mathrm{nM}$ gemcitabine for 12 h. Immunoprecipitation using Myc-tag-specific antibodies was performed and precipitated protein was analyzed by immunoblotting.

Mdm2 depletion was used as a control for the activation of the G1 checkpoint. Simultaneous knockdown of MK2 and Chk1 reduced the accumulation of cells in S-phase compared to cells depleted of Chk1 alone (Figure V.15a), and this decrease was significant (Figure V.15b).

Next, we also assessed the potential of MK2 depletion to reverse the proliferation block induced by depletion of Chk1. We found that, while Chk1-depleted cells displayed a strongly reduced proliferation rate and only started to recover about a week after the knockdown, cells co-depleted of both Chk1 and MK2 showed highly improved proliferation potential (Figure V.16). 
Although it has been reported that DNA damage-induced activation of MK2 and Chk1 are independent events (Reinhardt et al., 2007), we considered that one reason for the striking dependence of Chk1 depletion effects on MK2 could be a direct interaction between the two. According to this hypothesis, Chk1 could thus influence the phosphorylation status and thereby conformation or interaction partners of MK2 or its intracellular localization. We therefore tested by ColP whether Chk1 and MK2 physically interact. While the known MK2-interactor p38 precipitated with ectopically overexpressed MK2, Chk1 failed to do so, both in the presence and absence of gemcitabine-induced DNA damage (Figure V.17). While this result suggests that MK2 and Chk1 do not form a stable complex, it cannot be ruled out that Chk1 still directly influences the activity of MK2 as for such a regulation a stable interaction is not required.

In conclusion, we found that MK2 is required for the DDR and the accumulation of cells in S-phase that occur upon elimination of Chk1. Hence, Chk1 is not strictly required for Sphase progression. Rather, MK2 appears to mediate a block in DNA synthesis when Chk1 is absent, but this block does not originate from a stable physical interaction of MK2 and Chk1.

\section{V.3.4 Chk1 inhibition attenuates replication fork progression while enhancing origin firing, and both effects depend on MK2}

To shed more light on the role MK2 plays in DNA replication, we directly assessed the effects of MK2 and Chk1 inhibition by DNA fiber assays. Cells were treated with MK2 Inh and Chk1 Inh, and replication forks were labeled by sequential incorporation of CldU and IdU as before (Figure V.18a). Chk1 inhibition strongly increased origin firing (Figure V.18b) and decreased replication fork speed (Figure V.18c), in line with previous reports (Maya-Mendoza et al., 2007; Petermann et al., 2006; Petermann et al., 2010b). Figure V.18d shows the distribution of replication fork speeds. Representative images of fibers are displayed in Figure V.18e. Simultaneous treatment with MK2 Inh, however, rescued enhanced origin firing almost completely and also improved the fork speed. Again, the frequency of other replication structures remained largely unchanged (Figure V.18f).

Taken together, the above results extend the role of MK2 beyond a restricted function in the response to exogenous DNA damage to it being a central determinant of the cellular response to replicative stress.

\section{V.3.5 Several known regulators of origin firing are unaffected by MK2}

Given the fact that inhibition of MK2 completely rescued the increased origin firing caused by Chk 1 inhibition but only slightly improved slowed fork speed and that Chk1 is known to 


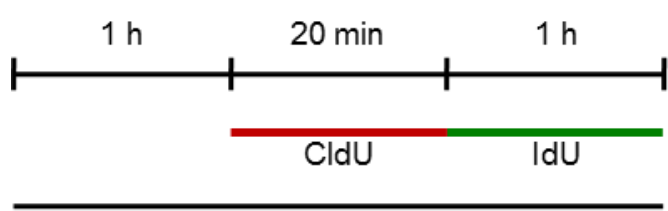

Inhibitor treatment

b

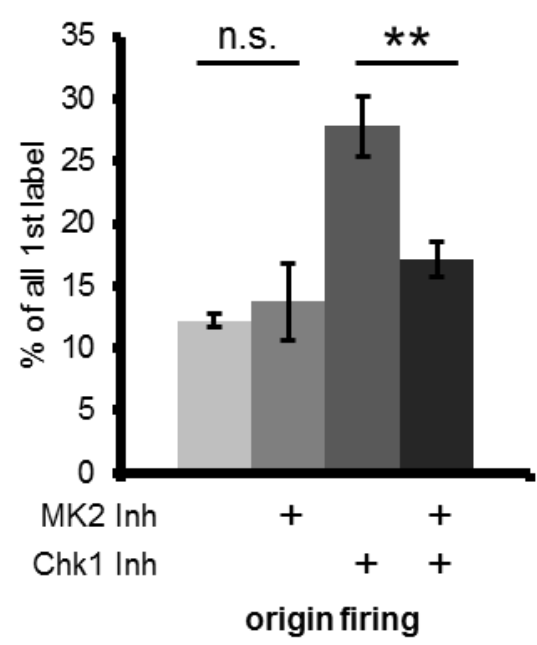

C

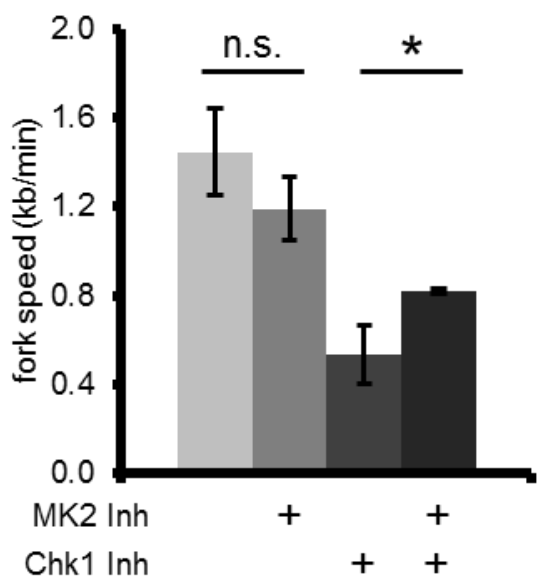

replication fork speed

f

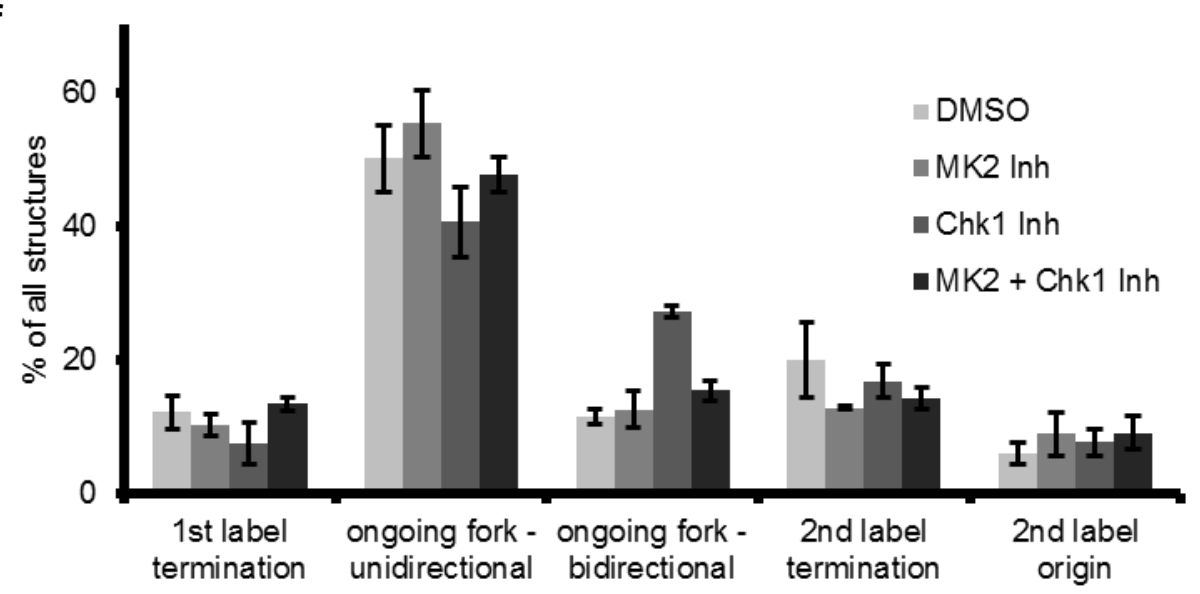

d
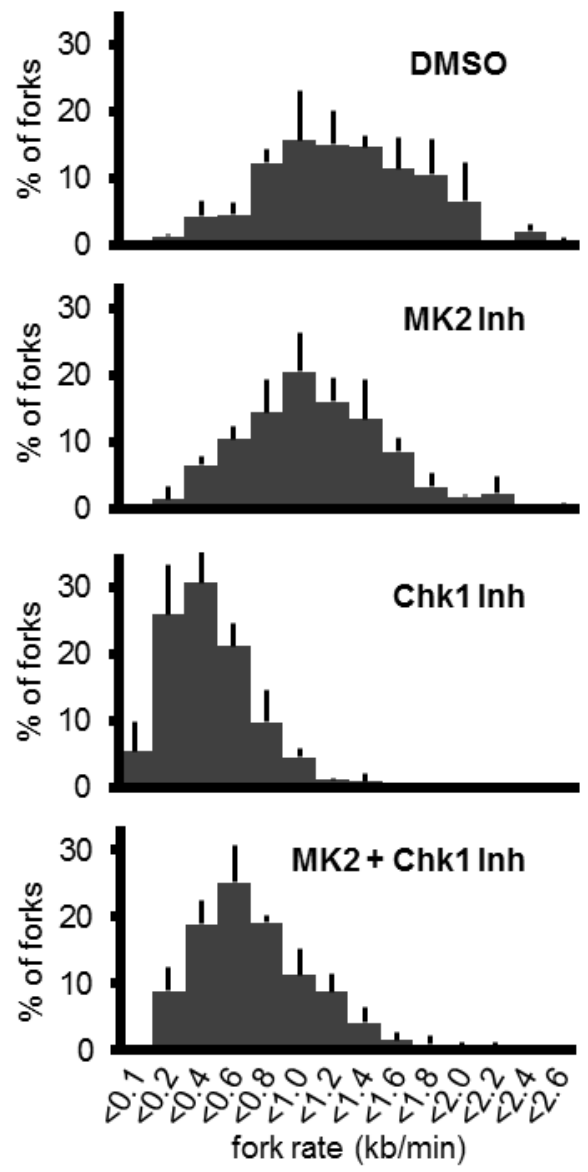

e

DMSO

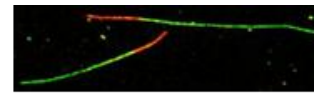

MK2 Inh

Chk1 Inh
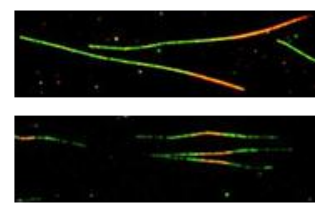

MK2 + Chk1 Inh

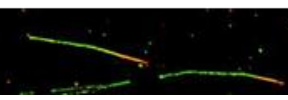

Figure V.18. Reduced replication fork speed and increased origin firing upon Chk1 inhibition depend on MK2.

Legend on next page. 
Legend to figure 18. (a) Labeling protocol for DNA fiber analysis. U2OS cells were pre-treated with MK2 Inh, Chk1 Inh or DMSO for $1 \mathrm{~h}$ and then pulse labeled with CldU for 20 min and IdU for $1 \mathrm{~h}$ in the presence of MK2 Inh, Chk1 Inh or DMSO. CldU and IdU were detected using specific primary antibodies and secondary antibodies in red and green, respectively. (b) MK2 inhibition rescues increased origin firing caused by Chk1 inhibition. Quantification of origin firing in cells in dependence of Chk1 and MK2 inhibition. First label origins (green-red-green fibers) are shown as percentage of all red-labeled fibers $(n=3)$. (c,d) MK2 inhibition improves the reduced replication fork speed following Chk1 inhibition. (c) Average replication fork speed in cells in dependence of Chk1 and MK2 inhibition. The length of CldU tracks of on-going forks was used for calculation of the replication fork speed $(n=3)$. (d) Distribution of replication fork speeds in cells treated as detailed above. (e) Representative images of fibers from cells treated as in a. (f) Quantification of all structures obtained by DNA fiber analysis as detailed in $\mathbf{a}$.

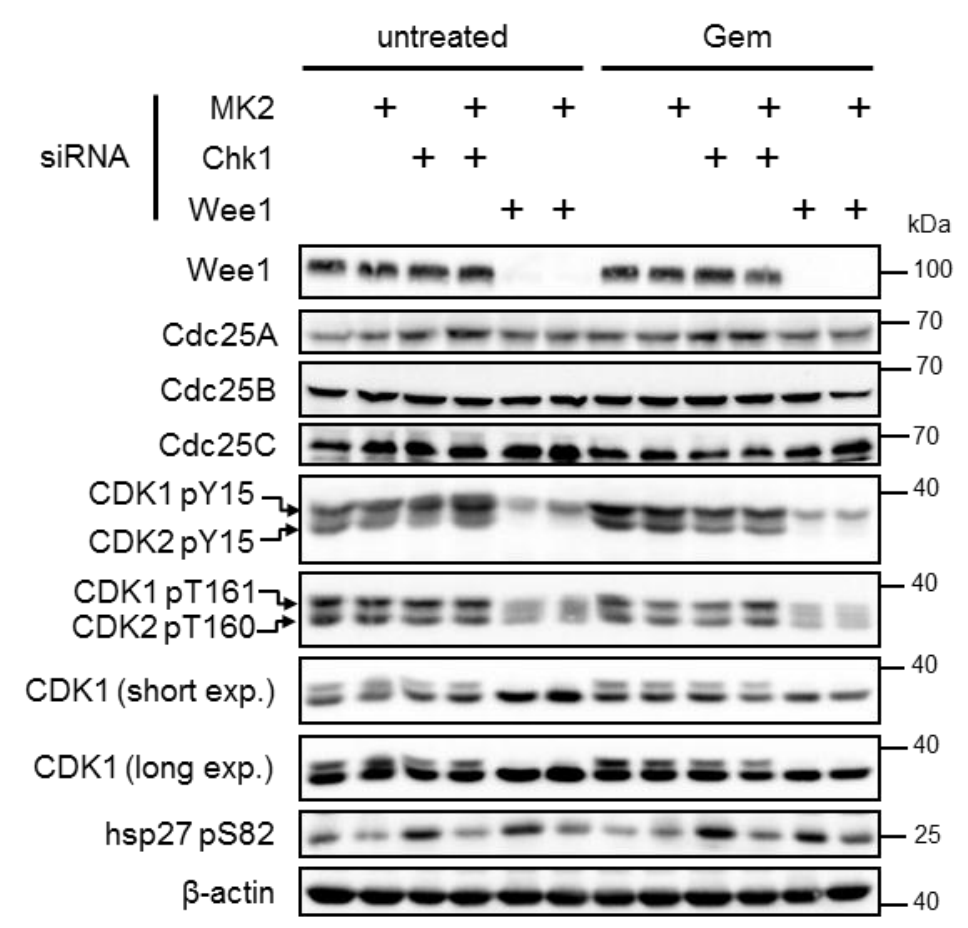

Figure V.19. Depletion of MK2 and Chk1 does not affect several regulators of origin firing. Cells were synchronized in G1 and depleted of MK2, Chk1 and Wee1 by siRNA-mediated knockdown. Then, cells were released into S-phase and after $2 \mathrm{~h}$ treated with $1 \mu \mathrm{M}$ gemcitabine for $2 \mathrm{~h}$ or left untreated. Cells were harvested and lysates analyzed by immunoblotting.

be a master regulator of origin firing, we hypothesized that origin firing is directly modulated by MK2 in this context and that the changes in replication fork speed merely represent a secondary effect.

To test this, we assessed protein levels of known regulators of origin firing, namely Cdc25 proteins and CDK1. We also investigated the phosphorylation status of CDK1 and CDK2.

Wee1 depletion efficiently impairs phosphorylation of CDK1 and CDK2 and served as a control. Cdc25s, CDK1 and CDK2 were all reported to be controlled by Chk1, and Cdc25A levels have been identified to depend on MK2. However, we found that neither 
Chk1 nor MK2 depletion affected Cdc25 or CDK1 protein levels. Moreover, CDK1/2 phosphorylation at the inhibitory $\mathrm{Y} 15$ also remained unchanged. Another point of regulation is the phosphorylation of CDK1 T161 and CDK2 T160. Phosphorylation of these residues was found to be required for complete activation of CDK1/2 (Gu et al., 1992; Norbury et al., 1991). CDK2 T160 was reported to be phosphorylated by Chk1 (Bourke et al., 2010), and the phosphorylation site is a close match for the Chk1/MK2 target motif. However, levels of CDK1 pT161 and CDK2 pT160 did not change upon knockdown of Chk1 and MK2 (Figure V.19).

In summary, we could not find any proof that Chk1 indeed regulates the levels of Cdc25 proteins or CDK1/2 during replication. MK2 also did not affect any of these proteins or their post-translational modification. Even Cdc25A levels, reported to be controlled by MK2 (Xiao et al., 2006) remained unchanged. Intriguingly, depletion of Wee1, used as a control for CDK1/2 pY15 in this experiment, had strong effects on the levels of CDK1 and also affected CDK1 pT161 and CDK2 pT160 (Figure V.19). While its influence on CDK1 activity via phosphorylation of $\mathrm{Y} 15$ has been investigated in detail, to our knowledge the finding that Wee1 depletion also impacts on CDK1 levels and T160/161 phosphorylation of CDK $1 / 2$ is a novelty.

\section{V.4 Localization and activation of MK2}

\section{V.4.1 Upon genotoxic stress MK2 is not completely exported from the nucleus}

The above results suggest a regulatory function of MK2 in DNA replication. All immediate regulation of replication takes place in the nucleus. It was therefore of interest to investigate whether some MK2 resides in the nucleus upon DNA damage, which can be regarded as a prerequisite for a role in immediate replication control.

In response to stress, induced by e.g. sorbitol or anisomycin, MK2 is rapidly phosphorylated and exported from the nucleus (Engel et al., 1998). It was shown that this is also true following treatment with doxorubicin and cisplatin (Reinhardt et al., 2010).These drugs have a mechanism of action that is distinct from that of UV light and gemcitabine: Doxorubicin intercalates in the DNA and induces DSBs by inhibiting topoisomerase II; cisplatin crosslinks bases of the DNA, interfering with replication (Wagner and Karnitz, 2009). We addressed the question whether MK2 is also exported from the nucleus upon irradiation with UV light or gemcitabine treatment. Strikingly, while sorbitol induced a complete nuclear export of MK2, UV exposure and gemcitabine treat- 


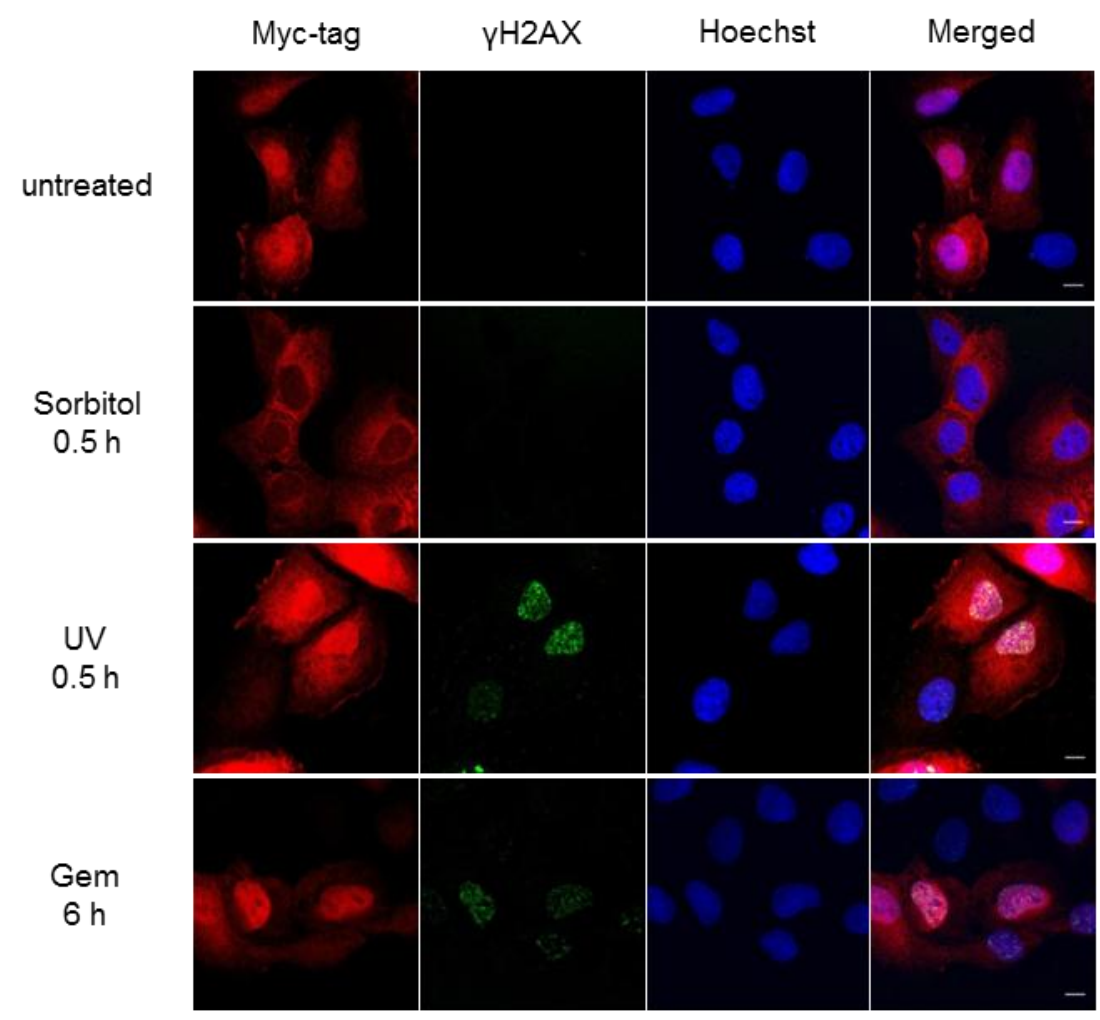

Figure V.20. Upon genotoxic stress, MK2 is not completely exported from the nucleus.

Myc-tagged MK2 was overexpressed in U2OS cells. Cells were treated with $0.4 \mathrm{M}$ sorbitol, 40 $\mathrm{J} / \mathrm{m}^{2}$ UV-C light or $100 \mathrm{nM}$ gemcitabine for the indicated time, then fixed and stained for confocal microscopy. Scale bar: $10 \mu \mathrm{m}$.

ment failed to do so (Figure V.20). Thus, it can be expected that MK2 activity following DNA damage induced by these agents is localized to the nucleus to a substantial degree. It is therefore possible that MK2 exhibits direct control on the replication machinery.

\section{V.4.2 p38 is required for Hsp27 phosphorylation but not for MK2-dependent DNA damage signaling}

It is still not completely understood how MK2 is activated in response to DNA damage. General stress signals activate MAP kinase signaling, which eventually leads to the phosphorylation and activation of p38 and p38 then activates MK2. For genotoxic stress, the upstream kinases required for MK2 phosphorylation appear to vary depending on the exact damaging agent (Reinhardt et al., 2007), indicating that one common pathway of activation is unlikely. When investigating the activity of MK2 in response to stress, previous reports exclusively focused on the phosphorylation of T334 as a readout for MK2 


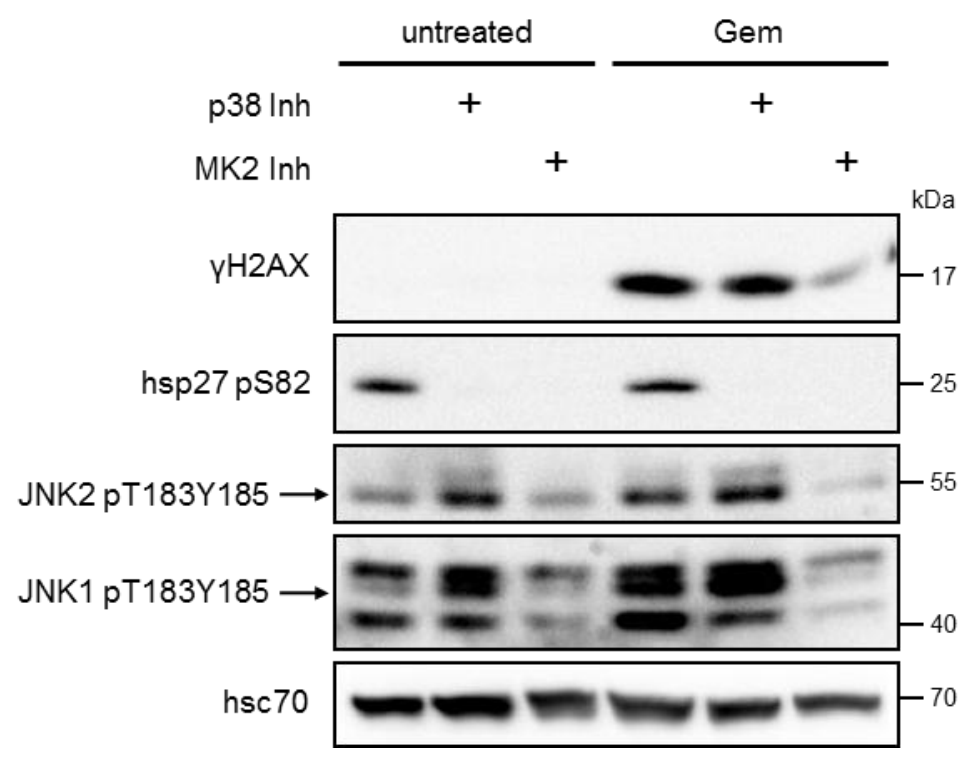

Figure V.21. Inhibition of MK2 but not of p38 impairs gemcitabine-induced H2AX and JNK phosphorylation.

Cells were treated with $200 \mathrm{nM}$ gemcitabine for $10 \mathrm{~h}$ in the presence of MK2 Inh, p38 Inh or DMSO. Thereafter, cells were harvested and cell lysates analyzed by immunoblotting.

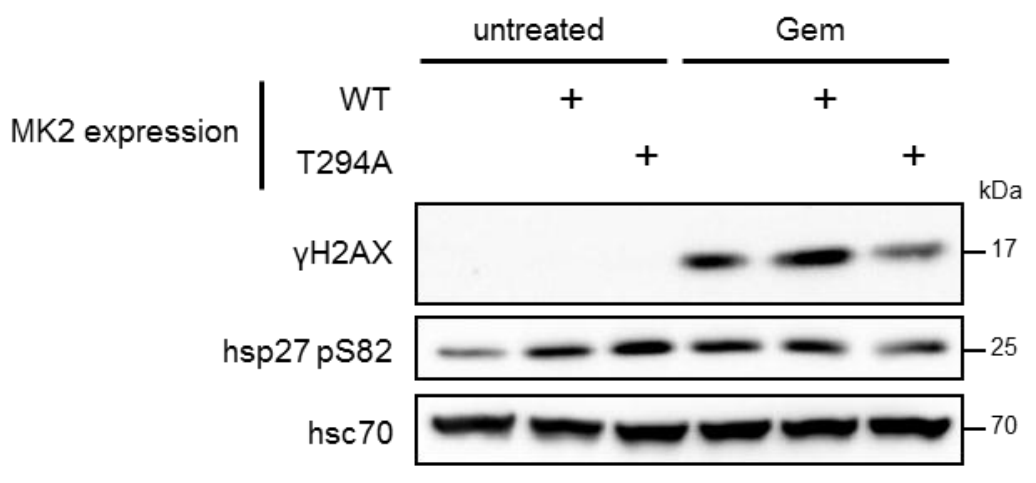

Figure V.22. MK2 mutated at a putative ATM/ATR phosphorylation site impairs gemcitabine-induced H2AX phosphorylation.

Cells were transfected with Myc-MK2 WT or a Myc-MK2 construct with the amino acid substitution T294A and treated with $100 \mathrm{nM}$ gemcitabine for $20 \mathrm{~h}$. Thereafter, cells were harvested and cell lysates analyzed by immunoblotting.

activity. However, this phosphorylation only marks the activity of p38. For this reason, we checked the levels of Hsp27 phosphorylation on Ser 82 as a measure for p38-induced MK2 activity, paying notion to the possibility that MK2 might be subject to additional regulation by other pathways.

We thus tested whether inhibition of MK2 and p38 have different outcomes regarding Hsp27 and H2AX phosphorylation upon gemcitabine-induced genotoxic stress. For 
inhibition of p38, we used the pharmacological inhibitor SB203580 ((Cuenda et al., 1995); called p38 Inh from here on). While MK2 inhibition, as observed before, impaired gemcitabine-induced Hsp27 and H2AX phosphorylation, p38 inhibition only affected Hsp27 phosphorylation; levels of $\mathrm{YH} 2 \mathrm{AX}$ were unchanged when compared to the DMSOtreated control (Figure V.21). Surprisingly, also JNK phosphorylation was only dependent on MK2 but not on p38 activity. This finding suggests that, in the DDR, MK2 is activated by a p38-independent pathway. Reduced phosphorylation of JNKs is probably attributed to an overall reduced DDR upon MK2 inhibition.

Likely candidates for a regulatory function with respect to MK2 are the very upstream DNA damage sensors ATM, ATR and DNA-PK. These kinases have a consensus phosphorylation motif comprised of the phosphorylated serine or threonine residue followed by glutamine; the motif is thus called $\mathrm{pS} / \mathrm{T}-\mathrm{Q}$. Hypothesizing that MK2 might be a direct substrate of one or more of these kinases, we searched for accessible $\mathrm{pS} / \mathrm{T}-\mathrm{Q}$ motifs on MK2. While so far no such phosphorylation-sites have been described for MK2, we found a potential phosphorylation site at T294.

Next, we wanted to test whether this site indeed serves a regulatory function for MK2. To this end, we constructed a non-phosphorylatable mutant form of MK2 carrying an amino acid substitution at this site, replacing Thr 294 with Ala (the mutant being called MK2 T294A). We over-expressed MK2 T294A in U2OS cells and analyzed the effect on Hsp27 and $\mathrm{H} 2 \mathrm{AX}$ phosphorylation following gemcitabine treatment (Figure V.22). Intriguingly, compared to MK2 WT, MK2 T294A impaired gemcitabine-induced H2AX phosphorylation, while phosphorylation of Hsp27 remained unchanged.

These results suggest that T294 might constitute a new regulatory phosphorylation site on MK2 that is specifically phosphorylated upon genotoxic stress and required for efficient accumulation of H2AX. Thus, p38 is only required for MK2-mediated phosphorylation of Hsp27 but not for DNA damage signaling downstream of MK2.

\section{V.5 MK2 in translesion synthesis}

\section{V.5.1 PCNA mono-ubiquitination induced by Chk1 depletion depends on MK2}

The effect of MK2 inhibition and knockdown on the genotoxic consequences of Chk1 impairment shows striking parallels to data that was published for the endonuclease Mus81. Mus81 has been reported to specifically cut ssDNA at stalled replication forks, thereby inducing DSBs, and Mus81 depletion can partially rescue reduced fork progression caused by Chk1 inhibition (Forment et al., 2011; Kai et al., 2005). In yeast, 


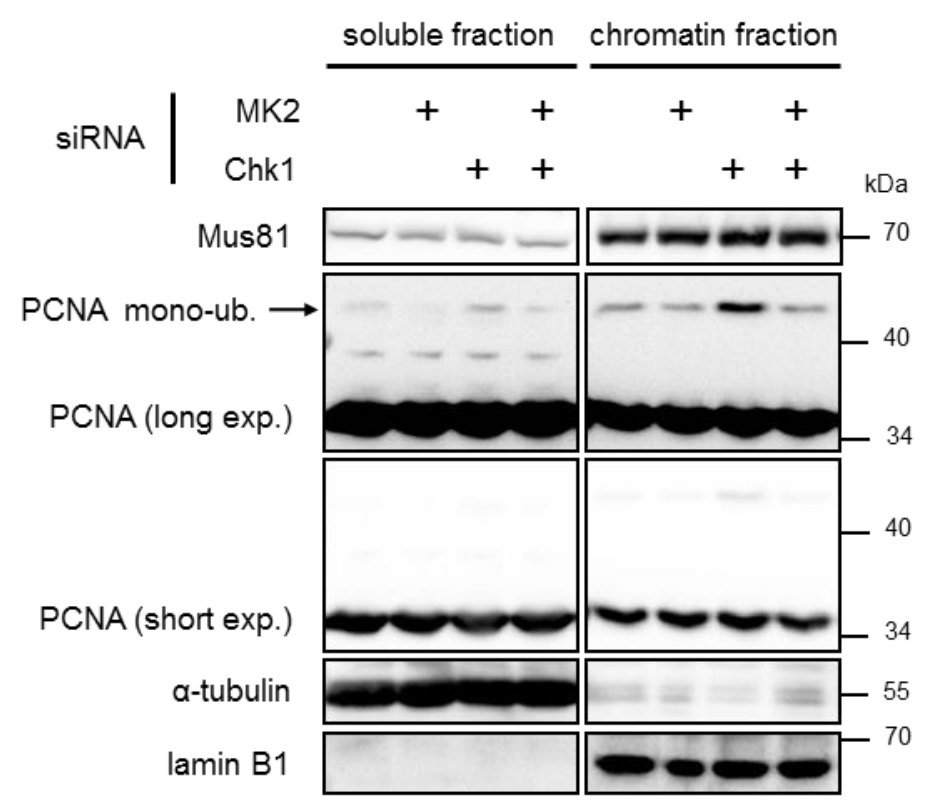

Figure V.23. PCNA mono-ubiquitination induced by Chk1 depletion depends on MK2.

Cells were synchronized in G1 and depleted of MK2 and Wee1 by siRNA-mediated knockdown, then released into S-phase. $5 \mathrm{~h}$ after release, cells were harvested and separated into soluble and chromatin fraction to analyze association of proteins with chromatin by immunoblotting. Monoubiquitination of PCNA induces a size shift of approx. $10 \mathrm{kDa}$.

Mus81 association to chromatin is regulated by the kinase Cds1 (Kai et al., 2005), but the kinase responsible for destabilization of Mus81 from chromatin in mammals remains to be identified. In the light of these findings, we wanted to test whether the effects we observed for MK2 depletion and inhibition are possibly mediated by Mus81 and whether Mus81 association with chromatin is subject to regulation by MK2. Furthermore, we assessed whether chromatin recruitment of PCNA as a central factor of replication was changed upon MK2 depletion.

Using chromatin fractionation of synchronized cells in S-phase, we found that depletion of neither Chk1 nor MK2 affects Mus81 chromatin association (Figure V.23). We also did not observe any changes in the recruitment of PCNA to chromatin. However, upon Chk1 depletion we observed an increase in a band corresponding to the size of monoubiquitinated PCNA in the chromatin fraction upon Chk1 depletion. PCNA monoubiquitination is associated with translesion synthesis in response to the occurrence of DNA lesions. While some publications claim Chk1 to be required for PCNA monoubiquitination (Bi et al., 2006; Yang et al., 2008) it has been argued that this effect most likely arises from decreased fork stability upon Chk1 depletion (Speroni et al., 2012). Interestingly, we further found that knockdown of MK2 reverted this effect, further arguing 
that the removal of MK2 attenuates the replicative stress imposed by Chk1 knockdown and possibly promotes fork stability.

\section{V.5.2 Translesion synthesis is required for the rescue of gemcitabine-induced replication impairment by MK2 inhibition}

As detailed above, our results show that DNA replication is impaired upon treatment with gemcitabine and that this impairment requires MK2 (Figure V.11). This finding raises the question of how MK2 mediates this block, and how replication is rescued upon inhibition of MK2, despite the continuous presence of gemcitabine.

One way to overcome replication blocks is TLS. Cells deficient in the translesion polymerase Pol $\eta$ display an increased sensitivity towards gemcitabine (Chen et al., 2006). Pol $\zeta$, a second TLS polymerase, is specialized to synthesize DNA from a distorted DNA duplex (Waters et al., 2009). An altered local structure of the DNA duplex has been described for gemcitabine and is the likely reason for replicative polymerase stalling (Konerding et al., 2002), suggesting a role for Pol $\zeta$ to overcome gemcitabine-induced replication blocks by TLS.

We hypothesized that TLS may not reach its full activity in the presence of MK2 but may efficiently overcome gemcitabine-induced lesions when MK2 is inactivated. In such a scenario, one would predict that MK2 inhibition can no longer rescue the gemcitabineinduced block in replication when TLS is impaired. To test this, we depleted cells of Pol $\eta$ and Rev3L, the catalytic subunit of Pol $\zeta$. Figure V.24a provides evidence of efficient knockdown of mRNA levels. We then assessed replication fork speed by DNA fiber assays upon gemcitabine treatment and MK2 inhibition as before (Figure V.24b). Figure V.24c shows the resulting average replication fork speeds. The removal of Pol $\eta$ and Rev3L did not grossly affect replication in the absence of gemcitabine, underscoring that in unperturbed cells, TLS is not essential. However, in the absence of these polymerases, MK2 inhibition was no longer capable of reversing the effects of gemcitabine on replication fork speed. The changes in fork speed are also documented by the distribution of fork rates in unperturbed and gemcitabine-treated cells (Figure V.24d). Figure V.24e shows corresponding representative images of the forks. Thus, upon the knockdown of these TLS components, gemcitabine slowed down the fork rate regardless of MK2.

We conclude that MK2 inhibition can only rescue the deleterious effects of gemcitabine on DNA replication when the TLS machinery is intact. These findings imply that MK2 acts as a mediator of the replicative stress response. It appears to block or at least to limit the ability of a cell to overcome replicative stress by TLS. In the absence of MK2 activity, the cells are considerably more tolerant to replicative stress, but this resistance requires TLS. 
a
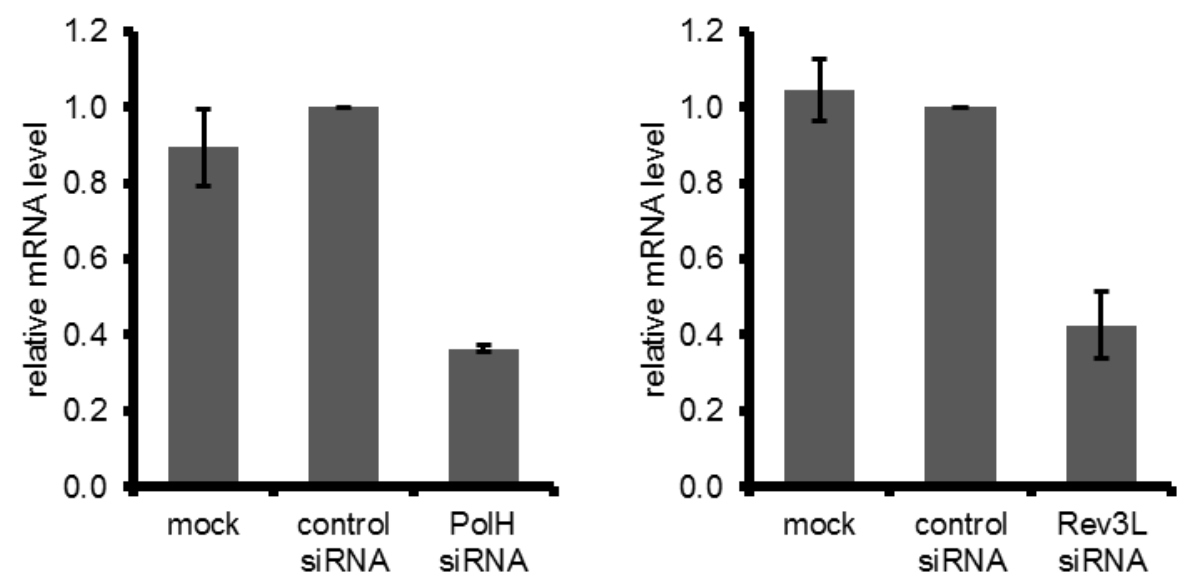

b

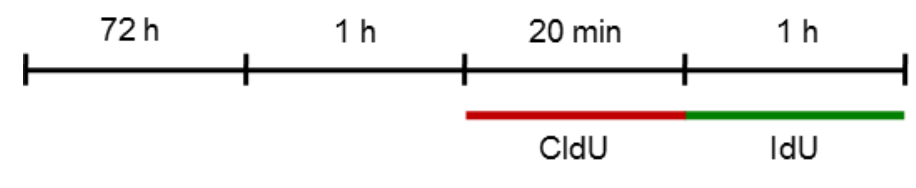

PolH + Rev3L knockdown

MK2 inhibition

Gem

C
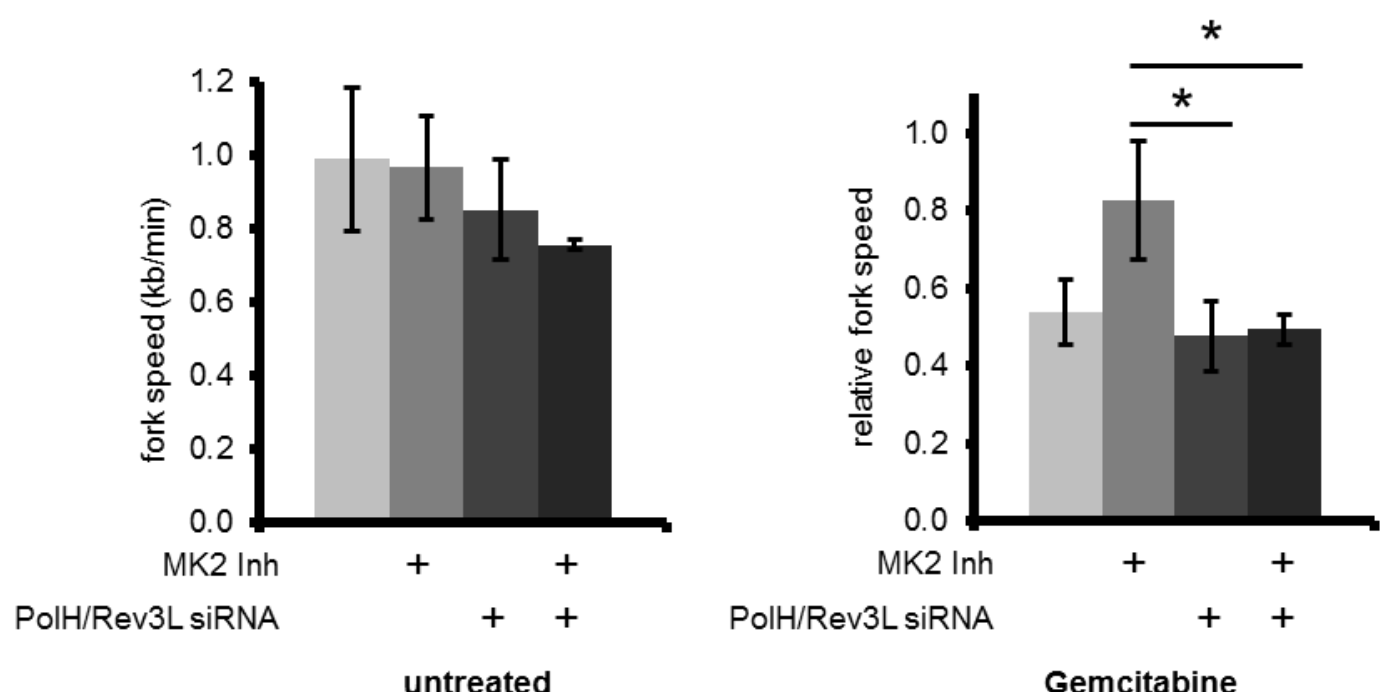

Figure V.24. Rescue of gemcitabine-induced slow replication fork speed by MK2 inhibition depends on translesion synthesis.

(a) Depletion efficiencies of PolH and Rev3L by siRNA-mediated knockdown. Cells were harvested $72 \mathrm{~h}$ after knockdown, mRNA was isolated and mRNA levels were analyzed by quantitative RTPCR. mRNA levels of PolH and Rev3L were normalized to GAPDH expression $(n=3)$. (b) Labeling protocol for DNA fiber analysis. U2OS cells were depleted of PolH and Rev3L by siRNAmediated knockdown. $72 \mathrm{~h}$ later, cells were pre-treated with MK2 Inh or DMSO for $1 \mathrm{~h}$ and then 
d

untreated

Gemcitabine
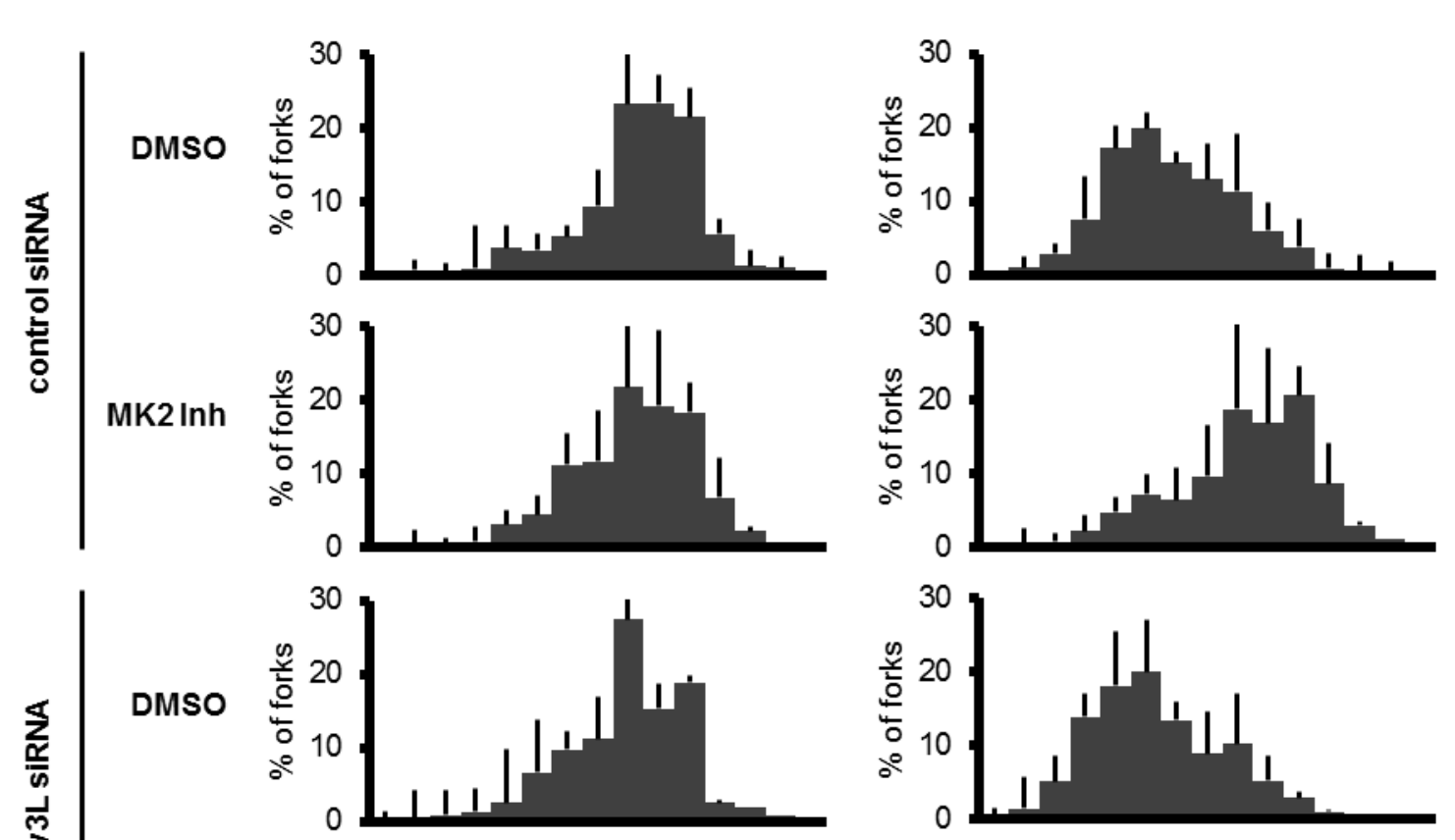

MK2 Inh
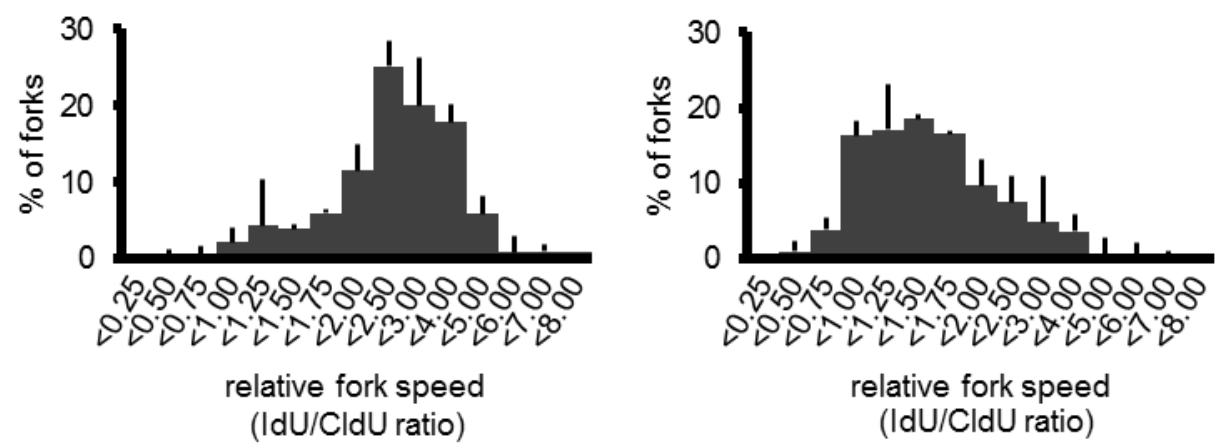

e

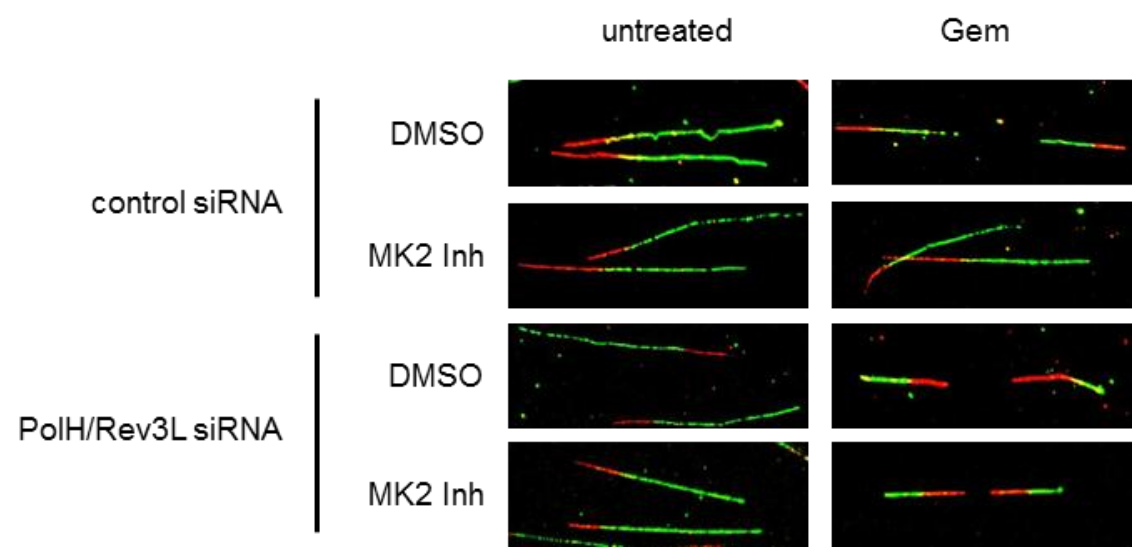

pulse labeled with CldU for 20 min. Afterwards, cells were pulse labeled with IdU for $1 \mathrm{~h}$ and simultaneously exposed to $400 \mathrm{nM}$ gemcitabine. CldU and IdU were detected using specific primary antibodies and secondary antibodies in red and green, respectively. (c) Absolute replication fork speed in untreated and relative replication fork speed (ratio of length of IdU-labeled tracks vs. length of CldU-labeled tracks) in gemcitabine-treated cells in dependence of MK2 inhibition and depletion of TLS polymerases PolH and Rev3L $(n=3)$. (d) Distribution of replication fork speeds in cells treated as in $\mathbf{b}$. (e) Representative images of fibers from cells treated as in $\mathbf{b}$. 


\section{DISCUSSION}

\section{VI.1 Tipping the balance: MK2 in the response to replicative stress}

The maintenance of genomic integrity is of vital importance to cells and multicellular organisms. Unrepaired damage to the DNA may result in cell death or uncontrolled proliferation, which can give rise to cancer. During the replication of the genome cells are exceptionally vulnerable to genotoxic stress. For this reason, DNA replication is tightly regulated and cells respond to replicative stress with a complex program to stabilize the genome, repair damage to the DNA and ensure that replication can be completed. The whole scope of the response to replicative stress only became known in recent years and more and more factors involved in the regulation of DNA replication are identified.

To shed more light on the cellular response to replicative stress, we investigated the contribution of the kinase MK2 to the DDR in S-phase. We found that MK2 is required for efficient H2AX phosphorylation following DNA damage caused by UV light and the nucleoside analog gemcitabine, suggesting that it participates in the very upstream part of DNA damage signaling. Importantly, this effect cannot be attributed to cell cycle arrest or DNA repair, which indicates that MK2 operates via a different mechanism in this context. Furthermore, MK2 inhibition or depletion protects cells from damage-induced cell death both in vitro and in vivo and promotes proliferation. We identified MK2 to regulate DNA replication in response to DNA damage. Reduced replication fork speed and increased origin firing caused by gemcitabine both depend on MK2. Moreover, MK2 is also required for the genotoxic effects of Chk1 inhibition and depletion, and MK2 inhibition rescues deregulated origin firing and replication fork progression in this context. MK2 is mostly retained in the nucleus following DNA damage caused by UV and gemcitabine, suggesting that it might exhibit direct regulation of the replication machinery. As we saw that MK2 inhibition only promotes fork progression when the TLS machinery is functional, we propose that MK2 is a direct modulator of TLS polymerase activity.

Whereas previous studies on MK2 in the DDR reported an involvement of the kinase in cell cycle checkpoint signaling, our data for the first time establishes a role of MK2 in the regulation of DNA replication. We thus demonstrate that the participation of MK2 in the DDR goes far beyond what has been known so far and identifies the kinase as a central signaling factor upon replicative stress. This new role of MK2 in replication must not surprise - in recent years, a number of proteins originally identified to be involved in G2/M 
checkpoint signaling were found to also control replication, among them kinases very central to the DDR such as CDK1 (Hochegger et al., 2007; Katsuno et al., 2009) and Wee1 (Beck et al., 2010; Dominguez-Kelly et al., 2011). Such a dual role may be explained by the necessity to coordinate these two processes to ensure that cells only enter mitosis after replication is completed.

In recent years it became increasingly clear that the persistent stalling of replication forks is not only a consequence of replicative stress but subject to regulation by DDR signaling, as well (Branzei and Foiani, 2010). While the details as to how prolonged replication fork stalling and stabilization are controlled are not fully understood, the significance of such regulation is undisputed. Extensive fork stalling implicates a substantial threat to genomic integrity since the global collapse of stalled forks into DSBs may result in genome fragmentation (Moldovan et al., 2007). Mechanisms that modulate fork progression or stability may therefore easily tip the balance between successful repair and cell survival on the one and cell death on the other hand. TLS is a very powerful tool to ensure fork progression upon DNA damage, favoring survival. However, there are two sides to every coin, and in the case of TLS survival is promoted at the expense of an increased mutation rate. Also, for multicellular organisms apoptosis of cells that suffered severe DNA damage can be favorable in certain situations to avoid uncontrolled proliferation and cancer. Hence, cells also evolved mechanisms to restrain TLS activity. The results presented in this work suggest that MK2 functions as part of the cellular machinery that represses TLS.

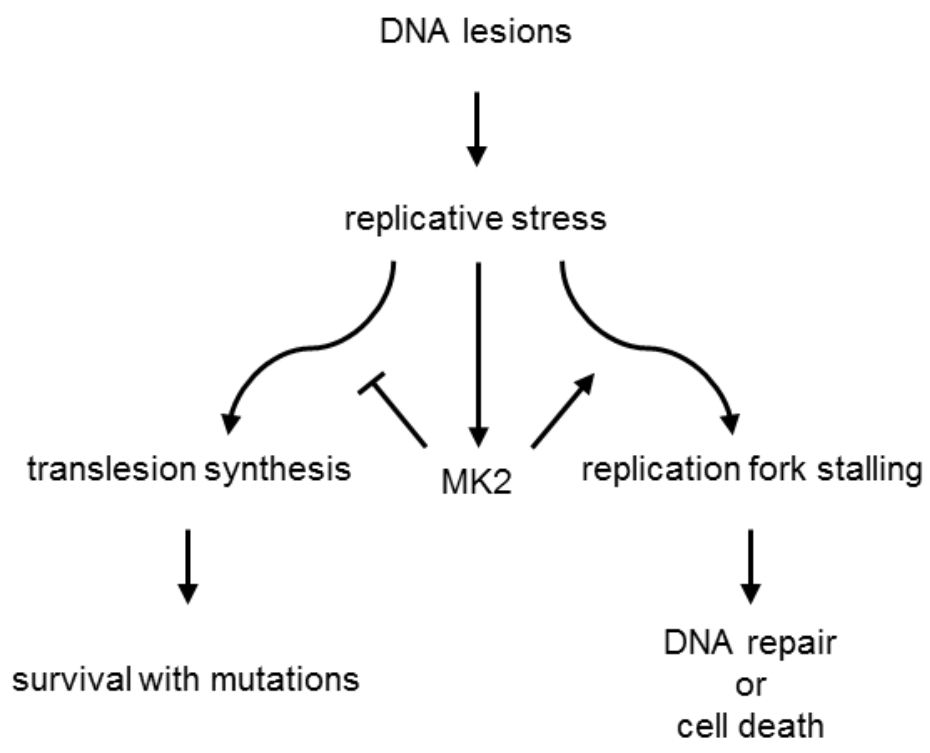

Figure VI.1. A model of how MK2 functions in replicative stress.

The decision between translesion synthesis on the one hand and replication fork stalling and DNA repair on the other upon replicative stress is regulated by MK2. See text for details. 
In such a scenario, MK2 exhibits control over the cellular decision between fork collapse and apoptosis on the one hand and fork stabilization and survival with an increased mutation rate on the other (Figure VI.1). Activation of MK2 by replicative stress limits TLS activity, promoting replication fork collapse and cell death. Impaired MK2 activity, on the contrary, supposedly increases TLS, resulting in fork stabilization and successful replication but increased mutagenesis.

In this context it is important to note that the power of TLS should be expected to lie in the promotion of fork progression upon low levels of DNA damage while severe genomic insults might require a different cellular response. In light of this, MK2 might function as a safeguard to restrain TLS when the DNA is severely damaged: Low levels of replicative stress would not fully activate MK2 and TLS activity would be maximal. Upon strong replicative stress, on the other hand, MK2 is strongly induced and represses TLS, promoting DNA repair or cell death.

According to this model, the decreased levels of $\mathrm{yH} 2 \mathrm{AX}$ observed upon MK2 inhibition or depletion reflect a reduced number of stalled replication forks and hence less DSBs. Enhanced cell survival and proliferation could thus also be attributed to increased TLS. In line with this model is our observation that MK2 inhibition or depletion in the absence of replicative stress does not impact DNA replication, $\mathrm{H} 2 \mathrm{AX}$ phosphorylation or cell viability: With no DNA damage present, negative regulation of TLS by MK2 is not required as TLS is inhibited by other cellular mechanisms.

\section{VI.2 Exploiting MK2 activity in chemotherapy}

Chemotherapy using DNA-damaging agents is a very effective way to treat various tumors, but for others the effects are limited and many cancer cells display resistance against chemotherapeutics. Great effort has therefore been made to identify factors that protect or sensitize cells to DNA damage and to develop drugs that specifically target these proteins (Medema and Macurek, 2012). This approach is based on the rationale that sensitization of cells by administration of such drugs in combination with chemotherapy results in synthetic lethality, i.e. the two chemicals kill the cell whereas each alone is not capable of doing so. A prominent example of such a drug target is Chk1, as its inhibition strongly sensitizes cells to DNA damage, and different Chk1 inhibitors are currently in clinical trials (Ma et al., 2011).

The work presented here identifies MK2 as a determinant of cellular sensitivity to UV irradiation and gemcitabine treatment. Interference with MK2 activity results in reduced DNA damage signaling and increased survival. These findings raise the question whether 
enhanced activation of MK2 might contribute to cell sensitization. This would be of great interest especially in the context of gemcitabine treatment of pancreatic carcinomas. Gemcitabine, although used in the first line treatment of this cancer type, only marginally extends survival, and resistance is an increasingly frequent problem (Long et al., 2011). The identification and manipulation of factors that determine gemcitabine sensitivity is therefore a promising approach to improve treatment efficacy (Hung et al., 2012).

The importance of the p38/MK2 pathway for the cellular response to nucleoside analogs has been reported before: It was recently found that p38 is critical for 5-FU-dependent apoptosis (de la Cruz-Morcillo et al., 2012). p38 is also required for cytotoxicity induced by gemcitabine in pancreatic cancer cell lines (Koizumi et al., 2005).

How could activity of p38 and MK2 be boosted? The p38/MK2 pathway is subject to negative regulation by the MAPK phosphatase 1 (MKP1 alias DUSP1). MKP1 dephosphorylates p38 T180 and Y182, thereby deactivating the kinase. Expression of MKP1 is enhanced by MK2 activity, establishing a negative feedback loop that restrains signaling via the p38/MK2 pathway (Hu et al., 2007).

Accordingly, interfering with MKP1 activity constitutes a promising possibility to promote the activity of p38 and MK2. In cultured cells, depletion of MKP1 results in enhanced activity of the pathway (personal communication with Veena Jagannathan). Furthermore, the natural diterpene triptolide interferes with MKP1 expression. It has been demonstrated that triptolide sensitizes cells to 5-FU (Chen et al., 2010) and we found the same to be true for gemcitabine (data not shown). However, triptolide affects many cellular processes and is highly toxic itself. It is therefore preferable to use more specific pharmacological inhibitors to interfere with MKP1 activity. Studies in our lab already proofed that such MKP1 inhibition sensitizes leukemic cells to DNA damage induced by the nucleoside analog cytarabine (personal communication with Veena Jagannathan). In spite of the inhibitor's specificity, however, side effects of MKP1 inhibition are still observed, which is probably due to the fact that MKP1 does not only target p38 but also other kinases such as JNKs and ERKs.

In summary, enhancing the activity of the p38/MK2 pathway is a promising approach to sensitize cells to cytotoxicity induced by nucleoside analogs. MKP1 constitutes a potential target to achieve this goal, but further research into this matter is required to thoroughly understand how manipulation of MKP1 and the p38/MK2 pathway interferes with other cellular processes. 


\section{VI.3 MK2 in checkpoint signaling and apoptosis}

We observe that knockdown or inhibition of MK2 reduces the H2AX phosphorylation induced by UV light or gemcitabine and protects cells from the consequences of DNA damage. In contrast, a number of studies using the same cell line report that MK2 is required for the $S$ and $G 2 / M$ checkpoint and that UV-induced DNA damage results in mitotic catastrophe in the absence of MK2 (Manke et al., 2005; Reinhardt et al., 2007; Reinhardt et al., 2010). We could not identify any effect of MK2 depletion on checkpoint functionality, but this might be due to differences in the experimental setup.

Manke and colleagues observe that knockdown of MK2 results in cell cycle progression through S-phase in spite of persistent DNA damage. They conclude that MK2 knockdown induces a loss of the S-phase checkpoint. This argumentation, however, is for two reasons not entirely convincing. Firstly, the S-phase checkpoint is pivotal also during unperturbed replication. Therefore, if MK2 depletion indeed abrogated the S-phase checkpoint, it should also result in replicative stress in the absence of exogenous DNA damage, as is the case for ATR and Chk1 depletion, two kinases that are indeed essential for S-phase arrest (Sorensen and Syljuasen, 2012). However, Manke and colleagues as well as our data (Figure V.10) clearly show that MK2 depletion does not affect unperturbed S-phase progression. Secondly, depletion of Chk1 illustrates what happens when a kinase critical for the S-phase checkpoint is missing: It does not result in progression through S-phase, as is suggested by Manke and colleagues for MK2, but rather entails extensive arrest of replication forks, subsequent fork collapse and consequently accumulation of cells in S-phase (Syljuasen et al., 2005) (Figure V.15). If MK2 was indeed essential for the S-phase checkpoint, its inhibition should have the same effect. This view is further supported by our observation that depletion of MK2 rescues the replication block induced by Chk1 depletion and promotes S-phase progression (Figure V.15). In fact, Manke and colleagues observe the same effect for UV. This finding argues that MK2 is not required for the S-phase checkpoint but is rather involved in fork stabilization and replication.

In any case, the data regarding the effect of MK2 on cell survival after DNA damage remains contradictory: Different publications observe increased cell death in the absence of MK2 (Johansen et al., 2009; Manke et al., 2005; Reinhardt et al., 2010) that was put down to checkpoint failure and mitotic catastrophe. In contrast, our results (Figure V.13; Figure V.16) and those of others (Xiao et al., 2006; Zenvirt et al., 2010) demonstrate that MK2 depletion rescues the cytotoxic effects of Chk1 depletion, reducing DNA damage and improving cell viability. In the same publications it is also reported that depletion of 
MK2 reverts the sensitization to cisplatin, doxorubicin and irinotecan induced by Chk1 depletion, and we observed the same effect for gemcitabine (data not shown). Furthermore, our data outreach the findings of Xiao and colleagues and Zenvirt and colleagues in that, as reported, we found that depletion or inhibition of MK2 protects cells from the consequences of DNA damage induced by UV irradiation or gemcitabine also in the presence of Chk1.

As detailed previously, this effect might be attributed to improved fork stability, TLS and therefore enhanced replication in the absence of MK2. A second mechanism by which MK2 depletion might impair apoptosis involves the MK2 substrate Hsp27: Following stress, MK2-mediated Hsp27 phosphorylation promotes the dissociation of Hsp27 oligomers, which is associated with apoptosis. MK2 knockout mice fail to phosphorylate Hsp25 (the homolog to human Hsp27) in response to stress and also display reduced apoptosis (Vertii et al., 2006). However, while these findings provide insight in how impairment of MK2 might promote cell viability, the reasons for the contradictory results with regard to MK2 and apoptosis remain elusive and necessitate further investigation, in particular since it is still unclear which pathways are responsible for the induction of apoptosis in response to replicative stress or checkpoint failure (Ewald et al., 2008).

\section{VI.4 Interplay between MK2 and Chk1}

It has been proposed that MK2 is a third checkpoint kinase, with a function analogous to Chk1 (Manke et al., 2005). The very similar substrate specificity clearly supports this view. In contrast, we found that genotoxic effects of Chk1 depletion or inhibition are rescued by depletion or inhibition of MK2. These results are in line with a study in HeLa cells that also found an antagonistic relationship between the two kinases (Xiao et al., 2006). This demonstrates that the genotoxic effects of Chk1 impairment depend on MK2 and suggests that a substantial subset of MK2 substrates is not shared by Chk1. How can the antagonistic action of the two kinases despite similar phosphorylation motifs be explained?

One possible mechanism for distinct functions of MK2 and Chk1 was brought up by the discovery that MK2 is exported into the cytoplasm upon certain kinds of genotoxic stress (treatment with cisplatin and doxorubicin) while Chk1 remains nuclear (Reinhardt et al., 2010). It is important to note, however, that neither nucleoside analogs nor UV irradiation were used as sources of DNA damage in that study. Both induce DNA damage signaling very different from that elicited by cisplatin and doxorubicin, and our results show that MK2 is retained in the nucleus following exposure to these agents (Figure V.20). 
This finding argues that different subcellular localization cannot account for the different effects we observe for MK2 and Chk1 in the DDR. Rather, the fact that we find a dominant negative effect of ectopically expressed MK2 carrying a mutation of a potential ATM/ATR phosphorylation site and that p38 inhibition does not reduce $H 2 A X$ phosphorylation following DNA damage suggests a more complex regulation of MK2 localization: Upon general stress, e.g. by sorbitol treatment, MK2 is solely activated by p38, resulting in nuclear export and phosphorylation of cytosolic Hsp27. Upon genotoxic stress caused by UV irradiation or gemcitabine, MK2 is additionally modified by a second pathway (possibly ATM/ATR acting on MK2 T294), which impairs complete nuclear export and thus increases the nuclear activity of MK2, entailing reduced phosphorylation of H2AX.

Another reason for functional divergence between the two kinases might be that their substrate phosphorylation motifs are similar but not identical. While both kinases require a large hydrophobic residue in the -5 position of their substrate and arginine in the -3 position, the other positions, although less important for phosphorylation, display differences: MK2 favors glutamine in the -2 and leucine or asparagine in the -1 site whereas Chk1 prefers tyrosine and glutamate, respectively (Manke et al., 2005). This divergence, however, may account for minor differences in the substrate spectrum but is unlikely to result in completely different functionality.

Finally, if both kinases are localized to the nucleus, share a similar substrate specificity and are activated independently from each other upon DNA damage, it seems most convincing to us that their antagonistic action results from a different spectrum of interaction partners. Apart from their substrate specificity MK2 and Chk1 are structurally unrelated and have different domains that mediate protein-protein interactions. MK2, for instance, can interact with proteins harboring an SH3-domain via its proline-rich $\mathrm{N}$ terminus. However, significant interactions with SH3-domains have not been found for MK2 so far. Interaction with other proteins could also be mediated by $\mathrm{p38}$, which forms a stable complex with MK2. Chk1, on the other hand, contains a PIP-box that facilitates interaction with PCNA. In fact, it has been demonstrated that this PIP-box is required for the DNA damage-induced release of Chk1 from chromatin and also to promote replication fork progression (Speroni et al., 2012). The interaction partners are thus likely responsible to direct MK2 and Chk1 to their specific substrates by either mediating direct interaction between kinase and substrate or by controlling the kinases' sub-nuclear localization, increasing their local concentration and bringing them in close proximity to potential substrates. 
The divergence between MK2 and Chk1 is well exemplified by their separate roles in the regulation of Hsp27 and origin firing.

Hsp27 S82 is exclusively phosphorylated by MKs in response to stress. It is not only localized in the cytoplasm but was also found in sub-nuclear structures (Vos et al., 2009) and could thus be expected to be a substrate of Chk1, as well. However, a dependence of Hsp27 phosphorylation on Chk1 has not been observed so far, and in our hands Chk1 inhibition or depletion does not decrease Hsp27 phosphorylation (Figure V.13). On the contrary, we observe an increase of Hsp27 pS82 upon Chk1 inhibition, which is likely attributed to increased replicative stress. This finding illustrates that, in the case of MK2 and Chk1, identical in vivo substrates cannot be predicted from a shared phosphorylation motif.

The same holds true for their role in origin firing. Chk1 is a master regulator of origin firing whereas MK2 does not seem to play a direct role in this process. This is also supported by our observation that the effect of Wee1 depletion, which also leads to deregulated origin firing (Sorensen and Syljuasen, 2012), does not depend on MK2 (Figure V.14). As detailed before, the increased origin firing observed upon Chk1 depletion or inhibition is caused by activation of previously inactive origin clusters as well as stochastic firing of dormant origins due to replication fork stalling (Blow and Ge, 2009; Ge and Blow, 2010). Opposed to Wee1, Chk1 has been directly implicated in fork stabilization. Since we could not identify a role for MK2 in the regulation of various factors involved in origin firing (Figure V.19), the rescue of origin firing upon MK2 inhibition is probably indirect and attributed to MK2 activity directed towards fork destabilization by repression of TLS. Thus, origin firing could serve as an example for very distinct functions of MK2 and Chk1, the former destabilizing forks via TLS repression, the latter regulating origin firing and stabilizing forks. The target of MK2 via which it exhibits its control on TLS, however, remains to be identified.

Further support for substantial functional differences between Chk1 and MK2 in spite of similar substrate specificity comes from mice in which loss of Chk1 confers embryonic lethality while MK2 ablation does not affect viability (Kotlyarov et al., 1999; Liu et al., 2000).

It remains to be seen which factors target MK2 and Chk1 to different substrates. For Chk1, co-factors like Claspin are already known and its substrate spectrum has been analyzed in detail. MK2, on the other hand, appears to be implicated in more cellular processes than known so far, and thus new putative substrates emerge. 


\section{VI.5 MK2 in the regulation of translesion synthesis}

In this work we identify the protective effects of MK2 inhibition to depend on TLS following gemcitabine-induced replicative stress. So far we have not analyzed whether this is also true for replicative stress induced by UV irradiation. However, a similar dependence of the effect of MK2 inhibition on TLS in the context of UV-induced DNA damage is a plausible scenario as Pol $\eta$ is essential for TLS upon both kinds of lesions. This is further supported by the fact that we found impairment of MK2 activity to have the same effect on H2AX phosphorylation and cell viability following both gemcitabine treatment and exposure to UV irradiation.

As detailed above, restraining TLS activity is pivotal to cells as this damage tolerance mechanism goes along with increased mutagenesis and must be restrained unless its activity is required. DNA damage tolerance is considered as a means for cells to ensure S-phase progression despite genotoxic stress. Interestingly, it has been speculated that the most important function of the S-phase checkpoint is to allow time for DNA damage tolerance mechanisms such as TLS and re-priming rather than DNA repair (Koren, 2007). Accordingly, our observation that MK2 depletion alleviates cells of the S-phase arrest induced by Chk1 depletion (Figure V.15) also argues that MK2 affects TLS and not DNA repair. Consequently, this raises the question how MK2 acts on TLS.

In the work presented here, we depleted cells of Pol $\eta$ and the catalytical subunit of Pol $\zeta$ to deactivate TLS. The fact that this interferes with the effect of MK2 inhibition, however, does not mean that the activity of MK2 is necessarily immediately directed against one of these polymerases. Regulation of TLS takes place on different levels that are discussed below: MK2 might target the mono-ubiquitination of PCNA via Rad6 and Rad18, the loading and phosphorylation of the 9-1-1 complex upon DNA damage, TLS polymerase expression, the polymerase switch at the lesion or polymerase activity.

We found that PCNAub1 is increased upon Chk1 depletion (Figure V.23), likely due to increased fork collapse (Jansen et al., 2007). This increase is rescued by co-depletion of MK2. One might speculate that this rescue is due to a direct role of MK2 in PCNA monoubiquitination. However, such a function of MK2 would induce TLS rather than repress it. Also, no connection between MK2 and ubiquitination has been established so far. The two ubiquitin ligases responsible for ubiquitination of PCNA at K164, Rad6 and Rad18, are phosphorylated on different sites, possibly by CDK1 and CDK2 (Chi et al., 2008; Sarcevic et al., 2002) as well as ATM and ATR (Wang et al., 2005). However, how these phosphorylations influence the enzymes' ubiquitin ligase activity is not clear. None of the known phosphorylation sites on Rad6 and Rad18 match the MK2 target motif, making it unlikely that MK2 acts at this level. Rather, the decrease in PCNAub1 upon MK2 
depletion might reflect improved fork stability, possibly due to an increase in TLS. This is not necessarily a contradiction since TLS promotes fork stability, reducing ssDNA and thereby the stimulus for PCNAub1.

Loading of the 9-1-1 complex that also participates in the recruitment of TLS factors is stimulated by ssDNA. It is loaded by the RFC-like clamp loader Rad17-RFC. In Rad17RFC, the co-factor p140, which provides specificity for PCNA, is replaced by Rad17. Rad17 is extensively phosphorylated, also by ATR (Medhurst et al., 2008), but the significance of these phosphorylations is not completely understood. The Rad17 phosphorylation site T304, however, is located in the motif $L-N-R-I-V-p T-I$ and thus a good but not perfect match for MK2. If MK2 exhibits regulation on this level and represses 9-1-1 loading, one consequence should be reduced recruitment of TLS polymerases to stalled replication forks. Thus, depletion or inhibition of MK2 would be expected to promote re-localization of TLS polymerases upon replicative stress.

The significance of 9-1-1 phosphorylation has been investigated in more detail. Phosphorylation of 9-1-1 is required for the recruitment of TLS polymerases and also contributes to ATR activation via TopBP1 (Delacroix et al., 2007). Yet, none of the three 9-1-1 components Rad9, Hus1 and Rad1 contains phosphorylation sites that fit MK2 and therefore a regulation of TLS recruitment via 9-1-1 by MK2 is not likely.

Following polymerase recruitment, the switch from the replicative polymerase to a TLS polymerase at the site of a lesion is most probably also tightly controlled. Rad6/Rad18dependent ubiquitination plays a role here, but as with many other facets of TLS details remain elusive. It is therefore not possible to judge at this point whether MK2 participates in this process.

The following levels of TLS control target the polymerases themselves. Expression of TLS polymerases is subject to tight regulation. This especially applies to Pol $\zeta$ and Rev1 (Waters et al., 2009). Since MK2 has been implicated in the regulation of protein expression via mRNA stabilization, it is tempting to speculate that MK2 also exhibits control of TLS polymerase expression. However, the effects observed in the fiber assay upon MK2 inhibition are too rapid to be attributed to changes in protein levels. Importantly, this does not generally rule out that MK2 indeed targets TLS polymerase mRNA stability. Along the same line it is noteworthy that p53 was found to repress TLS polymerase expression, as well (Lin and Howell, 2006), but since we could not find differences in the overall effects of MK2 on the DDR between p53 WT (U2OS) and p53 mutant cell lines (e.g. BxPC-3, MIA PaCa-2), MK2 is unlikely to act via this pathway. Still, changes in TLS polymerase protein levels in dependence of MK2 and p53 should be subject to future investigation. 
TLS polymerases are huge proteins and subject to extensive posttranslational modification. Ubiquitination as a common theme of TLS appears to play a role in the direct regulation of TLS polymerase activity. Pol $\eta$, Pol I, Rev7 and Rev1 are all ubiquitinated. However, in almost all cases neither the relevance nor the responsible ubiquitin ligase is known. This also applies largely to polymerase phosphorylation. Many phosphorylation sites have been identified by mass spectrometry but await further investigation, and it is undisputed that control of TLS is exhibited by pathways independent of PCNA and the 9-1-1 complex (Branzei and Foiani, 2007). Thus, a direct modulation of TLS by MK2-mediated phosphorylation of the responsible polymerases is a likely scenario. Phosphorylation might either affect polymerase activity or interaction with other proteins.

One candidate substrate would thus be Rev1 as a central factor of the TLS machinery, required for the recruitment and arrangement of TLS components. It interacts with other TLS polymerases as well as with PCNA and is pivotal to fork progression under replicative stress (Edmunds et al., 2008). A phosphorylation site that would match the MK2 target motif, however, has not been described so far.

Pol $\zeta$ is of comparable importance for TLS functionality as Rev1, illustrated by the fact that loss of Rev3, the catalytical subunit of Pol $\zeta$, confers embryonic lethality (Esposito et al., 2000). Regulation of its activity is thought to be mostly exhibited by the non-catalytic subunit Rev7. While Rev7 does not contain any phosphorylation fitting the MK2 target motif, the catalytical subunit Rev3 does. S1075 of Rev3 is located in the sequence I - K $\mathrm{R}-\mathrm{T}-\mathrm{L}-\mathrm{pS}-\mathrm{F}$ and a near-perfect match for MK2. To date, Rev3 has not been crystallized completely. It is therefore unknown whether this phosphorylation site is localized in a region required for protein-protein interaction or catalytic activity. Still, the existence of this phosphorylation site identifies Rev3 as a potential target for MK2.

Finally, Pol $\eta$, the TLS polymerase that has been directly associated with gemcitabine (Chen et al., 2006), also harbors a potential MK2 phosphorylation site, which is S380 in the sequence context $D-K-R-L-S-p S-L$. Interestingly, S380 is located in a protein domain that directly interacts with the upstream part of the bound DNA (Biertumpfel et al., 2010). Thus, phosphorylation of S380 might influence association of the polymerase with DNA, modulating its activity.

In summary, from what is known about the regulation of TLS to date, MK2 might control TLS at different levels and it is difficult to judge which one is most likely. Rev17, a factor of the 9-1-1 loading clamp, is a potential substrate. Regulation of 9-1-1 loading would interfere with TLS recruitment to the lesion. Alternatively, Pol $\zeta$ and Pol $\eta$ might be targeted by MK2. This would either affect the interaction with other proteins or directly modulate polymerase activity. 


\section{VI.6 Conclusions and future perspectives}

The findings presented here establish MK2 as a central player in the DDR. It regulates replication in a manner that is dependent on TLS. In fact, the repression of TLS by MK2 constitutes the first example of negative regulation of TLS as a result of DNA damage signaling. All previously reported damage-mediated control of TLS results in enhanced TLS activity (Jansen et al., 2007; Waters et al., 2009). It should not surprise that also negative regulation of TLS could be stimulated by DNA damage signaling. As pointed out before, TLS is highly mutagenic. Even in the presence of DNA damage, it is not always desirable for the cell to employ TLS, accepting the burden of an increased mutation rate, but rather attempt to cope with the damage via a different, less error-prone mechanism. Our findings suggest that other components of the DDR might contribute to the repression of TLS, as well. In general, the fine-tuning of TLS is only partly understood and demands further investigation.

In this context, the next step is to investigate whether MK2 interferes with TLS polymerase localization to stalled forks or whether it acts downstream of this recruitment process. Moreover, the identification of the substrate(s) of MK2 in the TLS machinery will certainly be helpful and would deepen our understanding of its function in this context. The fact that MK2 inhibition appears to promote TLS furthermore raises the question whether this goes along with increased mutagenesis. Conversely, it should be expected that enhanced MK2 activation reduces the TLS-associated mutation rate.

A thorough understanding of how MK2 impacts TLS might also be of value to unravel the contradiction between our data and publications that report a protective function of MK2 activity. To this end, it is also of interest to analyze the complete phosphorylation pattern of MK2 and to determine whether kinases other than p38 or ERKs are involved in MK2 phosphorylation and whether this influences the subcellular localization of MK2, as outlined above.

Furthermore, the connection between MK2 activity and apoptosis necessitates further study. Reports concerning the impact of MK2 on Hsp27-mediated apoptosis are partly contradictory and it is not clear whether a direct link exists. Also, potential cross-signaling between the p38/MK2 pathway and JNKs has not been studied in detail and might also constitute a way by which MK2 affects cell viability. Our findings suggest, however, that increased fork collapse in the presence of MK2 might trigger apoptosis so that MK2 only indirectly contributes to the induction of cell death. 
Finally, as discussed before, we can also only speculate about the mechanism underlying the antagonistic activity between MK2 and Chk1. The observed requirement of TLS to rescue gemcitabine-induced fork stalling can probably not be directly transferred to replicative stress caused by Chk1 inhibition. However, it was recently suggested that HR might depend on TLS to repair DSBs (Branzei and Foiani, 2007), giving rise to speculations that MK2 inhibition contributes to the repair of collapsed forks upon Chk1 inhibition by promoting TLS-dependent HR. Alternatively, MK2 might act on replication via different mechanisms. One such mechanism that promotes fork progression but has been poorly investigated so far is re-priming. Stalled forks can be rescued by re-priming, leaving a gap in the synthesized strand that can be filled post-replicatively (Branzei and Foiani, 2007). Importantly, these gaps are too small to be observable using fiber assays. Thus, increased track length upon treatment might in fact represent a re-priming event (Elvers et al., 2011). A separate assay would be required to distinguish between continuous and gapped tracks. However, it is intriguing to consider the possibility that MK2 might compromise fork stability by repressing both TLS and re-priming.

Summarized, the data presented here demonstrates that MK2 impairs fork progression upon replicative stress and this activity depends on TLS. MK2 confers sensitivity towards DNA damage, identifying the p38/MK2 pathway as a promising drug target to sensitize cells in chemotherapy. Potential MK2 substrates in the TLS machinery are the TLS polymerases Pol $\eta$ and Pol $\zeta$ as well as Rad17. Further studies are required to determine the details of the control exhibited by MK2. Also, persistent contradictions between different reports on the effect of MK2 in the DDR necessitate elucidation, one possibly crucial point of regulation being differential MK2 activation and localization.

It is becoming increasingly clear that cells actively control the decision between attempted DNA repair with the risk of damaged-induced cell death and tolerance pathways that go along with the burden of increased mutagenesis. This report for the first time describes a function of MK2 in DNA replication, which substantially extends the kinase's operating range in the DDR. MK2 appears to be one of the factors capable of tipping the balance, ruling over life and death. 


\section{REFERENCES}

Anderson, D.R., Meyers, M.J., Vernier, W.F., Mahoney, M.W., Kurumbail, R.G., Caspers, N., Poda, G.I., Schindler, J.F., Reitz, D.B., and Mourey, R.J. (2007). Pyrrolopyridine inhibitors of mitogen-activated protein kinase-activated protein kinase 2 (MK-2). Journal of medicinal chemistry 50, 2647-2654.

Bartek, J., Lukas, C., and Lukas, J. (2004). Checking on DNA damage in S phase. Nature reviews Molecular cell biology 5, 792-804.

Bartek, J., and Lukas, J. (2003). Chk1 and Chk2 kinases in checkpoint control and cancer. Cancer cell 3, 421-429.

Bartkova, J., Rezaei, N., Liontos, M., Karakaidos, P., Kletsas, D., Issaeva, N., Vassiliou, L.V., Kolettas, E., Niforou, K., Zoumpourlis, V.C., et al. (2006). Oncogene-induced senescence is part of the tumorigenesis barrier imposed by DNA damage checkpoints. Nature 444, 633-637.

Beck, H., Nahse, V., Larsen, M.S., Groth, P., Clancy, T., Lees, M., Jorgensen, M., Helleday, T., Syljuasen, R.G., and Sorensen, C.S. (2010). Regulators of cyclin-dependent kinases are crucial for maintaining genome integrity in $S$ phase. The Journal of cell biology 188, 629-638.

Ben-Levy, R., Hooper, S., Wilson, R., Paterson, H.F., and Marshall, C.J. (1998). Nuclear export of the stress-activated protein kinase p38 mediated by its substrate MAPKAP kinase-2. Current biology : CB 8, 1049-1057.

Ben-Levy, R., Leighton, I.A., Doza, Y.N., Attwood, P., Morrice, N., Marshall, C.J., and Cohen, P. (1995). Identification of novel phosphorylation sites required for activation of MAPKAP kinase-2. The EMBO journal 14, 5920-5930.

Bi, X., Barkley, L.R., Slater, D.M., Tateishi, S., Yamaizumi, M., Ohmori, H., and Vaziri, C. (2006). Rad18 regulates DNA polymerase kappa and is required for recovery from Sphase checkpoint-mediated arrest. Molecular and cellular biology 26, 3527-3540.

Bienko, M., Green, C.M., Crosetto, N., Rudolf, F., Zapart, G., Coull, B., Kannouche, P., Wider, G., Peter, M., Lehmann, A.R., et al. (2005). Ubiquitin-binding domains in Y-family polymerases regulate translesion synthesis. Science 310, 1821-1824.

Biertumpfel, C., Zhao, Y., Kondo, Y., Ramon-Maiques, S., Gregory, M., Lee, J.Y., Masutani, C., Lehmann, A.R., Hanaoka, F., and Yang, W. (2010). Structure and mechanism of human DNA polymerase eta. Nature 465, 1044-1048.

Blow, J.J., and Ge, X.Q. (2009). A model for DNA replication showing how dormant origins safeguard against replication fork failure. EMBO reports 10, 406-412.

Blow, J.J., Ge, X.Q., and Jackson, D.A. (2011). How dormant origins promote complete genome replication. Trends in biochemical sciences 36, 405-414.

Bourke, E., Brown, J.A., Takeda, S., Hochegger, H., and Morrison, C.G. (2010). DNA damage induces Chk1-dependent threonine-160 phosphorylation and activation of Cdk2. Oncogene 29, 616-624. 
Branzei, D., and Foiani, M. (2007). Interplay of replication checkpoints and repair proteins at stalled replication forks. DNA repair 6, 994-1003.

Branzei, D., and Foiani, M. (2010). Maintaining genome stability at the replication fork. Nature reviews Molecular cell biology 11, 208-219.

Brown, E.J., and Baltimore, D. (2003). Essential and dispensable roles of ATR in cell cycle arrest and genome maintenance. Genes \& development 17, 615-628.

Bulavin, D.V., Higashimoto, Y., Popoff, I.J., Gaarde, W.A., Basrur, V., Potapova, O., Appella, E., and Fornace, A.J., Jr. (2001). Initiation of a G2/M checkpoint after ultraviolet radiation requires p38 kinase. Nature 411, 102-107.

Burma, S., Chen, B.P., Murphy, M., Kurimasa, A., and Chen, D.J. (2001). ATM phosphorylates histone H2AX in response to DNA double-strand breaks. The Journal of biological chemistry 276, 42462-42467.

Chen, Y.W., Cleaver, J.E., Hanaoka, F., Chang, C.F., and Chou, K.M. (2006). A novel role of DNA polymerase eta in modulating cellular sensitivity to chemotherapeutic agents. Molecular cancer research : MCR 4, 257-265.

Chen, Y.W., Lin, G.J., Chuang, Y.P., Chia, W.T., Hueng, D.Y., Lin, C.K., Nieh, S., and Sytwu, H.K. (2010). Triptolide circumvents drug-resistant effect and enhances 5fluorouracil antitumor effect on KB cells. Anti-cancer drugs 21, 502-513.

Chi, Y., Welcker, M., Hizli, A.A., Posakony, J.J., Aebersold, R., and Clurman, B.E. (2008). Identification of CDK2 substrates in human cell lysates. Genome biology 9, R149.

Ciccia, A., and Elledge, S.J. (2010). The DNA damage response: making it safe to play with knives. Molecular cell 40, 179-204.

Cimprich, K.A., and Cortez, D. (2008). ATR: an essential regulator of genome integrity. Nature reviews Molecular cell biology 9, 616-627.

Clifton, A.D., Young, P.R., and Cohen, P. (1996). A comparison of the substrate specificity of MAPKAP kinase-2 and MAPKAP kinase-3 and their activation by cytokines and cellular stress. FEBS letters 392, 209-214.

Cortez, D., Guntuku, S., Qin, J., and Elledge, S.J. (2001). ATR and ATRIP: partners in checkpoint signaling. Science 294, 1713-1716.

Coxon, P.Y., Rane, M.J., Uriarte, S., Powell, D.W., Singh, S., Butt, W., Chen, Q., and McLeish, K.R. (2003). MAPK-activated protein kinase-2 participates in p38 MAPKdependent and ERK-dependent functions in human neutrophils. Cellular signalling 15, 993-1001.

Crul, M., van Waardenburg, R.C., Bocxe, S., van Eijndhoven, M.A., Pluim, D., Beijnen, J.H., and Schellens, J.H. (2003). DNA repair mechanisms involved in gemcitabine cytotoxicity and in the interaction between gemcitabine and cisplatin. Biochemical pharmacology 65, 275-282.

Cuenda, A., Rouse, J., Doza, Y.N., Meier, R., Cohen, P., Gallagher, T.F., Young, P.R., and Lee, J.C. (1995). SB 203580 is a specific inhibitor of a MAP kinase homologue which is stimulated by cellular stresses and interleukin-1. FEBS letters 364, 229-233. 
d'Adda di Fagagna, F. (2008). Living on a break: cellular senescence as a DNA-damage response. Nature reviews Cancer 8, 512-522.

Davis, R.J. (2000). Signal transduction by the JNK group of MAP kinases. Cell 103, 239252.

De Bont, R., and van Larebeke, N. (2004). Endogenous DNA damage in humans: a review of quantitative data. Mutagenesis 19, 169-185.

de la Cruz-Morcillo, M.A., Valero, M.L., Callejas-Valera, J.L., Arias-Gonzalez, L., MelgarRojas, P., Galan-Moya, E.M., Garcia-Gil, E., Garcia-Cano, J., and Sanchez-Prieto, R. (2012). P38MAPK is a major determinant of the balance between apoptosis and autophagy triggered by 5-fluorouracil: implication in resistance. Oncogene 31, 1073-1085.

Delacroix, S., Wagner, J.M., Kobayashi, M., Yamamoto, K., and Karnitz, L.M. (2007). The Rad9-Hus1-Rad1 (9-1-1) clamp activates checkpoint signaling via TopBP1. Genes \& development 21, 1472-1477.

Dominguez-Kelly, R., Martin, Y., Koundrioukoff, S., Tanenbaum, M.E., Smits, V.A., Medema, R.H., Debatisse, M., and Freire, R. (2011). Wee1 controls genomic stability during replication by regulating the Mus81-Eme1 endonuclease. The Journal of cell biology 194, 567-579.

Durkin, S.G., Arlt, M.F., Howlett, N.G., and Glover, T.W. (2006). Depletion of CHK1, but not CHK2, induces chromosomal instability and breaks at common fragile sites. Oncogene 25, 4381-4388.

Edmunds, C.E., Simpson, L.J., and Sale, J.E. (2008). PCNA ubiquitination and REV1 define temporally distinct mechanisms for controlling translesion synthesis in the avian cell line DT40. Molecular cell 30, 519-529.

Elvers, I., Johansson, F., Groth, P., Erixon, K., and Helleday, T. (2011). UV stalled replication forks restart by re-priming in human fibroblasts. Nucleic acids research 39, 7049-7057.

Engel, K., Kotlyarov, A., and Gaestel, M. (1998). Leptomycin B-sensitive nuclear export of MAPKAP kinase 2 is regulated by phosphorylation. The EMBO journal 17, 3363-3371.

Engel, K., Plath, K., and Gaestel, M. (1993). The MAP kinase-activated protein kinase 2 contains a proline-rich SH3-binding domain. FEBS letters 336, 143-147.

Engel, K., Schultz, H., Martin, F., Kotlyarov, A., Plath, K., Hahn, M., Heinemann, U., and Gaestel, M. (1995). Constitutive activation of mitogen-activated protein kinase-activated protein kinase 2 by mutation of phosphorylation sites and an A-helix motif. The Journal of biological chemistry 270, 27213-27221.

Espinosa, E., Zamora, P., Feliu, J., and Gonzalez Baron, M. (2003). Classification of anticancer drugs--a new system based on therapeutic targets. Cancer treatment reviews 29, 515-523.

Esposito, G., Godindagger, I., Klein, U., Yaspo, M.L., Cumano, A., and Rajewsky, K. (2000). Disruption of the Rev3l-encoded catalytic subunit of polymerase zeta in mice results in early embryonic lethality. Current biology : CB 10,1221-1224.

Ewald, B., Sampath, D., and Plunkett, W. (2008). Nucleoside analogs: molecular mechanisms signaling cell death. Oncogene 27, 6522-6537. 
Feijoo, C., Hall-Jackson, C., Wu, R., Jenkins, D., Leitch, J., Gilbert, D.M., and Smythe, C. (2001). Activation of mammalian Chk1 during DNA replication arrest: a role for Chk 1 in the intra-S phase checkpoint monitoring replication origin firing. The Journal of cell biology $154,913-923$.

Feng, W., Collingwood, D., Boeck, M.E., Fox, L.A., Alvino, G.M., Fangman, W.L., Raghuraman, M.K., and Brewer, B.J. (2006). Genomic mapping of single-stranded DNA in hydroxyurea-challenged yeasts identifies origins of replication. Nature cell biology 8 , 148-155.

Fernandez-Capetillo, O., Lee, A., Nussenzweig, M., and Nussenzweig, A. (2004). H2AX: the histone guardian of the genome. DNA repair 3, 959-967.

Forment, J.V., Blasius, M., Guerini, I., and Jackson, S.P. (2011). Structure-specific DNA endonuclease Mus81/Eme1 generates DNA damage caused by Chk1 inactivation. PloS one 6 , e23517.

Forsburg, S.L. (2008). The MCM helicase: linking checkpoints to the replication fork. Biochemical Society transactions 36, 114-119.

Freeman, A.K., and Monteiro, A.N. (2010). Phosphatases in the cellular response to DNA damage. Cell communication and signaling : CCS 8, 27.

Friedberg, E.C., Wagner, R., and Radman, M. (2002). Specialized DNA polymerases, cellular survival, and the genesis of mutations. Science 296, 1627-1630.

Fryer, R.A., Barlett, B., Galustian, C., and Dalgleish, A.G. (2011). Mechanisms underlying gemcitabine resistance in pancreatic cancer and sensitisation by the iMiD lenalidomide. Anticancer research 31, 3747-3756.

Gaestel, M. (2006). MAPKAP kinases - MKs - two's company, three's a crowd. Nature reviews Molecular cell biology 7, 120-130.

Gagou, M.E., Zuazua-Villar, P., and Meuth, M. (2010). Enhanced H2AX phosphorylation, DNA replication fork arrest, and cell death in the absence of Chk1. Molecular biology of the cell 21, 739-752.

Galmarini, C.M., Mackey, J.R., and Dumontet, C. (2002). Nucleoside analogues and nucleobases in cancer treatment. The lancet oncology 3, 415-424.

Ge, X.Q., and Blow, J.J. (2010). Chk1 inhibits replication factory activation but allows dormant origin firing in existing factories. The Journal of cell biology 191, 1285-1297.

Ge, X.Q., Jackson, D.A., and Blow, J.J. (2007). Dormant origins licensed by excess Mcm2-7 are required for human cells to survive replicative stress. Genes \& development $21,3331-3341$.

Gohler, T., Sabbioneda, S., Green, C.M., and Lehmann, A.R. (2011). ATR-mediated phosphorylation of DNA polymerase eta is needed for efficient recovery from UV damage. The Journal of cell biology 192, 219-227.

Gu, Y., Rosenblatt, J., and Morgan, D.O. (1992). Cell cycle regulation of CDK2 activity by phosphorylation of Thr160 and Tyr15. The EMBO journal 11, 3995-4005. 
Guay, J., Lambert, H., Gingras-Breton, G., Lavoie, J.N., Huot, J., and Landry, J. (1997). Regulation of actin filament dynamics by p38 map kinase-mediated phosphorylation of heat shock protein 27. Journal of cell science 110 (Pt 3), 357-368.

Guo, C., Tang, T.S., Bienko, M., Parker, J.L., Bielen, A.B., Sonoda, E., Takeda, S., Ulrich, H.D., Dikic, I., and Friedberg, E.C. (2006). Ubiquitin-binding motifs in REV1 protein are required for its role in the tolerance of DNA damage. Molecular and cellular biology 26, 8892-8900.

Guo, Z., Kumagai, A., Wang, S.X., and Dunphy, W.G. (2000). Requirement for Atr in phosphorylation of Chk1 and cell cycle regulation in response to DNA replication blocks and UV-damaged DNA in Xenopus egg extracts. Genes \& development 14, 2745-2756.

Hartlerode, A.J., and Scully, R. (2009). Mechanisms of double-strand break repair in somatic mammalian cells. The Biochemical journal 423, 157-168.

Heffernan, T.P., Simpson, D.A., Frank, A.R., Heinloth, A.N., Paules, R.S., Cordeiro-Stone, M., and Kaufmann, W.K. (2002). An ATR- and Chk1-dependent S checkpoint inhibits replicon initiation following UVC-induced DNA damage. Molecular and cellular biology 22, 8552-8561.

Heffernan, T.P., Unsal-Kacmaz, K., Heinloth, A.N., Simpson, D.A., Paules, R.S., Sancar, A., Cordeiro-Stone, M., and Kaufmann, W.K. (2007). Cdc7-Dbf4 and the human S checkpoint response to UVC. The Journal of biological chemistry 282, 9458-9468.

Herrlich, P., Karin, M., and Weiss, C. (2008). Supreme EnLIGHTenment: damage recognition and signaling in the mammalian UV response. Molecular cell 29, 279-290.

Hochegger, H., Dejsuphong, D., Sonoda, E., Saberi, A., Rajendra, E., Kirk, J., Hunt, T., and Takeda, S. (2007). An essential role for Cdk1 in S phase control is revealed via chemical genetics in vertebrate cells. The Journal of cell biology 178, 257-268.

Hoege, C., Pfander, B., Moldovan, G.L., Pyrowolakis, G., and Jentsch, S. (2002). RAD6dependent DNA repair is linked to modification of PCNA by ubiquitin and SUMO. Nature $419,135-141$.

Hoeijmakers, J.H. (2009). DNA damage, aging, and cancer. The New England journal of medicine $361,1475-1485$.

Hu, J.H., Chen, T., Zhuang, Z.H., Kong, L., Yu, M.C., Liu, Y., Zang, J.W., and Ge, B.X. (2007). Feedback control of MKP-1 expression by p38. Cellular signalling 19, 393-400.

Huang, P., Chubb, S., Hertel, L.W., Grindey, G.B., and Plunkett, W. (1991). Action of 2',2'difluorodeoxycytidine on DNA synthesis. Cancer research 51, 6110-6117.

Hung, S.W., Mody, H.R., and Govindarajan, R. (2012). Overcoming nucleoside analog chemoresistance of pancreatic cancer: a therapeutic challenge. Cancer letters 320, 138149.

Ibarra, A., Schwob, E., and Mendez, J. (2008). Excess MCM proteins protect human cells from replicative stress by licensing backup origins of replication. Proceedings of the National Academy of Sciences of the United States of America 105, 8956-8961.

Ishimi, Y., Komamura-Kohno, Y., Kwon, H.J., Yamada, K., and Nakanishi, M. (2003). Identification of MCM4 as a target of the DNA replication block checkpoint system. The Journal of biological chemistry $278,24644-24650$. 
Jackson, A.L., and Loeb, L.A. (2001). The contribution of endogenous sources of DNA damage to the multiple mutations in cancer. Mutation research 477, 7-21.

Jackson, D.A., and Pombo, A. (1998). Replicon clusters are stable units of chromosome structure: evidence that nuclear organization contributes to the efficient activation and propagation of S phase in human cells. The Journal of cell biology 140, 1285-1295.

Jackson, J.R., Gilmartin, A., Imburgia, C., Winkler, J.D., Marshall, L.A., and Roshak, A. (2000). An indolocarbazole inhibitor of human checkpoint kinase (Chk1) abrogates cell cycle arrest caused by DNA damage. Cancer research 60, 566-572.

Jansen, J.G., Fousteri, M.I., and de Wind, N. (2007). Send in the clamps: control of DNA translesion synthesis in eukaryotes. Molecular cell 28, 522-529.

Johansen, C., Vestergaard, C., Kragballe, K., Kollias, G., Gaestel, M., and Iversen, L. (2009). MK2 regulates the early stages of skin tumor promotion. Carcinogenesis 30, 2100-2108.

Johnson, R.E., Prakash, S., and Prakash, L. (1999). Efficient bypass of a thyminethymine dimer by yeast DNA polymerase, Poleta. Science 283, 1001-1004.

Johnson, R.E., Washington, M.T., Haracska, L., Prakash, S., and Prakash, L. (2000). Eukaryotic polymerases iota and zeta act sequentially to bypass DNA lesions. Nature 406, 1015-1019.

Kai, M., Boddy, M.N., Russell, P., and Wang, T.S. (2005). Replication checkpoint kinase Cds1 regulates Mus81 to preserve genome integrity during replication stress. Genes \& development 19, 919-932.

Kannouche, P.L., Wing, J., and Lehmann, A.R. (2004). Interaction of human DNA polymerase eta with monoubiquitinated PCNA: a possible mechanism for the polymerase switch in response to DNA damage. Molecular cell 14, 491-500.

Kastan, M.B., and Bartek, J. (2004). Cell-cycle checkpoints and cancer. Nature 432, 316323.

Katsuno, Y., Suzuki, A., Sugimura, K., Okumura, K., Zineldeen, D.H., Shimada, M., Niida, H., Mizuno, T., Hanaoka, F., and Nakanishi, M. (2009). Cyclin A-Cdk1 regulates the origin firing program in mammalian cells. Proceedings of the National Academy of Sciences of the United States of America 106, 3184-3189.

Kinner, A., Wu, W., Staudt, C., and Iliakis, G. (2008). Gamma-H2AX in recognition and signaling of DNA double-strand breaks in the context of chromatin. Nucleic acids research $36,5678-5694$.

Koizumi, K., Tanno, S., Nakano, Y., Habiro, A., Izawa, T., Mizukami, Y., Okumura, T., and Kohgo, Y. (2005). Activation of p38 mitogen-activated protein kinase is necessary for gemcitabine-induced cytotoxicity in human pancreatic cancer cells. Anticancer research 25, 3347-3353.

Konerding, D., James, T.L., Trump, E., Soto, A.M., Marky, L.A., and Gmeiner, W.H. (2002). NMR structure of a gemcitabine-substituted model Okazaki fragment. Biochemistry 41, 839-846.

Koren, A. (2007). The role of the DNA damage checkpoint in regulation of translesion DNA synthesis. Mutagenesis 22, 155-160. 
Kotlyarov, A., Neininger, A., Schubert, C., Eckert, R., Birchmeier, C., Volk, H.D., and Gaestel, M. (1999). MAPKAP kinase 2 is essential for LPS-induced TNF-alpha biosynthesis. Nature cell biology 1, 94-97.

Kranz, D., and Dobbelstein, M. (2006). Nongenotoxic p53 activation protects cells against S-phase-specific chemotherapy. Cancer research 66, 10274-10280.

Kranz, D., Dohmesen, C., and Dobbelstein, M. (2008). BRCA1 and Tip60 determine the cellular response to ultraviolet irradiation through distinct pathways. The Journal of cell biology 182, 197-213.

Kumagai, A., Kim, S.M., and Dunphy, W.G. (2004). Claspin and the activated form of ATR-ATRIP collaborate in the activation of Chk1. The Journal of biological chemistry 279, 49599-49608.

Laemmli, U.K. (1970). Cleavage of structural proteins during the assembly of the head of bacteriophage T4. Nature 227, 680-685.

Lehner, M.D., Schwoebel, F., Kotlyarov, A., Leist, M., Gaestel, M., and Hartung, T. (2002). Mitogen-activated protein kinase-activated protein kinase 2-deficient mice show increased susceptibility to Listeria monocytogenes infection. J Immunol 168, 4667-4673.

Li, J., Uchida, T., Todo, T., and Kitagawa, T. (2006). Similarities and differences between cyclobutane pyrimidine dimer photolyase and (6-4) photolyase as revealed by resonance Raman spectroscopy: Electron transfer from the FAD cofactor to ultraviolet-damaged DNA. The Journal of biological chemistry 281, 25551-25559.

Lin, X., and Howell, S.B. (2006). DNA mismatch repair and p53 function are major determinants of the rate of development of cisplatin resistance. Molecular cancer therapeutics $5,1239-1247$.

Lindahl, T. (2004). Molecular biology: ensuring error-free DNA repair. Nature 427, 598.

Lindahl, T., and Barnes, D.E. (2000). Repair of endogenous DNA damage. Cold Spring Harbor symposia on quantitative biology 65, 127-133.

Ling, H., Sayer, J.M., Plosky, B.S., Yagi, H., Boudsocq, F., Woodgate, R., Jerina, D.M., and Yang, W. (2004). Crystal structure of a benzo[a]pyrene diol epoxide adduct in a ternary complex with a DNA polymerase. Proceedings of the National Academy of Sciences of the United States of America 101, 2265-2269.

Liu, P., Barkley, L.R., Day, T., Bi, X., Slater, D.M., Alexandrow, M.G., Nasheuer, H.P., and Vaziri, C. (2006). The Chk1-mediated S-phase checkpoint targets initiation factor Cdc45 via a Cdc25A/Cdk2-independent mechanism. The Journal of biological chemistry 281, 30631-30644.

Liu, Q., Guntuku, S., Cui, X.S., Matsuoka, S., Cortez, D., Tamai, K., Luo, G., CarattiniRivera, S., DeMayo, F., Bradley, A., et al. (2000). Chk1 is an essential kinase that is regulated by Atr and required for the $\mathrm{G}(2) / \mathrm{M}$ DNA damage checkpoint. Genes \& development 14, 1448-1459.

Long, J., Zhang, Y., Yu, X., Yang, J., LeBrun, D.G., Chen, C., Yao, Q., and Li, M. (2011). Overcoming drug resistance in pancreatic cancer. Expert opinion on therapeutic targets $15,817-828$. 
Ma, C.X., Janetka, J.W., and Piwnica-Worms, H. (2011). Death by releasing the breaks: CHK1 inhibitors as cancer therapeutics. Trends in molecular medicine 17, 88-96.

Manke, I.A., Nguyen, A., Lim, D., Stewart, M.Q., Elia, A.E., and Yaffe, M.B. (2005). MAPKAP kinase-2 is a cell cycle checkpoint kinase that regulates the G2/M transition and $S$ phase progression in response to UV irradiation. Molecular cell 17, 37-48.

Masai, H., Taniyama, C., Ogino, K., Matsui, E., Kakusho, N., Matsumoto, S., Kim, J.M., Ishii, A., Tanaka, T., Kobayashi, T., et al. (2006). Phosphorylation of MCM4 by Cdc7 kinase facilitates its interaction with Cdc45 on the chromatin. The Journal of biological chemistry 281, 39249-39261.

Massague, J. (2004). G1 cell-cycle control and cancer. Nature 432, 298-306.

Matsuoka, S., Ballif, B.A., Smogorzewska, A., McDonald, E.R., 3rd, Hurov, K.E., Luo, J., Bakalarski, C.E., Zhao, Z., Solimini, N., Lerenthal, Y., et al. (2007). ATM and ATR substrate analysis reveals extensive protein networks responsive to DNA damage. Science 316, 1160-1166.

Maya-Mendoza, A., Petermann, E., Gillespie, D.A., Caldecott, K.W., and Jackson, D.A. (2007). Chk1 regulates the density of active replication origins during the vertebrate $S$ phase. The EMBO journal 26, 2719-2731.

McGowan, C.H., and Russell, P. (1993). Human Wee1 kinase inhibits cell division by phosphorylating p34cdc2 exclusively on Tyr15. The EMBO journal 12, 75-85.

Medema, R.H., and Macurek, L. (2012). Checkpoint control and cancer. Oncogene 31, 2601-2613.

Medhurst, A.L., Warmerdam, D.O., Akerman, I., Verwayen, E.H., Kanaar, R., Smits, V.A., and Lakin, N.D. (2008). ATR and Rad17 collaborate in modulating Rad9 localisation at sites of DNA damage. Journal of cell science 121, 3933-3940.

Meek, D.W. (2002). p53 Induction: phosphorylation sites cooperate in regulating. Cancer biology \& therapy $1,284-286$.

Meek, D.W., and Knippschild, U. (2003). Posttranslational modification of MDM2. Molecular cancer research : MCR 1, 1017-1026.

Meek, K., Dang, V., and Lees-Miller, S.P. (2008). DNA-PK: the means to justify the ends? Advances in immunology 99, 33-58.

Meng, W., Swenson, L.L., Fitzgibbon, M.J., Hayakawa, K., Ter Haar, E., Behrens, A.E., Fulghum, J.R., and Lippke, J.A. (2002). Structure of mitogen-activated protein kinaseactivated protein (MAPKAP) kinase 2 suggests a bifunctional switch that couples kinase activation with nuclear export. The Journal of biological chemistry 277, 37401-37405.

Miao, H., Seiler, J.A., and Burhans, W.C. (2003). Regulation of cellular and SV40 virus origins of replication by Chk1-dependent intrinsic and UVC radiation-induced checkpoints. The Journal of biological chemistry $278,4295-4304$.

Mini, E., Nobili, S., Caciagli, B., Landini, I., and Mazzei, T. (2006). Cellular pharmacology of gemcitabine. Annals of oncology : official journal of the European Society for Medical Oncology / ESMO 17 Suppl 5, v7-12. 
Moldovan, G.L., Pfander, B., and Jentsch, S. (2007). PCNA, the maestro of the replication fork. Cell 129, 665-679.

Murga, M., Bunting, S., Montana, M.F., Soria, R., Mulero, F., Canamero, M., Lee, Y., McKinnon, P.J., Nussenzweig, A., and Fernandez-Capetillo, O. (2009). A mouse model of ATR-Seckel shows embryonic replicative stress and accelerated aging. Nature genetics 41, 891-898.

Myung, K., and Kolodner, R.D. (2002). Suppression of genome instability by redundant Sphase checkpoint pathways in Saccharomyces cerevisiae. Proceedings of the National Academy of Sciences of the United States of America 99, 4500-4507.

Nelson, J.R., Lawrence, C.W., and Hinkle, D.C. (1996). Deoxycytidyl transferase activity of yeast REV1 protein. Nature 382, 729-731.

Norbury, C., Blow, J., and Nurse, P. (1991). Regulatory phosphorylation of the p34cdc2 protein kinase in vertebrates. The EMBO journal 10, 3321-3329.

O'Connell, M.J., Raleigh, J.M., Verkade, H.M., and Nurse, P. (1997). Chk1 is a wee1 kinase in the G2 DNA damage checkpoint inhibiting cdc2 by Y15 phosphorylation. The EMBO journal 16, 545-554.

Petermann, E., and Helleday, T. (2010). Pathways of mammalian replication fork restart. Nature reviews Molecular cell biology 11, 683-687.

Petermann, E., Maya-Mendoza, A., Zachos, G., Gillespie, D.A., Jackson, D.A., and Caldecott, K.W. (2006). Chk1 requirement for high global rates of replication fork progression during normal vertebrate $S$ phase. Molecular and cellular biology 26, 33193326.

Petermann, E., Orta, M.L., Issaeva, N., Schultz, N., and Helleday, T. (2010a). Hydroxyurea-stalled replication forks become progressively inactivated and require two different RAD51-mediated pathways for restart and repair. Molecular cell 37, 492-502.

Petermann, E., Woodcock, M., and Helleday, T. (2010b). Chk1 promotes replication fork progression by controlling replication initiation. Proceedings of the National Academy of Sciences of the United States of America 107, 16090-16095.

Prakash, S., Johnson, R.E., and Prakash, L. (2005). Eukaryotic translesion synthesis DNA polymerases: specificity of structure and function. Annual review of biochemistry 74, 317-353.

Raleigh, J.M., and O'Connell, M.J. (2000). The G(2) DNA damage checkpoint targets both Wee1 and Cdc25. Journal of cell science 113 ( Pt 10), 1727-1736.

Raman, M., Earnest, S., Zhang, K., Zhao, Y., and Cobb, M.H. (2007). TAO kinases mediate activation of p38 in response to DNA damage. The EMBO journal 26, 2005-2014.

Regairaz, M., Zhang, Y.W., Fu, H., Agama, K.K., Tata, N., Agrawal, S., Aladjem, M.I., and Pommier, Y. (2011). Mus81-mediated DNA cleavage resolves replication forks stalled by topoisomerase I-DNA complexes. The Journal of cell biology 195, 739-749.

Reinhardt, H.C., Aslanian, A.S., Lees, J.A., and Yaffe, M.B. (2007). p53-deficient cells rely on ATM- and ATR-mediated checkpoint signaling through the p38MAPK/MK2 pathway for survival after DNA damage. Cancer cell 11, 175-189. 
Reinhardt, H.C., Hasskamp, P., Schmedding, I., Morandell, S., van Vugt, M.A., Wang, X., Linding, R., Ong, S.E., Weaver, D., Carr, S.A., et al. (2010). DNA damage activates a spatially distinct late cytoplasmic cell-cycle checkpoint network controlled by MK2mediated RNA stabilization. Molecular cell 40, 34-49.

Renart, J., Reiser, J., and Stark, G.R. (1979). Transfer of proteins from gels to diazobenzyloxymethyl-paper and detection with antisera: a method for studying antibody specificity and antigen structure. Proceedings of the National Academy of Sciences of the United States of America 76, 3116-3120.

Rogakou, E.P., Boon, C., Redon, C., and Bonner, W.M. (1999). Megabase chromatin domains involved in DNA double-strand breaks in vivo. The Journal of cell biology 146, 905-916.

Rogakou, E.P., Pilch, D.R., Orr, A.H., Ivanova, V.S., and Bonner, W.M. (1998). DNA double-stranded breaks induce histone H2AX phosphorylation on serine 139. The Journal of biological chemistry $273,5858-5868$.

Rogalla, T., Ehrnsperger, M., Preville, X., Kotlyarov, A., Lutsch, G., Ducasse, C., Paul, C., Wieske, M., Arrigo, A.P., Buchner, J., et al. (1999). Regulation of Hsp27 oligomerization, chaperone function, and protective activity against oxidative stress/tumor necrosis factor alpha by phosphorylation. The Journal of biological chemistry 274, 18947-18956.

Ronkina, N., Kotlyarov, A., Dittrich-Breiholz, O., Kracht, M., Hitti, E., Milarski, K., Askew, R., Marusic, S., Lin, L.L., Gaestel, M., et al. (2007). The mitogen-activated protein kinase (MAPK)-activated protein kinases MK2 and MK3 cooperate in stimulation of tumor necrosis factor biosynthesis and stabilization of p38 MAPK. Molecular and cellular biology 27, 170-181.

Sale, J.E., Lehmann, A.R., and Woodgate, R. (2012). Y-family DNA polymerases and their role in tolerance of cellular DNA damage. Nature reviews Molecular cell biology 13, 141-152.

Sancar, A., Lindsey-Boltz, L.A., Unsal-Kacmaz, K., and Linn, S. (2004). Molecular mechanisms of mammalian DNA repair and the DNA damage checkpoints. Annual review of biochemistry $73,39-85$.

Sanger, F., Nicklen, S., and Coulson, A.R. (1977). DNA sequencing with chainterminating inhibitors. Proceedings of the National Academy of Sciences of the United States of America 74, 5463-5467.

Sarcevic, B., Mawson, A., Baker, R.T., and Sutherland, R.L. (2002). Regulation of the ubiquitin-conjugating enzyme hHR6A by CDK-mediated phosphorylation. The EMBO journal 21, 2009-2018.

Scorah, J., Dong, M.Q., Yates, J.R., 3rd, Scott, M., Gillespie, D., and McGowan, C.H. (2008). A conserved proliferating cell nuclear antigen-interacting protein sequence in Chk1 is required for checkpoint function. The Journal of biological chemistry 283, 1725017259.

Segurado, M., and Tercero, J.A. (2009). The S-phase checkpoint: targeting the replication fork. Biology of the cell / under the auspices of the European Cell Biology Organization 101, 617-627.

Shechter, D., Costanzo, V., and Gautier, J. (2004). ATR and ATM regulate the timing of DNA replication origin firing. Nature cell biology 6, 648-655. 
Shiotani, B., and Zou, L. (2009). Single-stranded DNA orchestrates an ATM-to-ATR switch at DNA breaks. Molecular cell 33, 547-558.

Sorensen, C.S., Hansen, L.T., Dziegielewski, J., Syljuasen, R.G., Lundin, C., Bartek, J., and Helleday, T. (2005). The cell-cycle checkpoint kinase Chk1 is required for mammalian homologous recombination repair. Nature cell biology 7, 195-201.

Sorensen, C.S., and Syljuasen, R.G. (2012). Safeguarding genome integrity: the checkpoint kinases ATR, CHK1 and WEE1 restrain CDK activity during normal DNA replication. Nucleic acids research 40, 477-486.

Speroni, J., Federico, M.B., Mansilla, S.F., Soria, G., and Gottifredi, V. (2012). Kinaseindependent function of checkpoint kinase 1 (Chk1) in the replication of damaged DNA. Proceedings of the National Academy of Sciences of the United States of America 109, 7344-7349.

Stelter, P., and Ulrich, H.D. (2003). Control of spontaneous and damage-induced mutagenesis by SUMO and ubiquitin conjugation. Nature 425, 188-191.

Stiff, T., O'Driscoll, M., Rief, N., Iwabuchi, K., Lobrich, M., and Jeggo, P.A. (2004). ATM and DNA-PK function redundantly to phosphorylate $\mathrm{H} 2 \mathrm{AX}$ after exposure to ionizing radiation. Cancer research 64, 2390-2396.

Stokoe, D., Campbell, D.G., Nakielny, S., Hidaka, H., Leevers, S.J., Marshall, C., and Cohen, P. (1992a). MAPKAP kinase-2; a novel protein kinase activated by mitogenactivated protein kinase. The EMBO journal 11, 3985-3994.

Stokoe, D., Caudwell, B., Cohen, P.T., and Cohen, P. (1993). The substrate specificity and structure of mitogen-activated protein (MAP) kinase-activated protein kinase-2. The Biochemical journal 296 ( Pt 3), 843-849.

Stokoe, D., Engel, K., Campbell, D.G., Cohen, P., and Gaestel, M. (1992b). Identification of MAPKAP kinase 2 as a major enzyme responsible for the phosphorylation of the small mammalian heat shock proteins. FEBS letters 313, 307-313.

Stucki, M., and Jackson, S.P. (2006). gammaH2AX and MDC1: anchoring the DNAdamage-response machinery to broken chromosomes. DNA repair 5, 534-543.

Syljuasen, R.G., Sorensen, C.S., Hansen, L.T., Fugger, K., Lundin, C., Johansson, F., Helleday, T., Sehested, M., Lukas, J., and Bartek, J. (2005). Inhibition of human Chk1 causes increased initiation of DNA replication, phosphorylation of ATR targets, and DNA breakage. Molecular and cellular biology 25, 3553-3562.

Tominaga, Y., Li, C., Wang, R.H., and Deng, C.X. (2006). Murine Wee1 plays a critical role in cell cycle regulation and pre-implantation stages of embryonic development. International journal of biological sciences 2, 161-170.

Towbin, H., Staehelin, T., and Gordon, J. (1979). Electrophoretic transfer of proteins from polyacrylamide gels to nitrocellulose sheets: procedure and some applications. Proceedings of the National Academy of Sciences of the United States of America 76, 4350-4354.

Vassilev, L.T., Vu, B.T., Graves, B., Carvajal, D., Podlaski, F., Filipovic, Z., Kong, N., Kammlott, U., Lukacs, C., Klein, C., et al. (2004). In vivo activation of the p53 pathway by small-molecule antagonists of MDM2. Science 303, 844-848. 
Vertii, A., Hakim, C., Kotlyarov, A., and Gaestel, M. (2006). Analysis of properties of small heat shock protein Hsp25 in MAPK-activated protein kinase 2 (MK2)-deficient cells: MK2dependent insolubilization of Hsp25 oligomers correlates with susceptibility to stress. The Journal of biological chemistry 281, 26966-26975.

Vos, M.J., Kanon, B., and Kampinga, H.H. (2009). HSPB7 is a SC35 speckle resident small heat shock protein. Biochimica et biophysica acta 1793, 1343-1353.

Wagner, J.M., and Karnitz, L.M. (2009). Cisplatin-induced DNA damage activates replication checkpoint signaling components that differentially affect tumor cell survival. Molecular pharmacology 76, 208-214.

Walter, J., and Newport, J. (2000). Initiation of eukaryotic DNA replication: origin unwinding and sequential chromatin association of Cdc45, RPA, and DNA polymerase alpha. Molecular cell 5, 617-627.

Wang, H., Wang, M., Bocker, W., and lliakis, G. (2005). Complex H2AX phosphorylation patterns by multiple kinases including ATM and DNA-PK in human cells exposed to ionizing radiation and treated with kinase inhibitors. Journal of cellular physiology 202, 492-502.

Ward, I.M., Minn, K., and Chen, J. (2004). UV-induced ataxia-telangiectasia-mutated and Rad3-related (ATR) activation requires replication stress. The Journal of biological chemistry $279,9677-9680$.

Watanabe, N., Broome, M., and Hunter, T. (1995). Regulation of the human WEE1Hu CDK tyrosine 15-kinase during the cell cycle. The EMBO journal 14, 1878-1891.

Waters, L.S., Minesinger, B.K., Wiltrout, M.E., D'Souza, S., Woodruff, R.V., and Walker, G.C. (2009). Eukaryotic translesion polymerases and their roles and regulation in DNA damage tolerance. Microbiology and molecular biology reviews : MMBR 73, 134-154.

Winzen, R., Kracht, M., Ritter, B., Wilhelm, A., Chen, C.Y., Shyu, A.B., Muller, M., Gaestel, M., Resch, K., and Holtmann, H. (1999). The p38 MAP kinase pathway signals for cytokine-induced mRNA stabilization via MAP kinase-activated protein kinase 2 and an AU-rich region-targeted mechanism. The EMBO journal 18, 4969-4980.

Woodward, A.M., Gohler, T., Luciani, M.G., Oehlmann, M., Ge, X., Gartner, A., Jackson, D.A., and Blow, J.J. (2006). Excess Mcm2-7 license dormant origins of replication that can be used under conditions of replicative stress. The Journal of cell biology 173, 673683.

Xiao, Z., Xue, J., Sowin, T.J., Rosenberg, S.H., and Zhang, H. (2005). A novel mechanism of checkpoint abrogation conferred by Chk1 downregulation. Oncogene 24, 1403-1411.

Xiao, Z., Xue, J., Sowin, T.J., and Zhang, H. (2006). Differential roles of checkpoint kinase 1 , checkpoint kinase 2, and mitogen-activated protein kinase-activated protein kinase 2 in mediating DNA damage-induced cell cycle arrest: implications for cancer therapy. Molecular cancer therapeutics 5, 1935-1943.

Yang, X.H., Shiotani, B., Classon, M., and Zou, L. (2008). Chk1 and Claspin potentiate PCNA ubiquitination. Genes \& development 22, 1147-1152.

Yang, X.H., and Zou, L. (2009). Dual functions of DNA replication forks in checkpoint signaling and PCNA ubiquitination. Cell Cycle 8, 191-194. 
Yoshizawa-Sugata, N., Ishii, A., Taniyama, C., Matsui, E., Arai, K., and Masai, H. (2005). A second human Dbf4/ASK-related protein, Drf1/ASKL1, is required for efficient progression of $S$ and M phases. The Journal of biological chemistry 280, 13062-13070.

Zachos, G., Rainey, M.D., and Gillespie, D.A. (2003). Chk1-deficient tumour cells are viable but exhibit multiple checkpoint and survival defects. The EMBO journal 22, 713723.

Zakowski, V., Keramas, G., Kilian, K., Rapp, U.R., and Ludwig, S. (2004). Mitogenactivated 3p kinase is active in the nucleus. Experimental cell research 299, 101-109.

Zenvirt, S., Kravchenko-Balasha, N., and Levitzki, A. (2010). Status of p53 in human cancer cells does not predict efficacy of CHK1 kinase inhibitors combined with chemotherapeutic agents. Oncogene 29, 6149-6159.

Zhao, H., and Piwnica-Worms, H. (2001). ATR-mediated checkpoint pathways regulate phosphorylation and activation of human Chk1. Molecular and cellular biology 21, 41294139.

Zhao, H., Watkins, J.L., and Piwnica-Worms, H. (2002). Disruption of the checkpoint kinase $1 /$ cell division cycle 25A pathway abrogates ionizing radiation-induced $S$ and G2 checkpoints. Proceedings of the National Academy of Sciences of the United States of America 99, 14795-14800.

Zhou, B.B., and Elledge, S.J. (2000). The DNA damage response: putting checkpoints in perspective. Nature 408, 433-439.

Zou, L., Liu, D., and Elledge, S.J. (2003). Replication protein A-mediated recruitment and activation of Rad17 complexes. Proceedings of the National Academy of Sciences of the United States of America 100, 13827-13832. 
117

APPENDIX

Vector maps of pcDNA3 and pcDNA3-Myc-MK2 WT:
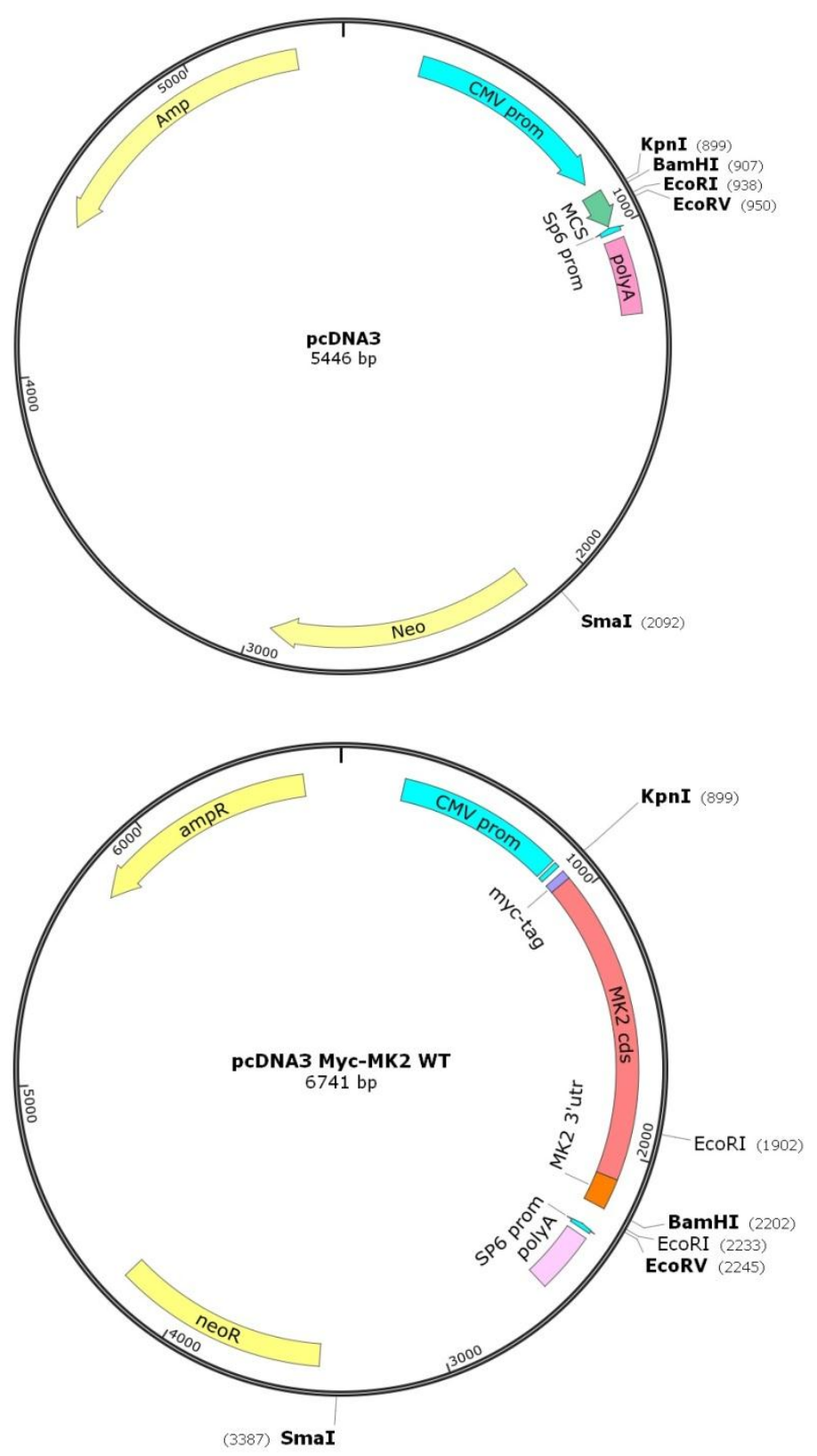


\section{Vector map and MCS of pIRESneo:}

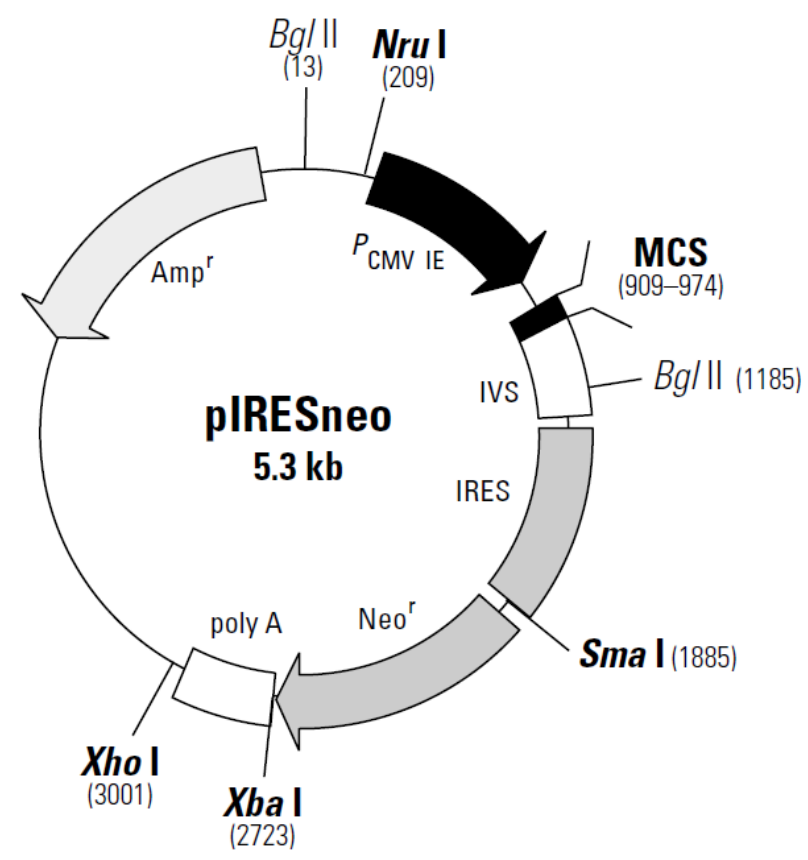

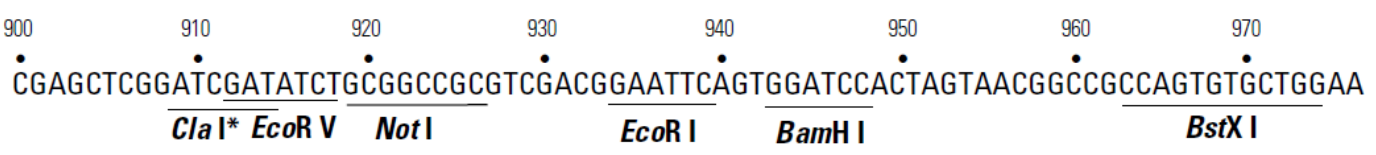

(C) 1999, CLONTECH Laboratories, Inc.

\section{Alignment of human (hs) and mouse $(\mathrm{mm})$ MK2 protein sequence:}

\begin{tabular}{|c|c|c|}
\hline $\begin{array}{l}\text { hs } \\
106\end{array}$ & 47 & $\begin{array}{l}\text { HVKSGLQIKKNAI I DDYKVTSQVLGLGINGKVLQI FNKRTQEKFALKMLQDCPKARREVE } \\
\text { HVKSGLQI+KNAI DDYKVTSOVLGLGINGKVL+IF+KRTQ+KFALKMLQDCPKARREVE }\end{array}$ \\
\hline & 33 & HVKSGLQIRKNAITDDYKVTSQVLGLGINGKVLRIFDKRTQQKFALKMLQDCPKARREVE \\
\hline & 107 & $\begin{array}{l}\text { LHWRASQCPHIVRIVDVYENLYAGRKCLLIVMECLDGGELFSRIQDRGDQAFTEREASEI } \\
\text { LHWRASQCPHIV IVDVYENLYAGRKCLLIVMECLDGGELFSRIQDRGDQAFTEREASEI }\end{array}$ \\
\hline & 93 & LHWRASQCPHIVHIVDVYENLYAGRKCLLIVMECLDGGELFSRIQDRGDQAFTEREASEI \\
\hline & 167 & $\begin{array}{l}\text { MKS IGEAIQYLHSINIAHRDVKPENLLYTSKRPNAILKLTDFGFAKETTSHNSLTTPCYT } \\
\text { MKSIGEAIQYLHSINIAHRDVKPENLLYTSKRPNAILKLTDFGFAKETTSHNSLTTPCYT }\end{array}$ \\
\hline 12 & 153 & MKS IGEAIQYLHSINIAHRDVKPENLLYTSKRPNAILKLTDFGFAKETTSHNSLTTPCYT \\
\hline & 227 & $\begin{array}{l}\text { PYYVAPEVLGPEKYDKSCDMWSLGVIMYILLCGYPPFYSNHGLAIS PGMKTRIRMGQYEF } \\
\text { PYYVAPEVLGPEKYDKSCDMWSLGVIMYILLCGYPPFYSNHGLAIS PGMKTRIRMGQYEF }\end{array}$ \\
\hline th & 213 & PYYVAPEVLGPEKYDKSCDMWSLGVIMYILLCGYPPFYSNHGLAISPGMKTRIRMGQYEF \\
\hline & 287 & $\begin{array}{l}\text { PNPEWSEVSEEVKMLIRNLLKTEPTQRMT ITEFMNHPWIMQSTKVPQTPLHTSRVLKEDK } \\
\text { PNPEWSEVSEEVKMLIRNLLKTEPTQRMTITEFMNHPWIMQSTKVPQTPLHTSRVLKEDK }\end{array}$ \\
\hline 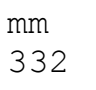 & 273 & PNPEWSEVSEEVKMLIRNLLKTEPTQRMT ITEFMNHPWIMQSTKVPQTPLHTSRVLKEDK \\
\hline & 34 & $\begin{array}{ll}\text { ERWEDVKEEMTSALATMRVDYEQIKIKKIEDASNPLLLKRRKKARALEAAALAH } & 400 \\
\text { ERWEDVKEEMTSALATMRVDYEQIKIKKIEDASNPLLLKRRKKARA+E AALAH } & \end{array}$ \\
\hline & 3 & MRVDYEQIKIKKIEDASNPLLLKRRKKARAVEDAALAH \\
\hline
\end{tabular}

